\title{
A Theoretical and Experimental Investigation on Ejector Acoustics and Ejector Silencer Design
}

\author{
by \\ Gerard Desmarais, B.Eng.

\begin{abstract}
A thesis submitted to the
Faculty of Graduate and Postdoctoral Affairs

in partial fulfillment of the requirements for the degree of
\end{abstract}

Master's of Applied Science in Aerospace Engineering

Ottawa-Carleton Institute for Mechanical and Aerospace Engineering

Department of Mechanical and Aerospace Engineering

Carleton University

Ottawa, Ontario

September, 2018 
(C) Copyright

Gerard Desmarais, 2018 
The undersigned hereby recommends to the

Faculty of Graduate and Postdoctoral Affairs acceptance of the thesis

\title{
A Theoretical and Experimental Investigation on Ejector Acoustics and Ejector Silencer Design
}

\author{
submitted by Gerard Desmarais, B.Eng. \\ in partial fulfillment of the requirements for the degree of
}

Master's of Applied Science in Aerospace Engineering

Professor Joana Rocha, Thesis Supervisor

Professor Ronald E. Miller, Chair,

Department of Mechanical and Aerospace Engineering

Ottawa-Carleton Institute for Mechanical and Aerospace Engineering

Department of Mechanical and Aerospace Engineering

Carleton University

September, 2018 


\section{Abstract}

Ejectors are common devices used across many industries, however, they are often plagued by the presence of low frequency pressure pulsations causing both broadband and tonal noise. This thesis presents a theoretical and experimental investigation into the acoustics of an ejector-silencer combination with the emphasis placed on silencer design. More specifically, the noise generated by the jet, the diffuser and natural modes is explained and estimated. The noise generation mechanisms of impingement tones and edgetones are presented but accurate predictions are found to be hard to make. An outline of the experimental facility is followed by the mechanical, acoustical and aerodynamic design details of the approximately 70:1 ejector scale model. A detailed analysis on the primary nozzle control and calibration is presented before beginning an aerodynamic and acoustic characterization of both the ejector and the wind tunnel facility. From experimentation, it is found that the placement of a perforated cone in front of the solid cone is beneficial in reducing the noise generated without overly affecting the entrainment ratio. Other configurations tested tend not to be as acoustically effective or to decrease the entrainment ratio below an acceptable level. Experiments prove that the low frequency noise generated by the ejector is mainly caused by natural mode excitation. 


\section{Acknowledgements}

First and foremost I would like to thank my family and loved ones who were understanding of the long hours worked on this project and who were always by my side sympathizing with the hardships I faced. I am grateful to have a father who always taught me to dream big and act bigger, which has helped me get to where I am today.

I would like to thank my research supervisor Professor Joana Rocha for presenting me with the opportunity to work on this project as well as for continuous consultation and support over the past two years. Without you this project would have been impossible. The kind and generous contributions of the team at MDS Aero helped move this project along and I am thankful for your support as well as for always making me feel like part of the team.

To my past and present colleagues working under under the supervision of Professor Rocha, your patience and friendship proved invaluable. The input of John O'keefe for aerodynamics portion and Frank Giardino for essentially everything was of great value and I thank you both for that. I would like to express my utmost appreciation to Alex Proctor, Kevin Sangster and Ian Lloy for their seemingly limitless patience and for welcoming me into their shop for many weeks of manufacturing. Without you this research would be nothing more than an idea and a few computer drawings. 


\section{Table of Contents}

Abstract iv

Acknowledgements $\quad$ v

Table of Contents vi

List of Figures $\quad$ xii

List of Tables $\quad \mathrm{xx}$

Nomenclature xxii

\begin{tabular}{lll}
\hline 1 & Introduction & 1
\end{tabular}

1.1 Ejector Description and Basic Principle of Operation . . . . . . . . 2

1.2 Motivation and Problem Definition . . . . . . . . . . . . 4

1.3 Research Objectives and Methodology $\ldots \ldots \ldots \ldots$

1.4 Scope and Limitations of Work $\ldots \ldots \ldots \ldots \ldots$

$1.5 \quad$ A Note on Units $\ldots \ldots \ldots \ldots$

\begin{tabular}{lll}
\hline 2 & Literature Review & 9
\end{tabular}

$2.1 \quad$ General Reports $\ldots \ldots \ldots \ldots \ldots$

2.2 Ejector Noise In the Aerospace Industry $\ldots \ldots \ldots \ldots \ldots$

$2.2 .1 \quad$ Experimental Work . . . . . . . . . . . . . . . . . . 11 
2.2 .2 Computational Work $\ldots \ldots \ldots \ldots \ldots$

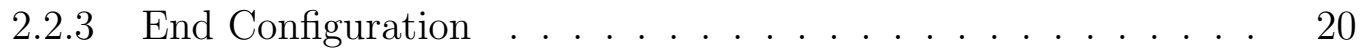

2.3 Closing Remarks $\ldots \ldots \ldots \ldots \ldots$

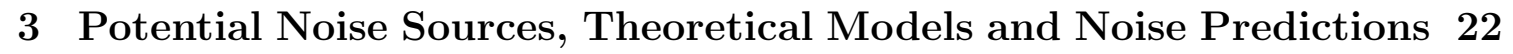

3.1 Jet Noise . . . . . . . . . . . . . . . . . . . . . . . . . . . . . . . . . . . . . 23

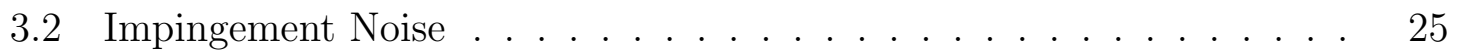

3.3 Edgetone Noise $\ldots \ldots \ldots \ldots$

3.4 Diffuser Noise $\ldots \ldots \ldots \ldots$

$3.5 \quad$ Acoustic Modes . . . . . . . . . . . . . . . . . . . . . . . . . 33

$3.5 .1 \quad$ Acoustic Mode Prediction for the Secondary Flow Chamber . 38

3.5.2 Acoustic Mode Prediction for the Outlet Chamber. . . . . . . 39

$3.5 .3 \quad$ Acoustic Mode Prediction for the Throat . . . . . . . . . . . 40

3.5 .4 Schroeder Frequency . . . . . . . . . . . . . . . . . . 42

$3.5 .5 \quad$ Acoustic Mode Prediction for the Entire Ejector . . . . . . . . 43

3.5 .6 Prediction of the Jet Preferred Frequency. . . . . . . . . . 43

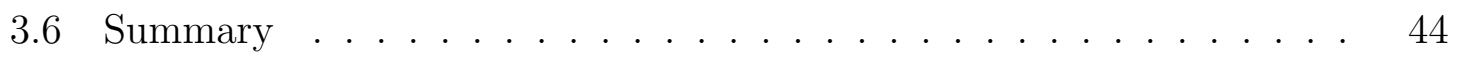

\begin{tabular}{|ll|}
4 Experimental Setup & 45 \\
\hline
\end{tabular}

$4.1 \quad$ Scale Model . . . . . . . . . . . . . . . . . . . . . . . . . . . . . . . 45

4.2 Anechoic Chamber . . . . . . . . . . . . . . . . . . . . 48

$4.3 \quad$ Pratt and Whitney High Speed Wind Tunnel. . . . . . . . . . . . 53

4.4 Facility Layout $\ldots \ldots \ldots \ldots$

4.5 HSWT Velocity Control System . . . . . . . . . . . . . . . 57

$4.5 .1 \quad$ Introduction and Basic Operation Principle $\ldots \ldots \ldots$

4.5 .2 Pressure Measurements for Control . . . . . . . . . . . . 60

4.5 .3 Mach Number Calibration . . . . . . . . . . . . . . . 61 
4.5 .4 Potential Calibration Issues $\ldots \ldots \ldots \ldots$

4.5 .5 PID Control . . . . . . . . . . . . . . . . . . . . 63

4.5.6 Modifications by Hall and Giardino for Improved Operation . 63

4.6 Instrumentation . . . . . . . . . . . . . . . . . . . . . . . . . . . . . . 64

$4.6 .1 \quad$ Pressure Measurement System . . . . . . . . . . . . . . . 66

4.6 .2 Temperature Measurement System … . . . . . . . . . 68

4.6 .3 Microphone Measurement System . . . . . . . . . . . . . . . . 68

4.7 Measurement Uncertainty $\ldots \ldots \ldots \ldots \ldots$

\begin{tabular}{lll}
\hline 5 & Scale Model Design & 76
\end{tabular}

5.1 Scale Factor Rationale . . . . . . . . . . . . . . . . . 76

5.2 Material Selection . . . . . . . . . . . . . . . . . . . . . 79

$5.3 \quad$ Modular Design $\ldots \ldots \ldots \ldots$

$5.3 .1 \quad$ Primary Nozzle $\ldots \ldots \ldots \ldots$

5.3 .2 Throat . . . . . . . . . . . . . . . . . . . 80

5.3 .3 Diffuser $\ldots \ldots \ldots \ldots \ldots \ldots$. . . . . . . . . . . 81

5.3 .4 Perforated Cylinder . . . . . . . . . . . . . . . . . 82

5.3 .5 Cone . . . . . . . . . . . . . . . . . . . . . 82

5.3 .6 Base $\ldots \ldots \ldots \ldots \ldots$

5.4 Primary Nozzle Detailed Mechanical Design . . . . . . . . . . . . 84

$5.4 .1 \quad$ Design Overview . . . . . . . . . . . . . . . . . . 84

5.4 .2 Primary Nozzle Stress $\ldots \ldots \ldots \ldots$

5.5 Scale Model General Structural Considerations . . . . . . . . . . . . 86

5.6 Acoustic Considerations . . . . . . . . . . . . . . . 87

5.7 Scale Model Aerodynamic Design . . . . . . . . . . . . . . . . . 90

5.8 Primary Nozzle Aerodynamic Design $\ldots \ldots \ldots \ldots$ 
5.8 .1 Flow Straightening . . . . . . . . . . . . . . . . . 91

$5.8 .2 \quad$ Primary Nozzle Pressure Ratio . . . . . . . . . . . . . . . . . 97

5.9 Inlet and Outlet Perforated Plate Selection . . . . . . . . . . . . . . . 100

\begin{tabular}{|lll|}
\hline 6 & Jet Velocity Control and Calibration & 101
\end{tabular}

6.1 Data Analysis Procedure . . . . . . . . . . . . . . . . . . . . . . . 101

6.2 Baseline Performance . . . . . . . . . . . . . . . . . . . . . . 103

6.3 Deadband Compensator Off . . . . . . . . . . . . . . . . . . . 104

6.4 Deadband Compensator Variations . . . . . . . . . . . . . . . . 106

6.5 Valve Voltage Analysis . . . . . . . . . . . . . . . . . . . . . 108

6.6 Varying Valve Supply Pressure . . . . . . . . . . . . . . . . . . . . . . 110

6.7 PID Tuning . . . . . . . . . . . . . . . . . . . . . . . . . 111

6.7 .1 "Large" Gain Values . . . . . . . . . . . . . . . . . . . . . . . 112

6.7 .2 "Small" Gain Values . . . . . . . . . . . . . . . . . . . . . . . 114

6.8 Non-Linear Proportional Gain . . . . . . . . . . . . . . . . . . . . 116

6.9 Mach Undershoot . . . . . . . . . . . . . . . . . . . . . . . . . . . 121

6.10 Primary Nozzle Calibration . . . . . . . . . . . . . . . . 124

6.10 .1 Total Pressure Calibration . . . . . . . . . . . . . . . . . . . . 124

6.10 .2 Static Pressure Calibration . . . . . . . . . . . . . . . . . . . . 124

6.10 .3 Mach Verification . . . . . . . . . . . . . . . . 125

6.11 Summary . . . . . . . . . . . . . . . . . 126

\begin{tabular}{|ll|}
7 & Results I: Facility and Model Characterization \\
\hline
\end{tabular}

7.1 Baseline Microphone Performance . . . . . . . . . . . . . . . . . . . . 129

7.1 .1 Microphone Data Analysis Procedure . . . . . . . . . . . . . . 129

7.1 .2 Microphone Calibration Verification . . . . . . . . . . . . . . . 130

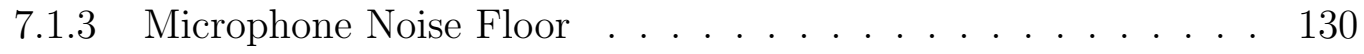


7.2 Background Acoustic Characterization . . . . . . . . . . . . . 132

7.2 .1 Heater Noise $\ldots . . \ldots \ldots$

$7.2 .2 \quad$ Air Dryer Noise . . . . . . . . . . . . . . . . . . . . . . . 132

$7.2 .3 \quad$ Silencer Transmission Noise $\ldots \ldots$. . . . . . . . . . . 134

7.2 .4 Noise at the Valve . . . . . . . . . . . . . . . . 135

$7.2 .5 \quad$ Flexible Air Hose Resonance . . . . . . . . . . . . . . . . 136

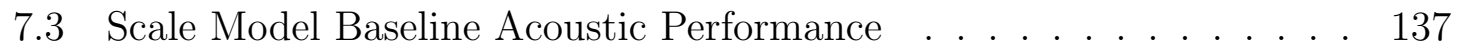

$7.3 .1 \quad$ Modal Decomposition . . . . . . . . . . . . . . . . . . . . 142

$7.4 \quad$ Scale Model Baseline Aerodynamic Performance . . . . . . . . . . . . 146

7.4 .1 Temperature Trend . . . . . . . . . . . . . . . . . 150

8 Results II: Silencer Modifications 151

$8.1 \quad$ Cone Surface Roughness $\ldots \ldots \ldots$

8.2 Closed Cone Rear . . . . . . . . . . . . . . . . . . . . . . . . . 155

$8.3 \quad$ Fin Cone . . . . . . . . . . . . . . . . . . . . . . . . . . . 158

8.4 Closed Bottom Cone . . . . . . . . . . . . . . . . . . . . . 160

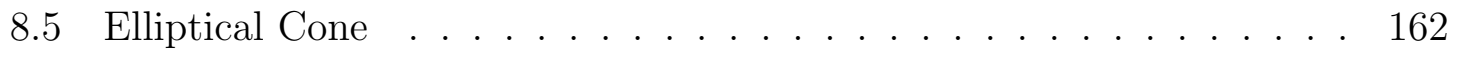

8.6 Perforated Cones . . . . . . . . . . . . . . . . . . . . . . . . 164

8.6 .1 Perforated Cone Replacing The Solid Cone . . . . . . . . . . . 164

8.6 .2 Perforated Cone In Front of Solid Cone . . . . . . . . . . . 166

8.6 .3 Reverse Perforated Cone . . . . . . . . . . . . . . . . . 170

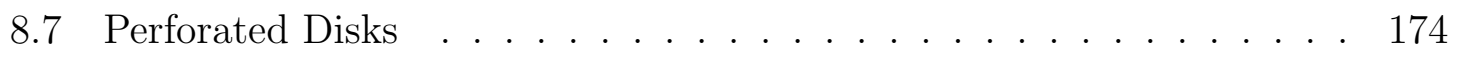

$8.7 .133 \%$ OAR and $58 \%$ OAR $\ldots \ldots \ldots \ldots \ldots$

$8.7 .2 \quad 3$ Perforated Disks $\ldots \ldots \ldots \ldots$. . . . . . . . . . . 177

8.8 Diffuser Modifications $\ldots \ldots \ldots \ldots \ldots$

8.8 .1 Screen at the Diffuser Inlet. . . . . . . . . . . . . . . . . 179 


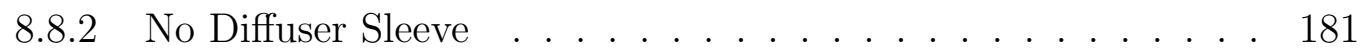

8.9 Natural Mode Elimination . . . . . . . . . . . . . . . . . . . . . 181

8.9 .1 Outlet Flow Chamber Rounded Corners . . . . . . . . . . . . 182

$8.9 .2 \quad$ Scale Model Lined With Acoustic Foam . . . . . . . . . . . . 183

9 Contributions, Conclusions, Recommendations, Lessons Learnt and

$\begin{array}{ll}\text { Future Work } & 188\end{array}$

9.1 Contributions . . . . . . . . . . . . . . . . . . . . . . . 188

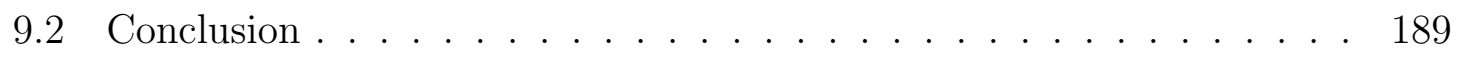

9.3 Recommendations . . . . . . . . . . . . . . . . . . . . . . . . . . 190

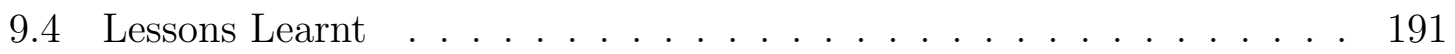

9.5 Future Work . . . . . . . . . . . . . . . . . . . . . . . . . . . . . . . 191

\begin{tabular}{ll}
\hline References & 193
\end{tabular}

\begin{tabular}{|ll}
\hline Appendix A Calibration Curves & 207
\end{tabular}

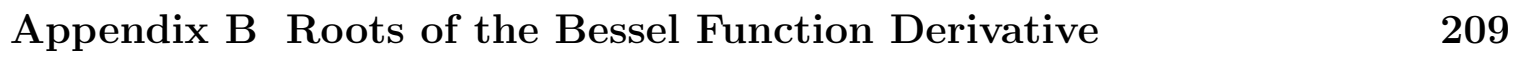

\begin{tabular}{|lll}
\hline Appendix C Noise Measured at the Valve for All Mach Numbers 210 & 210
\end{tabular} 


\section{List of Figures}

$1.1 \quad$ A large steam ejector (left, from [15]) and a mini eductor (right, from $[16]$. . . . . . . . . . . . . . . . . . . . 2

1.2 An ejector schematic outlining the major components . . . . . . . . . 2

1.3 An ejector schematic showing the baseline silencer added aft of the diffuser . . . . . . . . . . . . . . . . . . . . . 6

$2.1 \quad$ A schematic of the test setup used by Middleton, adapted from [19] . 12

2.2 The Schlieren photos taken by reference [31] of a resonant (left) and stable (right) ejector flow, adapted from [31] . . . . . . . . . . . . . . 13

2.3 A Schlieren photograph taken by reference [20] of a resonant ejector flow highlighting the longitudinal and transverse oscillations present, adapted from [20] . . . . . . . . . . . . . . . . . . . . . 15

2.4 The test setup used by [32] (left), with the two screens tested providing good noise suppression (center), and the resulting drop in overall sound pressure level for the baseline (bsln), 16 mesh screen (16 m-screen) and the 70 mesh screen $(70$ m-screen), adapted from [32] . . . . . . . . . . 17

2.5 A schematic of the problem simulated by reference [41], from [41] . . 18

2.6 The grid termination of the ejector used by reference [44] as seen from the inside of the ejector, from [44] . . . . . . . . . . . . . . . . . . . . 21 
$3.1 \quad$ An estimate of the anticipated sound pressure spectrum caused by the scale model ejector jet . . . . . . . . . . . . . . . . . 25

3.2 Jet impingement feedback phenomenon, adapted from [52] . . . . . . 26

$3.3 \quad$ First (a) and second (b) modes of edgetone oscillation, from [62] . . . 29

3.4 An un-stalled diffuser (left) and a fully stalled diffuser (right), adapted from [70] . . . . . . . . . . . . . . . . . . . . . . . . . . . . . . . . . . 31

3.5 The wall length of a conical frustum for use in equation 3.8 . . . . . . 33

$3.6 \quad$ Example of a pressure oscillation between two walls . . . . . . . . . . 35

3.7 Longitudinal, azimuthal and transverse oscillation in a duct, adapted

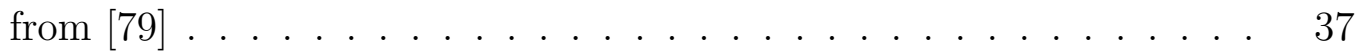

4.1 A schematic of the scale model (left) and the same schematic with components hidden (right) to allow the internal components to be visualized 46

4.2 Front (top left) rear (top right) and partial cutaway (bottom) view of the acoustic chamber as used in its nominal configuration, adapted from $\left[\begin{array}{l}82 \\ 6\end{array}\right]$

4.3 The predicted transmission loss for the anechoic chamber silencers, adapted from $[82] \ldots \ldots \ldots$

4.4 Labelled 3D CAD rendering of the collector assembly, from [82] . . . 51

4.5 The scale model mounted in the anechoic chamber, pictured without the flexible air hose attached . . . . . . . . . . . . . . . . . . . . . . . 52

$4.6 \quad$ The modified access panel for hose access with the external portion of the collector assembly visible . . . . . . . . . . . . . . . . . . 53

4.7 A schematic of the Pratt and Whitney High Speed Wind Tunnel, From [84] . . . . . . . . . . . . . . . . . . . . . . . . . . 54

4.8 A schematic of the Broom-Wade compressor, From [84] . . . . . . . . 55

4.9 Schematic of the HSWT layout, measurements in $\mathrm{mm}$. . . . . . . . . 58 
4.10 Schematic of the control system, adapted from [85] . . . . . . . . . . 59

4.11 A cross sectional schematic showing the location of total and static pressure readings used for velocity control . . . . . . . . . . . . . . . 60

4.12 Schematic showing the potential core of a free jet . . . . . . . . . 61

4.13 Schematic of the instrumentation setup . . . . . . . . . . . 65

4.14 Typical 4944-A microphone frequency response, from [88] . . . . . . . 69

4.15 The measurement uncertainty estimate for a range of atmospheric pressures . . . . . . . . . . . . . . . . . . . . . 73

4.16 Block diagram illustrating the steps required for Mach number calculation. . . . . . . . . . . . . . . . . . . 74

4.17 The measurement uncertainty estimate for a range of nozzle Mach numbers (with the nozzle measurements used for control) . . . . . . . 74

5.1 A schematic of the primary nozzle showing possible flow conditioners, spacers and contraction . . . . . . . . . . . . . . . . 80

5.2 Schematic of the scale model throat with flanged ends . . . . . . . . . 81

5.3 Schematic cross section of the two scale model diffuser components . 81

$5.4 \quad$ A schematic of the perforated cylinder, the left hand side interfaces with the diffuser and the right hand side accepts the cone . . . . . . . 82

$5.5 \quad$ An isometric schematic showing the front (left) and rear (right) of the

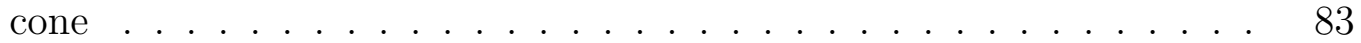

$5.6 \quad$ An isometric schematic of the base assembly . . . . . . . . . . . . . 83

$5.7 \quad$ A schematic of the primary nozzle weldment (left) and the fully assembled primary nozzle (right) . . . . . . . . . . . . . . . . . . . . . . . 85

$5.8 \quad$ A front (left) and side (right) view schematic showing the microphone enumeration ...................... . . . 88 
5.9 Schematic showing the velocity profile downstream of a pipe bend (left) and the secondary flows established at the outlet plane of the bend (right), adapted from [97] . . . . . . . . . . . . . . . . . . . . . . . . 92

5.10 Schematic showing the impact of probe tip shape and yaw angle, from [107] . . . . . . . . . . . . . . . . . . . . . . . . . . . . . . . . 98

6.1 Baseline Mach performance of the primary nozzle with the parameters suggested by Hall . . . . . . . . . . . . . . . . . . . . . . . . . . . . . 104

6.2 Steady state Mach performance of the primary nozzle with baseline parameters and the deadband set to zero . . . . . . . . . . . . . . 105

6.3 Mach performance for a variety of deadband compensator values . . . 107

6.4 Comparison of the Mach performance and valve supply voltage for a long duration run . . . . . . . . . . . . . . . . . . . . . . . . . 109

6.5 A portion of the comparison of the Mach performance and valve supply voltage . . . . . . . . . . . . . . . . . . . . . . . 110

6.6 A portion of the comparison of the Mach performance and derivative of the valve supply voltage . . . . . . . . . . . . . . . . . . . . . . . . 111

6.7 Impact of varying the supply pressure to the electro-pneumatic control valve on the Mach number unsteadiness . . . . . . . . . . . . . . . . . 112

6.8 Impact of varying PID control gains for "large" $K_{P}, K_{I}$ and $K_{D}$ values 113

6.9 Impact of varying PID control gains for "small" $K_{P}, K_{I}$ and $K_{D}$ values 115

6.10 Impact of a non-linear $K_{p}$ on Mach performance for a range of PID gains 117

6.11 Steady state analysis of the Mach performance for a non-linear $K_{P}$ of $15 / 0.5, K_{I}$ of 0.5 and $K_{D}$ of 0.5 . . . . . . . . . . . . . . . . . . . . 119

6.12 Steady state full-range Mach performance for a non-linear $K_{p}$ of 15/0.5, $K_{i}$ of 0.5 and $K_{d}$ of 0.5 . . . . . . . . . . . . . . . . . . . . . . . 120 
6.13 Values of Mach undershoot with the best fit polynomial used to predict and correct for the undershoot . . . . . . . . . . . . . . . . . . . . . . 122

6.14 Steady state Mach performance with non-linear PID gains and polynomial Mach undershoot correction . . . . . . . . . . . . . . . . 122

6.15 Relationship between the primary nozzle total pressure and the Pitotstatic total pressure . . . . . . . . . . . . . . . . . . 125

6.16 Static pressure ratio between the primary nozzle and Pitot-static probe for a range of Mach numbers . . . . . . . . . . . . . . . . . . . . . . . 126

6.17 A comparison of the primary nozzle Mach number measurement and Pitot-static Mach number measurement, showing a high correlation . 127

7.1 The calibration result obtained for microphone $1 . \ldots . . . . .131$

7.2 The noise floor obtained for microphone 1 . . . . . . . . . . . . . 131

7.3 Acoustic signature of the HSWT heater . . . . . . . . . . . . . . . . . 133

7.4 Acoustic signature of the HSWT dryer at 20, 30 and 35 psi tank pressure 134

7.5 Acoustic signature downstream of the silencer inside the chamber with the dryer emitting a tone . . . . . . . . . . . . . . . . . . . . . . . . . 135

7.6 Acoustic signature of the "valve noise" at Mach 0.9 . . . . . . . . . . 136

7.7 Acoustic resonance of the flexible hose $\ldots \ldots$. . . . . . . . . . . . . 137

\begin{tabular}{|ll|l|l|}
\hline 7.8 & Repetition of figure & 5.8 & A front (left) and side (right) view schematic \\
\hline
\end{tabular} showing the microphone enumeration . . . . . . . . . . . . . . 139

7.9 Acoustics in the secondary flow chamber \& anechoic chamber, baseline configuration, Mach 0.9 . . . . . . . . . . . . . . . . . . . . . . . . . 139

7.10 Acoustics in the lower outlet chamber, baseline configuration, Mach 0.9 141

7.11 Acoustics in the upper outlet chamber, baseline configuration, Mach 0.9 142

7.12 Acoustics behind the cone, baseline configuration, Mach 0.9 . . . . . 143 
7.13 The acoustic signature at location $\mathrm{C}$ at Mach 0.8 showing a clear tone and its octave . . . . . . . . . . . . . . . . . . . . . . . . . 144

7.14 Modal decomposition of the SPL plot for location F at Mach 0.9 . . . 145

7.15 The locations and directions of the traverses in the scale model with the coordinate system defined . . . . . . . . . . . . . . . . . . . . . . 147

7.16 Traverse results downstream of the primary nozzle . . . . . . . . . . . 148

7.17 Traverse results at the scale model inlet and outlet . . . . . . . . . . 148

7.18 The measured temperature of the primary nozzle wall and ambient for a long duration run at the design Mach number . . . . . . . . . . . . 150

\begin{tabular}{|l|l|l}
\hline 8.1 Repetition of figure & 5.8 & A front (left) and side (right) view schematic
\end{tabular} showing the microphone enumeration . . . . . . . . . . . . . . . . . . 152

8.2 The cones used to test the acoustic impact of surface roughness . . . 154

8.3 The SPL spectrum as measured at location $\mathrm{F}$ for all surface roughness cones . . . . . . . . . . . . . . . . . . . . . 155

8.4 The SPL spectrum as measured at location I for the cone with a closed

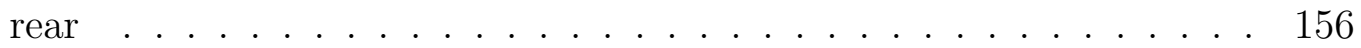

8.5 The estimation used for $S, V$ and $\alpha \ldots \ldots \ldots$. . . . . . . . . 157

8.6 The 3D printed cone with fins . . . . . . . . . . . . . . . . 158

8.7 The SPL spectrum as measured at location $\mathrm{F}$ for all fin cone configurations . . . . . . . . . . . . . . . . . . . . . . 159

8.8 The closed bottom cone . . . . . . . . . . . . . . . . . . . . . . . . 161

8.9 The SPL spectrum as measured at location F for the closed bottom cone 161

8.10 The elliptical cone . . . . . . . . . . . . . . . . . . . . . 162

8.11 The SPL spectrum as measured at location F for the elliptical cone . 163

8.12 The perforated cones, used for multiple tests . . . . . . . . . . . . . 164

8.13 The SPL spectrum as measured at location $\mathrm{F}$ for the perforated cones 165 
8.14 The $58 \%$ OAR perforated cone placed at the perforated cylinder midlength with the solid cone visible behind it . . . . . . . . . . . . . . 167

8.15 The SPL spectrum as measured at location D for the perforated cones placed at the midlength of the perforated cylinder . . . . . . . . . . . 167

8.16 The 33\% perforated cone placed "in the diffuser" (left) and a cross sectional schematic of this configuration (right) . . . . . . . . . . . . 169

8.17 The SPL spectrum as measured at location $\mathrm{F}$ for the perforated cones placed in the diffuser . . . . . . . . . . . . . . . . . . . . . . . . . . . 170

8.18 The reverse perforated cone within the perforated cylinder . . . . . . 171

8.19 The SPL spectrum as measured at location $\mathrm{F}$ for the reverse perforated cone within the perforated cylinder . . . . . . . . . . . . . . . . . . . 172

8.20 Schematic of the reverse perforated cone tested without the perforated cylinder . . . . . . . . . . . . . . . . . . . 173

8.21 The SPL spectrum as measured at location $\mathrm{F}$ for the reverse perforated cone tested without the perforated cylinder . . . . . . . . . . . . . . . 174

8.22 The SPL spectrum as measured at location $\mathrm{F}$ for perforated disks and perforated cones placed "in the diffuser" . . . . . . . . . . . . . . 176

8.23 The SPL spectrum as measured at location $\mathrm{F}$ for 3 perforated disks replacing the baseline cone . . . . . . . . . . . . . . . . . 178

8.24 The screen displaced from the diffuser inlet . . . . . . . . . . . . . . . 179

8.25 The SPL spectrum as measured at location $\mathrm{F}$ for the 3 types of screens placed at the diffuser inlet, all of which were damaged . . . . . . . . . 180

8.26 The SPL spectrum as measured at location $\mathrm{F}$ for the ejector without a diffuser sleeve . . . . . . . . . . . . . . . . . . . . . . . . . . . . . . 182

8.27 The rounded corners employed in the outlet flow chamber. . . . . . . 183 
8.28 The SPL spectrum as measured at location $\mathrm{F}$ for the outlet flow chamber with rounded corners . . . . . . . . . . . . . . . . . . . . . . . . . 184

8.29 The outlet flow chamber lined with acoustic foam and a close up view of the microphone protruding beyond the foam . . . . . . . . . . . . . 185

8.30 The SPL spectrum as measured at location $\mathrm{F}$ for the ejector walls lined with acoustic foam . . . . . . . . . . . . . . . . . . 186

8.31 The SPL spectrum as measured at location B for the ejector walls lined with acoustic foam . . . . . . . . . . . . . . . . . . . . 187

A.1 The microphone calibration results . . . . . . . . . . . . . . 207

A.2 The differential pressure transducer calibration results . . . . . . . . . 208

A.3 The atmospheric pressure transducer calibration results, data from Gi-

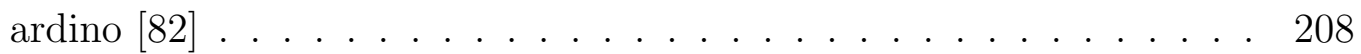

C.1 Acoustic signature of the "valve noise" for all Mach numbers . . . . . 211 


\section{List of Tables}

$3.1 \quad$ Fundamental Modes of the Secondary Flow Chamber . . . . . . . . . 38

3.2 Octaves of the Fundamental Modes of the Secondary Flow Chamber . 39

3.3 Fundamental Modes of the Outlet Flow Chamber . . . . . . . . . . . 39

3.4 Octaves of the Fundamental Modes of the Outlet Flow Chamber . . . 40

3.5 Fundamental Modes of the Throat . . . . . . . . . . . . . . . . 40

3.6 Longitudinal Modes of the Throat . . . . . . . . . . . . . . . . . . 41

3.7 Transverse Modes of the Throat . . . . . . . . . . . . . . . . . . . . . 41

3.8 Azimuthal Modes of the Throat . . . . . . . . . . . . . . . . . . . . . 41

$3.9 \quad$ Fundamental Modes of the Entire Ejector. . . . . . . . . . . . . . . . 43

4.1 Differential Pressure Transducers' Information . . . . . . . . . . . . . 66

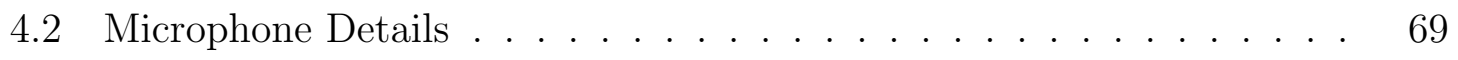

$4.3 \quad$ Values Required for the Uncertainty Analysis for the Atmospheric Pressure Transducer . . . . . . . . . . . . . . . . . . . . . . . . . . 72

5.1 Random-Incidence Absorption Coefficients of Materials Used in Full Scale and Scale Model Facilities, from [96] . . . . . . . . . . . . . . . 89

6.1 Average Measured Mach Number, Standard Deviation and Uncertainty Range for a Variety of Target Mach Numbers . . . . . . . . . . . . . 123

7.1 Microphones Locations in the Scale Model . . . . . . . . . . . . . . . 138

$8.1 \quad$ The Cone Roughness's Tested and Their Average Particle Size . . . . 154 
B.1 Roots of the Bessel Function Derivative . . . . . . . . . . . . . . . . . 209 


\section{Nomenclature}

\section{Abbreviations}

$\begin{array}{ll}\text { avg } & \text { Average (used only in plots) } \\ \text { B\&K } & \text { Brëul and Kjær } \\ \text { CAD } & \text { Computer Aided Design } \\ \text { CFD } & \text { Computational Fluid Dynamics } \\ \text { CNC } & \text { Computer Numerical Control } \\ \text { dB } & \text { Decibel } \\ \text { dBA } & \text { A-Weighted Decibel } \\ \text { G1S } & \text { Good One Side } \\ \text { HSWT } & \text { High Speed Wind Tunnel } \\ \text { Hz } & \text { Hertz } \\ \text { ID } & \text { Inner Diameter } \\ \text { OAR } & \text { Mach Number (used only in plots) } \\ & \\ & \\ \text { Horea Ratio }\end{array}$


OASPL Overall Sound Pressure Level

OD Outer Diameter

PID Proportional Integral Derivative

psi Pounds per Square Inch

psi(a) Pounds per Square Inch (Absolute Pressure)

psig Pounds per Square Inch (Gage Pressure)

SPL Sound Pressure Level

\section{Symbols}

a Calibration coefficient of slope, equivalent to microphone sensitivity

A Cross sectional area of a honeycomb cell

b Calibration coefficient of offset

c $\quad$ Speed of sound

$C_{A} \quad$ Phase velocity of the feedback acoustic wave

$C_{e} \quad$ A constant used in the edgetone calculation

$C_{I} \quad$ Phase velocity of the downstream propagating instability wave

d diameter

DI Directivity index of jet noise 
e Product of the surface area of an enclosure and the absorption coefficient of the surface

ER Entrainment ratio

f Frequency

F A calculated value

G A proportionality constant

$\mathrm{h} \quad$ The distance between a jet and an edge

i Longitudinal mode of oscillation

I Acoustical power coefficient

j Transverse mode of oscillation

$\mathrm{J} \quad$ The distance between a nozzle and a wall

$\mathrm{k} \quad$ Azimuthal mode of oscillation

$K_{D} \quad$ Derivative gain

$K_{I} \quad$ Integral gain

$K_{P} \quad$ Proportional gain

1 Length of the ejector duct

L Distance between two walls

M Jet Mach number 
$\dot{m} \quad$ Mass flow rate

n Mode of oscillation

O Overall sound power level

p Perimeter of a honeycomb cell

P Pressure

q A constant used in the edgetone calculation

$r \quad$ Distance from the jet orifice to the point of observation

$\mathrm{R}$ Radius of the base of the cone

$R_{e} \quad$ Reynolds Number

S Cross sectional area of a Helmholtz resonator neck

S.F. Scale Factor

St Strouhal Number

t $\quad 60 \mathrm{~dB}$ reverberation time

T Absolute temperature

u Velocity

v Volume contained within a Helmholtz resonator

V Voltage

W Power 
$\mathrm{x}$ Distance along the centerline of the cone

y Distance from the axis of rotation of the ellipse to the surface of the ellipse

$\alpha \quad$ The length of the neck of a Helmholtz Resonator

$\beta \quad$ Length of the diffuser wall

$\gamma \quad$ Ratio of specific heats

$\delta \quad$ Uncertainty in a measured value

$\eta \quad$ Acoustical efficiency

$\rho \quad$ Density

$\sigma_{j k} \quad$ The $\mathrm{j}^{t h}, \mathrm{k}^{t h}$ root of the Bessel function derivative

\section{Subscripts}

0

$\infty$

a

anm

$\operatorname{avg}$

baseline

cell
Total condition (as in total pressure)

Free stream

Acoustical

Acoustic natural modes

Average value

Of the baseline geometry

Of a honeycomb cell 


\begin{tabular}{|c|c|}
\hline cone & Of the cone \\
\hline diff & Diffuser \\
\hline dist & Distortion value \\
\hline duct & Of the ejector duct \\
\hline edge & Of the edgetone \\
\hline eff & Effective duct length \\
\hline encl & Of an enclosure \\
\hline full scale & Of the full scale conditions \\
\hline Helmoltz & Of the Helmholtz resonator \\
\hline imp & Impingement \\
\hline $\mathrm{i}$ & Longitudinal \\
\hline j & Transverse \\
\hline $\mathrm{J}$ & Of the jet \\
\hline $\mathrm{k}$ & Azimuthal \\
\hline $\mathrm{m}$ & Mechanical \\
\hline $\max$ & Maximum value \\
\hline $\min$ & Minimum value \\
\hline
\end{tabular}




$\begin{array}{ll}\text { new } & \text { Of the new geometry being tested } \\ \text { primary } & \text { In the primary stream } \\ \text { ref } & \text { Reference } \\ \text { RMS } & \text { Root mean square } \\ \text { S } & \text { Sampling } \\ \text { S } & \text { Schroeder } \\ \text { secondary } & \text { In the secondary stream } \\ \mathrm{x} & \text { In the } \mathrm{x} \text { direction } \\ \mathrm{y} & \text { In the y direction } \\ \mathrm{z} & \text { In the } \mathrm{z} \text { direction }\end{array}$




\section{Chapter 1}

\section{Introduction}

Ejectors, also commonly referred to as eductors ${ }^{1}$ or jet pumps, are used to transfer energy from a primary stream of fluid to a secondary stream which can be gaseous, liquid, powder, slurry, or any mixture of the above. In a general sense, they are used for pumping, mixing and transferring heat. Their versatility, simplicity, reliability (as there are no moving parts), and low cost make them appealing, however, this is offset by low efficiency stemming from losses due to mixing and friction [1]. Ejectors can be found in such diverse applications as wind tunnels [2, 3], propulsive devices [4, 5], jet engine silencers and thrust augmenters [6, 7], gas-dynamic lasers [8], engine and rocket tests [9, 10], aerators in wastewater treatment systems [11], fuel cells [12, 13], and - perhaps most commonly found in academic literature - in refrigeration (see the good review by Elbel and Lawrence [14]), to name a few. As a consequence of their wide range of applications, ejectors come in a wealth of shapes and sizes. Figure 1.1 shows both a large and small ejector demonstrating this variability.

\footnotetext{
${ }^{1}$ The term eductors usually applies to low pressure ejectors
} 


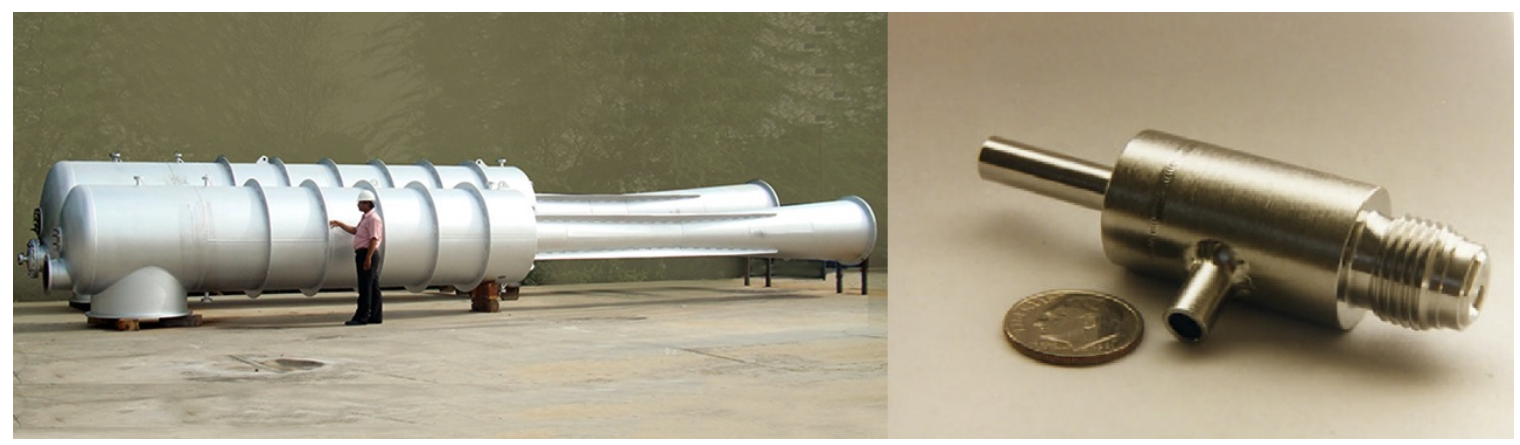

Figure 1.1: A large steam ejector (left, from [15]) and a mini eductor (right, from [16])

\subsection{Ejector Description and Basic Principle of Op-}

\section{eration}

The wide range of applications for ejectors leads to a wide range of terminology, with each term specific to its particular application. In its simplest form an ejector is nothing more than a fluid jet exhausting into a duct. Certain geometrical modifications to this have led to what is now commonly referred to as an ejector. Figure 1.2 shows a schematic cross section of an ejector with the nomenclature used throughout this work, mostly similar to that of reference [17].
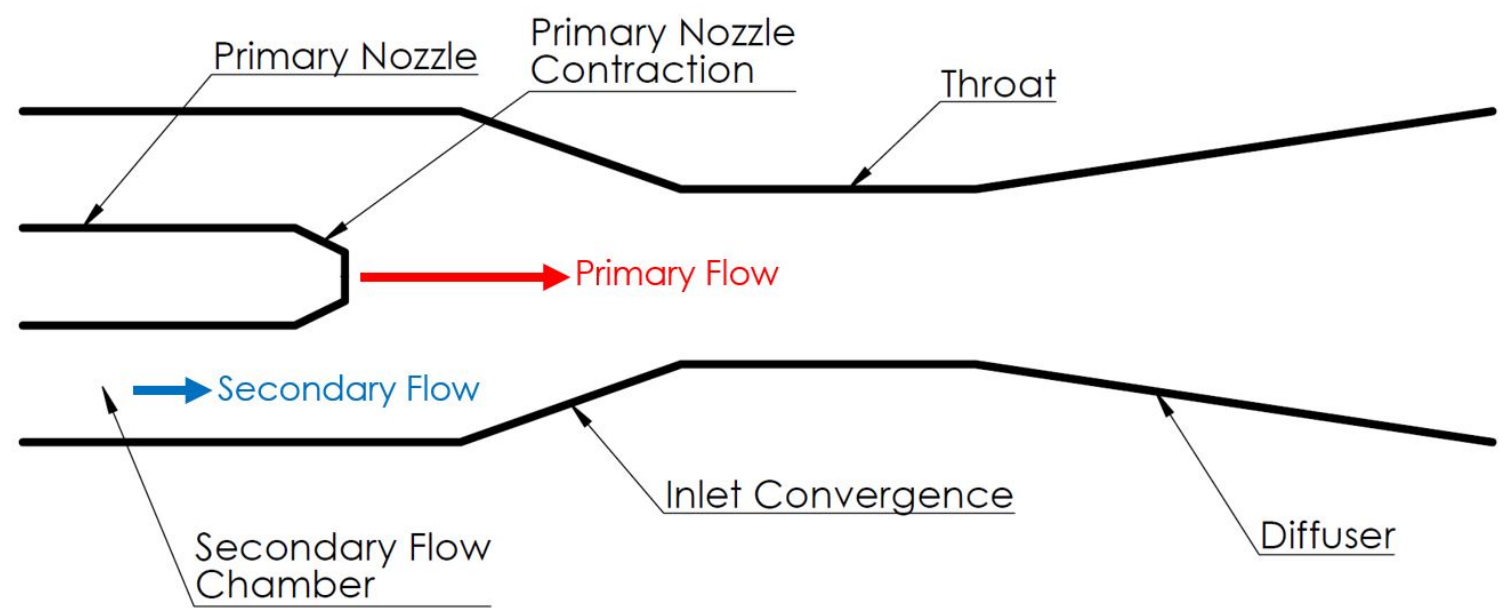

Figure 1.2: An ejector schematic outlining the major components 
No matter which purpose an ejector may be used for, all ejectors follow the same basic principle of operation. The operation relies on the Venturi effect, namely that a drop in fluid pressure is caused by an increase in velocity. The primary nozzle (sometimes called the motive nozzle) contains a high pressure fluid which accelerates in the primary nozzle contraction - referred to simply as the contraction from here on - and flows into the ejector body. The resulting low pressure stream entrains the surrounding fluid thus causing a downstream flow in the secondary flow chamber (sometimes called the suction chamber). Both streams flow through the inlet convergence and into the throat (often called the mixing section, mixing chamber or duct). In the throat, mixing occurs through exchange of mass momentum and energy [18]. The resulting mixed flow then undergoes a pressure recovery in the diffuser before being exhausted. This exhaust is often to a pipe, a reservoir or simply to atmosphere. In this work, the diffuser and everything downstream of it is considered the aft portion of the ejector.

Any of the components mentioned above can be circular or rectangular and there are ejectors which make use of a combination of both. It is important to note that many ejectors employ a supersonic primary stream due to the very low pressure resulting from a supersonic stream. In these cases, the primary nozzle does not employ a convergence but rather a converging-diverging nozzle section. The flow physics of ejectors is not the topic of this research thus a detailed explanation of the flow physics (shear layers, mixing mechanisms and etc.) is not presented, however, there is significant literature on the topic (see reference [18] for example).

An important performance parameter for ejectors is the entrainment ratio (ER). The entrainment ratio is the ratio of mass flow, $\dot{m}$, of the primary stream to the mass flow of the secondary stream. It can be defined as 


$$
E R=\frac{\dot{m}_{\text {primary }}}{\dot{m}_{\text {secondary }}}
$$

Changes in ejector geometry can have an impact on the entrainment ratio (net mass flow rate), however, these changes stem from changes to the secondary or entrained mass flow. The primary stream generally has a fixed mass flow rate, thus a drop in entrainement ratio is the result of a net mass flow rate decrease in the secondary stream.

\subsection{Motivation and Problem Definition}

Although ejectors can be of great use, there are issues associated with the noise they can generate. In some cases, ejectors are used to help silence otherwise loud noise sources (see reference [19] for example), however, there are other cases where ejectors themselves are important sources of noise (see reference [20] for example). This noise is of course dependent on many factors, potentially including the primary jet velocity, the jet to the inlet convergence distance, the throat length and diameter,

and the noise measurement location. Reference [21] performs tests where the primary nozzle position relative to the inlet convergence leads to a 5 decibels $(\mathrm{dB})$ difference in the measured far field noise. In addition, for certain primary nozzle positions discrete tones are measured within the throat. This is true even for nozzle Mach numbers well below unity (Mach 0.6) where the tones cannot be attributed to sonic phenomena such as screech tones.

The frequency of the noise generated by ejectors appears to correlate with their size, thus large ejectors tend to produce low frequency noise. For some facilities, a large amount of undesirable - and often unacceptable - infrasound is generated. Infrasound is generally accepted to be noise at frequencies below the threshold of 
human hearing of $20 \mathrm{Hertz}(\mathrm{Hz})$. In this work, low frequency noise is considered to be any noise below $100 \mathrm{~Hz}$. The noise generated by ejectors is believed to be due to some form of aeroacoustic pressure pulsations but the exact noise generation mechanism(s) is not known.

Not only can low frequency noise cause damage to buildings and infrastructure due to the air pressure pulsations, but it can also have a significant impact on human health and well-being. Although the effects of infrasound on human health is a controversial subject, reference [22] presents a summary of literature of the potentially adverse effects of infrasound exposure in humans which include 2 vertigo, imbalance, intolerable sensations, incapacitation, disorientation, nausea, vomiting, bowel spasms, resonance in inner organs (such as the abdomen and heart), depression, drowsiness, central nervous system effects, cardiovascular effects and respiratory effects to name a few.

\subsection{Research Objectives and Methodology}

The objective of this research is to further the level of understanding of low frequency noise being generated in ejectors and to find methods to reduce it. This is done through theoretical predictions as well as by experimental tests conducted using a scale model ejector. Experiments are conducted with two primary objectives. The first is to attempt to further the understanding of noise generation in ejectors. The second is to find practical solutions to reduce this noise.

An emphasis is placed on investigating the aft portion of the ejector as this is believed to be the source of unwanted noise. A common air handling silencer or

\footnotetext{
${ }^{2}$ While these symptoms are present in some people, others may show no symptoms under identical circumstances. Different studies have concluded opposing findings and the subject of infrasound exposure in humans is ongoing.
} 
"duct cap", shown in figure 1.3, is added downstream of the diffuser in an attempt to silence the ejector. This duct cap, refered to as the silencer in the remainder of this text, comprises of an upstream facing cone placed at the rear of a perforated cylinder ${ }^{3}$ This configuration of combined ejector and silencer is considered the baseline to which modifications are made.

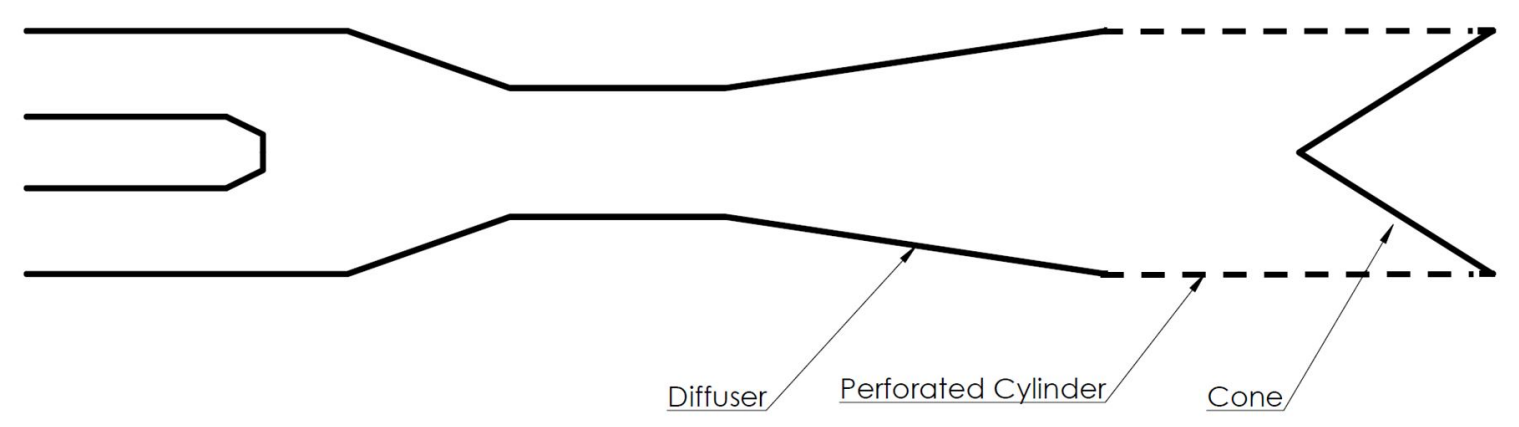

Figure 1.3: An ejector schematic showing the baseline silencer added aft of the diffuser

The use of a scale model ejector allows for tests to be done rapidly and inexpensively. The methodology employed for testing new silencer configurations is varied. Some configurations mimic solutions presented in the literature, some are chosen based on personal judgement and others are opted for as the result of previous tests. Many configurations are tested simply to observe the outcome, in an attempt to discover which ejector parameters may affect acoustics. The configurations are tested in order of estimated efficacity, simplicity, and ease of implementation. Many modifications are made in an attempt to reduce the noise levels of the ejector. The configurations tested fall into one of two general categories. They are either configuration changes which are practical to implement on the full scale or as configuration changes which are not. The former serves the purpose of finding a configuration which can be put into place to reduce noise whereas the latter configurations are used in an attempt

\footnotetext{
${ }^{3}$ This type of silencer can be found in reference [23], section 6 , diagram 11-17
} 
to understand the noise generation mechanisms without practical importance.

\subsection{Scope and Limitations of Work}

It is worth pointing out that nearly everything presented in this thesis could be the subject of its own in-depth analysis. It is also important to discuss the limitations of the work as the results may not be directly applicable to a full scale facility. Some of the experiments performed cannot easily be scaled given the range of sizes found in ejectors. For example, changing the diffuser to a profiled shape with fins and perforations is easy on a small scale model, however, when scaled up to full scale the logistics and costs of manufacturing and maintenance become important. The same is true of a scaled down version. Small scale manufacturing can be quite difficult and costly to implement.

This work also limits itself to the subsonic air-air ejector, principally for two reasons. The first reason is that the ejector scale model is representative of a facility which itself does not employ supersonic flow. Secondly, the flow physics of a supersonic ejector become significantly more complicated due to the expansion of the jet, potential for choked flow and creation of shock waves. A subsonic analysis is thus undertaken before attempting to understand the more complex supersonic case.

Computational AeroAcoustics (CAA) presents certain advantages to experimental tests and could be a good alternative in investigating ejector noise, however, CAA is incredibly computationally expensive and not currently used at Carleton University. Given Carleton's extensive aerodynamic facilities, experimental investigations are viewed as the best technique at this time.

Much of the work undertaken in this thesis deals with intellectual property and for this reason many plots and figures must be presented in a less than ideal manner. 
Non-dimensionalization, omission of exact values and omission of certain details are some of the techniques used to protect this intellectual property. Despite this, it is believed that the overarching results are sufficiently clear and presented in such a way to be well understood.

\subsection{A Note on Units}

Both the metric and imperial systems of units are used in this text. Canada is in a special situation and still uses both systems of units after the change from the imperial system to the metric system in 1970. Certain applications have retained the old system whereas other uses have switched over to SI units. For example, human height is typically measured in feet but distance travelled in kilometers. Most engineering calculations for this thesis are performed in the SI system of units, however, commercial equipment is often sold with imperial specifications, partially due to imports from the United States. The building supplies and raw materials used for portions of this project are sold in imperial dimensions thus the design is done in this system of units. Throughout the text dual units are used to avoid potential confusion. 


\section{Chapter 2}

\section{Literature Review}

\subsection{General Reports}

Given the use of ejectors in a wide range of applications, ejector noise can be found across a wealth of industries, however, reports of ejector noise are somewhat rare. This section presents the findings from non-aerospace literature pertaining to ejector noise. Croll Reynolds! discusses the noise generated from the discharge of high velocity gasses from an ejector. The use of a silencer which can be added to the aft end of an ejector which may achieve up to $\left.30 \mathrm{dBA}\right|^{2}$ reduction in noise level is mentioned as is the fact that this is standard procedure for hogging or priming ejectors. The company "dB Noise Reduction" also promotes the sale of their steam silencers to reduce unacceptably high noise levels which can be a result of steam ejector discharge and exhaust ${ }^{3}$. Two abstracts pertaining to ejector noise, references [24, 25], mention modifications made to ejectors in order to observe the impact on noise generation. These references find differences of $50 \mathrm{~dB}([24])$ and "a considerable reduction in noise level" ([25]), however, the full details are not available given that these are only

\footnotetext{
${ }^{1}$ available at http://croll.com/library/technical-articles/ noise-control-for-steam-ejectors/

${ }^{2} \mathrm{dBA}$ is a weighted form of $\mathrm{dB}$ meant to better represent the human sensitivity to noise

3 available at http://www.dbnoisereduction.com/vent_silencers/steam_silencers.php
} 
abstracts. There is also the work found in reference [26] which outlines the testing on a few different types of steam ejectors used to heat water. It is found that the ejector used has an impact on the generated noise, however, this report is of a practical nature and does not examine the reasoning of why certain ejectors are quieter.

\section{$2.2 \quad$ Ejector Noise In the Aerospace Industry}

The one industry in which the noise of ejectors has been given more attention is the aerospace industry. The use of ejectors to change the noise properties of fluid jets is the most reported in literature. The author believes there are a few reasons why the aerospace industry has been the main investigator of ejector noise and why other industries have not:

1. The noise generated in industrial settings would most likely be addressed without the creation of scientific literature (journal publications and etc.)

2. In-depth studies of the noise generation of ejectors requires a knowledge in aeroacoustics and specialized instrumentation, both of which already exist within the aerospace community

3. The mass flow and velocity from jet engines is quite large causing large amounts of noise (acoustic power scales to the $8^{\text {th }}$ power of velocity and the $2^{\text {nd }}$ power of jet diameter, see chapter 5 , equation 5.2) as opposed to industrial applications which may encounter lower mass flows and velocities

4. The aerospace industry is a highly engineered industry and there is a need for reduced noise emissions

5. The study of noise generated by ejectors is often a secondary finding from other (aerodynamic) tests, such as in reference [27] where the acoustic interactions 
were "observed by chance".

For these reasons, nearly all work reported in the literature relates to ejectors and jet noise. This section presents relevant literature from the aerospace industry pertaining to noise generated by ejectors. Experimental work is presented first, followed by computational work and two special cases pertaining to the end configuration (silencer).

\subsubsection{Experimental Work}

The work of Middleton [19] from 1963 is one of the first focusing on ejector noise. Middleton cites only 5 ejector noise references all of which are from the late 1950's and early 60's, which coincides well with the advent of commercial aviation. It is outlined that these references mostly test a combination of ejector with a special noise reducing nozzle, and thus do not focus purely on the noise generated by the ejectors themselves. Middleton uses an axisymmetric 2 inch $(50.8 \mathrm{~mm})$ diameter primary nozzle to evaluate the noise generated by ejectors of 3 different diameters $(1$, 2 and 3 primary nozzle diameters, or simply nozzle diameters), with each diameter having 5 length possibilities (3, 6, 12, 20 and 32 nozzle diameters). Each ejector has a 1 inch $(25.4 \mathrm{~mm})$ radius bellmouth inlet. A schematic of the test setup is shown in figure 2.1. It is found that no excessive noise is recorded perpendicular to the ejector walls meaning that the noise generated does not emanate from the sides of the ejector (through mechanical vibrations for example). It is found that some noise is generated at the ejector inlet (convergence). For this reason, an "intake box" is used to isolate the intake noise from the efflux noise. It is found that at the efflux, discrete frequency noise is strongly generated for the subsonic case with shorter ejectors causing louder tones with up to a $20 \mathrm{~dB}$ sound pressure increase over 
the broad-band noise range. The broadband noise emitted from the ejectors is found to be independent of diameter but to decrease with increasing length, attributing an importance to mixing conditions and length of the ejector as opposed to the outlet velocity.
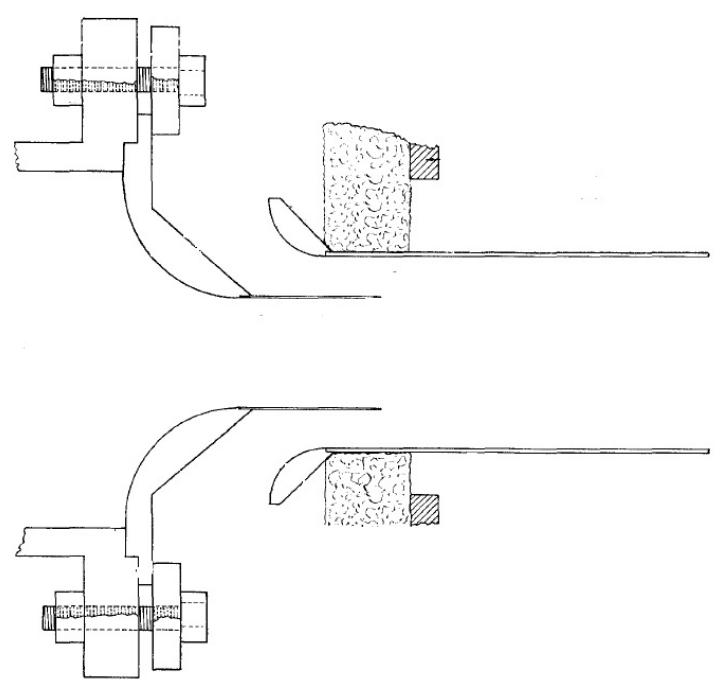

Figure 2.1: A schematic of the test setup used by Middleton, adapted from [19]

Reference [28] from 1968 observes the generation of tones in a variety of ejector shrouds and names these tones "howling". Interestingly, 3 geometrically similar cases are tested (including a full scale jet engine test) and only the smaller 2 of the 3 howled for the same flow conditions. This effect remains unexplained. The howling is attributed to natural acoustic modes of the shroud and the frequency of resonance is found to depend on the jet velocity. The generation of discrete tones non-linearly depends on jet velocity, which is indicative of a modification of the ejector flow caused by sound waves. Howling can unreliably be suppressed by modifications to the ejector outlet, such as the insertion of a lobed nozzle.

References [27, 29 31] from 1975, 1977, 1981 and 1988 respectively, study the impact of the generated noise on the mixing characteristics of ejectors without the 
noise being a primary focus. The research also focuses mainly on supersonic jets. Despite these deviations from the experimental work contained in this thesis, the conclusions drawn can be of interest. It is suggested that the duct resonant frequencies are the source of noise and that, in this case, the screech tones (small disturbances amplified by the jet velocity field causing the jet to oscillate on a large scale emitting noise [30], purely a supersonic phenomenon) of the jet couple with the duct modes in a discontinuous fashion. There is no reason to believe that in the subsonic case another acoustic source could not couple with the duct modes in a similar discontinuous fashion. Reference [31] finds that at lower frequencies, the tone emitted by ejectors coincides with the symmetric natural modes of the throat. Schlieren photography, a technique where local differences in fluid density caused by flow can be visualized and photographed, is employed on ejectors emitting discrete tones. Figure 2.2 shows the schlieren photograph of both the resonant and non-resonant case. Interestingly, the resonance occurs at a lower jet velocity than the non-resonance. As can be seen, the flow mixing in the duct is greatly altered by the acoustic resonance. The photographs help establish that the (supersonic) jet oscillates in a helical fashion under resonant conditions.
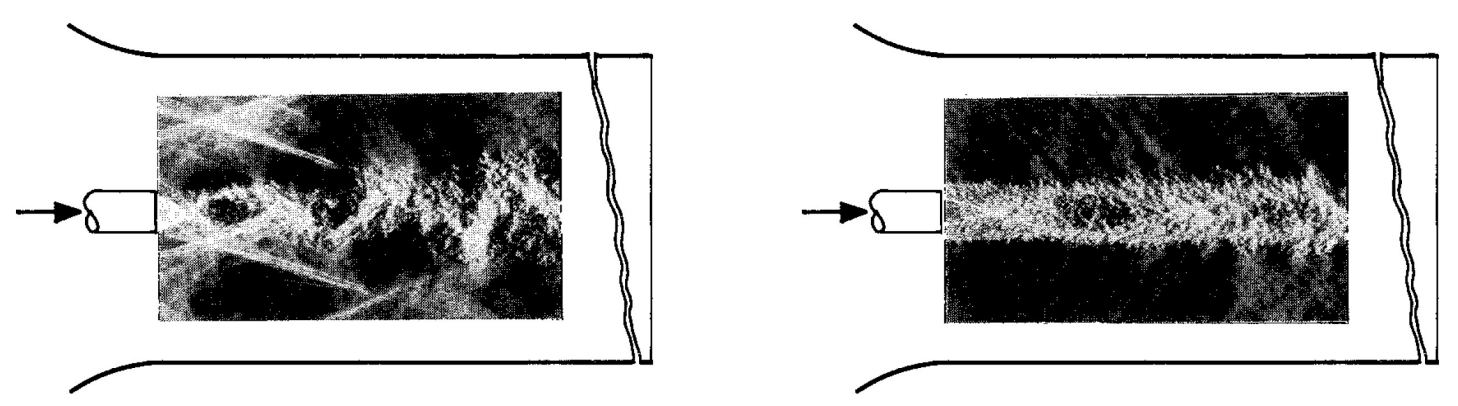

Figure 2.2: The Schlieren photos taken by reference [31] of a resonant (left) and stable (right) ejector flow, adapted from [31]

Reference [20] from 1995 investigates the generation of discrete tones in supersonic 
ejectors - in much the same way as [31] - but with a rectangular ejector and an emphasis placed on understanding the mechanism of tone generation. The tones are found to be as much as $20 \mathrm{~dB}$ greater than the broadband noise and as the area ratio between the ejector and primary nozzle is decreased (smaller ejector throat for fixed nozzle size) more tones appear, with the additional tones being related to the duct modes. A very clear schlieren photograph, shown in figure 2.3, is taken showing an ejector with both the transverse and organ pipe (longitudinal) resonant modes present. These modes, the two dominant modes, are linked to the acoustic spectrum and can explain most tonal peaks observed. The peaks are found to match well with either the longitudinal mode, the transverse mode or any linear integer combination of the two. The acoustic modes are believed to be established by the oscillation of the jet, which itself has specific modes. When the jet mode matches or is near to an ejector mode the jet mode "locks on" to the ejector mode and strong tones are heard. The symmetric and antisymmetric modes of the jet are found to coincide with the longitudinal and transverse modes of the ejector duct.

The conclusion that a propagating instability wave in the shear layer of the jet creates a feedback loop with upstream propagating acoustic waves is used to explain the staging of screech tones. In other words, at a given jet velocity an acoustic disturbance is created in the shear layer. This disturbance grows as it moves downstream. Eventually this disturbance causes an acoustic wave which propagates upstream. Upon reaching the jet, this wave creates another disturbance locking the jet into a resonant feedback loop. It is found that the ejector mixing is not affected by these tones except when the ejector dominant tone matches the most unstable Strouhal number a non-dimensionalized frequency - of the primary jet.

Reference [21] from 1996 performs experimental tests on an ejector to determine 


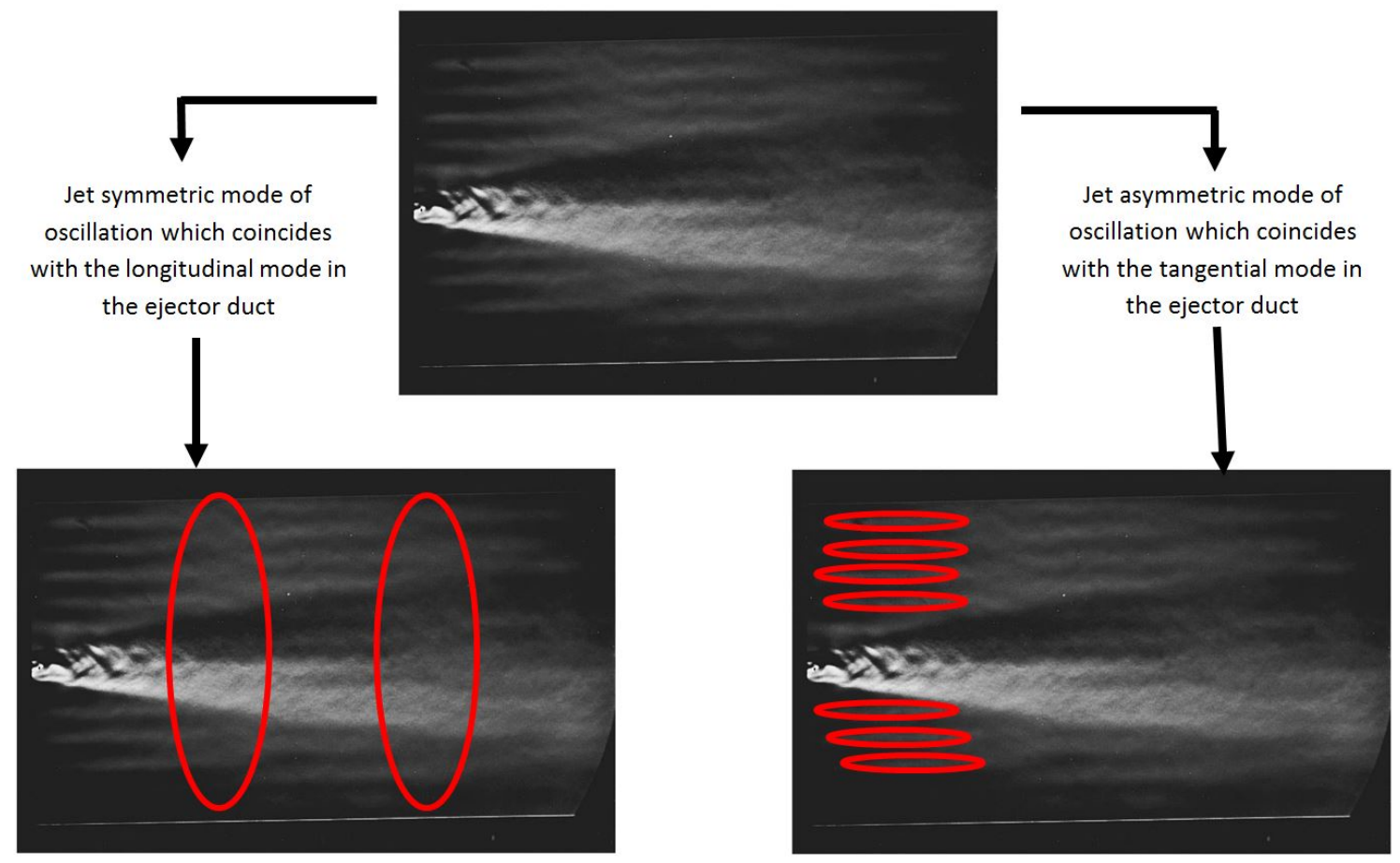

Figure 2.3: A Schlieren photograph taken by reference [20] of a resonant ejector flow highlighting the longitudinal and transverse oscillations present, adapted from [20]

the effect that mixing has on the broadband noise generation, as measured in the far field. Although the majority of tests are performed with a lobbed nozzle and some at supersonic speeds, it is found that placing the nozzle closer to or into the primary contraction can lead to an increase of $5 \mathrm{~dB}$ in the far field noise. Discrete tones equivalent to those of the transverse resonance of the duct are found for the majority of the test conditions. The use of a throat lined with attenuating foam (helps to prevent natural modes) leads to the conclusion that the mixing noise in the throat mostly causes high frequency noise (above $5 \mathrm{kHz}$ in this case) whereas the far field low frequency noise (below $5 \mathrm{kHz}$ ) is dominated by the low frequency noise associated with the larger diameter ejector outlet jet flow. It is found that the high frequency noise is seemingly generated at the primary nozzle exit whereas the low frequency noise is generated a certain distance downstream of the ejector outlet. 
Reference [32] from 2013 undertakes a series of tests which could be viewed as simplified ejector tests. In essence, a high speed air jet is blown into a section of pipe (a duct). The jet Mach number, the jet to duct distance, duct length and duct diameter are varied in a parametric manner to observe their impact on noise generation. In addition, certain techniques are used in an attempt to quiet the acoustics of the duct. This paper is densely packed with information and its reading is highly recommended for full comprehension but it can be summarized as follows: The source of unwanted noise is attributed to the duct resonant modes, with mode staging (non-linear jumps to different frequencies, often separated by "dead zones") occurring. The jet can couple to a duct resonant mode when the jet preferred mode instability matches the duct resonant mode, causing a high amplitude discrete frequency tone. This coupling occurs for ducts less than 3 primary nozzle diameters in diameter and within the 1-5 length-to-diameter ratid ${ }^{4}$. When this coupling is not present, large amounts of broadband noise may be generated due to the jet exciting a range of duct modes. Longitudinal fins, tabs and a rod placed perpendicular to the flow are found to be ineffective at suppressing noise, with the exception of the perpendicular rod being effective in the discrete tone jet coupling case. A screen placed at the aft of the duct is found to be quite effective at reducing both broadband and tonal noise and the proposed mechanism of this reduction is the damping of pressure nodes of longitudinal modes of the duct. In certain situations, the screen is found to reduce the noise by $25 \mathrm{~dB}$. Interestingly, the impact of the screens on the entrained airflow is not mentioned. There is however a mention that holes are made in the 70 mesh screen because the blockage would be too high without them. The decrease in entrainment, thus likely duct outlet velocity, is not discussed. Note that reference [33] discusses the relationship between flow rate and noise generation and finds that the noise reduction

\footnotetext{
${ }^{4}$ It is hard to compare this criteria with previous works such as in [20] due to the use of a rectangular ejector although the proposed mechanism is essentially the same
} 
caused by an ejector nozzl 55 compared to a free jet is directly linked to the velocity decrease caused by the flow blockage (recall that the noise generated is approximately a function of the $8^{\text {th }}$ power of jet velocity).
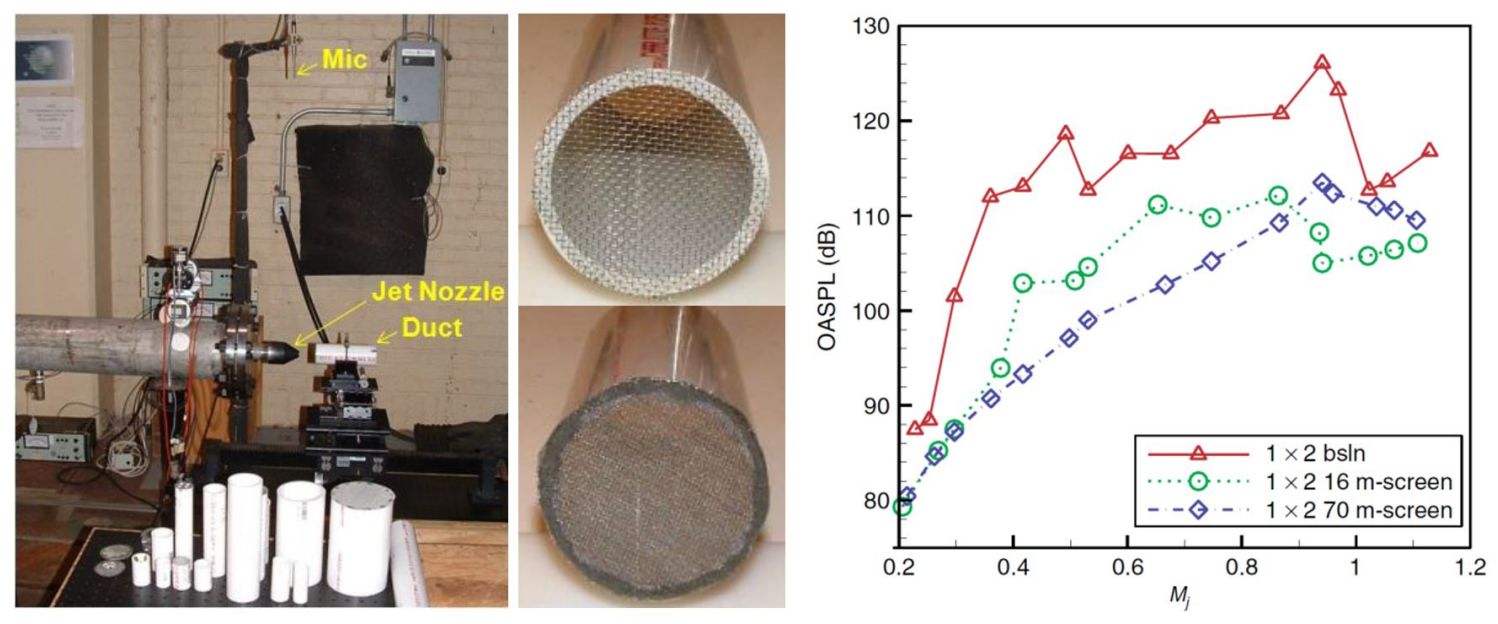

Figure 2.4: The test setup used by [32] (left), with the two screens tested providing good noise suppression (center), and the resulting drop in overall sound pressure level for the baseline (bsln), 16 mesh screen (16 m-screen) and the 70 mesh screen (70 m-screen), adapted from [32]

The work presented by reference [32] is seemingly what leads to US patent number 8,939,253 B1 [34], filed by the same authors. It outlines a method for reducing the noise from a jet discharging into a duct and makes use of "damper members" placed at the fore or aft end of said duct.

There exist other works relating to the noise generation in ejectors, however, these are mostly related to supersonic aircraft. For this reason, the flows tested are nearly all supersonic and make use of lobed type nozzles which greatly change the baseline acoustics and flow field of the jet. The interested reader is encouraged to consult references [35[38] for further information.

\footnotetext{
${ }^{5} \mathrm{~A}$ nozzle used to eject parts, not to be confused with ejector as used in this thesis
} 


\subsubsection{Computational Work}

As mentioned in the introduction, the use of computational techniques to study acoustic phenomena is difficult and highly computationally expensive. For this reason its use has been somewhat limited until recently. There does exist a limited number of reports of computer simulations used to investigate ejector noise. They are as follows.

Reference [39] from 1996 investigates ejector noise computationally. Although the papers' emphasis is on the details of the computational study, the Large Eddy Simulation results are presented but they are not discussed in depth. It is found that a Kelvin-Helmholtz instability is generated at the nozzle and that it grows as it moves downstream. Upstream propagating instability waves are also found. Interestingly, it is found that there are no noticeable reflections from the ejector outlet, contradicting the work of reference [40] discussed shortly.

A series of publications from the University of Illinois in 2007, 2008 and 2010, references [40 42] respectively, make use of computational tools to study the noise generated in ground based jet engine tests, essentially a large jet exhausting into a duct which can be viewed as a simplified ejector. Figure 2.5 shows a schematic of the problem being computationally investigated. An understanding of the resonance coupling and/or noise generation mechanism is of primary purpose of the work.

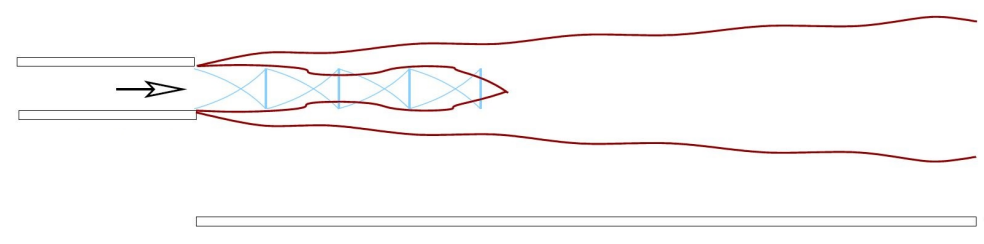

Figure 2.5: A schematic of the problem simulated by reference [41], from [41]

Reference [41] performs Direct Numerical Simulation on a 2 dimensional idealized 
geometry where the duct width and length are 5 times the primary flow width and with a flow velocity of Mach 1.5. It is found that "the hydrodynamic pressure associated with the vortical disturbances exiting the duct couples with pressure modes of the duct to close the feedback loop by exciting instabilities at the inflow (the nozzle)". This work also makes use of the Rocflu code for aeroacoustic simulations but it is found that it is too dissipative for reliable simulation of this case. Reference [40] investigates the sharp trailing edge of an ejector duct where some of the flow energy is converted into acoustic waves. It is proposed that these waves can travel upstream causing the coupling, and it is found to be possible given the correct conditions. It is also found that the shroud exit may not cause resonance at certain Mach numbers (a dead-zone phenomenon). This mechanism outlined may be valid, however, it does not address the case of ejectors without a sharp trailing edge where noise is still generated. Finally, reference [42] simulates a 2 dimensional Mach 1.2 under-expanded jet discharging into a duct, a Mach 1.2 jet with free discharge, and a Mach 1.5 jet discharging into a duct. When symmetry is imposed on the ducted Mach 1.2 jet, high amplitude pressure fluctuations (resonance with a tone) are observed, however, without symmetry imposed the tone goes away although large hydrodynamic fluctuations are present at the rear of the duct. In comparing the ducted and non-ducted Mach 1.2 jets, it is found that the jet frequency shifts in the ducted case and the noise amplitude changes from $138 \mathrm{~dB}$ to $159 \mathrm{~dB}$. This shift corresponds well with the work of reference [32] where the jet preferred frequency is found to shift in order to lock on to the ejector throat natural frequencies. Although these three computational studies propose advancements in the understanding of the discrete tone mechanism, the detailed mechanism - similar to that proposed previously - involves supersonic flow phenomena and provide no details on subsonic tones or broadband noise generation. 


\subsubsection{End Configuration}

Two final works worth mentioning are references [43, 44]. Reference [43] outlines scale model tests of a facility in which problematic noise is encountered. The ejector termination is similar to that used in this thesis - that is a perforated cylinder terminating with a front facing cone - and modifications are made to this setup to reduce the problematic noise. It is found that a $50^{\circ}$ forward facing cone leads to a $68 \%$ reduction in pressure fluctuations as compared to the baseline concave dish. It is also shown how the end termination is important to noise generation and any termination which is not concave provides an advantage to the concave setup.

Reference [44] uses an ejector to silence the noise from military engines. The ejector again makes use of an end configuration somewhat similar to that used in this work. Although a large portion of this work involves supersonic flow, it is found that the addition of the ejector can silence the noise levels of the engine by more than $20 \mathrm{dBA}$ in the $6-20,000 \mathrm{~Hz}$ range. Noise measurements made in the near as well as far field show interesting decreases in noise generation by using the ejector. An interesting finding is that the use of a cone as termination is more effective at suppressing noise compared to a pipe grid configuration (figure 2.6), however, this comes at the cost of increased flow restriction. The use of a semi-perforated cone also appears to have been used but details are not provided.

\subsection{Closing Remarks}

As can be seen, the work on ejector noise is somewhat random and sporadic. Ejector noise is not a "classic" field of study like jet noise which is well established with a solid understanding of noise generation mechanisms and abundant literature. Reports on 


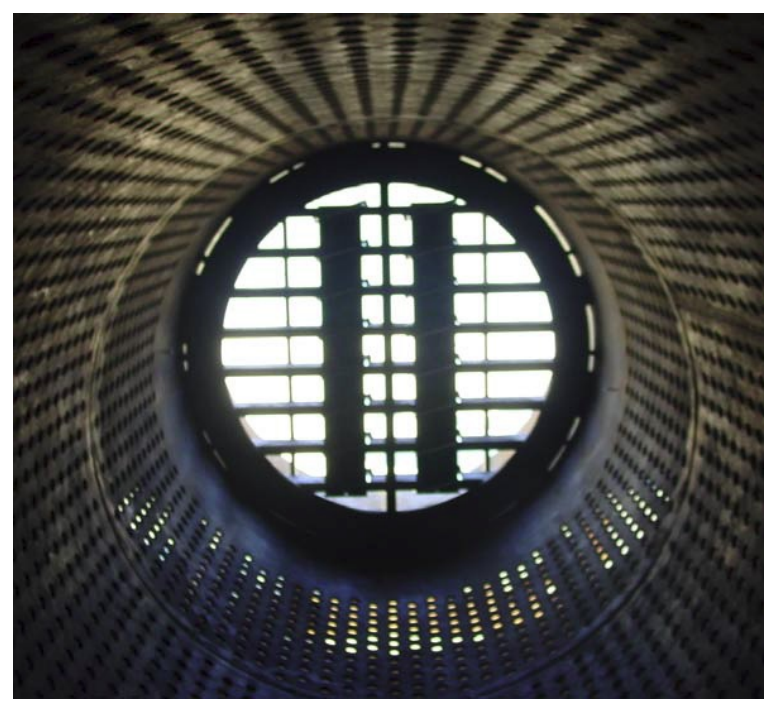

Figure 2.6: The grid termination of the ejector used by reference 44] as seen from the inside of the ejector, from [44]

ejector noise do not always follow those of the past leading to multiple authors with the same or conflicting findings. Different research purposes, techniques, geometries and conclusions are found almost on a case by case basis. There is no ejector noise literature outlining a geometry or velocity similar to the one used in this work, thus creating the need for further investigation. In addition, the noise generated by subsonic ejectors has hardly been considered. Hopefully the research outlined in this thesis can be used both for quieting of the existing configuration as well as to further the understanding of ejector noise. 


\section{Chapter 3}

\section{Potential Noise Sources, Theoretical Models and Noise Predictions}

An ejector is a fairly simple system yet there are many possible sources of noise within one. Evidently, all noise is driven by the jet issuing from the primary nozzle. Without the jet, there is no flow and thus no generated noise. The exact mechanism(s) of the noise is debated and various simplified predictions may be insightful when comparing with experimental results. This chapter provides a very brief description of a few noise generation mechanisms which seem likely in an ejector. This is followed by the model used to predict said noise and where possible, predictions are made. In maintaining with the conditions of the previous chapters, this study limits itself to the subsonic case. Furthermore, the analysis is carried out using the geometry and dimensions of the scale model used throughout this work (the scale model is discussed in the following chapters). With the exception of jet noise, only the frequency of noise is predicted, and not the sound pressure level (SPL). The resulting predictions are only estimates and the true noise within the ejector is likely to be somewhat different. 


\section{$3.1 \quad$ Jet Noise}

The jet issuing from the primary nozzle undoubtably contributes significant noise to the system. The acoustic power of a free jet is dependent on many variables including the velocity, density, temperature, and speed of sound for both the jet and ambient as well as the jet diameter, jet dynamic viscosity, and the angle of observation relative to the jet [45]. Subsonic jet noise is mainly due to turbulent mixing which takes on the form of a quadrupole ${ }^{1}$ source distribution ${ }^{2}$. This noise is mostly broadband in nature [45]. The noise is generated both from large scale coherent structures (on the order of the jet diameter) and small scale turbulence (with dimensions much smaller than the jet diameter) [46]. The noise spectrum measured perpendicular to the jet will exhibit similarity to the spectrum caused by the small scale turbulence, or Gspectrum from reference [46]. For further information on jet noise reference [46] is a highly recommended starting point.

The book Engineering Noise Control, Theory and Practice by Bies and Hansen [47] provides a simple method for the estimation of both the sound power and the $1 / 3$ octave band spectrum generated by jet noise. This practical, engineering approach of predicting jet noise only applies to air jets exhausting into air, for subsonic jets, for free-field jet noise or noise measured close to the jet, and for a Strouhal number approximation of 0.2 (based on the jet outlet diameter). While the resulting approximation may not be exact it provides an order of magnitude approximation for the noise emitted by the ejector jet. The jet overall sound pressure level, OASPL, must first be calculated using equation 3.1 ;

\footnotetext{
${ }^{1}$ The simplest noise source is a monopole, where pressure waves are distributed equally in all directions from the source, similar to a stone falling into a still pond. A dipole source is similar to two monopole sources separated by a small distance which generate pressure waves out of phase. The quadrupole source can be viewed as the superposition of two dipole sources, where the phase is dependent on the placement of the dipole sources and their respective phases

${ }^{2}$ The velocity of the jet has an impact on the source distribution but this is not discussed further
} 


$$
O A S P L=O+D I-10 \log _{10}\left(4 \pi r^{2}\right) \quad(d B, \text { re } 20 \mu P a)
$$

where $D I$ is the jet directivity index (extrapolated as $-6 \mathrm{~dB}$ at $90^{\circ}$ to the jet axis), and $r$ is the distance from the jet orifice to the point of observation. The overall sound power level, $O$, of the jet can be found using equation 3.2 .

$$
O=10 \log _{10} W_{a}+120 \quad\left(d B, \text { re } 10^{-12} \text { Watts }\right)
$$

where $W_{a}$ is the acoustical power generated by a subsonic jet in free space. It is related to the jet stream mechanical power, $W_{m}$, through an efficiency factor, $\eta$ :

$$
W_{a}=\eta W_{m}
$$

The acoustical efficiency of the jet can be approximated using equation 3.4:

$$
\eta=\left(\frac{T_{j}}{T_{\infty}}\right)^{2} \frac{\rho_{j}}{\rho_{\infty}} I M^{5}
$$

where $M$ is the jet Mach number, $T$ is the absolute temperature, $\rho$ is the density , $I$ is the acoustical power coefficient (approximately $5 * 10^{-5}$ ), the subscript ${ }_{j}$ represents the jet and the subscript $\infty$ represents ambient . In order to use this relation, $M$ must be between 0.1 and 1 , and the ratio $\frac{T_{j}}{T_{\infty}} \frac{\rho_{j}}{\rho_{\infty}}$ must be between 0.1 and 10 .

The stream mechanical power can be calculated from the jet velocity and mass flow using equation 3.5 .

$$
W_{m}=\frac{u_{j}^{2} \dot{m}_{j}}{2}
$$

Where $u_{j}$ is the jet velocity. Substituting the appropriate values into equations 3.1 . 3.5 for the design Mach number gives an OASPL of $111.4 \mathrm{~dB}$ as measured at the 
secondary flow chamber wall perpendicular to the jet axis. Given the similarity of the jet noise frequency spectrum, the sound pressure spectrum for our case can easily be predicted and it is shown in figure 3.1. The velocity of the jet efflux from the ejector is not known therefore the noise being generated by the outlet jet cannot be predicted.

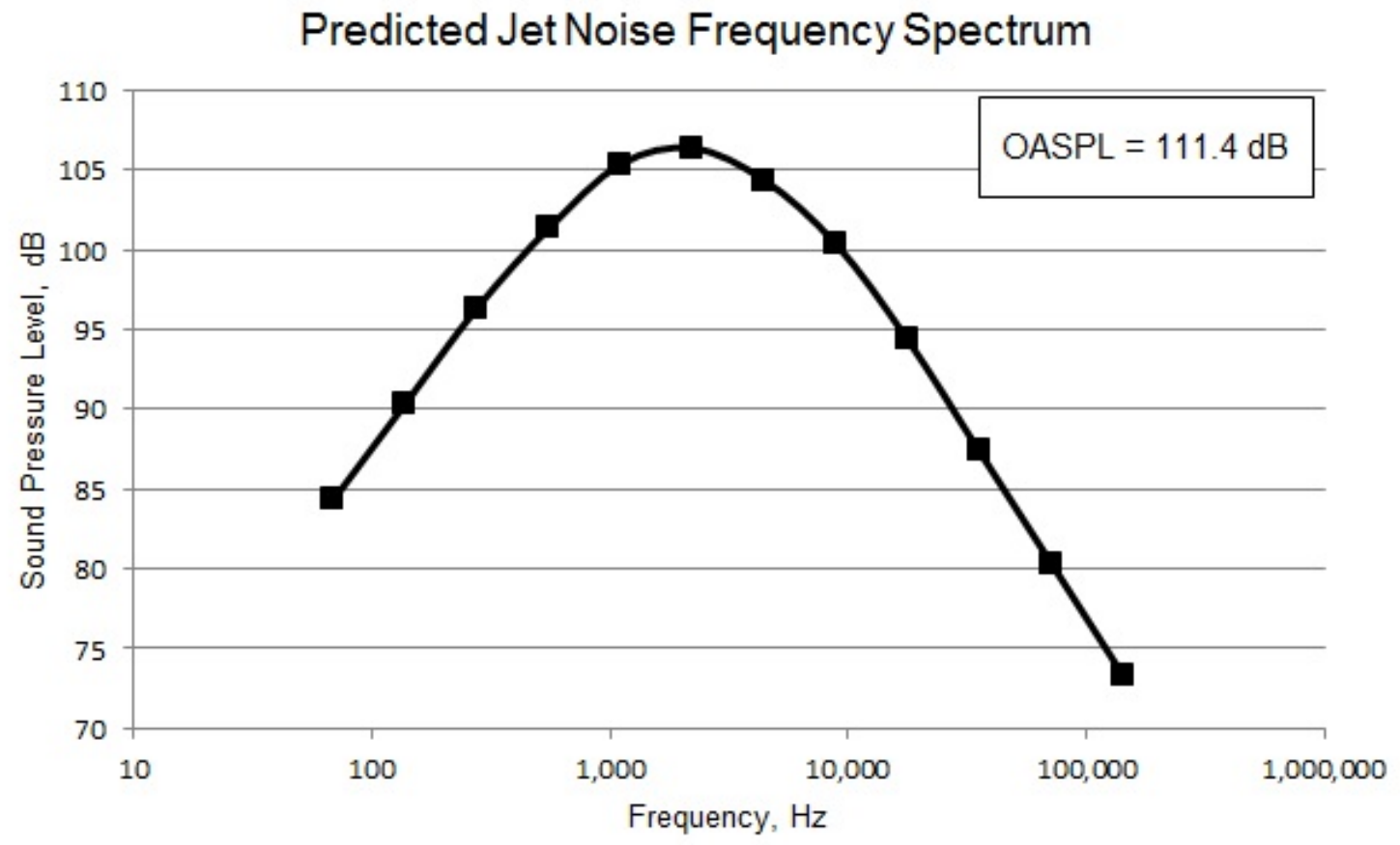

Figure 3.1: An estimate of the anticipated sound pressure spectrum caused by the scale model ejector jet

\subsection{Impingement Noise}

When a jet hits, or impinges, on a wall, noise can be generated above that found with a free jet [48. This noise can be both broadband and tonal in nature. In our ejector, the use of a silencer causes the high velocity jet to impact the cone before exiting the model. The flow fields generated by jet impingement can be surprisingly complex 
and the relationship between the flow and noise is still not well understood [49] [50].

Reference [51] outlines the mechanism of jet impingement. Similar to that found in the ejector by reference [20], a small scale instability, or coherent structure, at the nozzle outlet grows as it is convected downstream. When the structure makes contact with the wall, an acoustic disturbance in the form of a pressure wave is reflected upstream. The pressure wave reaches the nozzle shear layer at which point it triggers yet another instability thus locking the impinging jet into a feedback loop. The jet Mach number as well as the jet to wall spacing are the primary factors dictating the frequency of oscillation. Reference [52] shows a schematic of the impinging jet, recopied here in figure 3.2 .

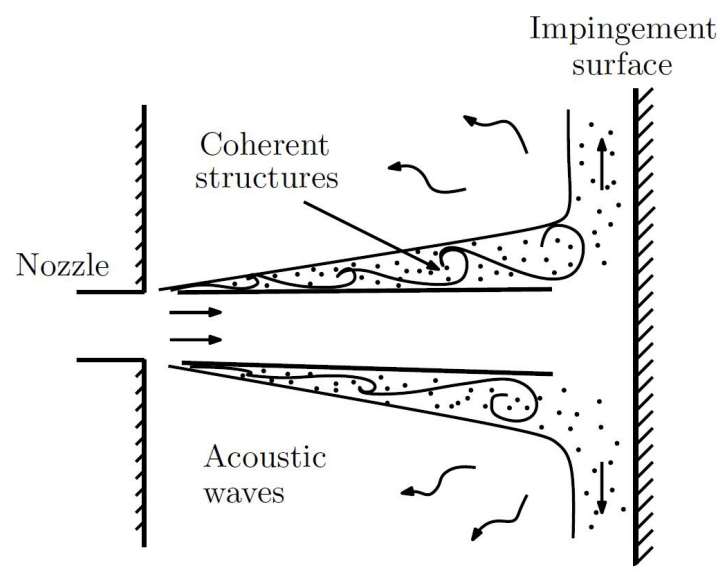

Figure 3.2: Jet impingement feedback phenomenon, adapted from [52]

The large coherent structures moving downstream take on either an axisymmetric or helical form. The axisymmetric structures can be imagined as rings wrapped around the jet which grow in size as they move downstream. The helical structures are more like a snake which winds its way around the jet. Reference [52] is a good starting point for further details on the modes. In most cases, subsonic jets only have axisymmetric oscillation, however, reference [53] finds that for small nozzle to wall distances (less than 2.3 nozzle diameters) and Mach numbers above 0.9 the 
helical oscillation is observed. The change in oscillation frequency causes a shift in the observed tone. The impingement onto a cone in the ejector may cause the jet to prefer oscillations of a helical fashion.

Reference [54] states that "in the subsonic impingement case, ... [the] Tam and Ahuja (1990) model is the only available theoretical method for frequency prediction of a self-excited subsonic impingement tone". Tam and Ahuja (1990) [55] use the formula presented by Neuwerth in 1974 [56] to predict the impingement tone frequency.

$$
f_{i m p}=\frac{n}{J\left(\frac{1}{C_{I}}+\frac{1}{C_{A}}\right)} ; \quad n=1,2,3 \ldots
$$

Where $f_{i m p}$ is the impingement frequency, $n$ is the mode of oscillation (integer), $J$ is the nozzle to wall distance, $C_{I}$ is phase velocity of the downstream propagating instability wave and $C_{A}$ is the phase velocity of the feedback acoustic waves. The phase velocity of the downstream propagating instability wave has been observed through flow visualisation and the wave properties can be found using the hydrodynamic instability wave theory. This formula assumes a negligible amount of time is taken in the reflection and excitation processes of the feedback loop.

With that being said, the use of the conventional values for these variables is likely inappropriate for estimating tones in ejectors. Firstly, the impingement in our case is onto a cone and not a flat surface. There do exist studies of this nature but they either do not focus on noise or they employ supersonic flows (see references [57 60] for example). The confinement of the flow within the ejector also imposes some differences with typical studies (which employ a free jet) and the specifics of these differences are unknown. The effective impingement length is also unknown. The exact region at which the instability waves are generated would need to be 
assumed. All in all, many assumptions would need to be made in order to predict an impingement tone and the author believes the result would likely be flawed for

this reason. Reference [61] undertakes an analysis of this type in order to have a tool to explain tones in an open jet wind tunnel. Some empirical corrections are required in order for the model to match the experimental tones. This may be fine as a tool for predicting tones at different wind tunnel operating conditions, however, a manipulation of this type is deemed inappropriate as a purely predictive tool for ejectors. In the case that flow visualization techniques could be employed in the scale model, it would allow for all the previously mentioned assumptions to be replaced with observed values, thus allowing for the calculation of an impingement tone.

\subsection{Edgetone Noise}

Edgetones have been extensively studied and have a similar behaviour to impingement tones. An edgetone is generated when a jet of air impinges on a wedge, as opposed to a wall such as in the impingement tone. A typical wedge used in edgetone studies is approximately $25^{\circ}$ but edgetones can also be generated by flow impacting any sharp edge such as a conical probe or a flat plat parallel to the flow. Given the right conditions, edgetone flows can cause acoustic tones. For brevity, the feedback mechanism of the edgetone can be assumed to the same as that of the impingement tone (details on the exact mechanism can be found in reference [62]) but the edgetone oscillates in a flapping manner - much like a flag in the wind - about the apex of the wedge. As the jet velocity or the nozzle to wedge distance changes the frequency of the tone changes gradually until the oscillation abruptly changes modes in a discrete fashion. Figure 3.3 shows simulation results of the first and second modes of oscillation (fig. 3.3 a and b respectively). Each mode will oscillate at and emit a tone of different 
frequency.
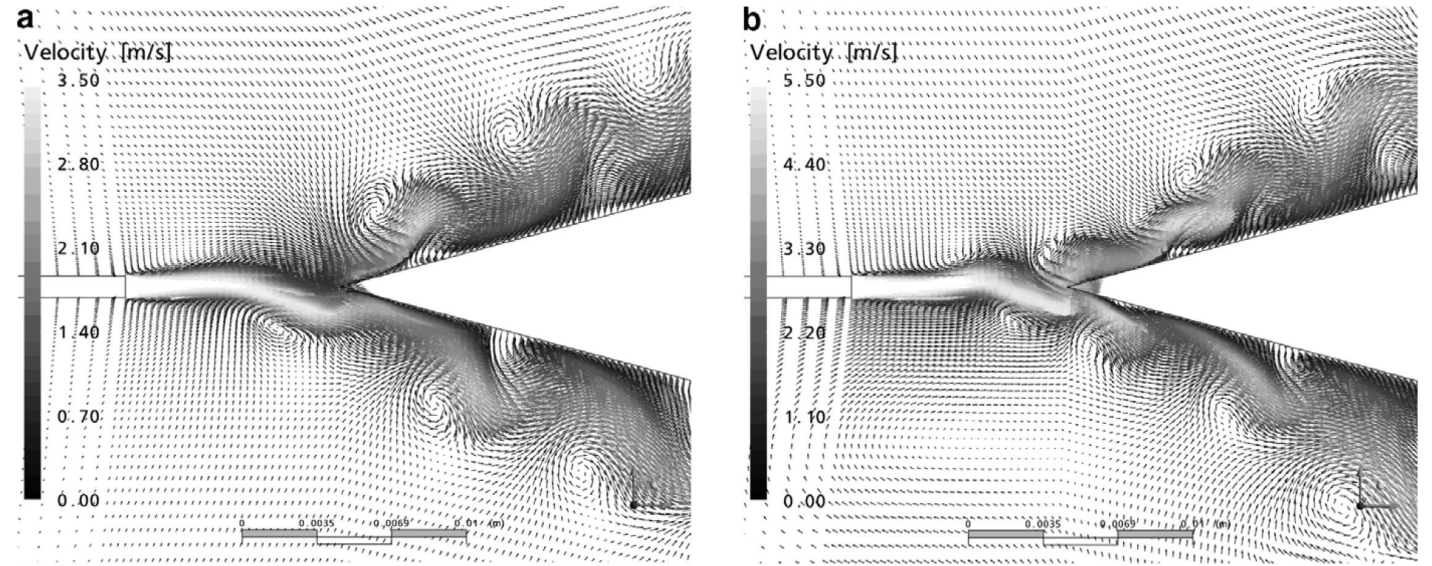

Figure 3.3: First (a) and second (b) modes of edgetone oscillation, from 62

From figure 3.3 we can see how the ejector silencer cone resembles an edgetone wedge. There are however a few important differences between typical edgetone studies and the ejector case studied here.

- Edge tone studies typically make use of a planar nozzle (rectangular cross section with a high aspect ratio) 63

- Most studies use low speed flows (low Reynolds numbers)

- The majority of studies are conducted using a wedge, not a cone

Despite these differences, there are edgetone studies in axisymmetric jets which use a conical surface as opposed to a wedge (see reference [64 for example). In addition, reference 65] studies the edge tone in flow speeds up to Mach 1 and finds that the tones persist even at very high Mach numbers.

Reference [62] provides a formula for determining the frequency of edge tones:

$$
f_{\text {edge }}=\frac{C_{e} u_{j}}{h^{q}}
$$


Where $f_{\text {edge }}$ is the frequency of oscillation, $u_{j}$ is the jet velocity, $h$ is the distance between the jet and the edge, $C_{e}$ is a constant and $q$ is also a constant whose value varies between 1 and 3/2 depending on the source. While this formula presents the general trend of edge tones, some other sources in the literature present additional constants applied to $u_{j}$ and/or $h$ in order for the formulation to more closely match experiment. The value of $q$ is not well accepted and has been the subject of a long debate $([62,66])$. The exact value appears to vary with experiment specifics (such as wedge angle or jet velocity profile). Supposing a single value for this constant becomes accepted, it remains unclear if it would be valid for use in the high speed, axisymmetric nozzle cone edgetone case.

In formula 3.7 the exit velocity profile, shape of the nozzle, wedge tip radius and transverse position of the wedge are not considered. Reference [62] explains that while these will affect the flow, they are secondary factors which have a much smaller effect than the variables in formula 3.7. To further elaborate on this, reference [67] finds that the wedge angle has little impact under $40^{\circ}$ and reference [63] experiences tones for all wedge angles investigated $\left(10^{\circ}, 20^{\circ}, 60^{\circ}\right.$ and $\left.180^{\circ}\right)$.

A further complication arises in that formula 3.7 is not the only formula used for edge tone predictions. There are many others used (see reference [66] for a good summary), some of which include empirically derived constants derived from low speed flows planar nozzle flow onto wedges. The ejector's confined jet and perforated cylinder are likely to have a significant impact on the flow and thus on the formulae. Much like in the impingement tone case, it is deemed inappropriate to attempt to predict edgetone frequencies, mostly for the same reasons. With that being said, it may be interesting to attempt to match the edgetone formula to experimental results in an attempt to explain unattributed peaks in the frequency spectrum. 


\subsection{Diffuser Noise}

The diffuser used in the ejector is an important component as it serves to slow the fluid jet resulting in less acoustic power. A diffuser is among the simplest devices in aerodynamics, however, their perceived simplicity can be misleading. Reference 68] supports this with a summary of work related to the studies on 2 conical diffusers by a research leader, 8 Ph.D students and 12 masters students which spans 29 years. Reference [69] explains that diffusers often require maximum pressure recovery in the shortest distance possible thus leading to diffusers operating near stalled conditions. These conditions may lead to transitory stall - that is a diffuser which alternates between fully stalled and not at all stalled (see figure 3.4 ) - which causes excessive losses and loud audible noise. Unfortunately, diffuser generated noise has not been studied as extensively as the previous noise sources and the mechanism of noise generation is not clear at this time. Despite this, 69] proposes that the noise is generated by a coupling of the jet preferred frequency with the natural modes of the diffuser. .
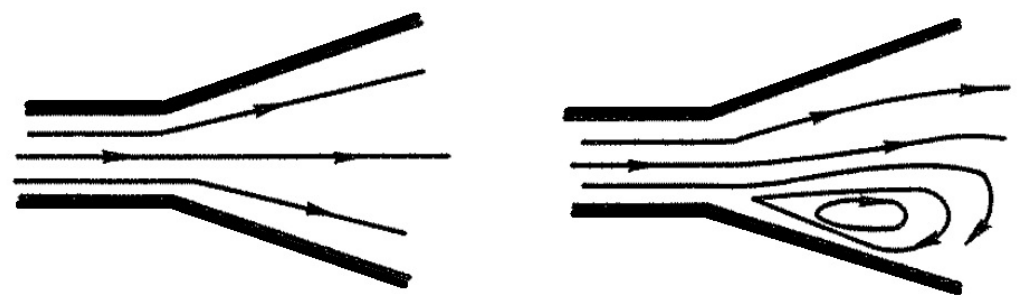

Figure 3.4: An un-stalled diffuser (left) and a fully stalled diffuser (right), adapted from 70 .

Reference [71] examines wall pressure fluctuations of low frequency in a wind tunnel diffuser. Testing of the wind tunnel with and without the diffuser causes a significant difference in the tunnel acoustics, with the difference being low frequency

\footnotetext{
${ }^{3}$ In reality these two identical diffusers may not show this drastically different performance. Simplified schematic only used for explanation
} 
tonal peaks. Reference [72] investigates transitory stall in diffusers and finds that the diffuser performance is linked to the connected ductwork. The frequencies related to the 3.75 inch $(95 \mathrm{~mm})$ inlet diffuser are found to be in the 2-3.2 $\mathrm{Hz}$ range. There are other references $\left(\left[\begin{array}{ll}73 & 76\end{array}\right)\right.$ which study diffuser generated noise but the conditions in these experiments are far from those tested in this thesis and do not apply. The takeaway is that diffuser noise can be a significant issue given the right conditions.

Reference [69] does not provide a formula for the prediction of the diffuser tones, however, these are said to be caused by the diffuser fundamental plane wave natural mode. This natural mode can be estimated using conical frustum pipe frequency predictions (can be found in reference [77] for example $]^{4}$. The natural frequency is related to the length of the diffuser wall, and can be approximated using:

$$
f_{\text {diff }}=\frac{n c}{2 \beta}
$$

where $f_{\text {diff }}$ is the frequency of oscillation, $n$ is the mode of oscillation (integer), $c$ is the speed of sound and $\beta$ is the length of the diffuser wall, as shown in figure 3.5 . This formulation assumes atmospheric pressure at both ends of the diffuser, therefore, the estimated static pressure difference from the front to the rear of the diffuser is not accounted for.

The resulting diffuser modes for the scale model are estimated to be 1760,3520 , 5280 and $7039 \mathrm{~Hz}$ for the first, second, third and fourth natural modes respectively. If a half wave exists, it would oscillate at $880 \mathrm{~Hz}$. This prediction assumes no flow, no end corrections and no relationship to other sections (the throat for example) thus caution in using these results is required as the experimental frequencies mary vary.

The work in reference [72] does provide a formula for predicting the frequency

\footnotetext{
${ }^{4}$ See also https://www.music.mcgill.ca/ gary/618/week10/node6.html
} 


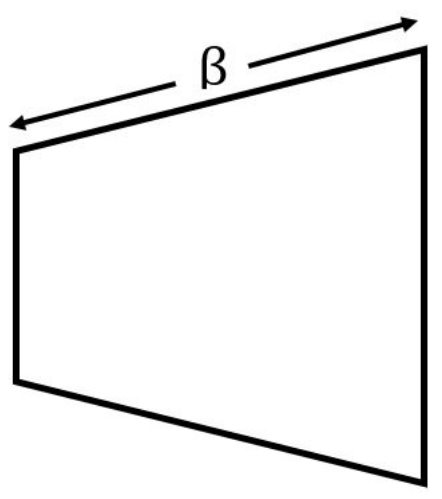

Figure 3.5: The wall length of a conical frustum for use in equation 3.8

generated, however, the frequency reported is much lower than that found by reference [69] and the mechanism cannot be the same. In addition, the formula assumes an incompressible flow which is not the case in this work given the ejector employs velocities up to sonic. Using the scaling laws presented by reference [78] (see equation 5.4), the tone from reference [72] would approximately scale to $3.5 \mathrm{~Hz}$ (well below what is recordable with the microphones used in this work) thus the mechanism is likely different.

\subsection{Acoustic Modes}

Acoustic modes - often referred to as duct modes, room resonance, resonant modes, standing waves, duct acoustics, eigenfrequencies, natural frequencies or natural modes to name a few - are frequencies at which an enclosure is most susceptible to acoustic excitation. This is analogous to a mechanical system which will undergo large oscillations if excited at its natural frequency. The frequencies of oscillation are functions of the size and shape of a given enclosure and are easy to predict for simple enclosure geometries.

Seemingly the simplest example of an acoustic natural mode is the excitation of an 
air gap between two parallel walls. If a source of excitation has a frequency matching the natural frequency of the air gap, the molecules of air within the gap will begin to oscillate with large amplitude. The molecules at the walls are confined thus cannot move but the molecules in the middle of the gap are free and they oscillate from wall to wall with a large amplitude. The resulting molecular velocity will be zero at the walls and highest in the center of the gap. The pressure will vary between minimum and maximum at the walls, depending on the time of observation. Conversely, in the center of the gap where there is a large velocity the gage pressure is zero. The regions with no velocity or pressure are known as nodes (velocity node and pressure node respectively) and the regions of maximum velocity or pressure are known as antinodes.

Fluids flow from high pressure regions to low pressure regions thus the high pressure region generated near one of the walls will begin to move towards the low pressure region at the other wall. This happens back and forth, and resonance occurs. This process is shown in figure 3.6 where the vertical lines represent the relative pressure of the air. The case described corresponds to the fundamental resonance where the wavelength of the fluctuating pressure is double the length of the wall gap. This 1-dimensional case is often called axial resonance ([1,0,0] or $[0,1,0]$ for example).

If the excitation frequency is doubled the oscillation will take on a second form, known as the second harmonic, or mode, where the very center of the air gap becomes a velocity node. The wavelength will be half as long thus the frequency will be double. Higher order harmonics also exist, occurring in integers of the fundamental. In the case where the air gap becomes enclosed by walls on four sides, the same process can occur in the newly enclosed direction. This can happen independently or in tandem with oscillations in the first direction. When occurring in tandem $([1,1,0]$ 


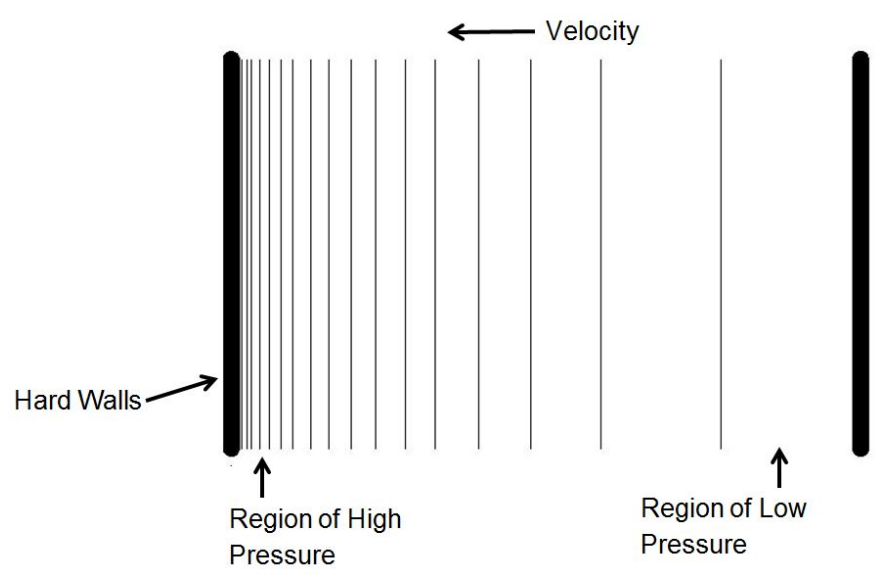

Figure 3.6: Example of a pressure oscillation between two walls

or $[0,1,1]$ or $[2,0,1]$ for example), this is referred to as a tangential oscillation which has approximately half the power of axial oscillations. Adding walls to the third direction also causes an oscillation in the third direction to be possible independently or in tandem with the two other dimensions. When oscillations occur in tandem in all three dimensions $([1,1,1]$ or $[2,2,2]$ for example), this oscillation is referred to as oblique and has approximately a quarter of the power of axial oscillations.

The frequency of oscillation between two surfaces can be found using equation 3.9

$$
f_{\text {anm }}=\frac{n c}{2 L}
$$

where $n$ is the mode of oscillation $(1,2,3 \ldots)$ and $L$ is the distance between the two walls. It is important to note that this formula does not take into consideration the acoustic reflection properties of the walls (or of air absorption for that matter). This formula also lacks a time component, thus the way sound may be reflected and dissipated with time is unaccounted for. Despite this, it is a valuable simplified tool used for predicting the frequencies encountered. This relationship can be extended to three dimensions as follows: 


$$
f_{\text {anm }}=\frac{c}{2} \sqrt{\left(\frac{n_{x}}{L_{x}}\right)^{2}+\left(\frac{n_{y}}{L_{y}}\right)^{2}+\left(\frac{n_{z}}{L_{z}}\right)^{2}}
$$

where $n_{x}, n_{y}$ and $n_{z}$ are the modes of oscillation $(0,1,2,3 \ldots)$ in the $x, y$ and $z$ directions respectively and $L_{x}, L_{y}$, and $L_{z}$ are the dimensions of the enclosure in the $x, y$ and $z$ directions respectively. This formula allows for the calculation of the acoustics of an enclosure simultaneously oscillating in combined directions. The resonance is often described using the mode numbers enclosed in square brackets in the form $\left[n_{x}, n_{y}, n_{z}\right]$ (for example $[1,0,1]$ is a fundamental resonance in the $x$ and $z$ directions), as seen previously.

The acoustic resonance of an enclosure changes drastically when removing one of the 6 walls. When this occurs, such as in the secondary flow chamber and outlet chamber, the removed wall no longer causes a velocity node but rather a velocity antinode is established. This has the effect of changing the fundamental resonance from a half wavelength to a quarter wavelength. The longitudinal frequency calculation thus becomes:

$$
f_{\text {anm }}=\frac{n c}{4 L}
$$

The direction of oscillation in rooms is simply labelled as the $\mathrm{x}, \mathrm{y}$ and $\mathrm{z}$ direction of oscillation, however, ducts or pipes (such as the throat of the ejector) use a different nomenclature. Oscillation along the axis of the pipe is referred to as longitudinal and from wall to wall of the pipe is known as the transverse mode (sometimes the cross or radial modes). A third common mode, known as the azimuthal mode, happens when the fluid oscillates in a rotational fashion with the center of rotation being the axis of symmetry of the pipe. Figure 3.7 shows a schematic of the fluid displacement for these three modes. 

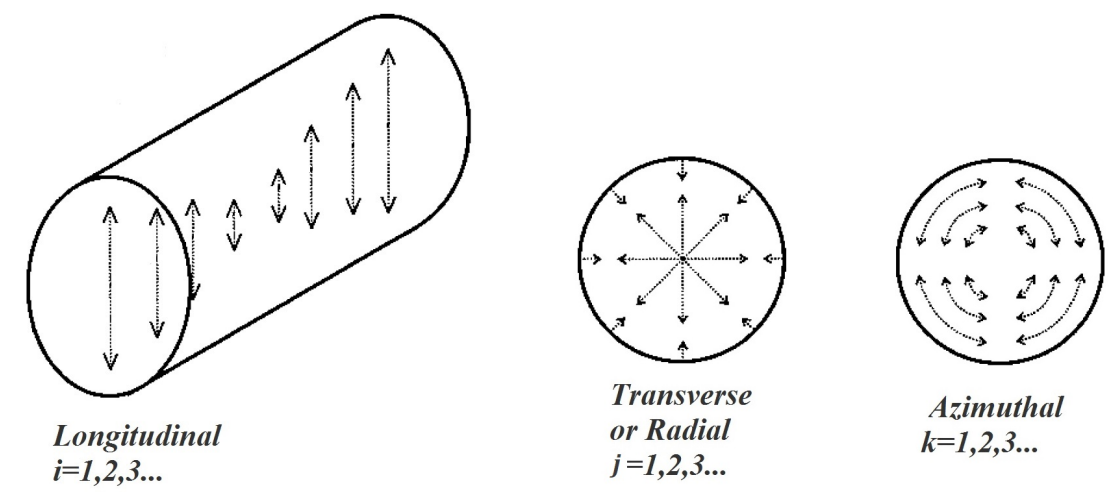

Figure 3.7: Longitudinal, azimuthal and transverse oscillation in a duct, adapted from [79]

The acoustic modes for a pipe with both ends open can be calculated from equation 3.12 :

$$
f_{i, j, k}=\left[\sigma_{j k}^{2}+\left(i \frac{\pi d_{d u c t}}{2 l}\right)^{2}\right]^{\frac{1}{2}} \frac{c}{\pi D}
$$

where $\sigma_{j k}$ is the $j^{t h}, k^{t h}$ root of the Bessel function derivative $\biguplus^{5} i, j$ and $k$ are the longitudinal, transverse and azimuthal mode of oscillation $(0,1,2,3 \ldots)$ respectively, $d_{d u c t}$ is the duct diameter and $L$ is the duct length. In practice it has been found that the longitudinal modes of oscillation for a pipe of a given length occur at a frequency corresponding to that of a slightly longer pipe. An end correction is applied to account for this difference. An exact correction value is not agreed upon in literature and for this work an end correction of 0.8 duct diameters is used. That is, the effective length, $L_{\text {eff }}$ to be used in equation 3.12 is:

$$
l_{\text {eff }}=l+0.8 d_{d u c t}
$$

For the case of a pipe with one end open, equation 3.11 can be used to calculate

\footnotetext{
${ }^{5}$ see Appendix B for a table of the Bessel function derivative roots
} 
the longitudinal frequencies. Likewise, the case of longitudinal modes in a pipe with both ends closed reduces to equation 3.9. With the equations presented, predictions on the resonance modes of the ejector can be made.

\subsubsection{Acoustic Mode Prediction for the Secondary Flow Chamber}

In order to estimate the modes in the secondary flow chamber, the chamber is represented by a simple box with one end open and no primary nozzle. Based on the dimensions of the secondary flow chamber, the acoustic modes are calculated and presented in table 3.1 and 3.2 . For brevity, only the fundamental combinations and first 3 octaves of individual modes are shown. The $x$ and $z$ directions are used for the width and height of the chamber (equal dimensions) whereas the $y$ direction represents the length of the chamber.

Table 3.1: Fundamental Modes of the Secondary Flow Chamber

\begin{tabular}{cccc}
\multicolumn{3}{c}{ Modes } & Frequency, Hz \\
\hline$n_{x}$ & $n_{y}$ & $n_{z}$ & \\
1 & 0 & 0 & 895 \\
0 & 1 & 0 & 146 \\
0 & 0 & 1 & 895 \\
1 & 1 & 0 & 906 \\
1 & 0 & 1 & 1264 \\
0 & 1 & 1 & 906 \\
1 & 1 & 1 & 1273
\end{tabular}


Table 3.2: Octaves of the Fundamental Modes of the Secondary Flow Chamber

\begin{tabular}{cccc} 
& \multicolumn{3}{c}{ Frequency, $\mathrm{Hz}$} \\
Octave & 1 & 2 & 3 \\
\hline$n_{x}$ & 895 & 1788 & 2682 \\
$n_{y}$ & 146 & $\mathrm{~N} / \mathrm{A}^{6}$ & 439 \\
$n_{z}$ & 895 & 1788 & 2682
\end{tabular}

\subsubsection{Acoustic Mode Prediction for the Outlet Chamber}

In order to direct the flow in the scale model, an outlet flow chamber is required (details in chapter 4). In essence, this is a 5 sided box directing the flow from the silencer to a given direction. The outlet flow chamber acoustic modes are estimated in the same manner as for the secondary flow chamber with the results presented in table 3.3 and 3.4 . The $x$ dimension is taken as perpendicular to the primary jet axis, the $y$ dimension as parallel to the primary jet axis and the $z$ direction as the longest dimension.

Table 3.3: Fundamental Modes of the Outlet Flow Chamber

\begin{tabular}{cccc}
\multicolumn{3}{c}{ Modes } & Frequency, Hz \\
\hline$n_{x}$ & $n_{y}$ & $n_{z}$ & \\
1 & 0 & 0 & 894 \\
0 & 1 & 0 & 838 \\
0 & 0 & 1 & 190 \\
1 & 1 & 0 & 1225 \\
1 & 0 & 1 & 914 \\
0 & 1 & 1 & 859 \\
1 & 1 & 1 & 1240
\end{tabular}


Table 3.4: Octaves of the Fundamental Modes of the Outlet Flow Chamber

\begin{tabular}{cccc} 
& \multicolumn{3}{c}{ Frequency, $\mathrm{Hz}$} \\
Octave & 1 & 2 & 3 \\
\hline$n_{x}$ & 894 & 1788 & 2682 \\
$n_{y}$ & 838 & 1675 & 2513 \\
$n_{z}$ & 190 & $\mathrm{~N} / \mathrm{A} \bigsqcup^{7}$ & 569
\end{tabular}

\subsubsection{Acoustic Mode Prediction for the Throat}

The calculated modes of the throat are presented in tables 3.5 to 3.7 . The fundamental combinations are presented as well as the octaves of the longitudinal $(i)$, transverse $(j)$ and azimuthal $(k)$ modes. The $10^{t h}, 2^{\text {nd }}$ and $3^{\text {rd }}$ octaves (respectively) are presented as these are the modes of greatest influence 8

Table 3.5: Fundamental Modes of the Throat

\begin{tabular}{cccc}
\multicolumn{3}{c}{ Modes } & Frequency, $\mathrm{Hz}$ \\
\hline$i$ & $j$ & $k$ & \\
1 & 1 & 0 & 5397 \\
1 & 0 & 1 & 2608 \\
0 & 1 & 1 & 7497 \\
1 & 1 & 1 & 7504
\end{tabular}

\footnotetext{
${ }^{8}$ As determined by experimental tests
} 
Table 3.6: Longitudinal Modes of the Throat

\begin{tabular}{cccc}
\multicolumn{3}{c}{ Modes } & Frequency, Hz \\
\hline$i$ & $j$ & $k$ & \\
0.5 & 0 & 0 & 157 \\
1 & 0 & 0 & 314 \\
2 & 0 & 0 & 627 \\
3 & 0 & 0 & 941 \\
4 & 0 & 0 & 1255 \\
5 & 0 & 0 & 1568 \\
6 & 0 & 0 & 1882 \\
7 & 0 & 0 & 2196 \\
8 & 0 & 0 & 2509 \\
9 & 0 & 0 & 2823 \\
10 & 0 & 0 & 3137
\end{tabular}

Table 3.7: Transverse Modes of the Throat

\begin{tabular}{cccc}
\multicolumn{3}{c}{ Modes } & Frequency, $\mathrm{Hz}$ \\
\hline$i$ & $j$ & $k$ & \\
0 & 0.5 & 0 & 2694 \\
0 & 1 & 0 & 5388 \\
0 & 2 & 0 & 9865
\end{tabular}

Table 3.8: Azimuthal Modes of the Throat

\begin{tabular}{|c|c|c|}
\hline \multicolumn{2}{|c|}{ Modes } & Frequency, $\mathrm{Hz}$ \\
\hline & $j \quad l$ & \\
\hline 0 & 0 & 2589 \\
\hline 0 & 02 & 4295 \\
\hline & $0 \quad 3$ & 5908 \\
\hline
\end{tabular}




\subsubsection{Schroeder Frequency}

An interesting property pertaining to natural acoustic modes (room acoustics) is the Schroeder ${ }^{9}$ frequency, $f_{S}$. Below this crossover frequency, in multimode systems individual well-separated resonances occur whereas above the Schroeder frequency there are many overlapping normal modes. Essentially, below this frequency individual modes are strongly observed whereas above this frequency an enclosure acts as a reverberant room and the acoustics follow a statistical behaviour. In practice the transition happens over a frequency range and not a discrete value, but for simplicity this is not discussed here. The Schroeder frequency can be calculated using equation 3.14 from reference [80]:

$$
f_{S}=2000 \sqrt{\frac{t}{v_{\text {encl }}}}
$$

Where $t$ is the $60 \mathrm{~dB}$ reverberation time (in seconds) and $v_{\text {encl }}$ is the volume of the given enclosure (in cubic meters). The reverberation time can be estimated using the formula proposed by Sabine [81]:

$$
t=\frac{24 \ln 10}{c} \frac{v_{\text {encl }}}{e}
$$

Where $e$ is the product of the enclosure surface area and the absorption coefficient of the material ${ }^{10}$, The Schroeder frequencies of the secondary flow chamber, outlet flow chamber and throat can be estimated at 3504, 4957 and 12,987 $\mathrm{Hz}$ respectively. For the secondary and outlet flow chambers, this is within the range of measurement thus a change in behaviour is to be expected for higher frequencies.

\footnotetext{
${ }^{9}$ Not to be confused with Schrodinger, who had a pet cat

${ }^{10}$ See table 5.1 for the absorption coefficient values, mean value used
} 


\subsubsection{Acoustic Mode Prediction for the Entire Ejector}

A final acoustic mode worth predicting is the mode associated with the entire length of the ejector. In essence, the ejector acts as a large open-open pipe. The centerline distance from the inlet plane to the outlet plane of the ejector can be used to estimate the frequency associated with this oscillation. This mode has the longest wavelength of any in the ejector thus it is responsible for the lowest frequencies experienced by the ejector. The resulting frequency prediction and its octaves are presented in table 3.9 .

Table 3.9: Fundamental Modes of the Entire Ejector Mode of Oscillation Frequency, $\mathrm{Hz}$

\begin{tabular}{cc}
\hline 0.5 & 117 \\
1 & 234 \\
2 & 351 \\
3 & 468
\end{tabular}

\subsubsection{Prediction of the Jet Preferred Frequency}

A prediction of the preferred mode of oscillation of the jet is included in this section due to the likelihood of strong tones being generated if the jet preferred mode couples to one of the aforementioned modes. The preferred frequency depends on the jet velocity and can be calculated using the Strouhal Number, shown in equation 3.16.

$$
S t=f_{j} d_{j} / U_{j}
$$

where St is the Strouhal number, estimated as 0.3 (the same as in [32]), $f_{j}$ is the preferred frequency of oscillation of the jet, $d_{j}$ is the jet diameter and $U_{j}$ is the jet 
velocity. The preferred frequency is found to be $2327 \mathrm{~Hz}$. This does not match any of the previously predicted modes exactly, however, it is within 200-300 Hz of many modes and the Strouhal number has been found to shift when the jet frequency is near that of an acoustic mode to which it couples.

\subsection{Summary}

In summary, the jet noise is expected to be of $111 \mathrm{~dB}$ with a broadband spectrum. The jet impingement and edgetone noises are not estimated but they may cause important tones. The diffuser noise is predicted to have a full wave resonance of $1760 \mathrm{~Hz}$ and its octaves are possible, including the half wave resonance. There is a multitude of acoustic natural modes spanning a wealth of frequencies. This makes acoustic resonance likely and by recording the noise in different locations of the ejector the resonant modes of importance can likely be isolated. Finally, the jet has an estimated preferred natural frequency of oscillation of $2327 \mathrm{~Hz}$ which does not match any acoustic modes exactly but the jet frequency could easily shift to match one of the many nearby acoustic modes (coupling). 


\section{Chapter 4}

\section{Experimental Setup}

In order to experimentally investigate ejector noise, a scale model ejector is designed and built. The scale model is used in conjunction with a newly commissioned anechoic chamber and Carleton University's Pratt and Whitney High Speed Wind Tunnel. The scale model testing is conducted inside the anechoic chamber with a special arrangement to allow the blow-down air from the wind tunnel to be used as an air source providing air to the primary nozzle. Details of these components as well as the control system used to control the wind tunnel are presented in this chapter along with the instrumentation used and measurement uncertainties.

\subsection{Scale Model}

The use of a scale model for acoustic investigations is opted for given the large computational costs of computational aeroacoustics and the existing facilities at Carleton University. The wide range of applications for ejectors leads to a variety of possible scale model geometries. For this work the scale model is geometrically similar to a large ejector ${ }^{1}$.

\footnotetext{
${ }^{1}$ Exact application omitted to protect intellectual property
} 
The scale model ejector is shown schematically in figure 4.1 with the relevant components listed. The scale model consists of: an inlet perforated plate (flow conditioner) (item 1), the primary nozzle (item 2), the inlet convergence (item 3), the throat (item 4), the diffuser (item 5), the silencer assembly (item 6), an outlet chamber perforated plate (flow conditioner) (item 7), a base assembly (item 8), the outlet flow chamber (item 9) and knobs for easy scale model disassembly (item 10). The exterior of the scale model is covered with an acoustic foam to reduce reflections and sound transmission through the model walls.
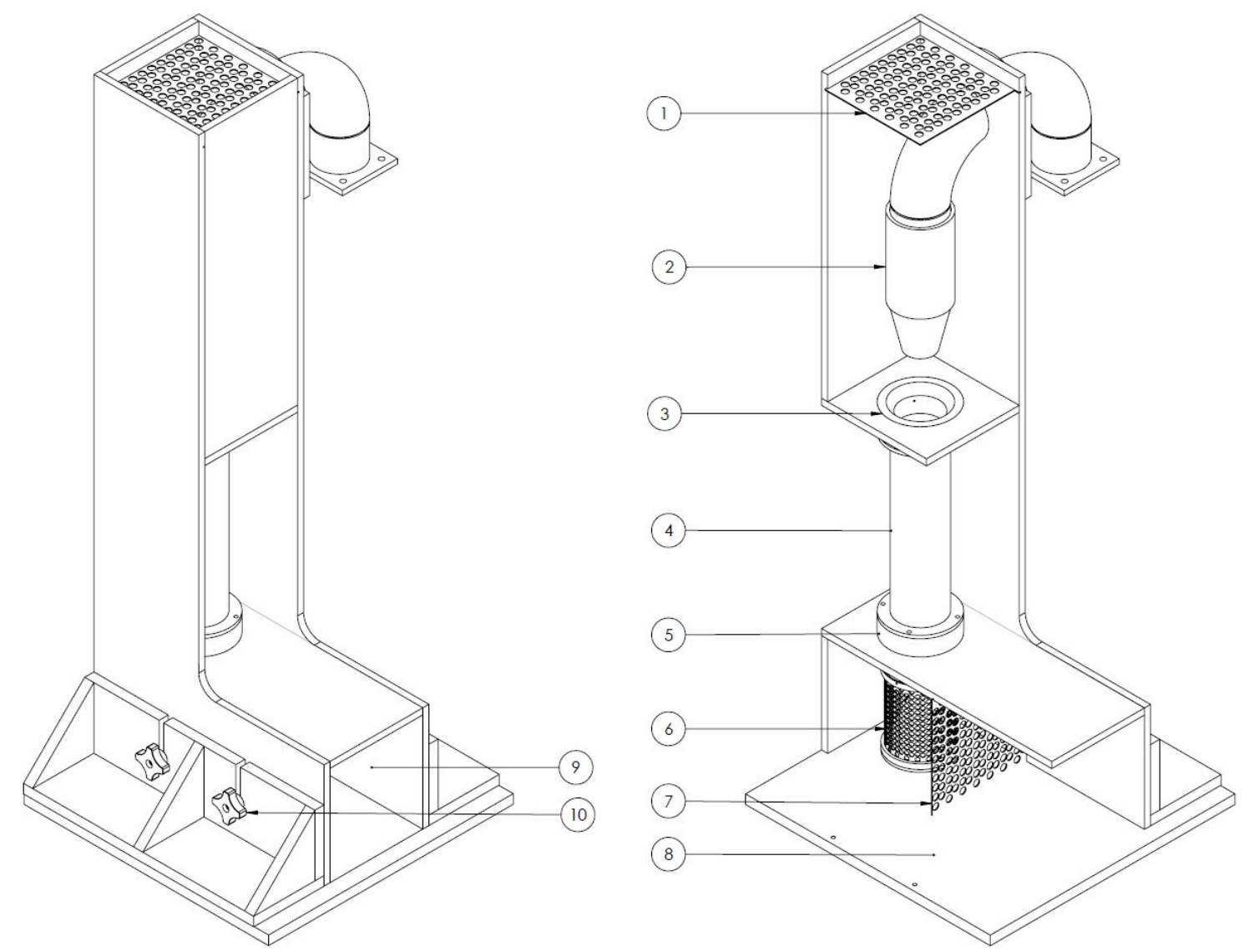

Figure 4.1: A schematic of the scale model (left) and the same schematic with components hidden (right) to allow the internal components to be visualized

The primary nozzle contraction is $26^{\circ}$ with a length of 2.5 primary nozzle diameters 
and it is 2.5 nozzle diameters from the inlet convergence. The secondary flow chamber is roughly $7.5 \times 7.5 \times 15.5$ nozzle diameters with a flow path concentric to the primary nozzle. The inlet convergence has an area ratio of 1.7 with an opening size of 2.5 nozzle diameters and length of 0.5 nozzle diameters. The throat is 2 nozzle diameters in diameter and 9 nozzle diameters long. The diffuser is 2.5 nozzle diameters long with an area ratio of $1.55\left(7.5^{\circ}\right.$ half angle). The perforated cylinder is 2.75 nozzle diameters in diameter and 3.5 nozzle diameters long. The cone is 45 degrees and of the same base diameter as the perforated cylinder. The outlet flow chamber is 5 nozzle diameters wide (perpendicular to throat), 5.5 deep (parallel to throat) and 12 high. The outlet perforated plate is 6 nozzle diameters from the wall to which it is parallel: $:^{2}$

During operation, the air from the blowdown tanks is routed to the primary nozzle. Upon entering the primary nozzle, the air turns 180 degrees and passes through flow conditioning devices. It then encounters a converging nozzle which accelerates it to the desired speed. The air jet issuing from the primary nozzle contraction, inside the secondary flow chamber, entrains the surrounding air which flows through the scale model. There is a perforated plate at the inlet of the scale model to provide a slight pressure drop to the entrained air as well as to act as a flow conditioner. Likewise, the outlet chamber contains a perforated plate which provides a pressure drop and provides some flow uniformity to the air leaving the scale model.

Upon reaching the scale model outlet the air flows into the anechoic chamber collector assembly and out of the anechoic chamber. The collector cross sectional area is roughly half that of the scale model outlet area and the collector is placed roughly an inch $(2.5 \mathrm{~cm})$ downstream of the scale model outlet due to space constraints, therefore some of the outlet air inevitably impinges on the collector and a good portion of this

\footnotetext{
${ }^{2}$ All values rounded to nearest 0.25
} 
low-velocity air is recirculated inside the anechoic chamber.

The scale model is placed vertically inside the anechoic chamber in order to use the greatest possible amount of space inside the chamber. The primary nozzle is contained within a box enclosed on 5 sides, similar to the "intake box" used by Middleton [19], for the same reason as Middleton. At the outlet of the ejector, the silencer is housed inside an outlet chamber in order to permit testing in the anechoic chamber. The outlet chamber serves to redirect the air which has passed through the silencer into the collector and out of the chamber. Without this outlet chamber the flow could impinge on the wedges destroying them or cause an unacceptable buildup of pressure inside the anechoic chamber. Further details on each individual component of the scale model are presented in the following chapter.

\subsection{Anechoic Chamber}

The anechoic chamber used in this work was designed and commissioned by Giardino [82] in 2018. An overview is presented here, however, for full details see the work of Giardino. In its nominal configuration, the chamber is used for "flat plate" type tests where an inlet nozzle penetrates a chamber wall providing a flow over the flat plate. The space surrounding the nozzle penetration is acoustically sealed to prevent noise contamination inside the chamber. The flow is then captured by a collector before being diffused, turned 90 degrees upwards, diffused a second time, and exhausted to atmosphere. Figure 4.2 shows the front, back and partial cutaway views of the chamber as used in its nominal configuration.

The chamber is made of 4 inch $(102 \mathrm{~mm})$ thick acoustic panelled walls and is lined with perforated sheet metal wedges. The wedges are sized such that the chamber has a $250 \mathrm{~Hz}$ cut off frequency. The floor wedges are removable allowing access to the test 


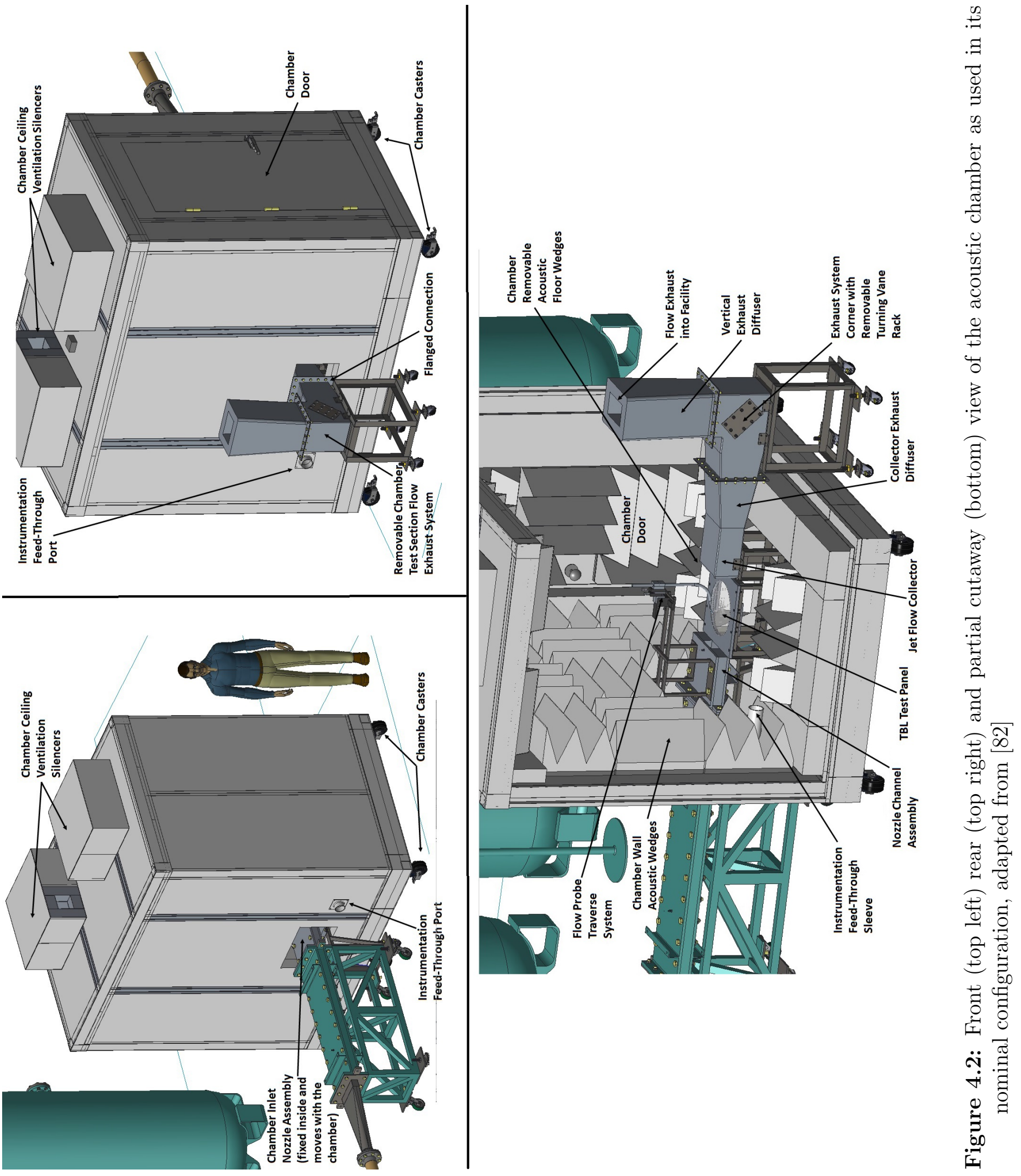




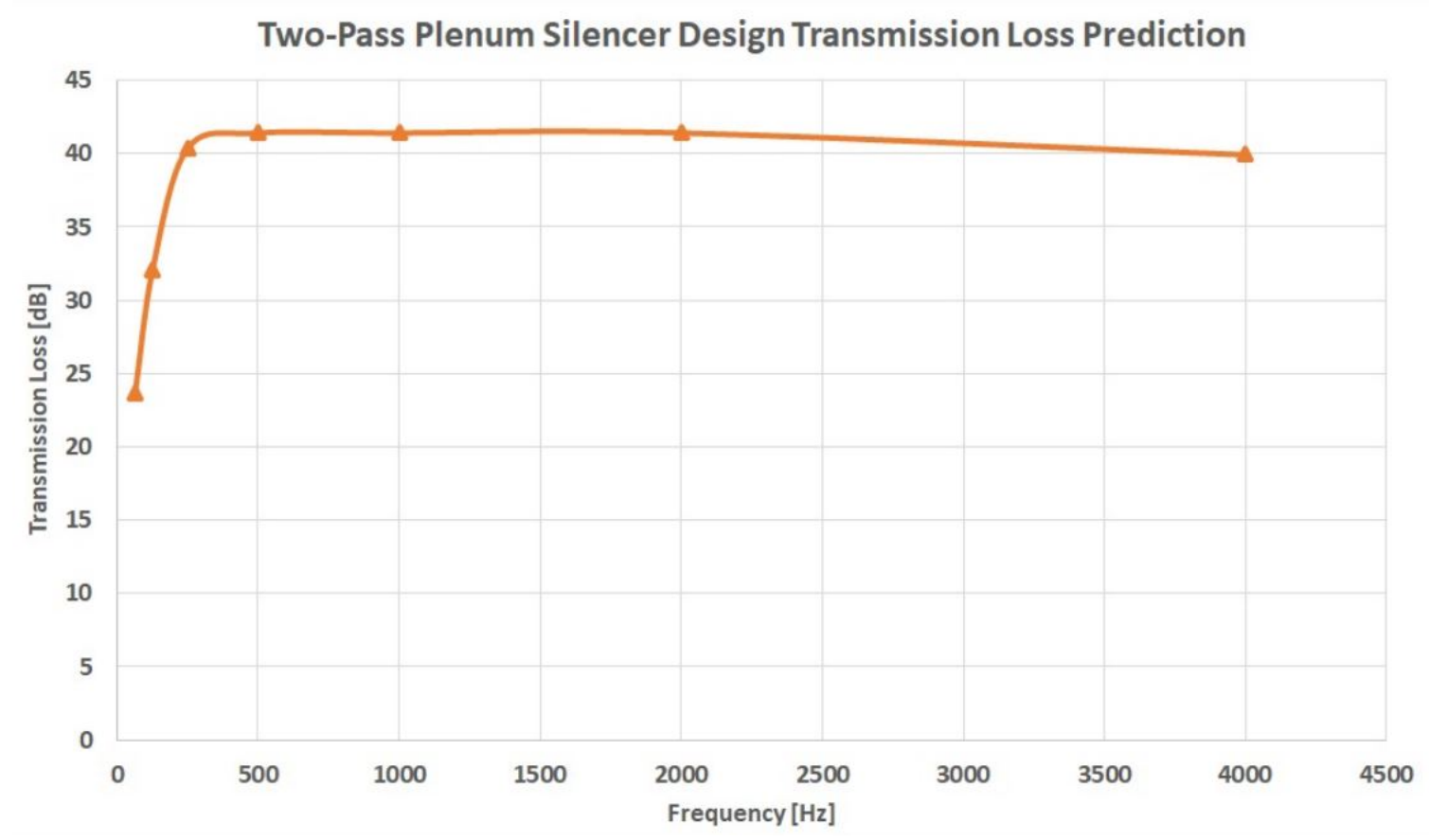

Figure 4.3: The predicted transmission loss for the anechoic chamber silencers, adapted from [82]

section. The roof of the chamber contains two roughly 6 inch $(152 \mathrm{~mm})$ diameter holes which are capped with silencers to allow the chamber pressure to equalize with the outside atmosphere while preventing noise contamination inside the chamber. The silencer performance has not yet been characterized, however, figure 4.3 provides the transmission loss estimated by Giardino.

What is termed as the collector assembly, shown in figure 4.4, is composed of a jet collector, diffuser, corner with turning vanes, and a secondary diffuser (in the order encountered by the flow). The collector has a smooth interior which converges in such a manner to avoid flow separation in the nominal configuration while still catching the jet potential core and shear layers. Following this initial contraction is a 0.56 meter (22 inch) diffuser with an outlet to inlet area ratio of 2 and a divergence angle of $7.7^{\circ}$. Downstream of this diffuser is a $90^{\circ}$ corner fit with sheet metal turning vanes 


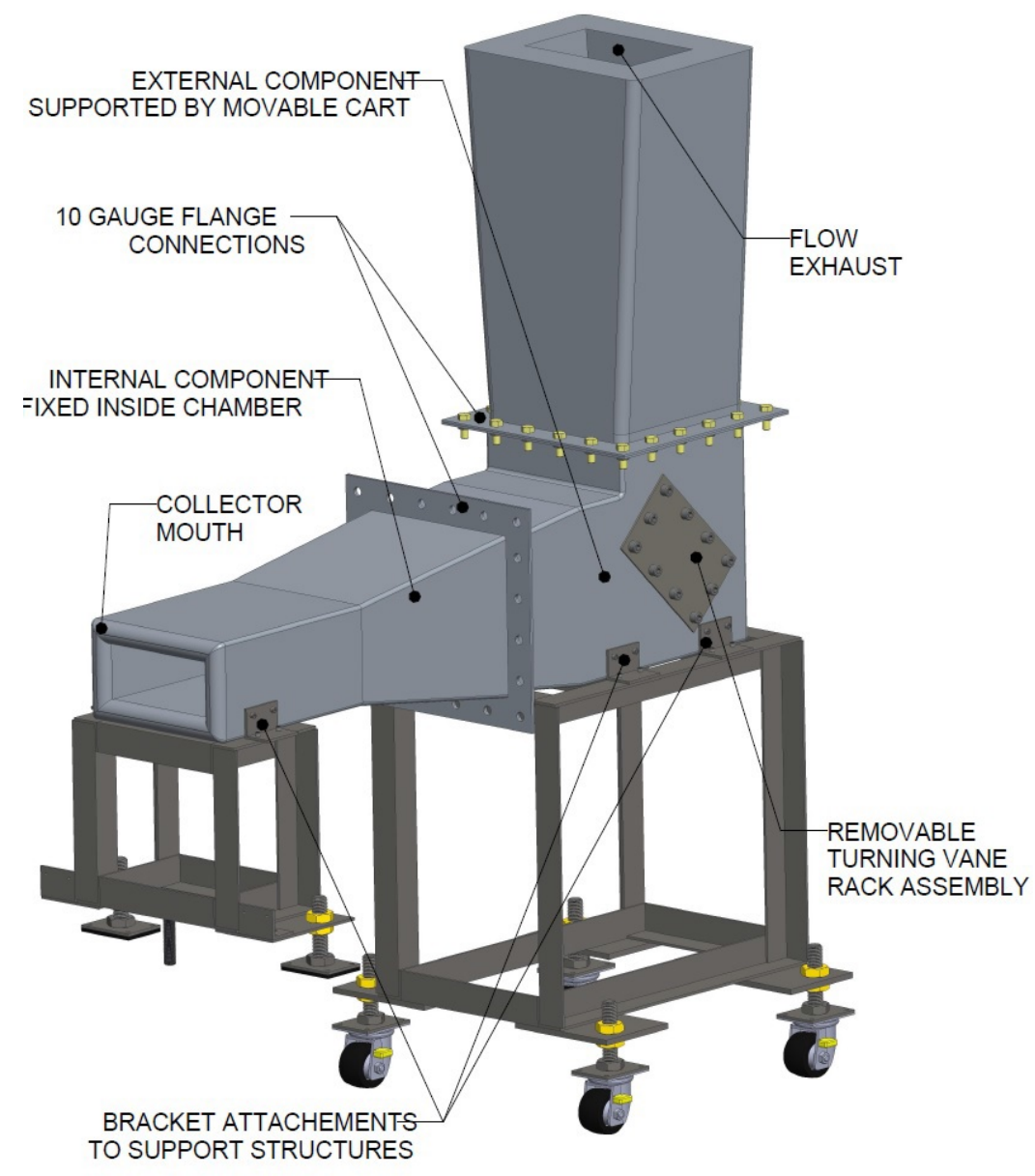

Figure 4.4: Labelled 3D CAD rendering of the collector assembly, from [82]

which themselves precede a second vertical diffuser. This diffuser is 0.61 meters (24 inches) long with an outlet to inlet area ratio of 1.56 and a divergence angle of $8^{\circ}$. Everything downstream of the initial collector contraction is made of perforated sheet metal backed by acoustic insulation in order to help attenuate noise propagation.

In order to perform tests on the ejector scale model some reconfiguration of the chamber is required. Firstly, the nozzle is disconnected, blocked and acoustically treated to prevent noise contamination from the facility leaking into the chamber. The flat plate is removed and replaced with the scale model base assembly upon which the scale model is mounted (component 8 from figure 4.1, see figure 5.6). The 
collector is kept as is and a portion of the flow from the scale model exhausts into the collector. Finally, an access panel to the side of the collector is modified in order to allow for a flexible air hose to be fed to the scale model while preventing noise contamination through the opening. Only with these temporary modifications made are scale model tests possible. Figure 4.5 shows a photo of the scale model mounted in the anechoic chamber (without the flexible hose connected) and figure 4.6 is a photo showing the modified access panel for hose access with the external portion of the collector assembly behind it.

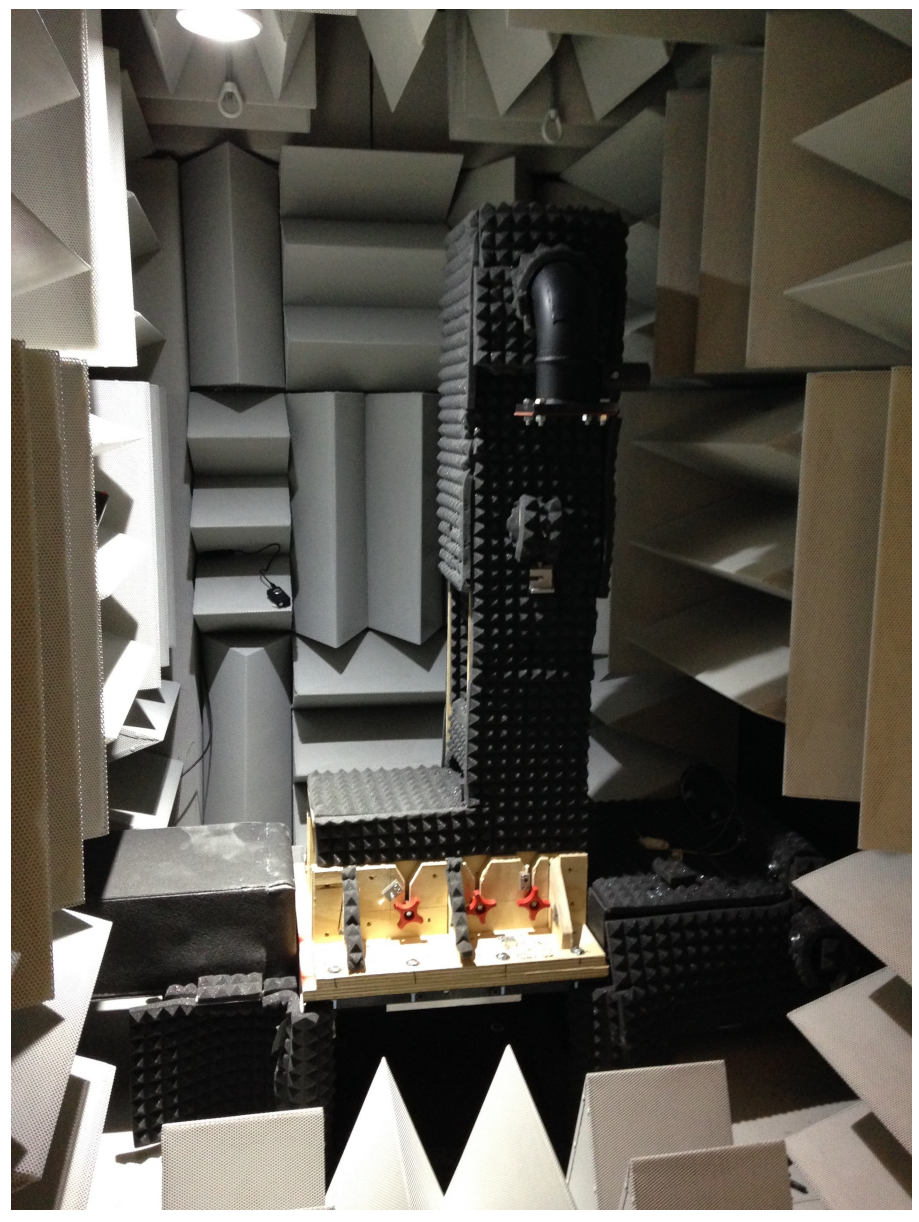

Figure 4.5: The scale model mounted in the anechoic chamber, pictured without the flexible air hose attached 


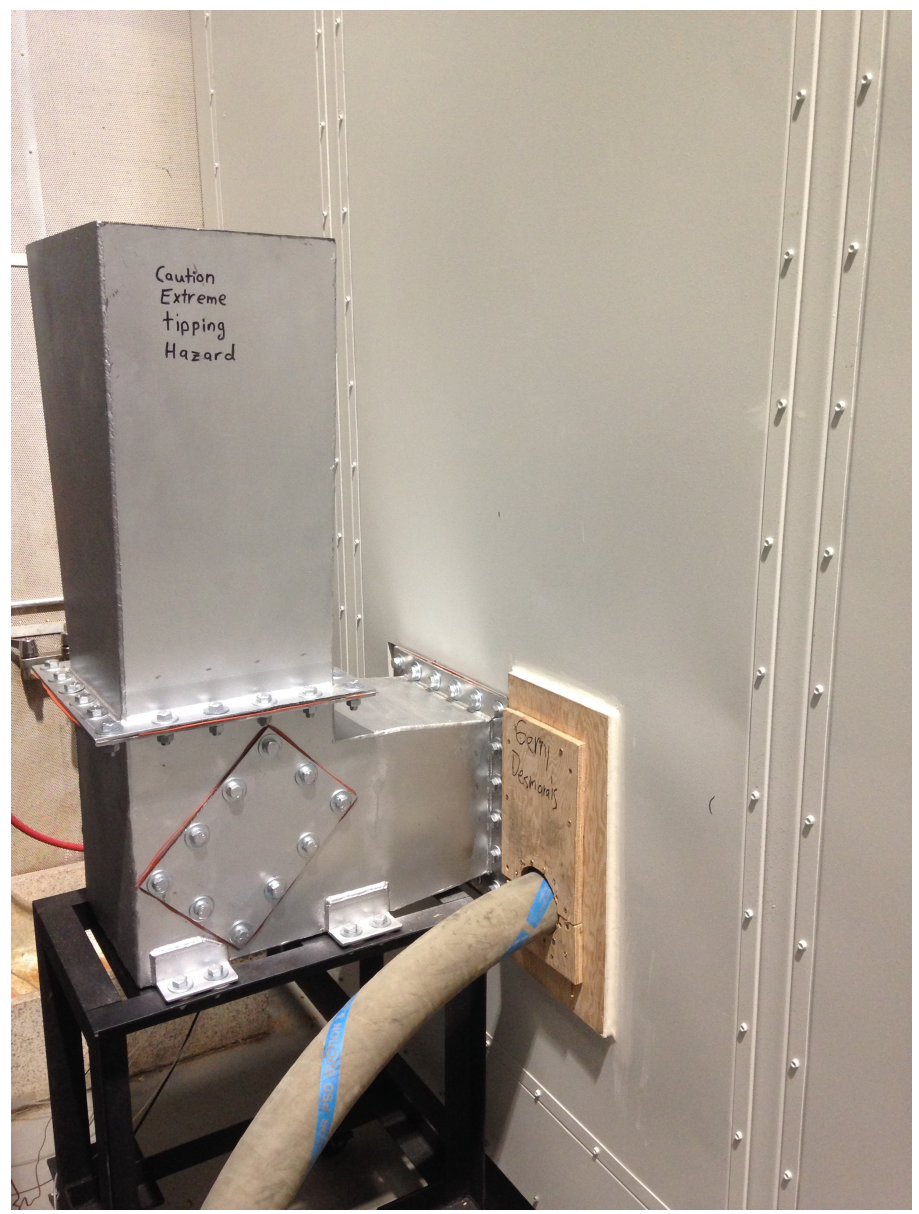

Figure 4.6: The modified access panel for hose access with the external portion of the collector assembly visible

\subsection{Pratt and Whitney High Speed Wind Tunnel}

The air is supplied to the scale model by the Pratt and Whitney High Speed Wind Tunnel. The tunnel has been at Carleton since the early 1990's upon being purchased from McMaster University and commissioned by Jeffries [83]. Many changes have been made since then and the most recent documentation of its state is the 2015 thesis of Kibsey [84]. A schematic of the facility is shown in figure 4.7.

The HSWT is an intermittent blow down wind tunnel used primarily for turbine cascade research. The tunnel is capable of reaching supersonic speeds in the cascade 


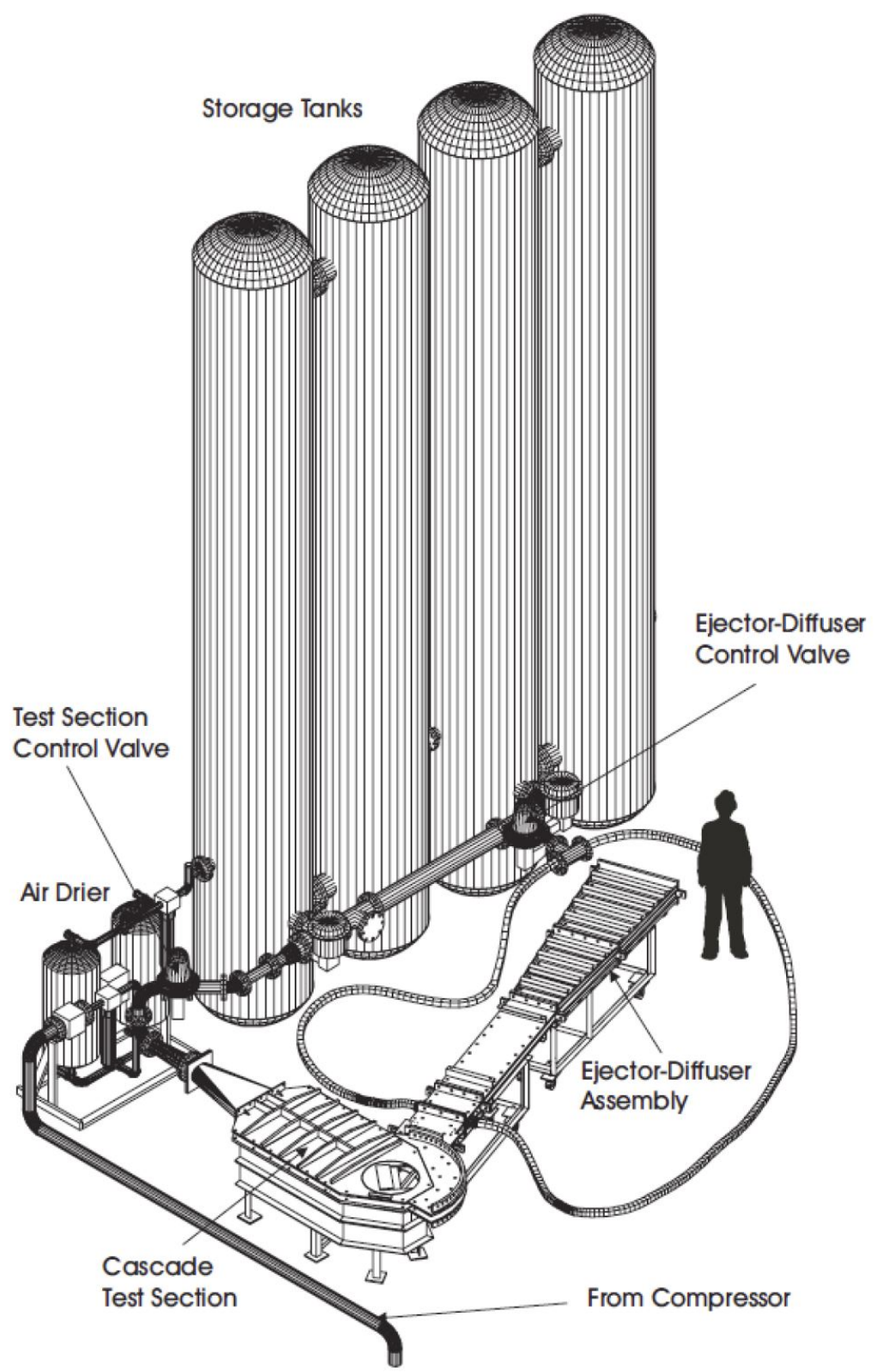

Figure 4.7: A schematic of the Pratt and Whitney High Speed Wind Tunnel, From 84

test section by discharging 4 large compressed air storage tanks, each of 6.5 cubic meters, at a controlled rate. The tanks are filled by a Broom-Wade model VC500 two-stage intercooled positive-displacement compressor located in the basement of the building, shown schematically in figure 4.8, It does not appear this way on the 
figure but the compressor is roughly 6 feet (1.8 meters) high. The compressor uses a 100 horsepower $(73.5 \mathrm{~kW}) 3$ phase motor to deliver air at a maximum pressure of 100 pounds per square inch (psi) $(690 \mathrm{kPa})$ and at a flow rate of roughly 500 standard cubic feet per minute (14.2 cubic meters per minute). From empty, a full charge takes roughly 20 minutes, however, test runs do not completely deplete the tank pressure. In addition, the compressor automatically engages when the tank pressure drops below a threshold of roughly 85 psi $(586 \mathrm{kPa})$ decreasing the net tank mass outflow. The compressor elevates the air temperature thus the air is passed through a reverse flow aftercooler. The aftercooler causes condensation in the air thus it is passed through a moisture separator.

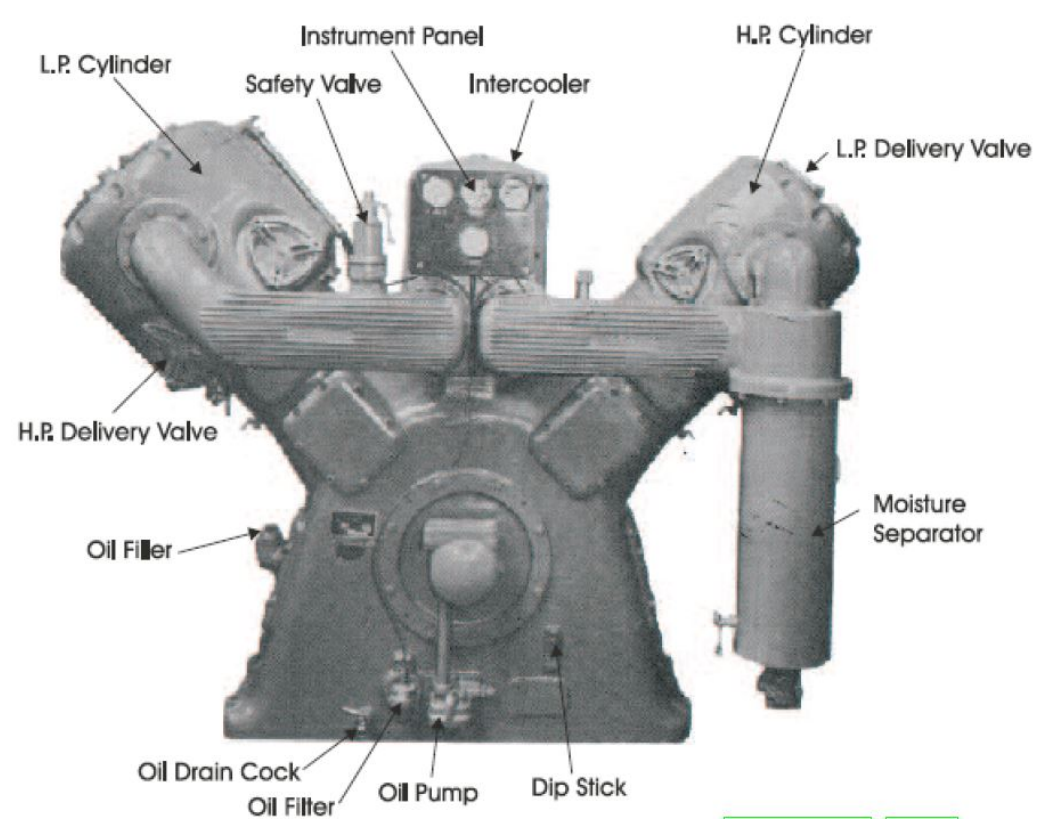

Figure 4.8: A schematic of the Broom-Wade compressor, From [84]

Upon operation the air in the tanks drops in temperature and the air is accelerated which further decreases the temperature. A Comp-Air Kellog model CDH520 regenerative desiccant air dryer is used to lower the dew point to near $-45^{\circ} \mathrm{C}$ to 
prevent condensation up to Mach numbers of 1.2 .

The air discharged from the tanks can take one of two paths, through the main test section or through the auxiliary path (labeled Ejector-Diffuser Control Valve on figure 4.7). The main flow path normally leads to the cascade test section, however, this portion of the tunnel is not used in this work and thus is not discussed further. The auxiliary path is typically used to control the cascade test section Reynolds number, to run a probe calibration rig and more recently to operate a Schlieren equipped nozzle for an undergraduate lab. The auxiliary system is used for all of the scale model tests in this work as it has a smaller control valve allowing for more precise control and it is already equipped with flexible air hoses required to deliver the air to the scale model.

In the auxiliary configuration the air discharging from the tanks first passes through a 6 inch $(153 \mathrm{~mm})$ Neles-Jamesbury 815L butterfly valve with a NelsJamesbury QP3C-M actuator. This combination acts as an on-off safety valve. A 2 inch $(51 \mathrm{~mm})$ Nels-Jamesbury R21 segmented ball valve with a Neles-Jamesbury QP1C-M actuator and a Neles-Jamesbury NE724 0-20 milliamp electro-pneumatic positioner is located a short distance downstream of the shutoff valve and is used to control the air velocity by use of a feedback control system. Upon leaving the control valve the air is normally directed into a T-junction and into two flexible Sanitary Couplers Inc. Protector PSD air hoses and to the intended location. For scale model tests one of the two hoses is removed from the T-junction and the second hose is connected to the scale model through the acoustically treated opening in the anechoic chamber. The end of the hose has a flanged connection which bolts to a matching flange on the primary nozzle. The roughly 25 foot (7.6 meters) long 3 inch $(76.2$ $\mathrm{mm}$ ) inner diameter hose provides a practical method for the air to be fed to the scale model inside the anechoic chamber with minimal interference to the other tests 
performed with the HSWT.

\subsection{Facility Layout}

Figure 4.9 shows the floor plan of the HSWT in the scale model test configuration. All components relevant to this work are visible and labelled.

\subsection{HSWT Velocity Control System}

\subsubsection{Introduction and Basic Operation Principle}

As mentioned previously, the facility is a blow-down wind tunnel thus the supply pressure decreases throughout a given run until a point when the pressure is no longer adequate to provide the desired velocity or the velocity undergoes large fluctuations due to the control scheme. The wind tunnel control is performed via a Labview code which uses the average of 200 points sampled at $2 \mathrm{kHz}$, the same as done by Hall [85] as recommended by Jeffries [83]. This is done in order to minimize the impact of high frequency oscillations or turbulence. The code works by calculating the Mach number based on absolute total and static pressure readings and comparing the resulting Mach number to the target Mach number. The difference between the two is used to calculate an adjustment to the voltage supplied to the valve. This voltage, ranging between 0-10 volts, is supplied by the control computer data acquisition card. The valve positioner requires a current supply, with $0 \mathrm{~mA}$ being fully closed and $20 \mathrm{~mA}$ being fully opened, thus the output voltage is converted to an appropriate current by a Omega OM7-39-02-C voltage to current converter. Figure 4.10 shows the control system operation principle. 


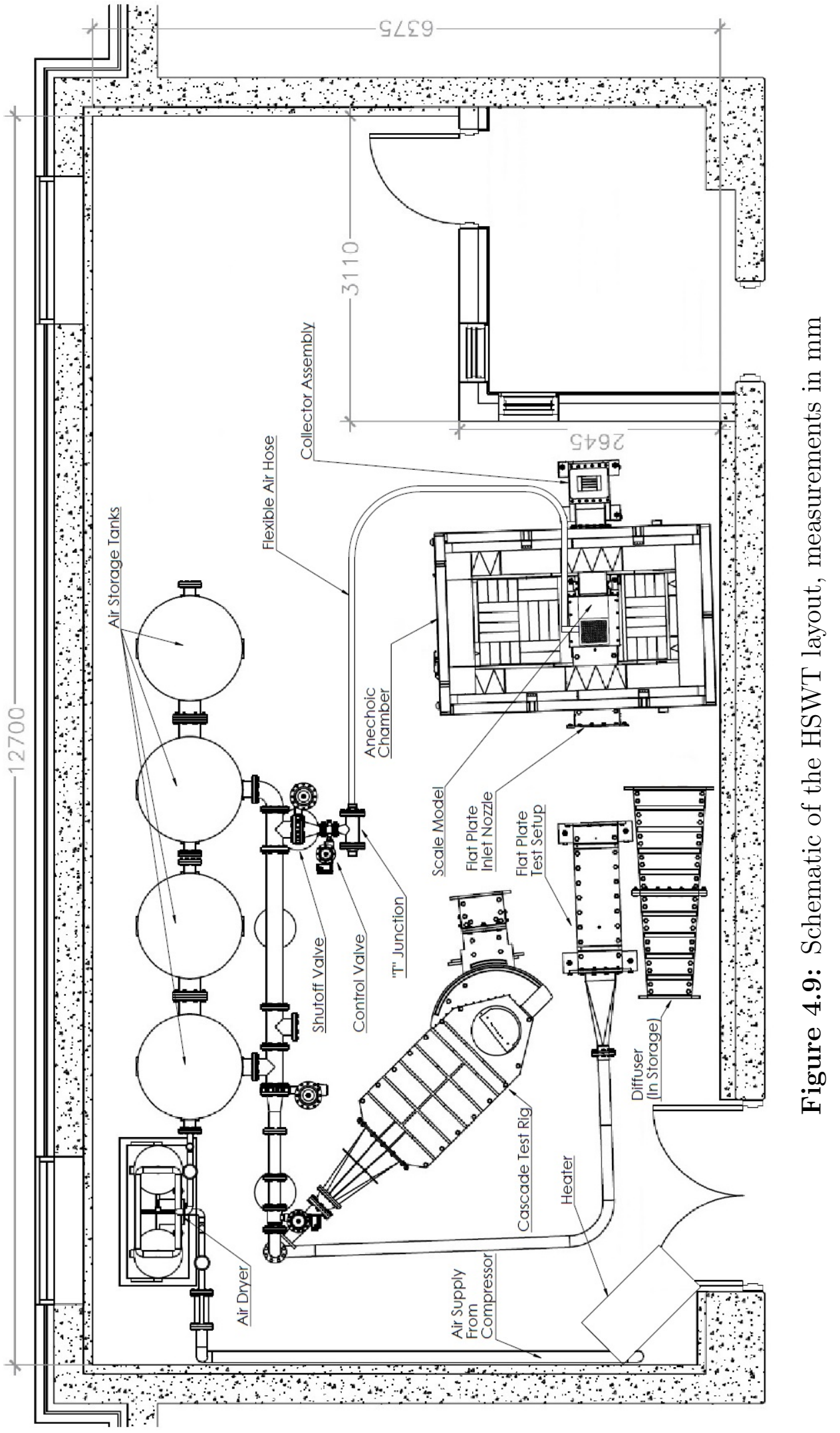




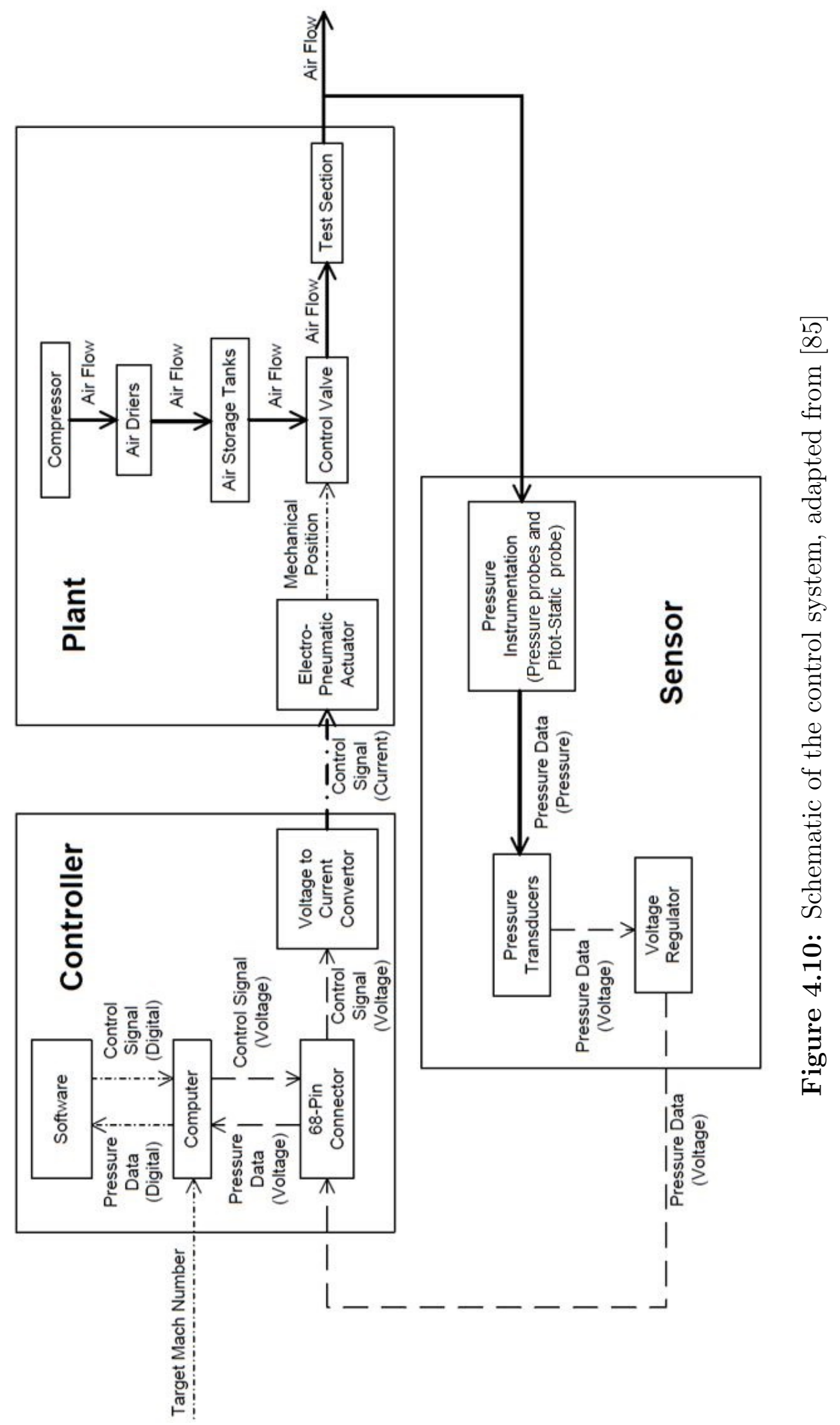




\subsubsection{Pressure Measurements for Control}

A total pressure measurement made between the flanged connection of the flexible hose and the primary nozzle is used in conjunction with a static pressure measurement in the secondary flow chamber in order to control the primary nozzle velocity.

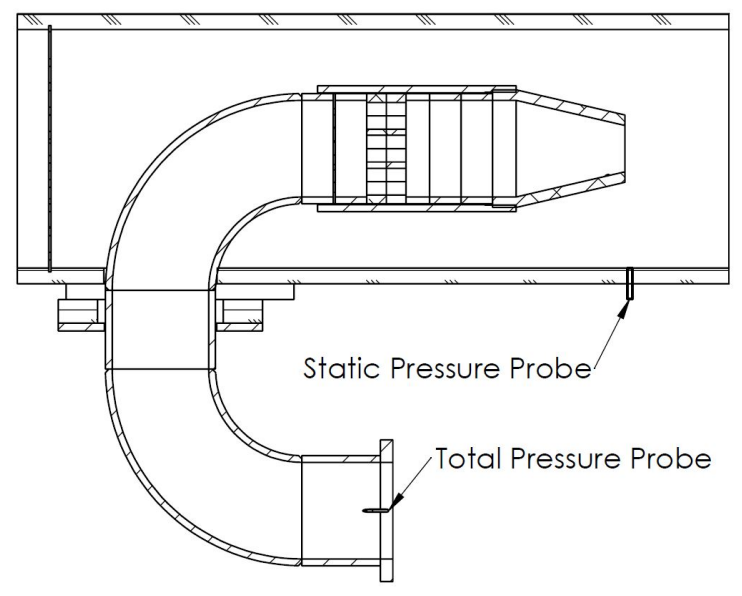

Figure 4.11: A cross sectional schematic showing the location of total and static pressure readings used for velocity control

These two pressure measurements, in combination referred to as the primary nozzle pressure ratio, are used to control the position of the control valve. The total and static pressures are related to the Mach number through the isentropic relation:

$$
\frac{P}{P_{0}}=\left(1+\frac{\gamma-1}{2} M^{2}\right)^{\frac{-\gamma}{\gamma-1}}
$$

where $P$ is the absolute static pressure, $P_{0}$ is the absolute total pressure, $\gamma$ is the ratio of specific heats (1.4 for room temperature air) and $M$ is the Mach Number. For control, the Mach number is the parameter of interest thus equation 4.1 can be rearranged giving the Mach number as a function of the pressure ratio:

$$
M=\sqrt{\frac{2}{(\gamma-1)}\left(\left(\frac{P_{0}}{P}\right)^{\frac{(\gamma-1)}{\gamma}}-1\right)}
$$




\subsubsection{Mach Number Calibration}

Using the measured total and static pressure values in equation 4.2 would give a Mach number greater than the true Mach number given that the total and static pressure measurements are made at different streamwise locations and that they are separated by components causing a pressure drop (flow conditioning, elbows etc.). The relationship can still be used given that the Mach number remains a function of the primary nozzle pressure ratio but a correction must be applied. For this reason, the primary nozzle pressure ratio is calibrated using a pitot-static probe placed in the potential core of the primary nozzle outlet.

The Theory of Turbulent Jets by Abramovich [86] describes the potential core of a jet as a non-viscous region between the boundary layers of the jet. Essentially this is a region of the jet immediately downstream of the primary nozzle contraction, which decreases in area with increasing distance from the nozzle. The velocity in this area is not affected by the surrounding boundary layer and thus it is representative of the maximum jet velocity.

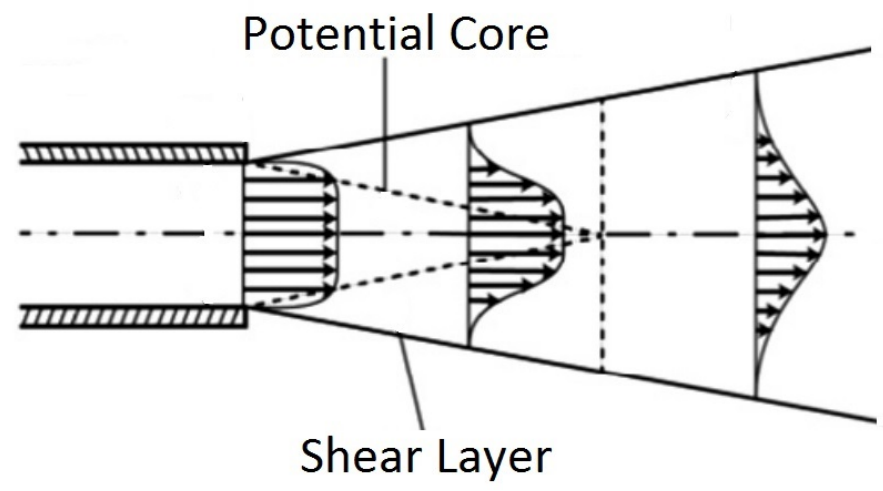

Figure 4.12: Schematic showing the potential core of a free jet

If the pitot-static probe were placed outside this region the measured velocity would not be representative of the primary nozzle velocity. The length of the potential 
core for turbulent compressible axisymmetric subsonic jets varies between roughly 4 and 8 nozzle diameters and is dependent on the particular experiment [87]. A United Sensor PCD-8-KL Pitot-static probe is placed with the tip axisymmetrically centered to the primary nozzle, roughly 0.5 nozzle diameters downstream of the nozzle outlet plane such that both the total and static pressure holes should be well within the potential core. By recording both the primary nozzle pressures and the Pitot-static probe pressures at various nozzle Mach numbers, a calibration between the total pressures can be obtained. The same is true of the static pressures. These calibrations are used to correct the primary nozzle pressure readings to provide a Mach number equal to that measured by the Pitot-static probe. After calibration, the Pitot-static probe is removed and the calibrated primary nozzle pressure ratio is used to control the velocity.

\subsubsection{Potential Calibration Issues}

It is worth noting is that the lengths of tubing used to connect the pressure probes to the pressure transducers have a tendency to damp out velocity fluctuations and impact their phase. This is not characterized in this work but should be kept in mind. The Pitot-probe may also induce turbulence in the potential core thus falsifying the static pressure reading which is taken a short distance downstream of the total pressure. In addition, the Pitot-static probe used is not specifically designed for high-velocity flows and unfortunately the manufacture provides no clear calibration information for these high speed flows. Ideally, the probe would be calibrated in a known flow, however, given the high subsonic flow velocities used this calibration becomes quite expensive. For these reasons the pitot probe measurements are used as-is without any corrections applied. 


\subsubsection{PID Control}

Proportional Integral Derivative (PID) control - with proportional, integral and derivative gains, $K_{P}, K_{I}$ and $K_{D}$ respectively - is used to provide the adjustment to the valve voltage, based on total and static pressure readings. Hall provides recommended $K_{P}, K_{I}$ and $K_{D}$ values of 4,1 and 1 respectively for the auxiliary flow path. It is important to note that the ideal gains vary with Mach number and in addition the uniqueness of the primary nozzle may lead to these gains being inappropriate. The gains for this research are discussed in depth in chapter 6 .

\subsubsection{Modifications by Hall and Giardino for Improved Op- eration}

Hall performed major modifications to the control system in order to obtain longer run times, less velocity fluctuations and better control at low Mach numbers. It was found that a minimum voltage of 3.5 volts was required for there to be any airflow in the auxiliary system thus an appropriate compensation - termed "slack" - was added. A secondary compensation is put in place for the auxiliary valve "dead band", a hysteresis effect when the auxiliary flow path control valve changes direction. The Mach number was found to undershoot the target velocity therefore a Mach number undershoot compensation (which itself is a function of the Mach number) was added to the code to reduce steady state error. While these are the upgrades of interest to this work, many more modifications were undertaken and the details can be found in the thesis of Hall [85]. The modifications result in better controllability and longer run times. Despite this, Hall outlines the importance of monitoring and recording the wind tunnel velocity given that in certain situations the Mach number performance can be peculiar (phenomena such as large fluctuations but only near the end of a 
run).

Giardino used the existing code as a basis for a new code specific to the Aeroacoustics research group. The new version removes advanced functions (relating to cascade testing) not required by the Aeroacoustics group as well as simplifies data logging, allows for pressure ratio calibration and simplifies transitioning from the main flow path to the auxiliary path. The code from Giardino is used for control throughout this work.

\subsection{Instrumentation}

The instrumentation used in this project is subdivided into three systems:

- The pressure measurement system

- The temperature measurement system

- The microphone measurement system

Figure 4.13 provides a block diagram of the overall instrumentation setup and the interrelation of the three systems. In addition to these three systems, an Omega PX613-150G5V 0-150 psig (0-1034.2 kPa (g)) absolute pressure transducer monitors the tank pressure and displays the value on an Omega TX82B current loop indicator digital readout in the control room. This readout is only used to provide the operator with an approximation of the tank pressures and it is not recorded. A USB connected Mobius ActionCam video camera capable of 1080p video is mounted in the chamber to monitor tests. 

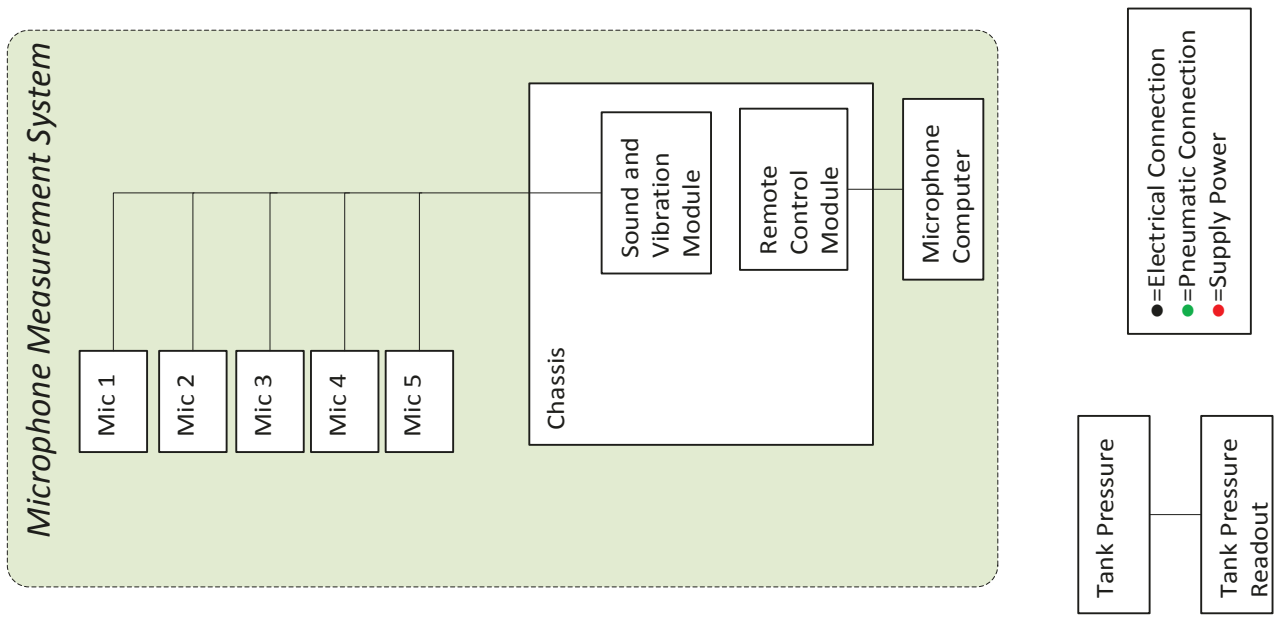

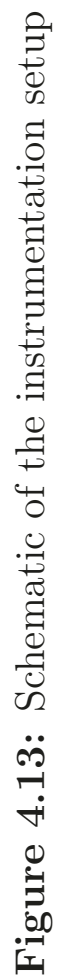

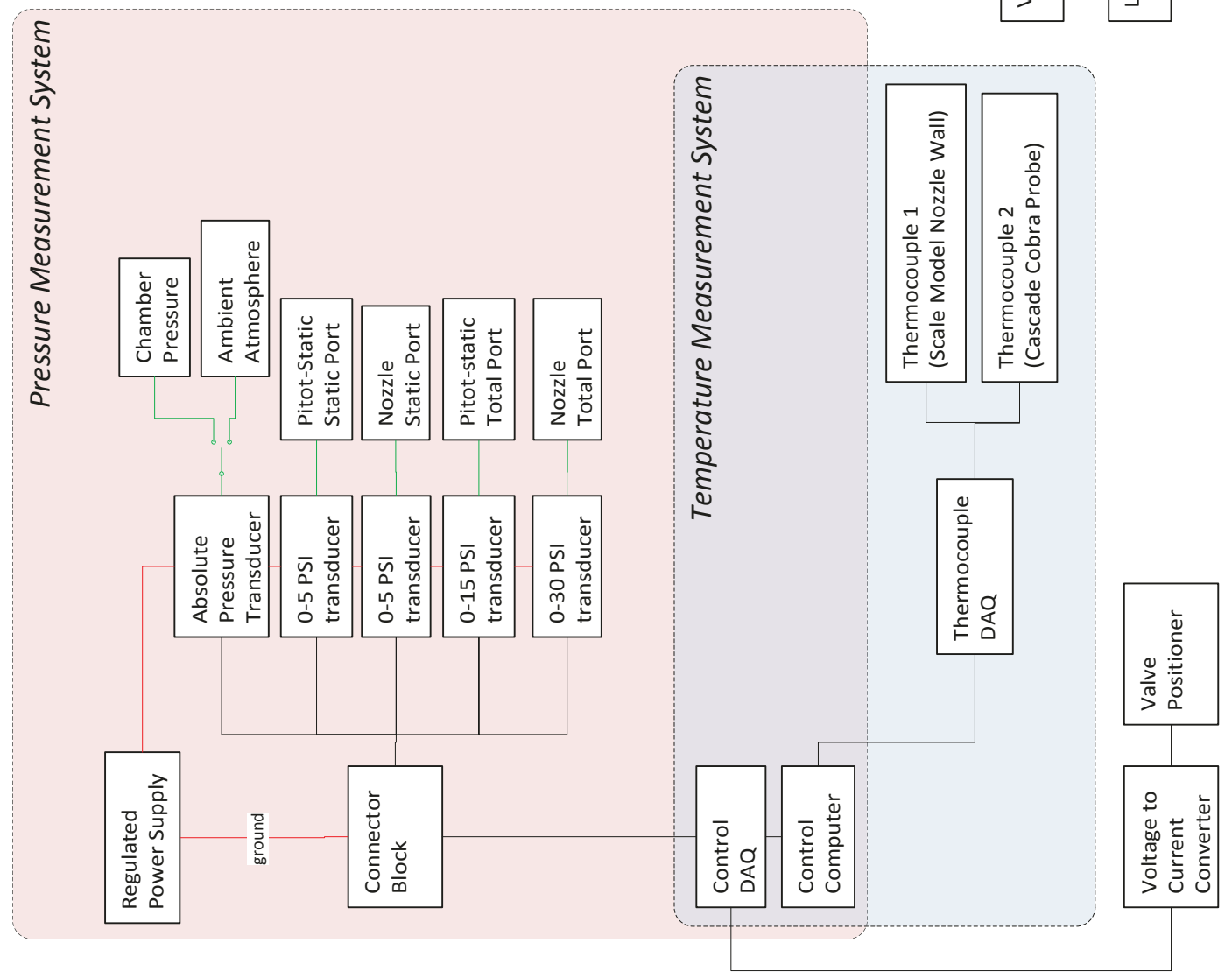




\subsubsection{Pressure Measurement System}

The primary nozzle pressure ratio and Pitot-static pressures are measured using 4 Omega PX140 series pressure transducers. The details of these transducers are provided in table 4.1. The transducers measure a differential pressure and output a voltage dependent on the pressure. The output voltage is nominally between 1 to 6 volts $\mathrm{DC}$, however, this voltage varies with the input voltage which has an acceptable range of 7 to 16 volts DC. For this reason the input voltages are set and maintained at the recommended 8 volts DC by a single Omega PST-4130 regulated power supply.

Table 4.1: Differential Pressure Transducers' Information

\begin{tabular}{|c|c|c|c|}
\hline Number & Measurement & Transducer & Type \\
\hline 1 & Primary Nozzle Total Pressure & PX142-030D5V & Differential \\
\hline 2 & Primary Nozzle Static Pressure & PX142-005D5V & Differential \\
\hline 3 & Pitot-Static Total Pressure & PX142-015D5V & Differential \\
\hline 4 & Pitot-Static Static Pressure & PX142-005D5V & Differential \\
\hline Number & r Calibration Coefficients a \& b & \multicolumn{2}{|c|}{ Range } \\
\hline 1 & 6.0284 \& Measured Daily & \multicolumn{2}{|c|}{ 0-30 psi (0-206,843 Pa) } \\
\hline 2 & $0.9961 \&$ Measured Daily & \multicolumn{2}{|c|}{$0-5$ psi $(0-34,474 \mathrm{~Pa})$} \\
\hline 3 & 3.0126 \& Measured Daily & \multicolumn{2}{|c|}{ 0-15 psi $(0-103,421 \mathrm{~Pa})$} \\
\hline 4 & $0.9958 \&$ Measured Daily & \multicolumn{2}{|c|}{ 0-5 psi $(0-34,474 \mathrm{~Pa})$} \\
\hline
\end{tabular}

A linear calibration is used to calculate the pressure from the voltage:

$$
P=a V+b
$$

where $\mathrm{P}$ is the measured pressure, $\mathrm{V}$ is the transducer output voltage and a and $\mathrm{b}$ are the calibration coefficients of slope and offset respectively. Giardino found that 
the offset, b, of the differential transducers was found to vary day to day (believed to be due to atmospheric variations) thus implemented a calibration scheme following equation 4.4 (differential transducers only):

$$
P=a(V-b)
$$

The calibration coefficients are found through an in house static sequential calibration using a Druck DPI 605 precision portable pressure calibrator, however, the offset $\mathrm{b}$ is adjusted daily. The calibrator is capable of generating a pressure to 300 psig (20 bar) with an accuracy of $\pm 0.025 \%$ of the reading. The pressure measurements are all made with one port of the differential transducer connected to the point of interest and the other port open to atmosphere in the main wind tunnel but outside the chamber. The calibration constants of each transducer are integrated into the LabVIEW data acquisition and control program. The calibration curves for the pressure transducers can be found in Appendix A.

In addition to the aforementioned pressure transducers, a 0-100 psi (0-689,476 Pa) Druck PDCR 22 absolute pressure transducer is used to measure the atmospheric pressure before any given run and also to monitor the chamber static pressure during runs. This transducer follows the calibration in equation 4.3 and has calibration coefficients of 1338.83 and -1.21214 for a and b respectively. The calibration curve can be found in Appendix A.

The pressure transducers are wired into a National Instruments SCB-68 shielded I/O connector block. This connector block is used in the default switch configuration and is connected to a 16 bit National Instruments PCI-6229A data acquisition card, mounted in a desktop computer (the control computer) running Windows XP Professional with $2 \mathrm{~GB}$ of ram and an Intel Pentium $43.0 \mathrm{GHz}$ processor and LabVIEW 8. 
This data acquisition card also has analogue output capabilities and thus it is used as the voltage source for controlling the valve position.

\subsubsection{Temperature Measurement System}

The air temperature is not normally monitored for any given run, however, the temperature is measured for baseline characterization. The flow temperature is estimated with a type $\mathrm{K}$ thermocouple which is secured to the outside of the primary nozzle. Although not indicative of the true flow temperature, it provides an approximation. The ambient temperature is also recorded using a type $\mathrm{K}$ thermocouple within the cobra probe used by Jeffries and Hall, located in the cascade test section. Both thermocouples are connected to a National Instruments USB-9211A data reader which is connected to the control computer via USB. The thermocouples follow the default manufacture calibrations.

\subsubsection{Microphone Measurement System}

5 high performance Brüel \& Kjær (B\&K) 4944-A, 1/4 inch (6.35 mm) pressure field microphones with built in DeltaTron preamplifiers are used in order to capture the acoustic phenomena in the scale model. The specific microphone details are provided in table 4.2. These microphones have a flat frequency response from roughly 50-10,000 $\mathrm{Hz}$ with a typical response curve (no cap configuration) shown in figure 4.14.

The microphones have a maximum sound pressure limit of 182 decibels with a nominal sensitivity of $0.9 \mathrm{mV} / \mathrm{Pa}$. Each microphone is labeled 1 through 5 and is always connected to the data acquisition system with its respective AO 0587-D-100 connection wire. The microphones measure only fluctuating pressures, thus explaining 
Table 4.2: Microphone Details

\begin{tabular}{crc} 
Microphone Number & Serial Number & Sensitivity $(\mathrm{mV} / \mathrm{Pa})$ \\
\hline 1 & 2931836 & 0.830 \\
2 & 2931839 & 0.819 \\
3 & 2931840 & 0.897 \\
4 & 2931837 & 0.851 \\
5 & 2931838 & 0.848
\end{tabular}

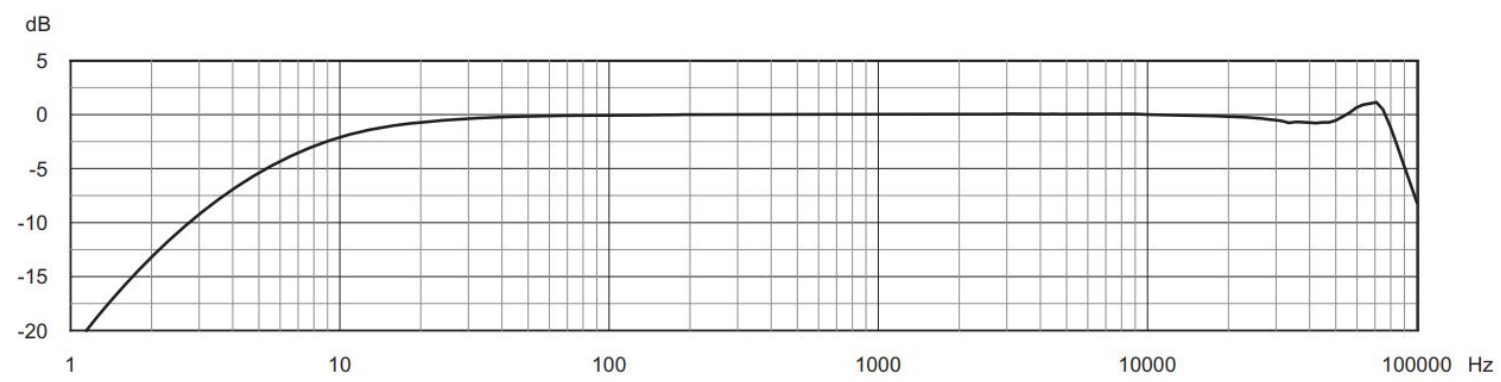

Figure 4.14: Typical 4944-A microphone frequency response, from [88]

the low-frequency roll off. The fluctuations are measured between the front of the microphone and an internal cavity. The microphones follow a linear calibration similar to equation 4.3. For the microphones $\mathrm{a}$ is the sensitivity and $\mathrm{b}$ is set to 0 . In postprocessing it is found that the average pressure is normally on the order of $10^{-6}$, which is expected given that the microphones record fluctuating pressures. This average is much smaller than the recorded pressure values (up to 1,200 Pa) thus it is deemed negligible, explaining why b is 0 .

No calibration tool is available for these microphones therefore the manufacture provided sensitivities are used. Van Blitterswyk, a former research assistant, explained that a B\&K representative visited Carleton University in the Fall of 2014 with a portable microphone calibration unit (such as the B\&K 4231) and found that 
the manufacture provided sensitivities were still be valid thus they were used throughout his work ${ }^{3}$. In addition an attempt at an in-house calibration showed results very close to aniticipated ${ }^{4}$ further validating the use of the manufacture provided sensitivities. The attempted in-house calibration plots for the microphones can be found in Appendix A.

The microphone data is acquired using a 24 bit, 8 analogue channel National Instruments PXIe-4492 Sound and Vibration module housed in a PXIe-1071 chassis with a PXIe-8381 Remote Control Module. The data is recorded on a computer running Windows 7 Enterprise with 16 GB of ram and an Intel Core i7-4770 $3.4 \mathrm{GHz}$ processor using LabVIEW 13.

\subsection{Measurement Uncertainty}

Giardino and Hall both discuss the uncertainty of measurements made in their work with Hall using the method presented by Kline and McClintock [89] and Giardino using that of Tavoularis [90]. Although this work uses many of the same transducers making the same measurements, the results are not directly applicable given the different magnitudes measured.

The uncertainty, $\delta$, in a calculated value, $F$, can be found from the independent variables, $\phi_{1}, \phi_{2}, \phi_{3}, \ldots$, from which $\mathrm{F}$ is calculated along with their respective uncertainties, $\delta \phi_{1}, \delta \phi_{2}, \delta \phi_{3}, \ldots$, using equation 4.5 .

$$
\delta F=\sqrt{\left(\frac{\partial F}{\partial \phi_{1}}\right)^{2} \delta \phi_{1}^{2}+\left(\frac{\partial F}{\partial \phi_{2}}\right)^{2} \delta \phi_{2}^{2}+\ldots+\left(\frac{\partial F}{\partial \phi_{n}}\right)^{2} \delta \phi_{n}^{2}}
$$

The simplest transducer uncertainty calculation in this work is the atmospheric

\footnotetext{
${ }^{3}$ Private communication between Van Blitterswyk and the author
} ${ }^{4}$ see chapter 7 
pressure uncertainty. Recall equation 4.3 for calculating the pressure from a voltage, repeated here for clarity.

$$
P=a V+b
$$

The uncertainty in the measured quantity, $P$, is a function of the independent variables, $a, V$ and $b$, from which it is calculated and their uncertainties. The partial derivatives of $P$ with respect to $a, V$ and $b$ are required. These are:

$$
\frac{\partial P}{\partial a}=V \quad \frac{\partial P}{\partial V}=a \quad \frac{\partial P}{\partial b}=1
$$

The values of $\mathrm{a}$ and $\mathrm{b}$ come from the linear best fit line of the transducer calibration curve. For a given pressure, the expected voltage can be estimated (or vise versa, but it is more convenient to set the expected pressure). The uncertainty of the curve fitting coefficients (the calibration coefficients), $\delta a$ and $\delta b$, are found using Excel's linest $t^{5}$ function. The uncertainty is taken as twice the standard deviation of these coefficients, the same as done by Hall and Giardino, giving a 95\% confidence level. The uncertainty in the measured voltage was estimated by Jeffries as $\pm 0.25 \%$ of the reading. For a standard atmospheric pressure, $14.696 \mathrm{psi}(101,325 \mathrm{~Pa})$, the uncertainty in the atmospheric pressure measurement, $\delta P_{a t m}$, can be calculated using the appropriate values from table 4.3 and equation 4.5 .

$$
\begin{array}{r}
\delta P=\sqrt{\left(\frac{\partial P}{\partial a}\right)^{2} \delta a^{2}+\left(\frac{\partial P}{\partial V}\right)^{2} \delta V^{2}+\left(\frac{\partial P}{\partial b}\right)^{2} \delta b^{2}}=\sqrt{V^{2} \delta a^{2}+a^{2} \delta V^{2}+1^{2} \delta b^{2}} \\
= \pm 0.196 \operatorname{psi}(a)
\end{array}
$$

\footnotetext{
${ }^{5}$ Calculates the statistics of a best fit line obtained using the least squares method
} 
Table 4.3: Values Required for the Uncertainty Analysis for the Atmospheric Pressure Transducer

\begin{tabular}{cc} 
Variable & Value \\
\hline $\mathrm{a}$ & 1338.8 \\
$\mathrm{~b}$ & -1.212 \\
$\mathrm{~V}$ & 0.0119 \\
$\mathrm{P}$ & $14.70 \mathrm{psi}(\mathrm{a})$ \\
$\delta a$ & 14.368 \\
$\delta b$ & 0.0865 \\
$\delta V$ & $2.971 * 10^{-5}$ Volts \\
$\delta P$ & $?$ psi $(\mathrm{a})($ desired value $)$
\end{tabular}

A more convenient form in which to express this result is as a percentage of the reading, that is $14.70 \mathrm{psi}(\mathrm{a}) \pm 1.3 \%$. The amount of uncertainty varies based on the magnitude of the measured quantity, thus this uncertainty calculation is repeated for a range of input pressures leading to a trend for the uncertainty in the measured atmospheric pressure, shown in figure 4.15 .

Although the calculation of uncertainty for a single measured value is straightforward, obtaining the final uncertainty on the primary nozzle Mach number is an involved process due to the Mach number calibration discussed earlier. The primary nozzle Mach number is calculated from the total and static gage pressures of the primary nozzle which are added to the atmospheric pressure, all of which have an uncertainty. In addition, the total and static gage pressures of the primary nozzle are each corrected with an equation derived from a curve fit which has a certain amount of uncertainty. These curves come from measurements of the total and static pressures of the primary nozzle as well as the Pitot-static probe, all of which have uncertainty. Figure 4.16 is a block diagram showing how the pressure measurements are used for 


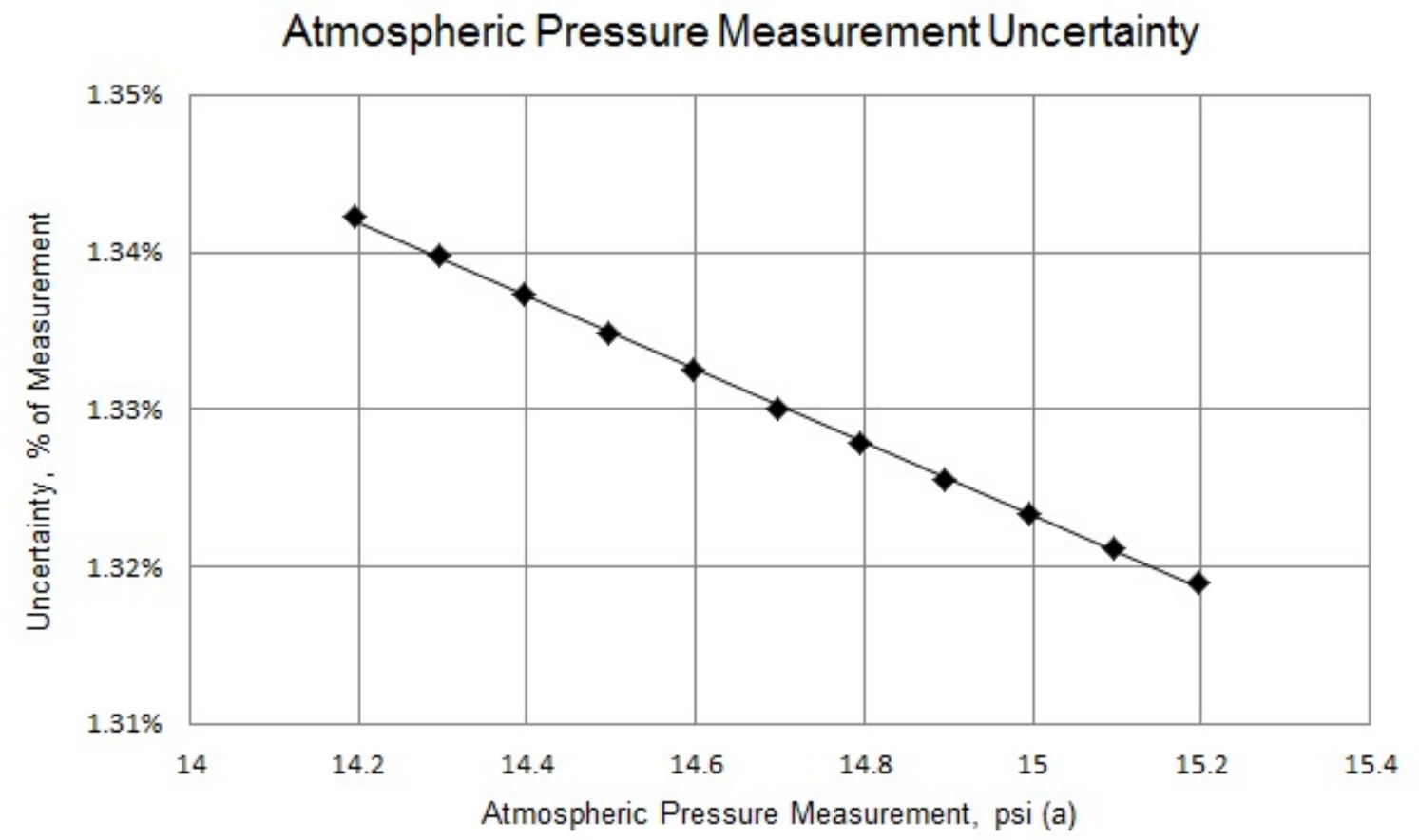

Figure 4.15: The measurement uncertainty estimate for a range of atmospheric pressures

the primary nozzle Mach number calculation. Figure 4.17 presents the estimated uncertainty on the final control Mach number. The intermediate uncertainties are omitted for brevity.

As can be seen, the Mach number uncertainty is found to decrease with increasing Mach number. This is expected as many of the transducer errors are a percentage of the full-scale measurement range. As the Mach number, thus total pressure, becomes larger and larger, the relative measurement error becomes smaller and smaller. Certain assumptions are made in estimating the control Mach number uncertainty including a constant atmospheric pressure, normal distribution of errors, no error in the calibration pressures and no error due to the physical measurement probes. An interesting note is that the uncertainty due to the Mach number fluctuations (discussed 


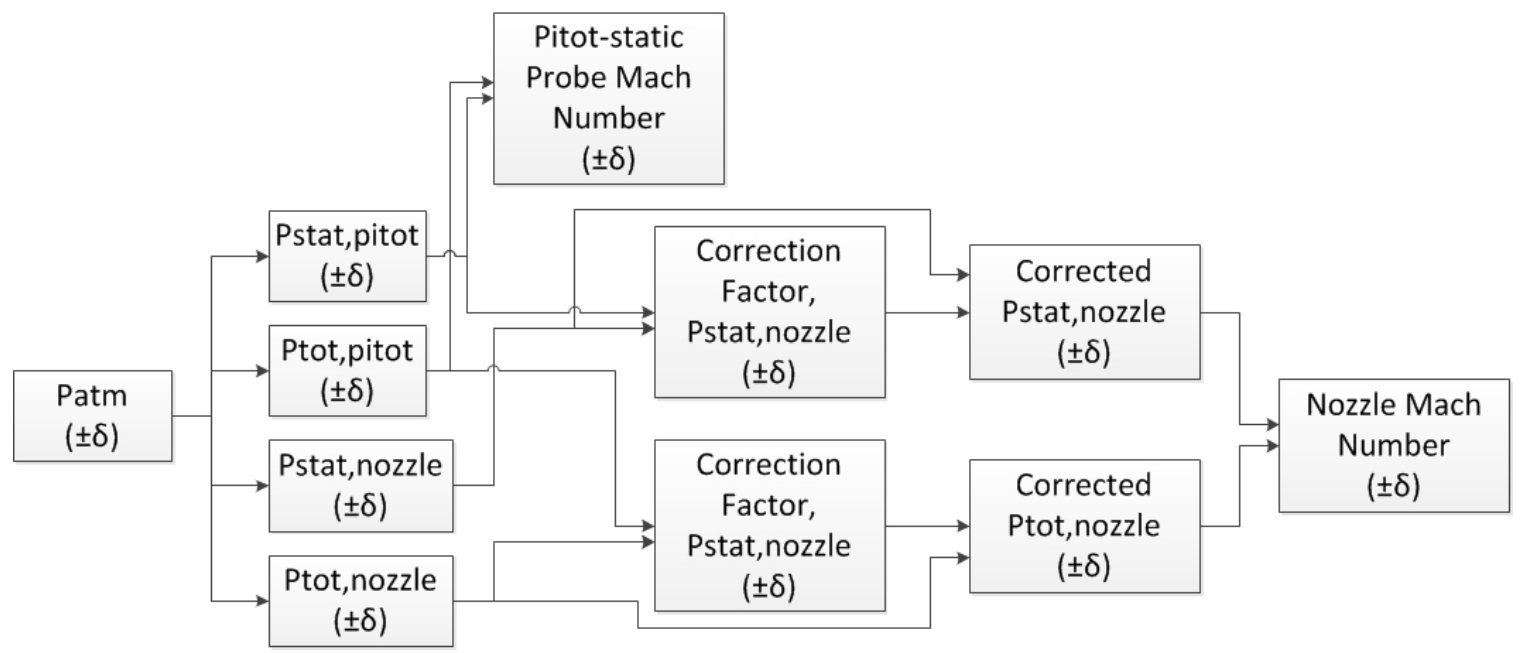

Figure 4.16: Block diagram illustrating the steps required for Mach number calculation

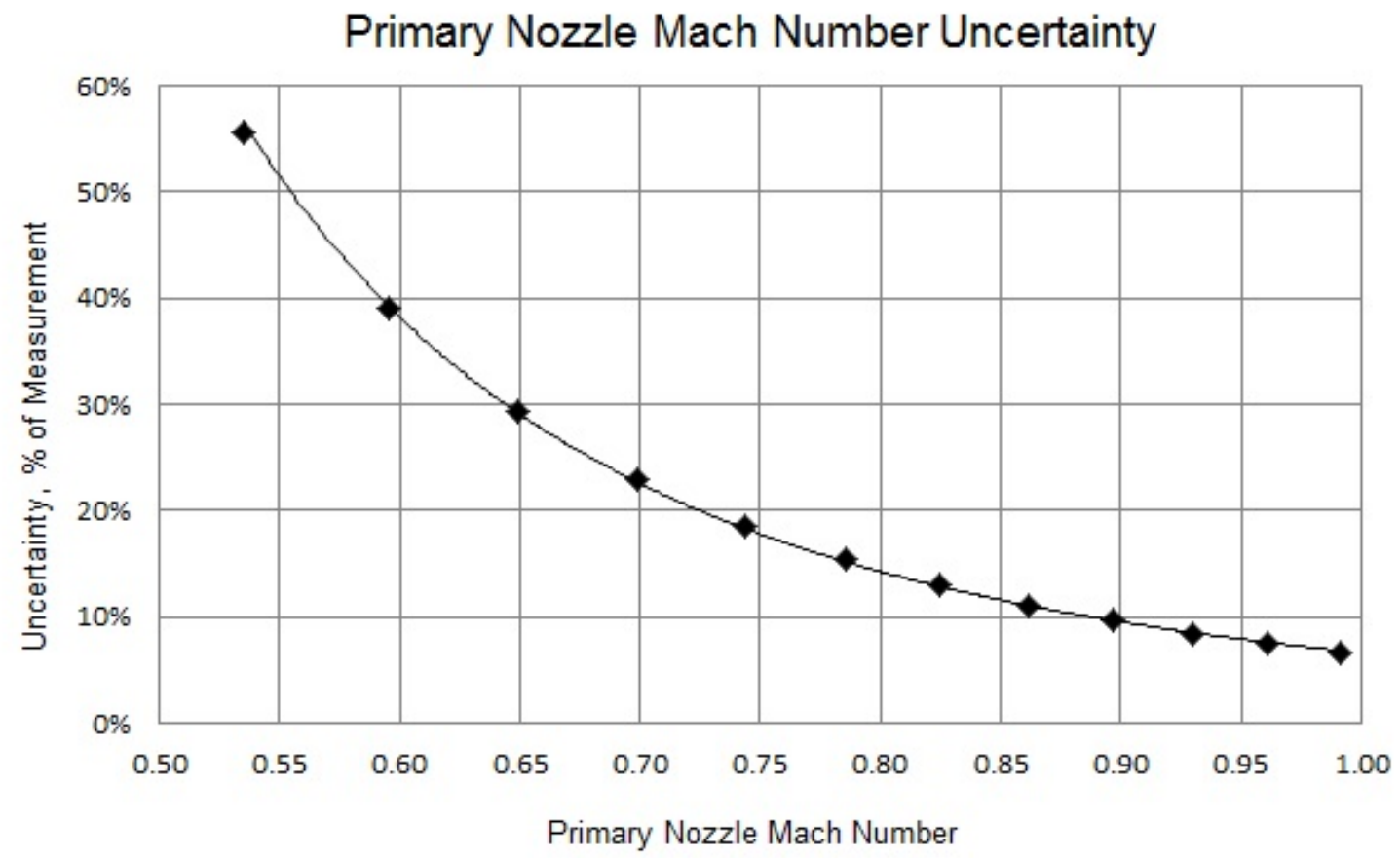

Figure 4.17: The measurement uncertainty estimate for a range of nozzle Mach numbers (with the nozzle measurements used for control)

later) is found to be at least an order of magnitude smaller than the uncertainty stemming from the transducers.

There is also an uncertainty associated with the microphones. This was discussed 
by Van Blitterswyk [91]. The manufacturer's calibration uncertainty is $\pm 0.3 \mathrm{~dB}(1.04$ $\mathrm{Pa}$ ), rel $1 \mathrm{~Pa}$ and this includes the uncertainties relating to the pre-amplifier gain, non-linearity, repeatability and rounding errors. The microphone data acquisition system has an input line voltage uncertainty of $\pm 0.1 \%$ of the full-scale input voltage. The total uncertainty for a microphone with a calibration constant of $0.83 \mathrm{mV} / \mathrm{Pa}$ was estimated to be $4 \%$.

The temperature measurement uncertainty is not considered. 


\section{Chapter 5}

\section{Scale Model Design}

The scale model is fairly complex with more than 50 individual components required

to be manufactured. A schematic of the scale model is shown in figure 4.1. The scale model is entirely modelled using Computer Aided Design (CAD) tools to allow the model to be completely visualized in three dimensions, to inspect part placement and to anticipate any issues which may arise during manufacturing, assembly or operation. Manufacturing and assembly are kept in mind throughout the design process in order to streamline these processes (design for manufacturability and design for assembly). This chapter outlines the mechanical design, acoustic considerations and aerodynamic design of the scale model.

\subsection{Scale Factor Rationale}

While the scale factor (S.F) is an important consideration, the choice of scale factor for this work is somewhat arbitrary. The scale factor can be defined as

$$
\text { S.F. }=\frac{\text { Full scale dimensions }}{\text { Model scale dimensions }}
$$

The main constraints dictating the size of the scale model for this study are: 
- The mass flow rate attainable with the HSWT

- The size of the anechoic chamber collector

- The physical space inside the anechoic chamber

The wind tunnel is capable of providing a mass flow rate far exceeding that required by the largest model useable in the anechoic chamber thus this constraint can be ignored. If the scale model outlet flow chamber were made to the same size as the collector inlet, the scale factor would be approximately 140 which would require such a small mass flow rate that a new control valve would likely be required (mass flow scales to S.F. ${ }^{2}$ ). It is desired to keep the scale model as large as possible to simplify measurements and manufacturing. This results in a scale factor constrained only by the useable space in the anechoic chamber. It is important to leave enough room at the scale model inlet for entrained air to enter freely. The maximum size model which can fit in the chamber with adequate space at the inlet has a scale factor of approximately 70. A scale factor of 70.6 is used as it allows the ejector throat to be made from standard sized pipe. The wind tunnel is capable of providing a mass flow rate approximately 6 times greater than that required at this scale. The relatively low mass flow rate has the advantage that the blowdown tanks empty at a slower rate allowing for longer runtimes.

The scale model is designed to be geometrically similar to a true ejector. This geometric matching leads to an impossibility to match all similarity parameters between a full scale ejector and the scale model. The Mach number is chosen as the similarity parameter to be matched. More specifically, the primary nozzle outlet Mach number is matched to that of a full scale ejector. The acoustic power from a subsonic isothermal jet, $W_{j}$, can be theoretically shown to be 


$$
W_{j}=G \rho_{\infty} u_{j}^{8} c_{\infty}^{-5} d_{j}^{2}
$$

where $G$ is a proportionality constant, $\rho_{\infty}$ is the ambient fluid density, $u_{j}$ is the jet velocity, $c_{\infty}$ is the ambient fluid speed of sound, and $d_{j}$ is the jet diameter [92]. As can be seen, the acoustic power scales to the $8^{\text {th }}$ power of velocity, thus Mach number is an important parameter in jet noise generation. This $8^{\text {th }}$ power relationship was obtained by Lighthill[93] from dimensional analysis and the use of Green's Functions to solve an inhomogeneous wave equation of acoustic wave propagation and sources generating the noise field. The formulation has been found to closely agree with experimentation and is referred to as Lighthill's analogy. This analogy forms the foundation of the field of aeroacoustics. Further details are beyond the scope of this work but the paper by Lighthill (reference [93]) is a recommended starting point for the interested reader.

Changes in temperature throughout a run are more easily accounted for using the Mach number than say velocity or Reynolds number. Using the Mach number as the similarity parameter also prevents supersonic primary nozzle velocities which are inappropriate and may lead to great acoustic contamination through mechanisms such as screech tones.

The inability to match all similarity criteria may be acceptable up or down to a certain scale factor where a similarity parameter varies too much from the full scale equivalent and the flow physics change. For example, as the scale factor is continuously increasing the scale model Reynolds numbers are continuously decreasing. At a given point, the flow will transition from turbulent to laminar and the scale model will no longer be representative of a full scale ejector. Unfortunately there is no information on similarity criteria transition for ejectors or the impact this may have 
on acoustics. Simply for the sake of observation, the scale model primary nozzle Reynolds number (based on nozzle outlet diameter and nominal velocity) is found to be approximately 910,000, well above conventional laminar to turbulent transition values.

\subsection{Material Selection}

Ejectors tend to be made from steel or aluminum. The use of steel in the small scale is possible but inconvenient. Steel is heavy (the anechoic chamber has a weight limit), expensive and relatively difficult to work with. For these reasons, all the panel parts are made from 1/2 inch $(12.7 \mathrm{~mm})$ good one side (G1S) plywood. This material is lightweight, fairly strong, inexpensive and easy to work with. The inlet convergence, throat, diffuser, and cone require a smooth surface finish and must be able to withstand the anticipated flows. They are made of 6061 aluminum for its light weight and ease of manufacturability. The perforated cylinder is made of perforated steel sheet. Steel is chosen for its increased strength and higher Youngs modulus in order to avoid any potential "drumming". In addition, perforated steel sheet is less expensive than aluminum and easier to weld. The inlet and exhaust perforated sheets are also made of steel for these reasons.

\subsection{Modular Design}

The scale model is designed to be highly modular in order to allow for modifications to be easily made. Nearly every component can be swapped out in order to evaluate different configurations. This section outlines the modularity of each individual component. 


\subsubsection{Primary Nozzle}

The primary nozzle is designed such that a variety of flow conditioners and spacers can be inserted and removed with their order easily changed. These components are "sandwiched" between a rear shoulder and the contraction. The contraction is externally threaded and screws into the primary nozzle. The contraction screws in until the flow conditioning devices and spacers are slightly compressed, securing them in place. The contraction can easily be replaced by another to obtain different flows.

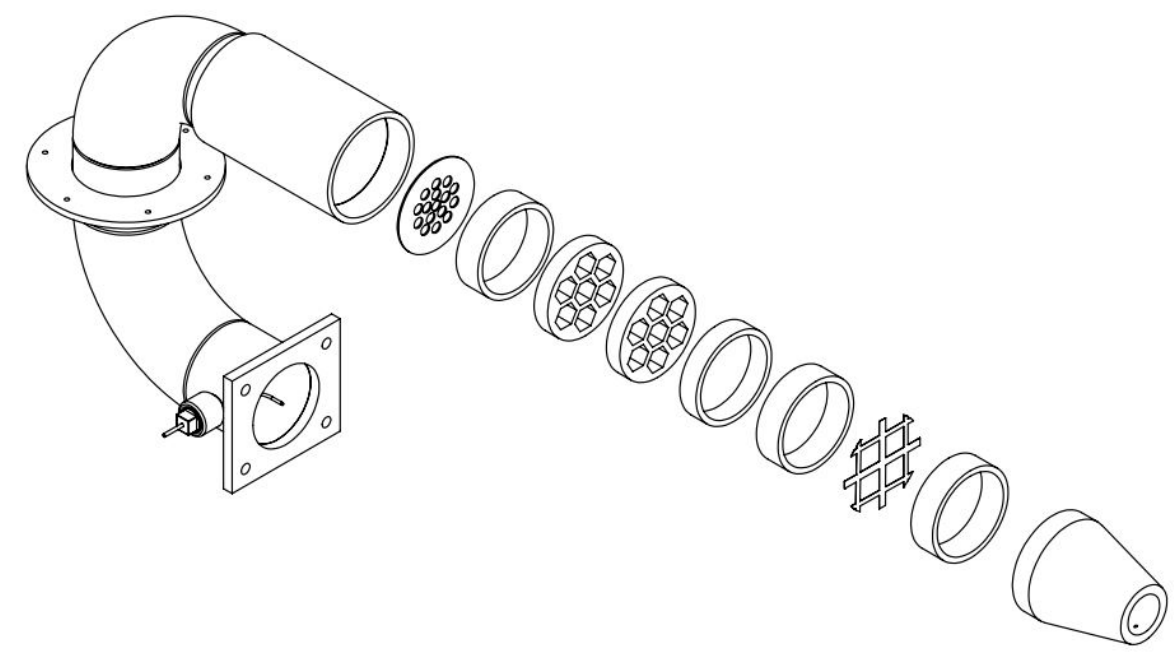

Figure 5.1: A schematic of the primary nozzle showing possible flow conditioners, spacers and contraction

\subsubsection{Throat}

The throat is essentially a section of aluminum pipe with flanges welded on each end. This allows for a new pipe to be used if desired. 


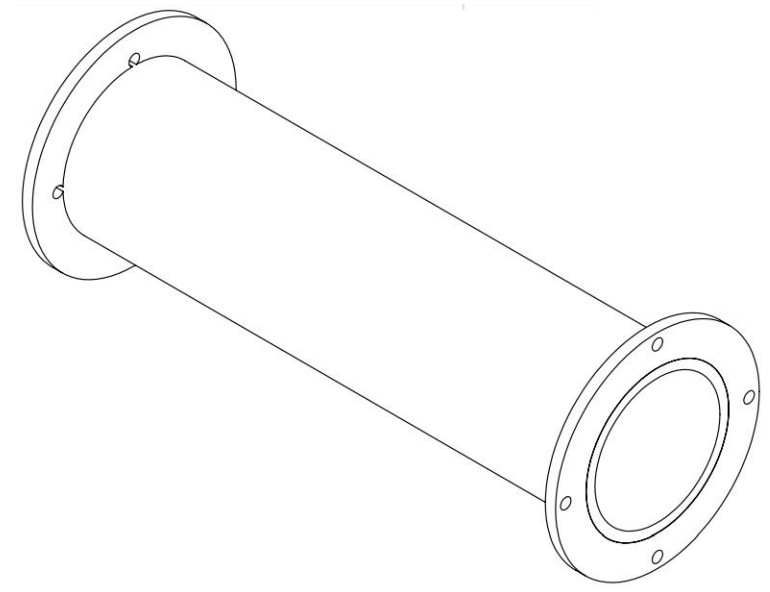

Figure 5.2: Schematic of the scale model throat with flanged ends

\subsubsection{Diffuser}

The diffuser is made of two parts. The outer part connects to the throat flange, is secured to the outlet chamber wall and has an external step to interface with the perforated cylinder. The second part of the diffuser is an internally tapered sleeve which snugly slides in and out of the outer part. The tapered sleeve is held in place with set screws. The sleeve can be swapped in minutes allowing for different diffuser angles and elaborate diffusers to be tested.
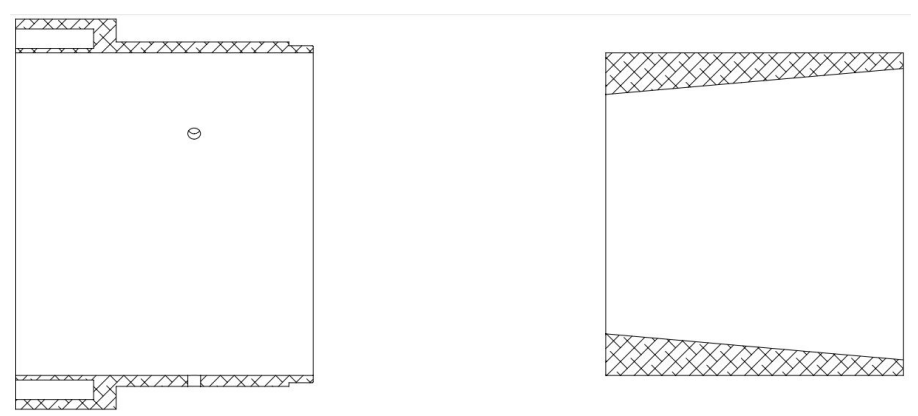

Figure 5.3: Schematic cross section of the two scale model diffuser components 


\subsubsection{Perforated Cylinder}

The perforated cylinder is not secured to the other components by any fasteners or locking devices. The external step in the diffuser is inversely matched in the perforated cylinder allowing for a secure fit. The cone fits inside the opposite end of the perforated cylinder with a slight press fit. The cone is supported by the base assembly and so it has a tendency to push the perforated cylinder against the diffuser securing everything in place. This design allows for the perforated cylinder to be quickly and easily removed by removing the scale model from the base assembly.

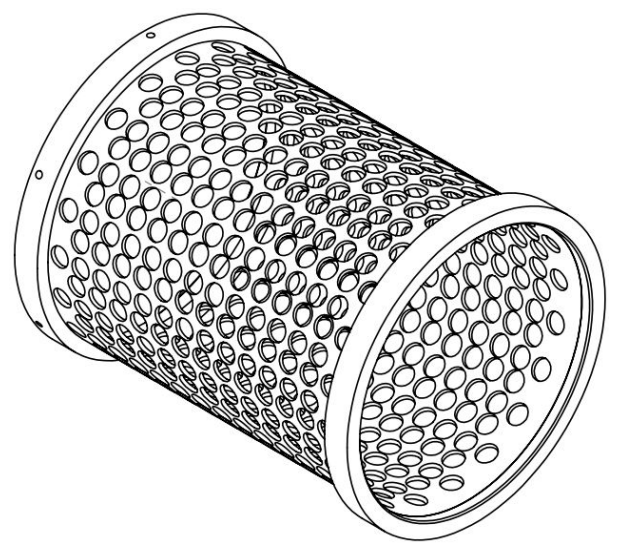

Figure 5.4: A schematic of the perforated cylinder, the left hand side interfaces with the diffuser and the right hand side accepts the cone

\subsubsection{Cone}

Much like the perforated cylinder, the cone is not connected to any other component with fasteners. The cone fits into the perforated cylinder and the cone-cylinder combination fits just right between the diffuser and base assembly. The cone can easily be changed to test a variety of configurations. In addition, a shell in the shape of the cone can be slid on top of the existing cone in order to test different configurations 

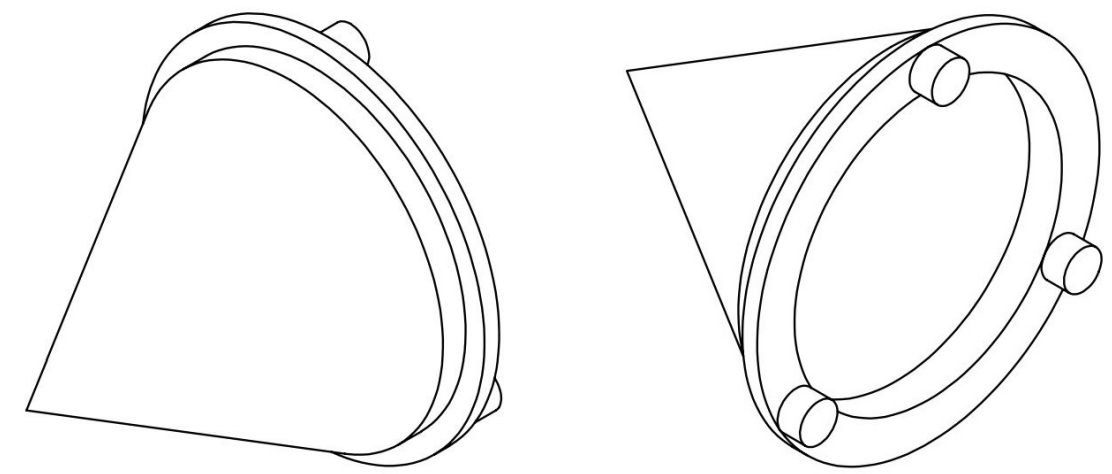

Figure 5.5: An isometric schematic showing the front (left) and rear (right) of the cone

(such as a sandpaper cone to test the effect of a varying surface roughness).

\subsubsection{Base}

The base is secured to a support structure in the anechoic chamber. It has vertical supports which are placed at precisely the outer width of the outlet chamber. By unscrewing knobs holding the scale model to the base, the scale model can be removed allowing for quick access to the cone, perforated cylinder and diffuser.

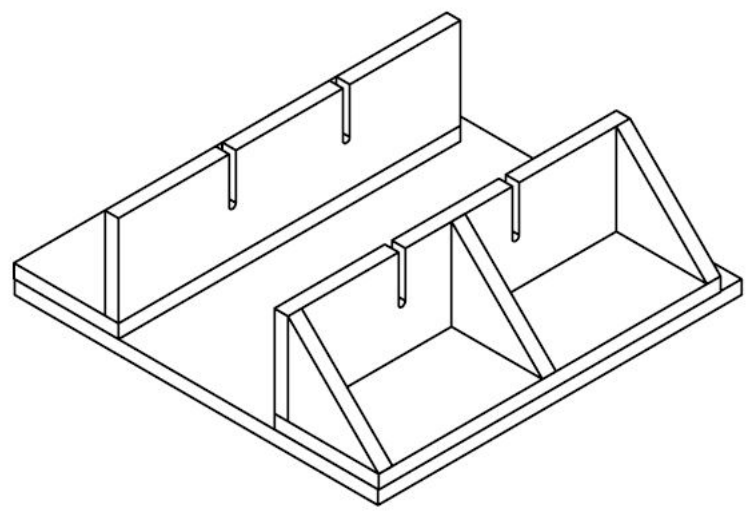

Figure 5.6: An isometric schematic of the base assembly 


\subsection{Primary Nozzle Detailed Mechanical Design}

\subsubsection{Design Overview}

The primary nozzle is made from schedule 40 steel pipe. The flange connecting to the flexible air hose is custom made from $5 / 8$ inch $(16 \mathrm{~mm})$ steel with through-holes for a bolted connection. A threaded tee adaptor is welded a short distance downstream of the flange to accommodate the total pressure probe. A plug with a concentric hole is threaded into the tee adaptor. A 0.1 inch $(2.5 \mathrm{~mm})$ outer diameter (OD), 0.07 inch (1.8 mm) inner diameter (ID) stainless steel tube with a 90 degree bend is secured in the plug with epoxy in such a way that the opening of the tube is centered in the pipe and facing into the flow. This allows for the total pressure to be measured. This tube is held within a 0.12 inch $(3.0 \mathrm{~mm})$ OD tube in order to increase the structural strength of the total pressure probe and help prevent potential vibrations.

The downstream portion of the primary nozzle, containing the flow conditioners, is made from steel schedule 40 pipe half an inch greater in diameter than the upstream portion. This step diameter increase allows the spacers in the primary nozzle to be made from leftover pipe. It also provides a shoulder against which the spacers and flow conditioners can be held. A number of spacers are cut to $0.75,1$ and 1.25 inch lengths (19, 25 and $32 \mathrm{~mm}$ respectively). This allows for a variety of spacing increments to be used and the spacing can be changed as needed. The final inch of the primary nozzle is internally threaded to accept the contraction. The threads have 16 teeth per inch and are custom cut in order to remove minimal material from the wall. 

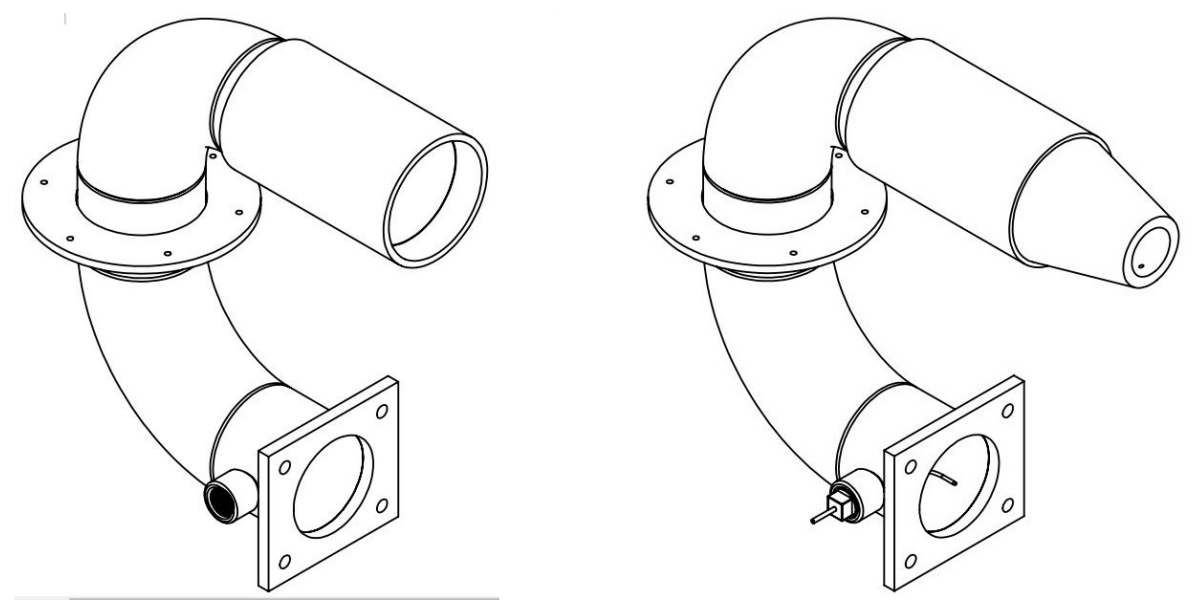

Figure 5.7: A schematic of the primary nozzle weldment (left) and the fully assembled primary nozzle (right)

\subsubsection{Primary Nozzle Stress}

An examination of the stresses acting in the primary nozzle is required to ensure safe operation. As mentioned previously the HSWT contains air at a maximum pressure of approximately 100 PSI $(689 \mathrm{kPa})$. The stress analysis is performed for a worstcase scenario of complete blockage at a full supply pressure. The method outlined in Introduction to Pipe Stress Analysis by Sam Kannappan [94] is used, providing a factor of safety on the allowable working pressure of 24 for the 3 inch section and 22 for the 3.5 inch section. In reality the primary nozzle never experiences a static pressure above approximately 12 psi thus the non worst-case scenario factor of safety is much higher. The threads and elbows may cause a lower safety factor but are not considered further. The primary nozzle was pressure tested at 90 psi (maximum shop air pressure available) before use and no leaks or failure was observed. The pressure was cycled ten times with no abnormalities noticed. 


\subsection{Scale Model General Structural Considera- tions}

The mechanical strength of the scale model is verified based on the expected forces in order to reduce or eliminate failure of any components during testing. The anticipated forces include

- The force and moment reaction at the primary nozzle mounting point caused by the thrust from the primary nozzle

- The force and moment reaction at the primary nozzle mounting point caused by the weight of the primary nozzle and flexible air hose

- Impingement forces at the cone

- Forces caused by the pressure drop of the inlet and outlet perforated plates

A force analysis is undertaken at both the nominal flow conditions and choked nozzle flow conditions to verify the model strength. The calculations are omitted for brevity, however, for the choked conditions (worst case scenario), it is found that the net reaction force and moment at the primary nozzle mounting point is $476.5 \mathrm{~N}$ (107.1 lbf) and $86.2 \mathrm{Nm}(63.5 \mathrm{ft}-\mathrm{lb})$ respectively. The impingement force at the cone is estimated at 523.9 Newtons $(117.7 \mathrm{lbf})$. The pressure drops from the inlet and outlet perforated plates are quite low thus the resulting forces are negligible.

The primary nozzle is mounted using construction adhesive and six 1.5 inch \#8 wood screws. Reference [95] finds that a single \#8 screw face screwed into 23/32 inch $(18.3 \mathrm{~mm})$ southern pine plywood requires $491 \mathrm{lbf}(2184 \mathrm{~N})$ to pull out. It is also found that $380 \mathrm{lbf}(1690 \mathrm{~N})$ is required to pull out \#8 wood screws screwed into the edge of the same plywood. Although the wood used in this project differs 
slightly, the use of these values as an estimate of the strength implies that the primary nozzle could likely be mounted with a single screw and no glue without failure. For this reason, 6 screws with glue is deemed adequate. The impingement force is easily withstood by the six $3 / 8$ inch $(9.5 \mathrm{~mm})$ elevator bolts and knobs used to secure the scale model to the base. In addition, none of the forces acting on the plywood are deemed large enough to cause a failure without some form of defect present.

\subsection{Acoustic Considerations}

The frequencies recorded in the scale model can be scaled linearly through a commonly used relationship, as presented by Kuttruff [78] among others.

$$
f_{\text {fullscale }}=\frac{1}{S . F .} \quad \frac{c_{\text {fullscale }}}{c_{\text {modelscale }}} f_{\text {modelscale }}
$$

where $f$ is the frequency and $\mathrm{c}$ is the speed of sound. By approximating the speed of sound to be the same in the model and the full scale, a simplified relation can be used:

$$
f_{\text {fullscale }}=\frac{f_{\text {modelscale }}}{S . F .}
$$

The range of interest in a full scale ejector, 0-100 Hz, scales to 0-7,059 $\mathrm{Hz}$ in the model scale. In order to capture the acoustics 10 microphone positions are used with a sampling rate of $15,000 \mathrm{~Hz}$ to meet the Nyquist sampling criteria. The placement of the microphones is relatively arbitrary but they are positioned in such a way to attempt to measure the acoustics without damaging any of the microphones. Third party computational fluid dynamics (CFD) results were used in an attempt to place the microphones in areas of low flow impingement. Figure 5.8 shows the microphone 
locations and enumeration used.

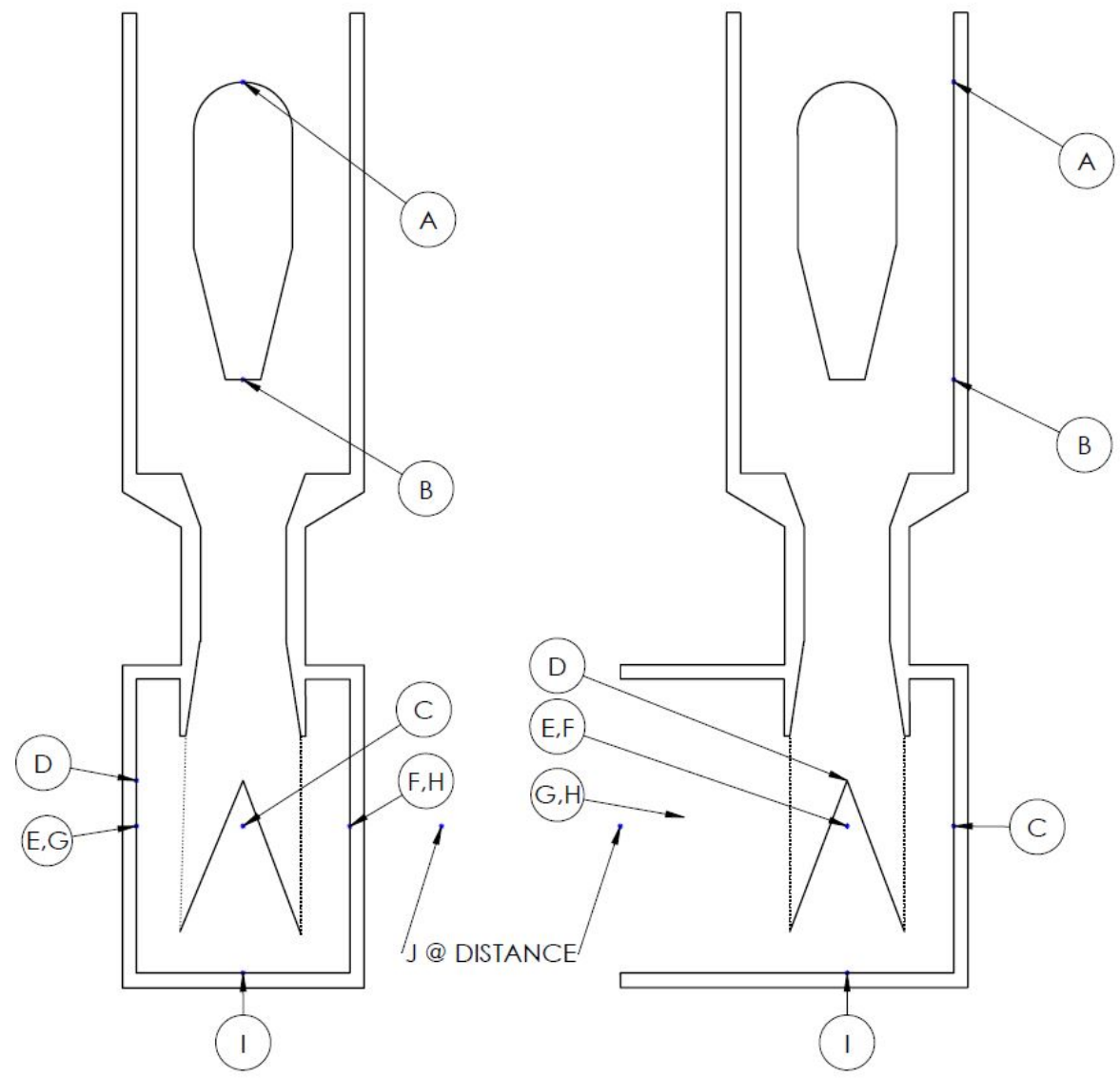

Figure 5.8: A front (left) and side (right) view schematic showing the microphone enumeration

The noise amplitudes found in the scale model could also be scaled using any number of scaling laws such as those presented by Kandula [92]. With that being said, full scale performance data (temperature, pressure, mass flow etc.) is unavailable to the author. This data is required to use the amplitude scaling laws and thus the amplitudes are not corrected in any way in this work.

Certain concerns do arise in acoustic scale models but they are often hard to address. The most prevalent is the attenuation of sound waves through air, which is a function of the frequency of the wave. The sound frequencies in the scale model are 
different than those in the full scale facility thus the attenuation of the sound waves is inevitably different. Given the relatively small distances in the scale model this is not accounted for but it should be kept in mind.

Another important consideration is the sound absorption/reflection by the construction material, that is what percentage of the sound is reflected, absorbed and transmitted upon impacting the surface. Much like attenuation in air, the sound absorption of a surface is frequency dependent. The proportion of incident sound pressure which is reflected off a given surface/material can be used to define an "absorption coefficient" for the given surface/material. The coefficient is dependent on the incidence angle thus the random-incidence sound absorption coefficient has been adopted. Further information can be found in the book Auralization by Vorlander [96]. Table 5.1 $\left.\right|^{\mid}$shows a comparison of the random-incidence sound absorbance coefficients for steel \& aluminum and wood at a range of frequencies. Although not identical, the absorption coefficients for the various materials are fairly similar and not expected to have a large impact on noise results given the high amplitudes expected.

Table 5.1: Random-Incidence Absorption Coefficients of Materials Used in Full Scale and Scale Model Facilities, from [96]

\section{Random-Incidence Absorption Coefficient}

\begin{tabular}{cccccccc} 
Material & \multicolumn{6}{c}{ Octave band frequency $(\mathrm{Hz})$} \\
& 125 & 250 & 500 & 1000 & 2000 & 4000 & 8000 \\
\hline Steel \& Aluminum & 0.02 & 0.02 & 0.03 & 0.03 & 0.04 & 0.05 & 0.05 \\
Wood & 0.18 & 0.12 & 0.10 & 0.09 & 0.08 & 0.07 & 0.07
\end{tabular}

\footnotetext{
${ }^{1}$ Note that the values for steel and aluminum are approximated from "walls, hard surfaces average" and the values for the $1 / 2$ inch $(12.7 \mathrm{~mm})$ plywood used are approximated by "16 $\mathrm{mm}$ wood on 40 mm studs".
} 


\subsection{Scale Model Aerodynamic Design}

Only a small portion of the scale model requires aerodynamic design given the matching of geometric similarity. Due to the scale factor, the primary nozzle size is constrained, however, the flow conditioning of the primary nozzle requires complete design thus a detailed aerodynamic calculation is undertaken. This calculation is done as a design stage verification to ensure that the wind tunnel is capable of providing the required mass flow rate and pressure to reach the desired velocities. In addition to the overall design of the primary nozzle, each individual flow conditioning component also needs to be designed. The same is true for the pressure probes and the outlet chamber perforated plate. The following sections summarize the aerodynamic design of the individual components of the scale model.

\subsection{Primary Nozzle Aerodynamic Design}

An iterative Excel spreadsheet is used to solve all primary nozzle flow parameters isentropically, including temperature, pressure, velocity and Mach number to name few. The analysis begins at the nozzle outlet with assumed atmospheric conditions and works its way backwards to the flanged connection taking the pressure drops of each component into consideration. The $3^{\text {rd }}$ edition of the book Handbook of Hydraulic Resistance, by I.E Idelchik [23] is used to estimate the pressure drop of each component of the primary nozzle. Where there is any doubt or where two methods are possible for calculating a pressure drop, the more conservative value (greater pressure drop) is used. For nominal flow conditions the primary nozzle total pressure loss is estimated at 3.8 psi $(26,476 \mathrm{~Pa})$. For the choked nozzle flow condition, the total pressure loss is estimated to be $4.6 \mathrm{psi}(31,785 \mathrm{~Pa})$. The flexible hose and valve also cause a pressure drop, however, these components are required in the setup thus their 
pressure loss is unavoidable and cannot be designed for. The primary nozzle used is a single iteration design, however, improvements could be made through further experimentation.

The large change in temperature caused by the air expansion in the tanks and from the flow acceleration may impact the aerodynamics thus it is investigated. The total temperature is varied in the iterative calculation from atmospheric conditions $\left(+20^{\circ} \mathrm{C}\right)$ to a low value $\left(-20^{\circ} \mathrm{C}\right)$ estimated to be the lowest possible temperature experienced in the scale model (estimated based on preliminary results of Giardino). It is found that certain variables undergo significant changes, such as the density, speed of sound, mass flow rate and velocity. Certain other variables such as the Mach number, dynamic pressure, pressure loss from each component and primary nozzle pressure ratio remain constant. The control variables remain constant therefore no temperature based compensation is made to the control system or to the results. Unexplained variations in the acoustics throughout a given run may be attributable to the temperature drop and should be kept in mind.

\subsubsection{Flow Straightening}

Due to the configuration of the anechoic chamber, the primary nozzle requires two $90^{\circ}$ bends to turn the flow $180^{\circ}$. These bends cause secondary flows and velocity distortions downstream of the bends as shown schematically in figure 5.9 , The establishment of secondary flows and non-uniform velocity profiles downstream of pipe bends is well established. It is however difficult to find information pertaining to the specific situation encountered by the primary nozzle, that is two in plane 90 degree

bends of a given radius with a short separation distance at a given flow speed. In addition, the unknown inlet conditions to the primary nozzle provide no information 

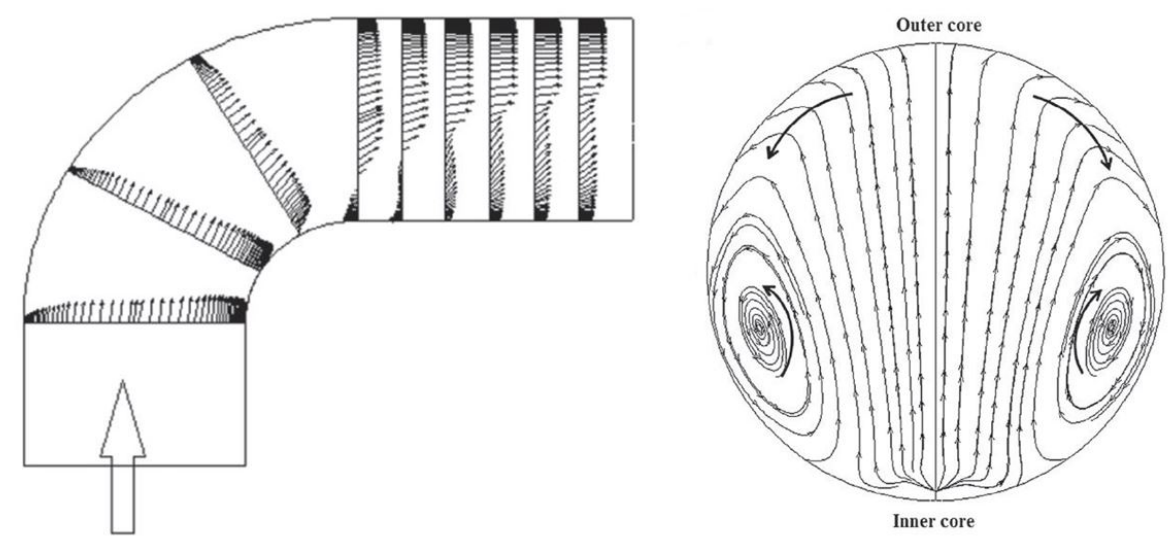

Figure 5.9: Schematic showing the velocity profile downstream of a pipe bend (left) and the secondary flows established at the outlet plane of the bend (right), adapted from [97]

on turbulence, level of swirl or other flow properties. In an attempt to improve the uniformity of the flow, flow conditioning devices are placed inside the primary nozzle. The high modularity of the primary nozzle allows them to be changed, repositioned or removed if need be.

Literature provides certain guidelines and experimental results on the use of flow conditioners, however, there is no guarantee that the chosen flow conditioners are the best option. The solution adopted works and provides adequate results thus is deemed suitable for the application. In the order encountered by the flow, the conditioners consist of:

- A perforated plate

- A honeycomb

- A screen

The following three sub-subsections outline some observations from literature pertaining to the design of flow conditioners and also outlines the design choices leading to the selection of each component. 


\section{Perforated Plate Selection}

Reference [98] shows that a choked perforated plate flow conditioner generates significantly more noise than unchoked flow conditioners and that smaller perforations generate less "self noise" than large perforations. These findings are confirmed by reference [99] and in addition, it is found that large open area ratios also tend not to attenuate upstream noise as well as smaller open area ratios.

For the flow in the primary nozzle, it is found that a plate with approximately $25 \%$ open area ratio would cause a flow speed of Mach 0.8 through the pores and this is set as the upper velocity limit to avoid choking. Through a study of literature it is found that flow conditioner plates generally have an open area ratio no greater than $60 \%$. Midway between these two, or $42.5 \%$ open area ratio is deemed a reasonable flow conditioner with many studies from literature using plates in this open area ratio range. $42.5 \%$ open area ratio plate is not commercially available thus $40 \%$ open area ratio plate is used. The commonly available pore size for $40 \%$ open area ratio is 0.125 inches or 0.25 inches $(3.2 \mathrm{~mm}$ or $6.4 \mathrm{~mm}$ respectively). In an attempt to attenuate any upstream noise (valve noise) and to reduce self generated noise, the smaller hole size is chosen.

The velocity profile downstream of a bend shows a non-uniformity, however, this velocity does tend to recover at a sufficient distance. The pressure drop from a perforated plate is a function of the dynamic pressure (velocity) and thus it is not uniform across the plate. This may lead to the condition where a velocity recovery downstream of the flow conditioner causes a velocity distortion essentially making conditions worst. This may motivate the use of a non-uniformly perforated plate, and an attempt at this is made by reference [100]. With an increasing porosity near the bend interior it is found that the velocity distortion is actually over-corrected. Selection of the proper non-uniform porosity would require experimentation and for 
this reason a uniformly perforated plate is used.

\section{Honeycomb Selection}

A honeycomb is placed downstream of the perforated plate in an attempt to reduce swirl in the flow. Reference [101] provides design rules for small wind tunnels, which the primary nozzle may be regarded as. It is stated that honeycombs help in reducing swirl and lateral velocity components up to $10^{\circ}$. Lateral components of turbulence are inhibited by the honeycomb cells and almost completely annihilated in a length of approximately 5-10 cell diameters. The optimum cell length is said to be 6-8 cell diameters and the cell size should be chosen such that the cell size is approximately one $150^{\text {th }}$ of the settling chamber diameter. Finally, it is also mentioned that the shed turbulence is greater for a honeycomb in laminar flow.

For the internal nozzle diameter of 3.068 inches $(77.93 \mathrm{~mm})$ this equates to a 0.020 inch $(0.519 \mathrm{~mm})$ cell with a length approximately 0.14 inches $(3.6 \mathrm{~mm})$. Unfortunately this size honeycomb is difficult to procure. A donation ${ }^{2}$ of $5 / 8$ inch $(15.9 \mathrm{~mm})$ thick aluminum honeycomb with $1 / 8$ inch cells $(3.2 \mathrm{~mm})$ was made to the research group thus this is used for flow conditioning. In order to more closely match the 6-8 lengthto-cell size ratio, two honeycombs are placed together giving a length to diameter ratio of 10 . The Reynolds number in this honeycomb can be estimated using the formula adapted from reference [102]:

$$
R_{e, c e l l}=\frac{\rho u_{c e l l} d_{c e l l}}{\mu}
$$

Where the hydraulic diameter for a hexagon is used:

\footnotetext{
${ }^{2}$ Thank you Steven!
} 


$$
d_{h, \text { hexagon }}=\frac{4 A}{p}
$$

Where $A$ is the cross sectional area of the cell and $p$ is the cell perimeter. This results in a cell Reynolds number of close to 20,000 which is well above the transition to turbulence value of 4000 thus the shed turbulence should be reduced.

\section{Screen Selection}

As a final step to flow conditioning, a screen is placed downstream of the honeycombs in an attempt to suppress any remaining turbulence. Reference [101] once again provides design rules for screen selection in wind tunnels. It is explained that a large pressure drop provides a greater reduction in turbulence. For a given open area ratio (OAR), a screen with a smaller mesh is better at removing pre-existing turbulence. Another important design rule is that the final screen in a wind tunnel should have an OAR greater than $57 \%$ since smaller OARs tend to produce instabilities (however, an OAR much greater than $57 \%$ should also be avoided as a lower pressure drop provides less turbulence reduction [103]). Reference [104] explains that the wire diameter should be selected such that the wire diameter Reynolds number is less than 50 to avoid vortex shedding, however, reference [103] uses screens with wire diameter Reynolds numbers above $50(\mathrm{Re}=103-330)$ and finds that the shed vortices are relatively unimportant given their high frequency and rapid rate of decay. For the screens tested (1, 10 and 34 wire mesh) it is found that 34 wire mesh provides the greatest turbulence reduction and that using more screens provides a greater flow uniformity at the cost of a greater pressure drop. This finding is echoed through literature with mesh sizes that range from about 15-42 with the higher end providing a better turbulence decrease. The wire diameters are found to range from approximately $0.25 \mathrm{~mm}$ 
to $1.5 \mathrm{~mm}$ with the majority closer to the former. Using these findings as a guideline, a stainless-steel screen with a $65 \%$ OAR, wire diameter of $0.2559 \mathrm{~mm}$ and 30 mesh is chosen.

\section{Flow Conditioner Spacing}

An important consideration in the use of flow conditioners is the spacing between conditioners. The ideal distances vary based on parameters such as flow velocity and upstream turbulence. A short survey of literature is undertaken in an attempt to place the flow conditioners at appropriate distances. A full investigation is beyond the scope of this work.

Reference [105] finds that for the turbulent case the pressure drop of a perforated plate is fully achieved 5-6 perforate hole diameters downstream and the results of Spearman [106] show that 6 pipe diameters downstream the flow is within $5 \%$ of a fully developped flow.

Reference [103] provides what could be seen as rules of thumb for component spacing. It is stated that for a honeycomb a distance of about 50 cell diameters or 1-10 cell lengths is required for turbulence decay.

The placement of the screen is guided by both references [101] and [103] with the former stating that an optimum distance between the last screen and the settling chamber contraction is 0.2 settling chamber diameters. A shorter distance leads to significant flow distortions and a longer distance leads to unnecessary boundary layer growth. The rules of thumb of [103] mention that turbulence takes 50-75 mesh lengths or 330-550 wire diameters downstream to decay.

Summarizing these rules, the honeycomb should be placed between 5-6 perforate hole diameters (0.625-0.75 inches or 15.9 to $19.1 \mathrm{~mm})$ and 6 pipe diameters (approximately 15 inches or $381 \mathrm{~mm}$ ) downstream of the perforated plate. The screen should 
be placed 50 cell diameters $(6.25$ inches or $158.75 \mathrm{~mm})$ or 1-10 cell lengths (1.25-12.5 inches or 31.8-317.5 mm) downstream of the honeycomb. The contraction should be placed 50-75 mesh lengths (1.66-2.5 inches or 42.2-63.5 mm) or 330-550 wire diameters (2.145-3.25 inches or 54.5-82.6 mm) downstream from the final screen.

Unfortunately, space in the secondary flow chamber (thus primary nozzle total length) is limited and these distances cannot all be respected. For these reasons the perforated plate is placed against the back shoulder inside the primary nozzle, approximately 0.5 inches $(12.7 \mathrm{~mm})$ from the exit plane of the final bend. The honeycomb is placed 1 inch $(25.4 \mathrm{~mm})$ downstream of the perforated plate with the screen placed 1.75 inches $(44.5 \mathrm{~mm})$ downstream of the honeycomb. The distance from the screen to the contraction is also 1.75 inches $(44.5 \mathrm{~mm})$. These dimensions equate to the honeycomb being 8 perforate diameters or 0.3259 settling chamber diameters downstream of the perforated plate, the screen being 14 honeycomb cell diameters or 1.4 cell lengths downstream of the honeycomb and the contraction being 53 mesh lengths or 174 wire diameters downstream of the screen. These are not ideal spacings but rather those available within the limited space of the primary nozzle and secondary flow chamber.

\subsubsection{Primary Nozzle Pressure Ratio}

As mentioned previously, the primary nozzle pressure ratio is required for velocity control thus total and static pressure probes are used to obtain this pressure ratio. Care is taken to design accurate probes based on information available in literature.

\section{Total Pressure Probe Design}

Reference [107] states that the total, or impact, pressure can be conveyed by almost any size and form of nozzle provided it squarely faces the wind in the centerline of 
a pipe. In addition, it is shown that calibration corrections for square ended total pressure tubes is relatively constant, at a value near 1.0, for Reynolds numbers greater than 100 (based on outer diameter where the internal diameter is approximately 0.74 of the outer diameter). For this reason the total pressure tube inner diameter is chosen to be 0.071 inches $(1.8 \mathrm{~mm})$ such that the Reynolds number is approximately 400, or 4 times larger than the inflection point of 100 .

Reference [107] also provides information on the effect of the total pressure probe opening shape and yaw angle, as shown in figure 5.10 .

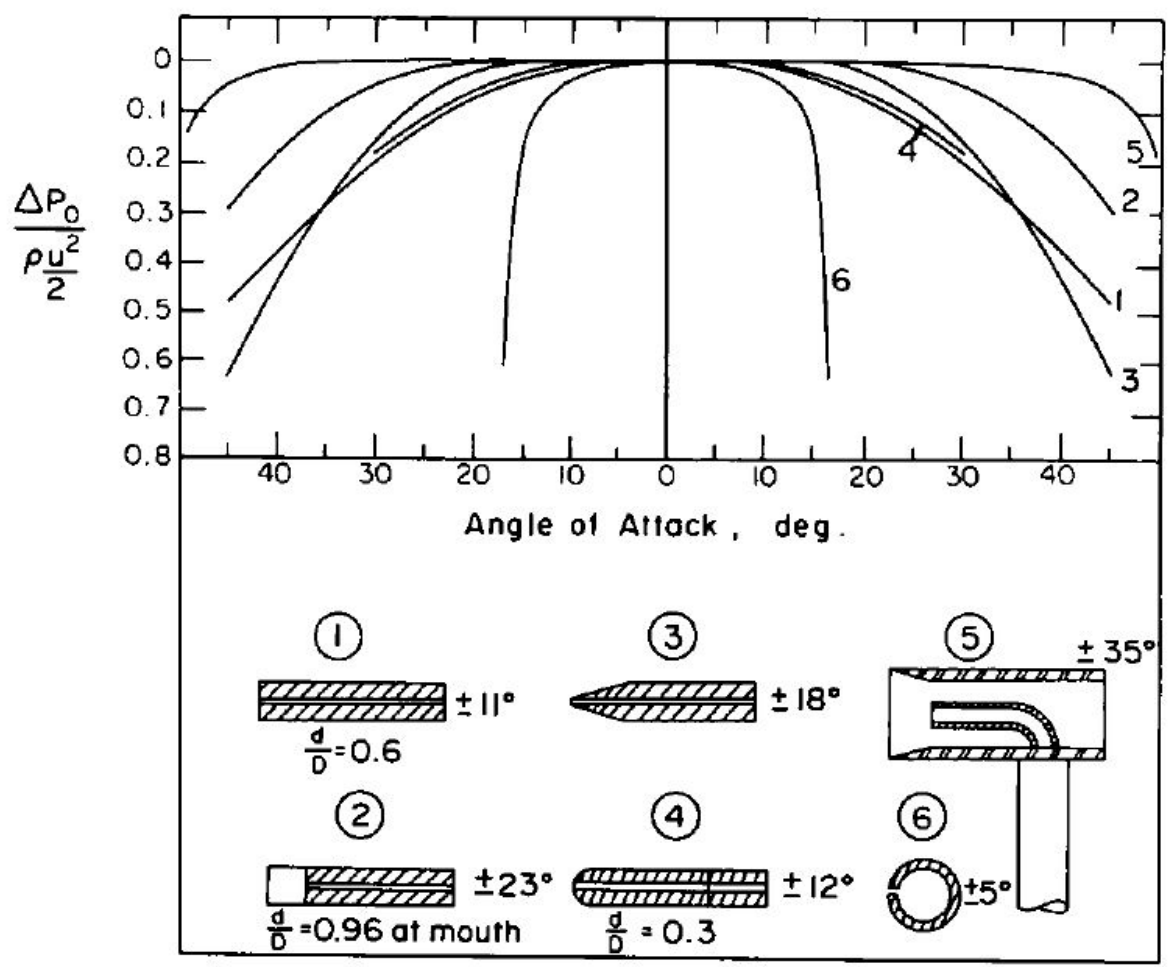

Figure 5.10: Schematic showing the impact of probe tip shape and yaw angle, from [107]

As can be seen for low yaw angles (within approximately $\pm 10^{\circ}$ ) the end shape has no influence. For the total pressure tube used in this research the end is kept square to simplify manufacturing. The opening is placed square to the flow to avoid 
any yaw effects and it is believed to be square to within a few degrees. The book Springer Handbook of Experimental Fluid Mechanics [108] describes the error which can be encountered when trying to measure the total pressure of a non-homogenous flow with a pitot probe which is too large. In this case the diameter of the probe is deemed small enough that any flow inhomogeneity seen at the total pressure probe will be small and the resulting error negligible. The total pressure probe is centered in the pipe in an attempt to measure the maximal flow velocity and to reduce any wall proximity effects. The tip of the probe extends multiple probe stem diameters downstream from the support stem to eliminate any influence that the support stem may have on the flow.

\section{Static Port Design}

The design of a static pressure measurement port follows many of the same rules as for a total pressure tube. An important consideration for static pressure ports flush to the surface is the requirement to have a burr-free hole (reference [107]) and for the hole to be of a size small enough to avoid cavity vortices (reference [109]). Reference [109] points out that the length (depth) of the port hole should be at least twice as large as the port diameter such that the flow structure within the port is fully developed and not changing with flow Reynolds number. In addition, it is discussed that a static port diameter smaller than 0.1 pipe diameters (pipe being the secondary flow chamber in this case) provides no appreciable change in the flow structure of the main flow near the static port.

Unfortunately the primary nozzle provides no suitable location for a static pressure tap. For this reason the static pressure is measured at the wall of the secondary flow chamber. Third party CFD results show that the static pressure at this location is nearly identical to that in the jet, following the expected trend from literature. 
Small tubes of 0.047 inch $(1.19 \mathrm{~mm})$ inner diameter with 0.009 inch $(0.229 \mathrm{~mm})$ wall thickness are available to the author thus these are used for static pressure taps. These long tubes are cut to a length many times longer than the tube diameter to greatly surpass the length to diameter ratio and they are meticulously prepared to have a chamfer free, burr free end which is square. The resulting tubes are then inserted into a small hole drilled in the location of interest and held in place with epoxy. Care is taken to ensure the tap is flush to the wall. Although this setup may not be perfect, it is later calibrated using a Pitot-static probe thus small errors are ultimately not of large consequence.

\subsection{Inlet and Outlet Perforated Plate Selection}

The scale model inlet perforated plate is chosen to match the geometry used in third party scale model tests. The outlet perforated plate is chosen such that its pressure drop is equivalent to that used in third party scale model tests. From pitot-static traverse measurements, the third party total pressure and velocity are found directly below the plate. From this data, a $58 \%$ OAR is chosen as it provides a conservative pressure drop (one slightly smaller than the actual pressure drop as this allows for holes in the perforated sheet to be selectively blocked, decreasing the open area ratio thus increasing the pressure drop). 


\section{Chapter 6}

\section{Jet Velocity Control and Calibration}

Before commencing acoustic tests and characterization, considerable effort was required to attain proper primary nozzle velocity control. This chapter outlines the tests performed and modifications made to the control system in order to obtain acceptable velocity control and presents the primary nozzle calibrations. The modifications undertaken are presented chronologically with the intent of being a good reference for future users of the facility.

\subsection{Data Analysis Procedure}

The data obtained for this chapter comes solely from the pressure measurement system. The data is analyzed using Microsoft Excel to observe trends in time, calculate averages and do other basic analysis'. As mentioned previously, the data is recorded at a sampling rate of $2000 \mathrm{~Hz}$ with an averaging of 200 samples. The data recorded during every run is:

1. The time, beginning at 0 for every new run

2. The Control Mach Number, calculated isentropically in LabVIEW from equation 4.2 and items 3 and 4 
3. The absolute total pressure, calculated in LabVIEW using the user input atmospheric pressure and item 9 or $10^{1}$

4. The absolute static pressure, calculated in LabVIEW using the user input atmospheric pressure and item 7 or $8^{2}$

5. The control valve voltage input

6. The absolute atmospheric/chamber pressure, absolute pressure recorded at the beginning of each day and chamber pressure monitored throughout each run, calculated in LabVIEW using the appropriate transducer calibration constants

7. Primary nozzle differential static pressure, calculated in LabVIEW using the appropriate transducer calibration constants

8. Pitot-static probe differential static pressure, calculated in LabVIEW using the appropriate transducer calibration constants

9. Pitot-static total differential pressure, calculated in LabVIEW using the appropriate transducer calibration constants

10. Primary nozzle total differential pressure, calculated in LabVIEW using the appropriate transducer calibration constants

For primary nozzle calibration the following data is recorded in addition to the above.

11. The Pitot-static probe Mach number calculated isentropically in LabVIEW (whether used for control or not)

\footnotetext{
${ }^{1}$ Item used is that which is used for control

${ }^{2}$ see footnote 1
} 
12. The calibrated nozzle total pressure, calculated in LabVIEW using the appropriate transducer calibration constants and nozzle total pressure calibration polynomial

13. The calibrated nozzle static pressure, calculated in LabVIEW using the appropriate transducer calibration constants and nozzle static pressure calibration polynomial

\subsection{Baseline Performance}

The first runs of the ejector are performed using the baseline parameters for the auxiliary flow path as suggested by Hall, that is a proportional gain $K_{P}$ of 4 , an integral gain $K_{I}$ of 1 , a derivative gain $K_{D}$ of 1 , a valve slack of 3.5 volts, a deadband compensation of 3 volts and no undershoot correction. The run time is set to 120 seconds but it is cut short for runs where the performance is poor or pressures exceed the transducer limits. Figure 6.1 shows the primary nozzle Mach number performance for the baseline parameters as measured using the Pitot-static probe in the primary nozzle potential core. It should be noted that in this figure, as for all other figures with Mach 1 velocity, the validity of Mach 1 should not be taken certainly as it is isentropically calculated from the pressure ratio without compensation for any shock waves or other sonic flow phenomena.

As is easily apparent, the resulting performance is unacceptable as there are large Mach number variations in the steady state. An attempt to obtain better performance was undertaken without any specific target, but rather a "best possible" objective. 


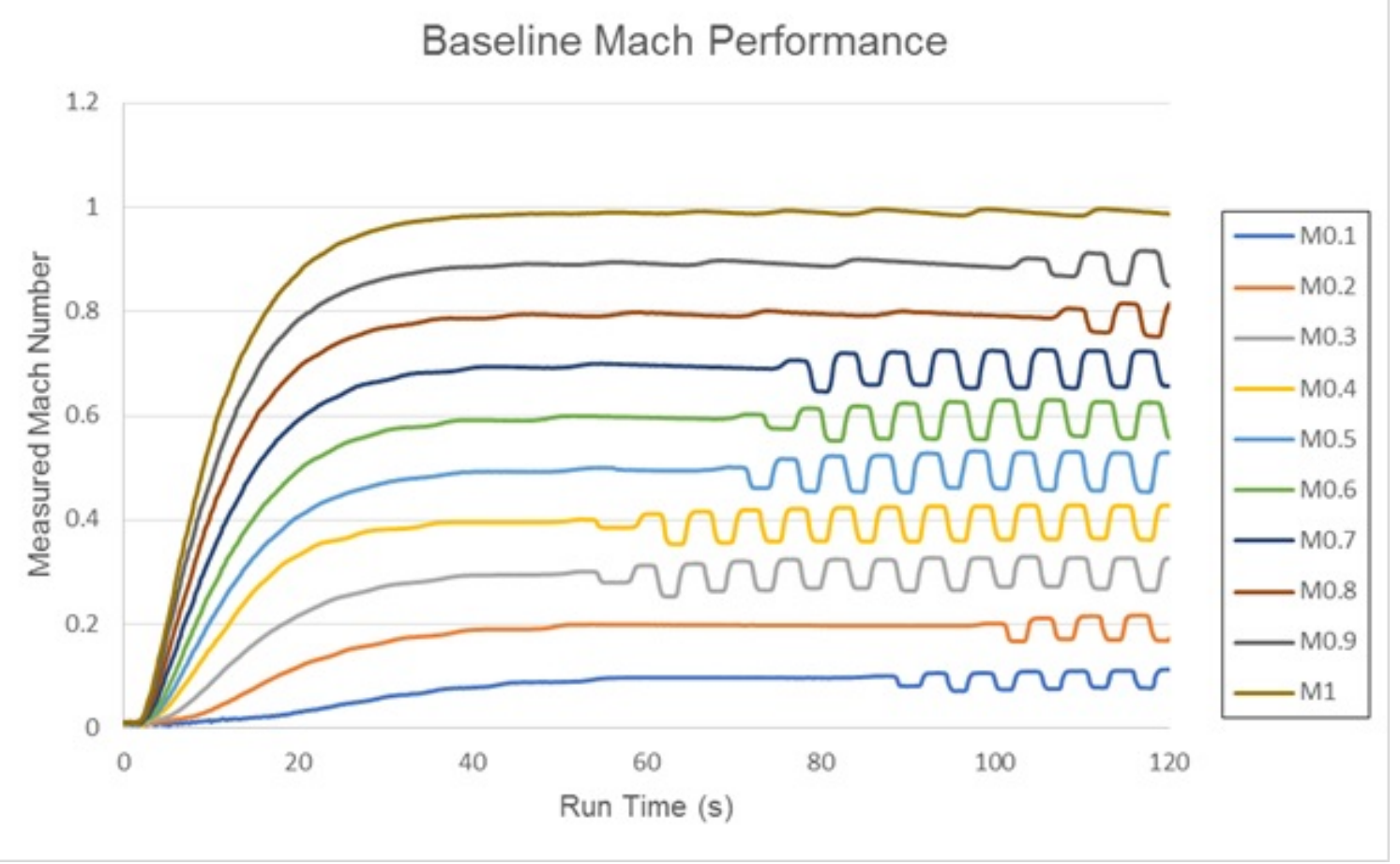

Figure 6.1: Baseline Mach performance of the primary nozzle with the parameters suggested by Hall

\subsection{Deadband Compensator Off}

The large fluctuations are believed to originate from the deadband compensator, therefore, tests are performed with the deadband compensation off (deadband compensation $=0$ volts $)$ with figure 6.2 showing the steady state result, which display a reverse sawtooth pattern. This sawtooth pattern is not acceptable due to the magnitude of the oscillations. The reverse sawtooth pattern was described by Hall and believed to originate from the valve deadband.

The deadband is an effect similar to (but different than) hysteresis where a supply voltage sent to the valve has no effect for a given time or voltage increase, normally when the valve changes opening direction. The exact mechanism behind this effect 


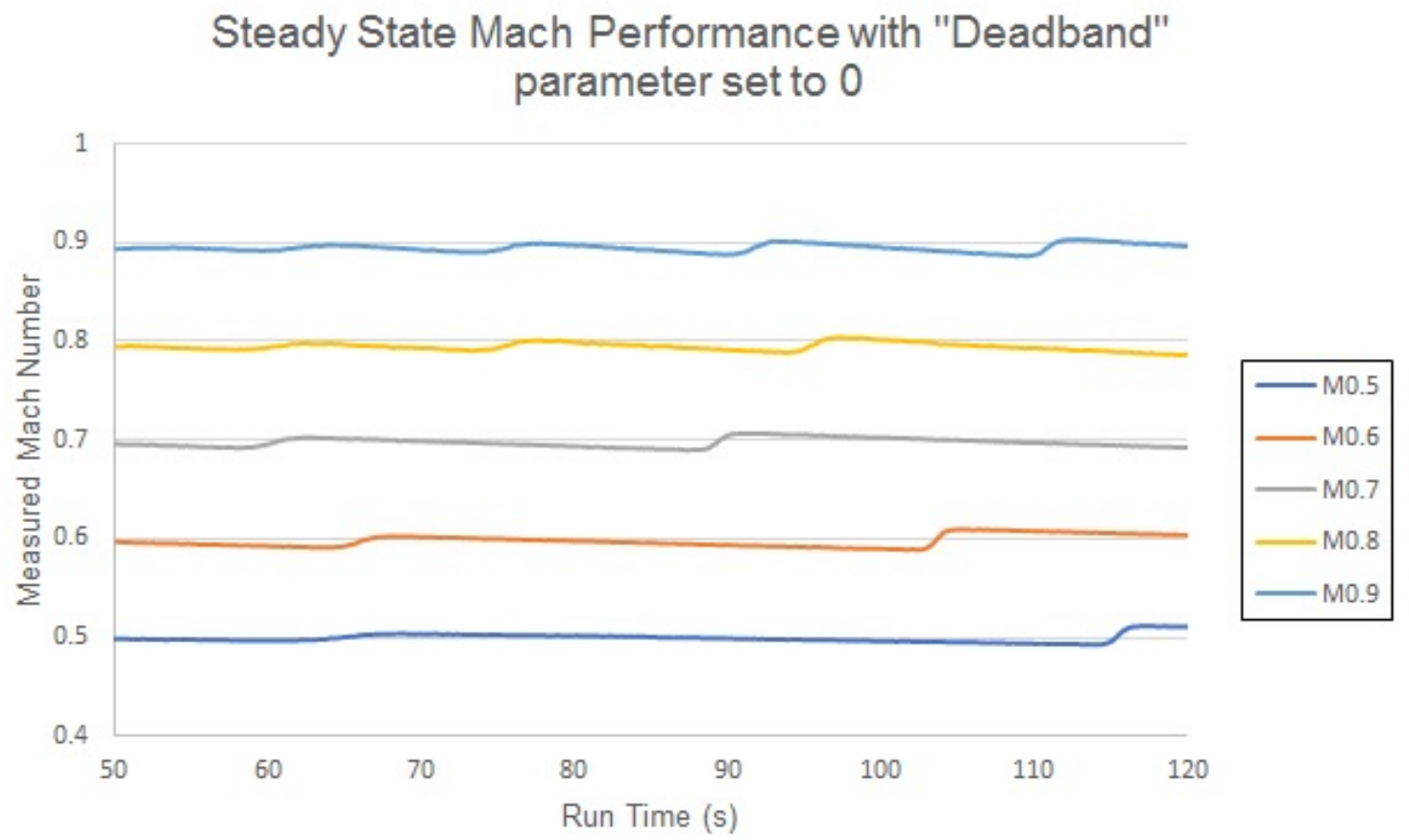

Figure 6.2: Steady state Mach performance of the primary nozzle with baseline parameters and the deadband set to zero

is unknown ${ }^{3}$, however, it is known that after a given time, or increase in voltage, the valve has traversed the deadband and opens in a step manner normally overshooting the desired or target valve position. The valve then tries to close to counter this overshoot and experiences a deadband in the opposite direction. The target valve position continuously changes because of the decreasing tank pressure and in many cases this leads to a situation where the target valve position crosses the actual valve position faster than the valve can get through the deadband. This causes the valve to change opening directions, going through the deadband once again.

\footnotetext{
${ }^{3}$ This is generally not believed to be due to lack of lubrication or other improper maintenance
} 


\subsection{Deadband Compensator Variations}

In an attempt to obtain better performance from the deadband compensator, variations in the compensation value are tested. All tests are performed with a target Mach of $0.85, K_{P}=10, K_{I}=1$ and $K_{D}=1$. Mach 0.85 is chosen as it is close to the

design Mach number $4^{4}$ and the gain values are arbitrarily chosen. The results for different settings of the deadband compensator are shown in figure 6.3 .

\footnotetext{
${ }^{4}$ Exact value omitted to protect intellectual property
} 

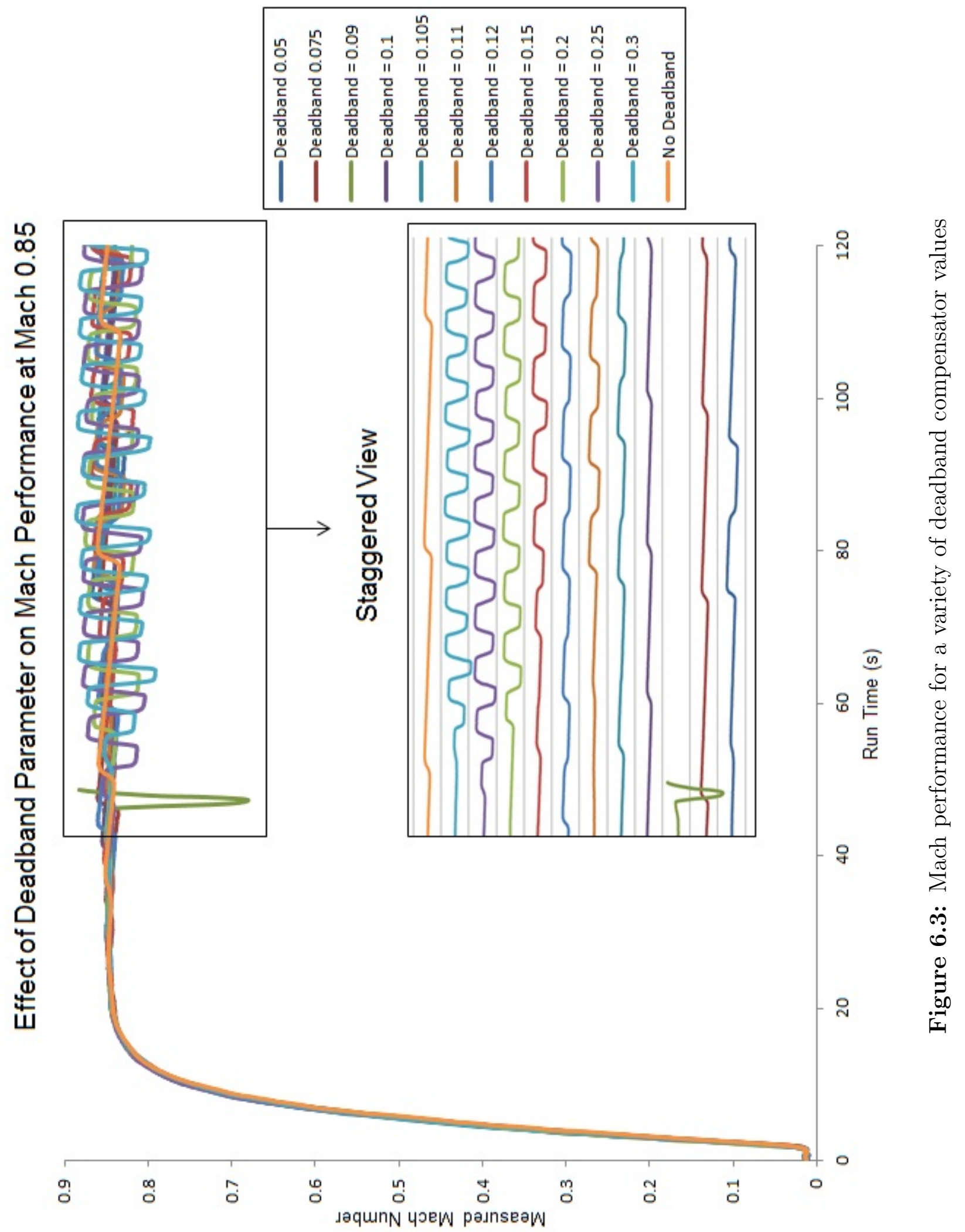
As can be seen, the compensator appears to do nothing (deadband $=0.1$ ), or to overcompensate (deadband $=0.105$ ) with no middle ground. For none of the tested values does the deadband compensator provide any benefits. It is unknown why the deadband compensator is performing poorly thus it is set to 0 for the remainder of this work.

\subsection{Valve Voltage Analysis}

In an attempt to better understand the deadband phenomenon a long duration run was undertaken at Mach 0.85 with the same gains. The resulting Mach number is plotted along with the valve supply voltage in figure 6.4. A steady-state portion of this plot is shown in detail in figure 6.5. This extended run also serves the purpose of providing an estimate of the maximum run time. The test ran for 707 seconds (11 minutes 47 seconds) at which point the valve supply voltage maxed out, stopping the control program.

In an ideal world the voltage would slowly increase and the valve would follow suite with no deadband. As can be seen in figure 6.5 the voltage both increases and decreases due to the valve overshoot. It can also be seen that as the Mach number crosses the target Mach number (i.e. once the target valve position crosses the actual valve position) the voltage reaches a local minimum and begins to increase, causing the valve to traverse the deadband once again. Figure 6.6 shows the first derivative of the supply voltage, obtained using the center difference method, where the correlation between the valve voltage and the reverse sawtooth pattern Mach number is apparent. The derivative of the voltage is minimum after the valve shoots open, is zero when the Mach number crosses the target value and is maximum just before the valve 


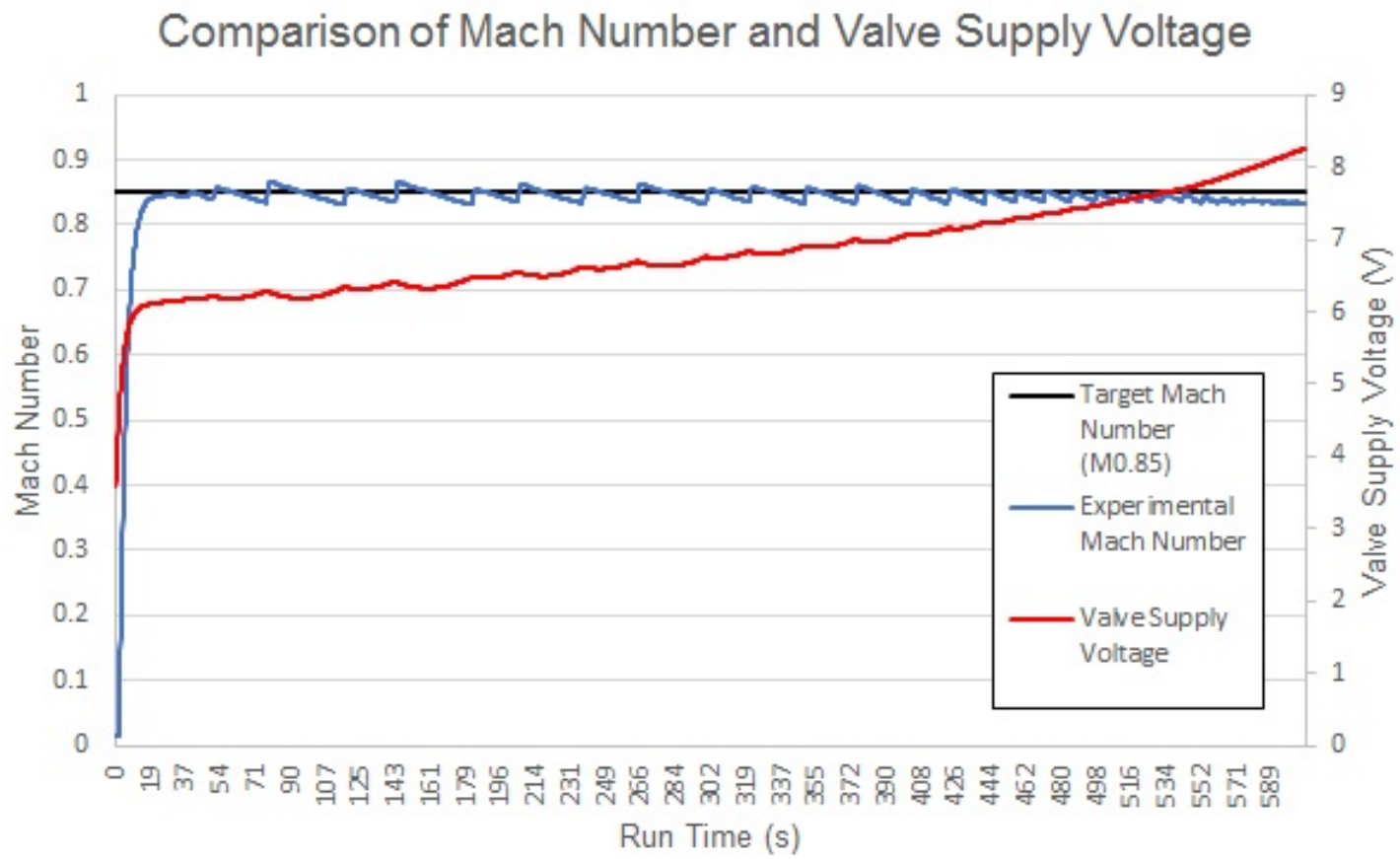

Figure 6.4: Comparison of the Mach performance and valve supply voltage for a long duration run

overshoots, as expected.

From experiments it appears as though the Mach number sawtooth amplitude and phase depend on many parameters including the tank pressure, target Mach number and control gains. For consistency, all test runs are started when the tanks are at 90 psi $(620 \mathrm{kPa})$ with the compressor not in standby. Several fixes to the deadband problem were attempted and these include:

- Limiting the valve supply voltage to increases only, in order to prevent the valve from going through alternating forward-backwards deadbands

- Supplying a sinusoidal voltage so the valve is constantly working through the deadband and ready to respond in either direction

- Applying a step voltage input every time the derivative of the supply voltage 


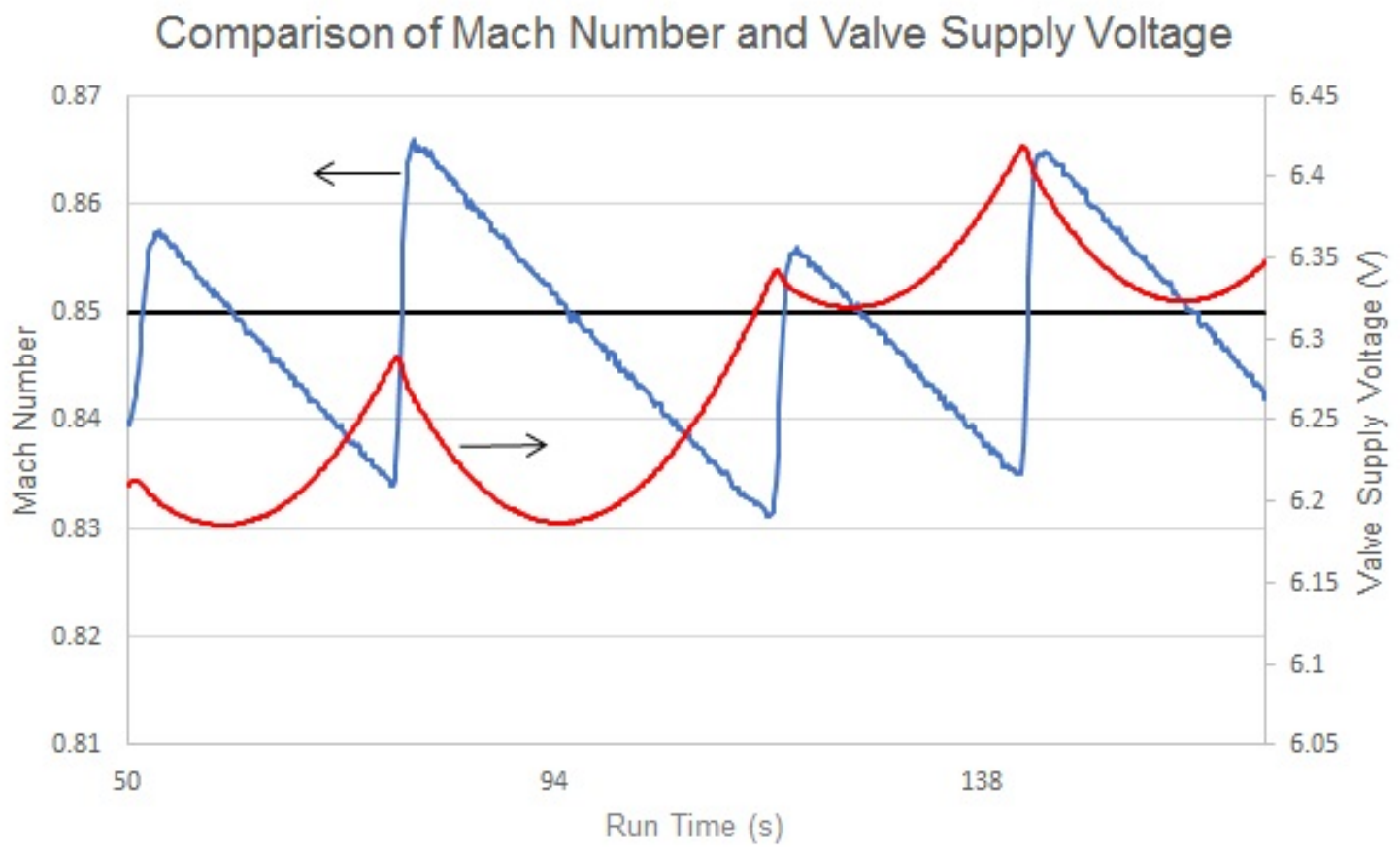

Figure 6.5: A portion of the comparison of the Mach performance and valve supply voltage

equals zero

None of these solutions worked - mostly due to the valve response time being too slow - thus alternate solutions are explored

\subsection{Varying Valve Supply Pressure}

Given that the valves are electro-pneumatic they are supplied with shop air. The valves require a pressure ranging between 60-100 psi (414-689 $\mathrm{kPa})$. An attempt at varying the supply pressure to investigate its impact on the sawtooth pattern is undertaken. The reasoning is to attempt to help the valve overcome any stiction by increasing the shop air pressure thus the internal forces in the valve. The results can be seen in figure 6.7 where the test is performed with the same conditions as 


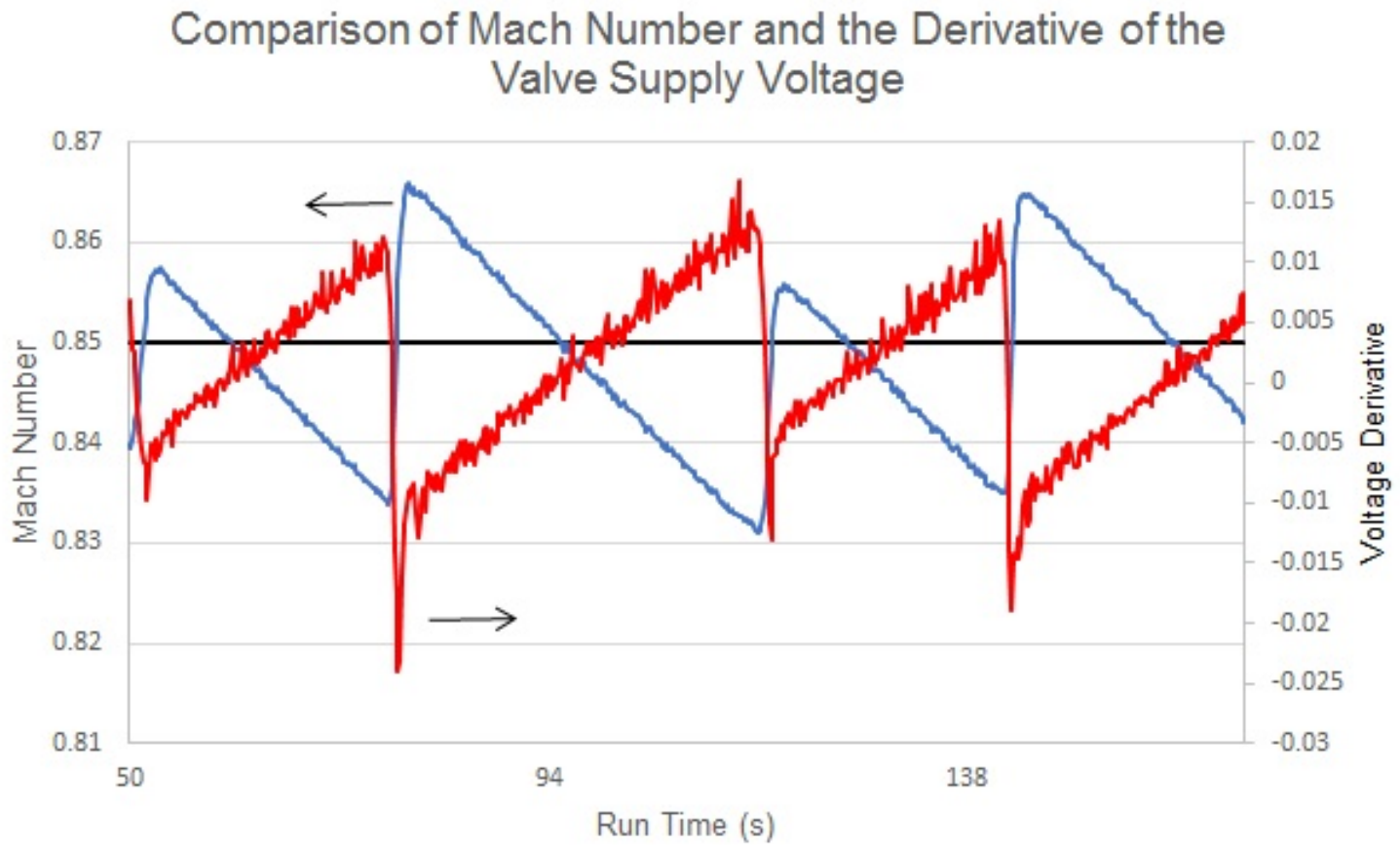

Figure 6.6: A portion of the comparison of the Mach performance and derivative of the valve supply voltage

previously but with the deadband compensator off. It can be seen that the nature of the sawtooth changes, however, it is present and of similar amplitude for all supply air pressures tested, therefore, the shop pressure is kept nominal for the remainder of this work.

\subsection{PID Tuning}

As mentioned previously, the reverse sawtooth pattern varies based on the PID gains used, with larger $K_{P}$ values increasing the magnitude of oscillations. A lengthy investigation of the impact of different gain values on the steady state performance is undertaken. The results are divided into "large" gain values and "small" gain values, with large and small being relative to this system. 
Effect of Control Valve Supply Pressure on Mach performance $(\mathrm{Kp}=10, \mathrm{Ki}=1, \mathrm{Kd}=1)$

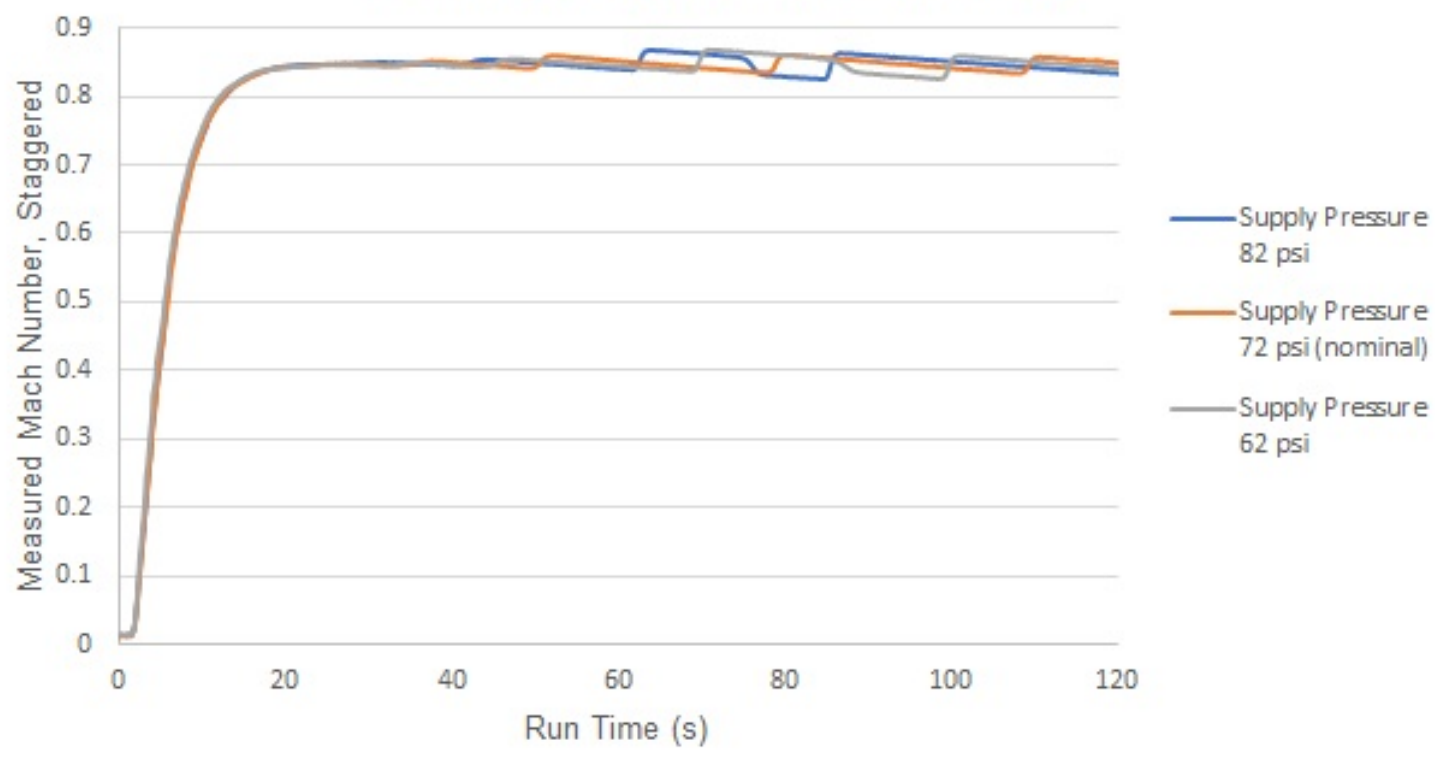

Figure 6.7: Impact of varying the supply pressure to the electro-pneumatic control valve on the Mach number unsteadiness

\subsection{1 "Large" Gain Values}

12 different combinations of "large" PID gains were tested at Mach 0.85 with the results shown in figure 6.8, . 


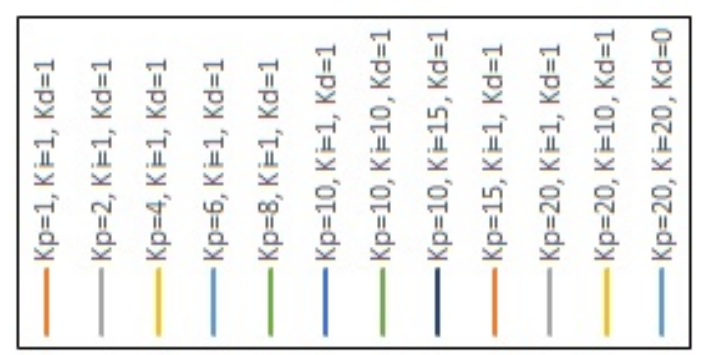

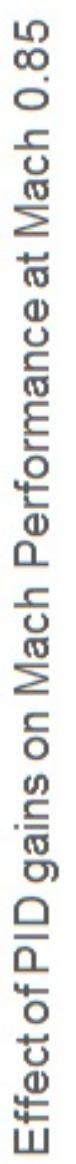
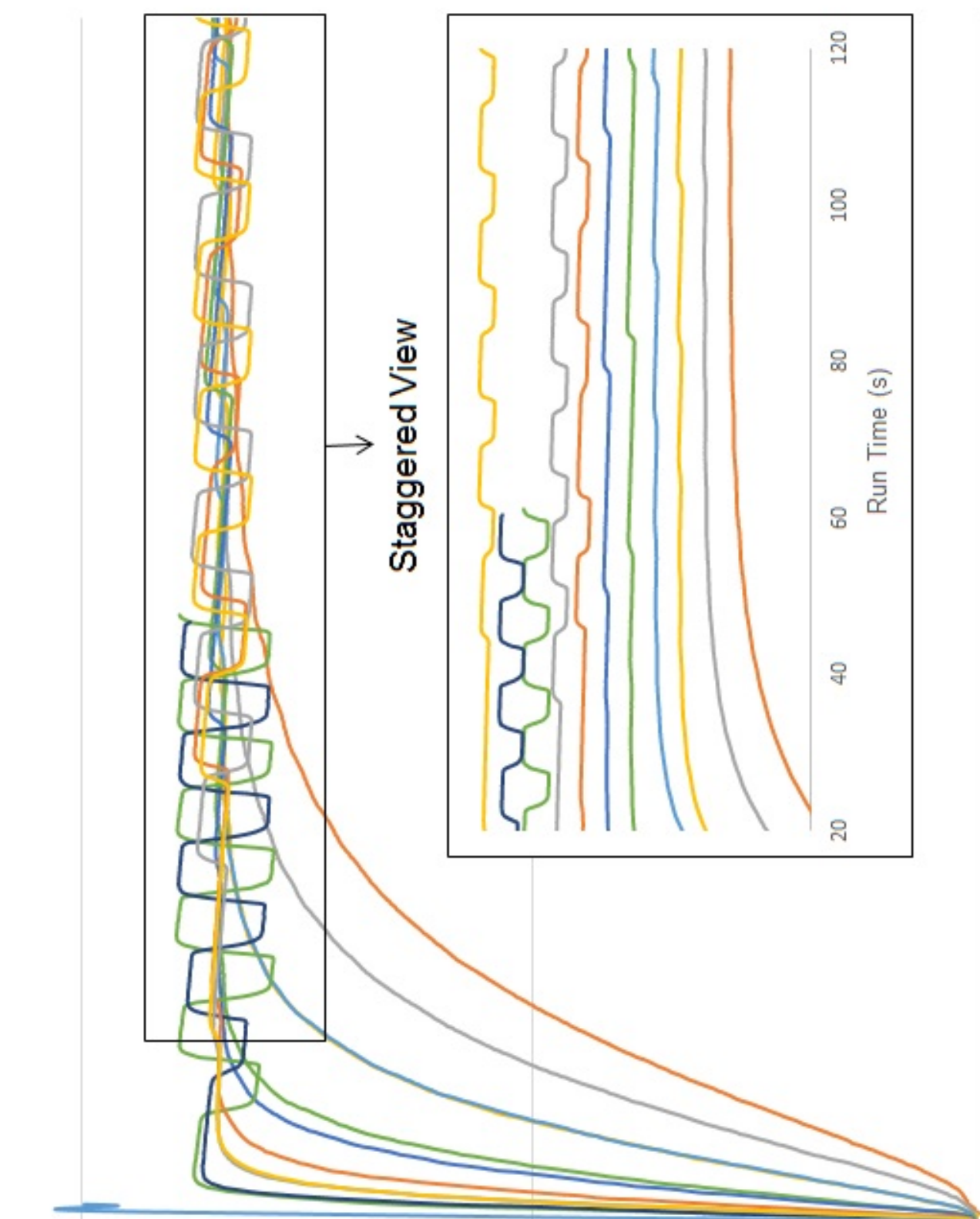

옥

족

$\frac{\text { f }}{\frac{5}{5}}$

움

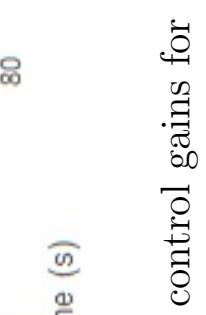

8

点

需

$+$

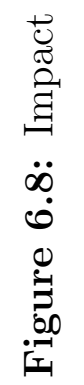

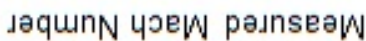


As can be seen, larger gain values provide much better settling times but tend to have poor steady state performance. For $K_{P}=20, K_{I}=20$, and $K_{D}=0$ the Mach number greatly overshoots (Largest Mach $=1.02$ or 0.17 overshoot) and the test was aborted in emergency to protect the transducers.

\subsection{2 "Small" Gain Values}

The seemingly very good steady state performance for $K_{P}=1, K_{I}=1$, and $K_{D}=1$ makes an investigation into "small" PID gains of interest. The resulting Mach performance for "small" gains can be seen in figure 6.9. 


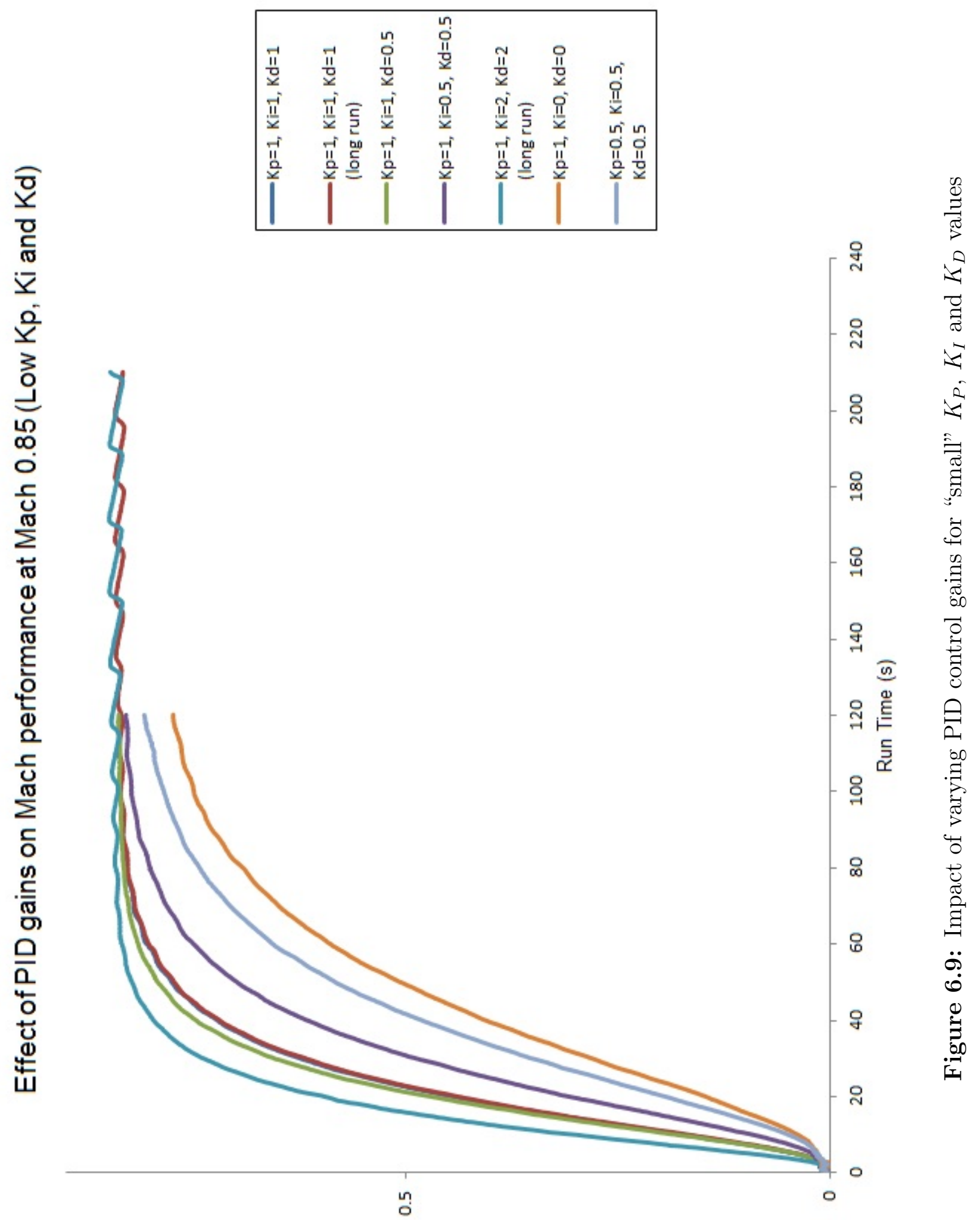

JaqunN YoeW pasnseaW 
Unfortunately the small $K_{p}$ values lead to incredibly long settling times and unacceptable transient performance. For this reason the steady state performance is difficult to observe without lengthy runs.

\subsection{Non-Linear Proportional Gain}

In an attempt to get the best of both worlds, that is a fast response time from a large $K_{p}$ and a good steady state response from a small $K_{p}$, a non-linear proportional gain is implemented (referred to as gain scheduling in control theory). When the measured Mach number is below $95 \%$ of the target Mach number a transient gain is used. Once the Mach number is above $95 \%$ of the target, a steady state gain is used. It should be noted that this non-linear gain is one-sided, that is an overshoot will not cause the transient gain to be used. The response for a variety of non-linear proportional gain values is shown in figure 6.10. The nomenclature used in the plots is $K_{P}=15 / 3$, where 15 would be the transient value and 3 the steady state value (not a $K_{P}$ of $15 / 3=5$ ). 


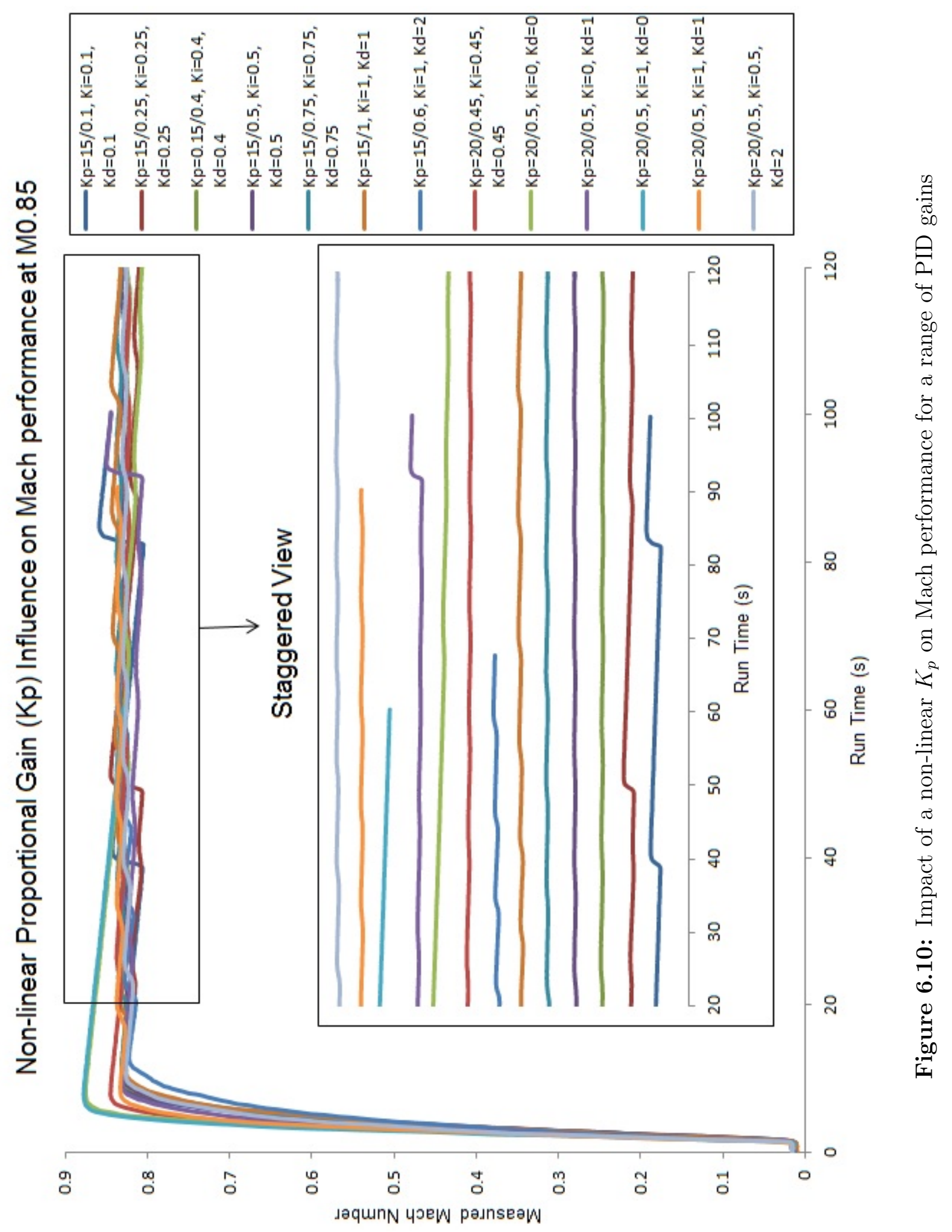


As can be seen, a transient $K_{P}$ value of 20 exhibits quite a good response time but is deemed to overshoot thus a transient gain of 15 is used. The steady state performance for low $K_{P}$ gains can be evaluated with this configuration given the decreased settling times. A few combinations seem to perform adequately. $K_{P}=15 / 0.4, K_{I}=0.4$, $K_{D}=0.4$ and $K_{P}=15 / 0.5, K_{I}=0.5, K_{D}=0.5$ and $K_{P}=15 / 0.75, K_{I}=0.75, K_{D}=0.75$ and $K_{P}=20 / 0.5, K_{I}=0.5, K_{D}=2$ seem to perform quite well thus steady state performance obtained from these combinations is examined further.

For brevity only the analysis for $K_{P}=15 / 0.5, K_{I}=0.5, K_{D}=0.5$ is shown as this combination exhibits the best performance. The other combinations are comparable but with greater oscillation amplitudes. The steady state response for these gains is shown in figure 6.11. The unsteadiness is estimated as the average peak to peak distance (the two red lines). The linear trendline from 20 to 120 seconds is also shown. The slope of this trend line is found to be a function of the steady state gains used. Gains too small cause the slope to be negative whereas gains too large cause it to be positive. The peak to peak variation is approximately Mach 0.004 and the average increases with a slope of 2e-5. As mentioned previously, this is the best performance obtained from any PID gain values thus these are kept for the remainder of this work.

Unfortunately, the ideal gains are found to be a function of both tank pressure and target Mach number. For given gains, the slope of the steady state trend line for Mach numbers higher than 0.85 is negative and becomes increasingly negative for larger Mach numbers. Ideally non linear steady state gains would be employed as a function of the Mach number, however, this is beyond the scope of this work. After many days of testing the ideal gain values were found to vary slightly based on the atmospheric pressure but a compensation for this is also beyond the scope of this work. 


\section{Steady State Mach Performance with Non-Linear Proportional Gain Kp $15 / 0.5, \mathrm{Ki}=0.5$ and $\mathrm{Kd}=0.5$ at Mach 0.85}

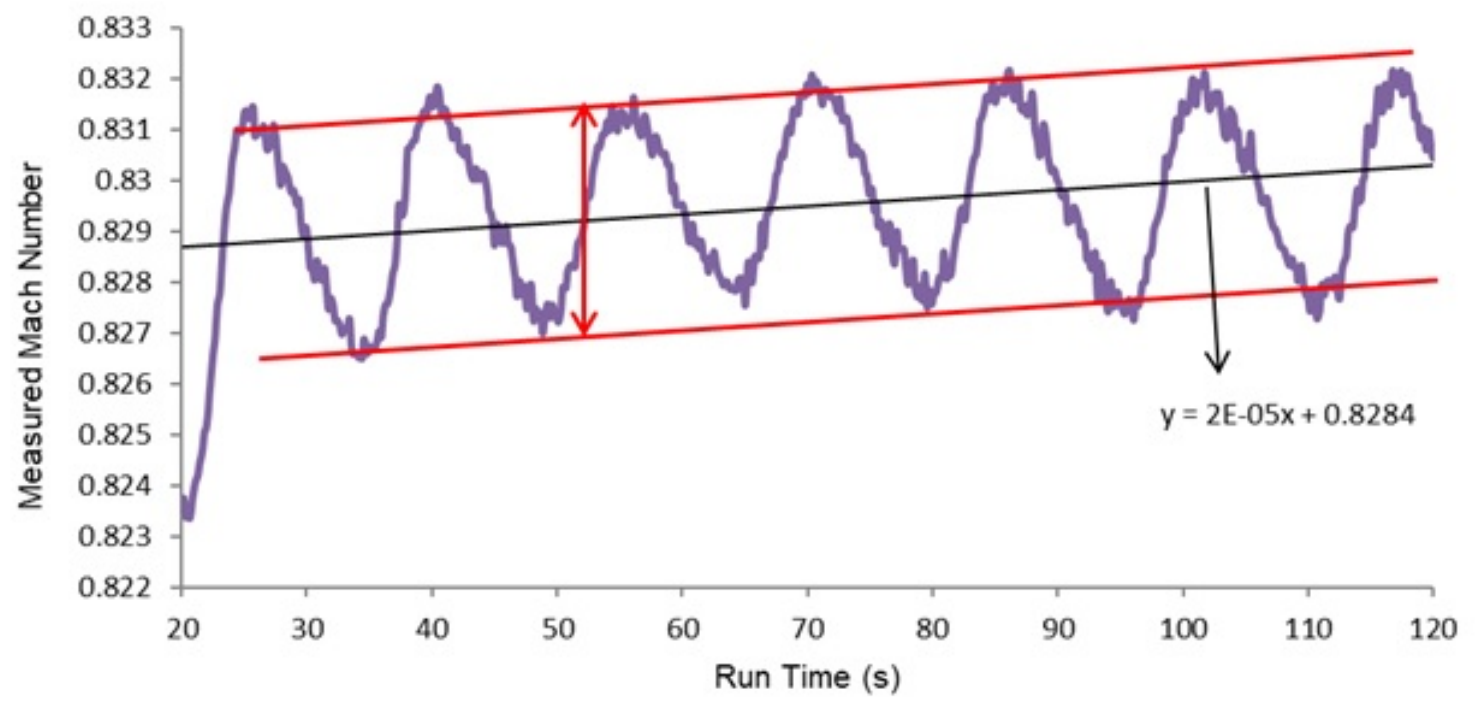

Figure 6.11: Steady state analysis of the Mach performance for a non-linear $K_{P}$ of $15 / 0.5, K_{I}$ of 0.5 and $K_{D}$ of 0.5

Figure 6.12 shows the steady state performance of the entire Mach range obtained with this non-linear gain combination of a $K_{P}$ of $15 / 0.5$, a $K_{I}$ of 0.5 and a $K_{D}$ of 0.5. The steady state is reached after 10-15 seconds, depending on the Mach number. The oscillations are deemed of an acceptable magnitude, however, it should be noted that there is a significant Mach undershoot, especially at high Mach numbers. 


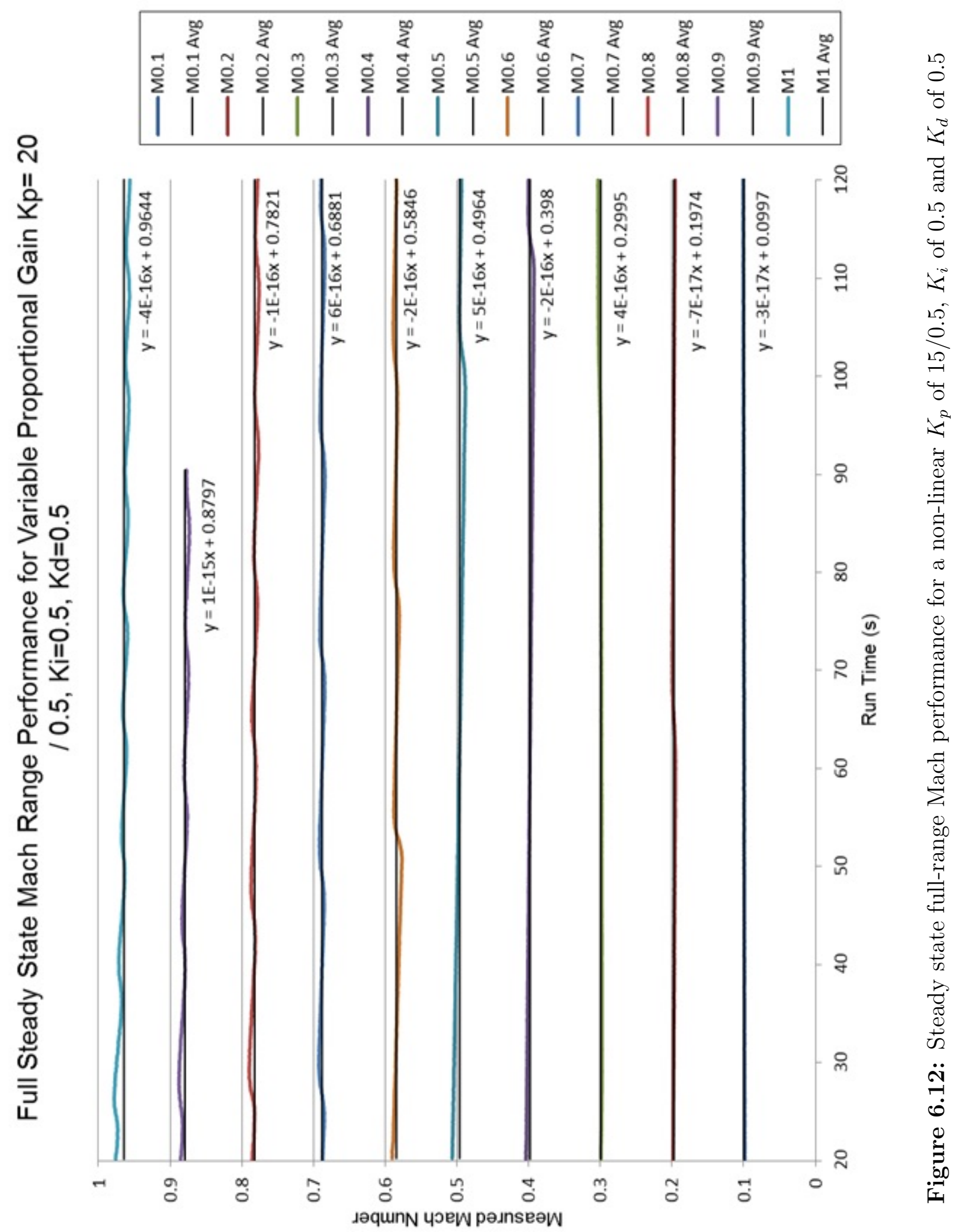




\subsection{Mach Undershoot}

An existing compensation is built into the code to correct the Mach number undershoot. This compensation requires the user to input an undershoot value (determined experimentally) based on the target Mach number. The code adds the undershoot to the target and uses the new value as the actual target Mach number 5 . Given the fast pace at which testing occurs with scale model (one test run approximately every 3 minutes as opposed to one test every half hour for the tests of Giardino), a manually input undershoot correction is deemed inappropriate. For this reason, a polynomial best fit Mach undershoot correction is added to the code.

Figure 6.13 is a plot of the average steady state Mach undershoot values for each Mach number ${ }^{6}$. A second order polynomial is fit to these data points, providing an approximate formula for predicting the Mach undershoot at different Mach numbers.

The Mach undershoot compensation is modified such that an undershoot value is estimated in real time based on the measured Mach number. This undershoot value is used to adjust the target Mach number providing a non-undershot Mach number for all Mach numbers. The steady state Mach performance obtained using this compensation is shown in figure 6.14. This performance is much better than the baseline (figure 6.1) and the control parameters giving this performance are used for the remainder of this work. It is important to note that the Mach undershoot is a function of the control gains and tank pressure, thus if low-pressure tests are done or new control gains are used the resulting Mach performance will likely be poor.

An estimate of the average velocity and oscillation amplitude can be made from

\footnotetext{
${ }^{5}$ Essentially the same as entering Mach 0.92 instead of Mach 0.9 if it is known that the offset at Mach 0.9 is 0.02

${ }^{6}$ Note that Mach 0.6 appears to be an outlier, however, this trend remains true for multiple tests. The reason for this is unknown.
} 


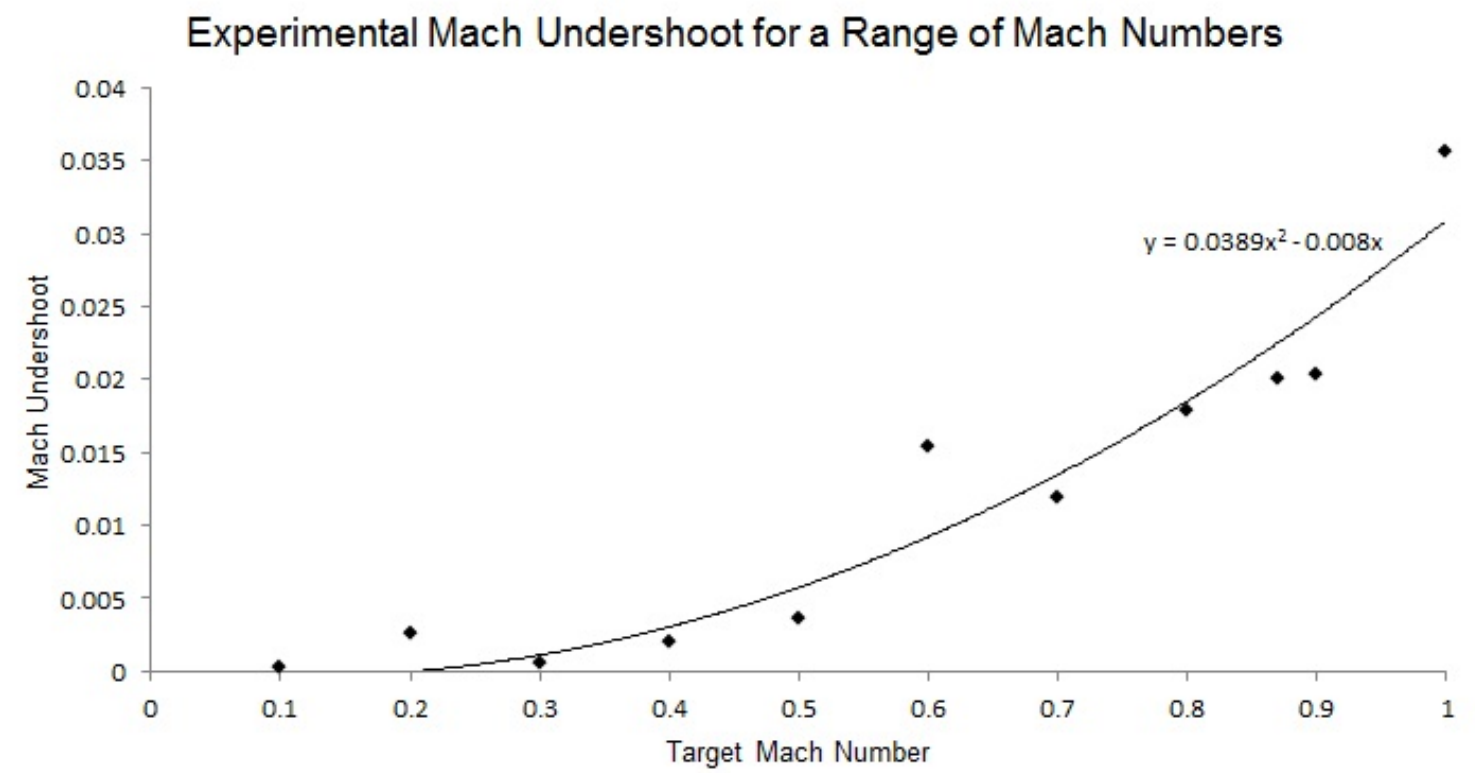

Figure 6.13: Values of Mach undershoot with the best fit polynomial used to predict and correct for the undershoot

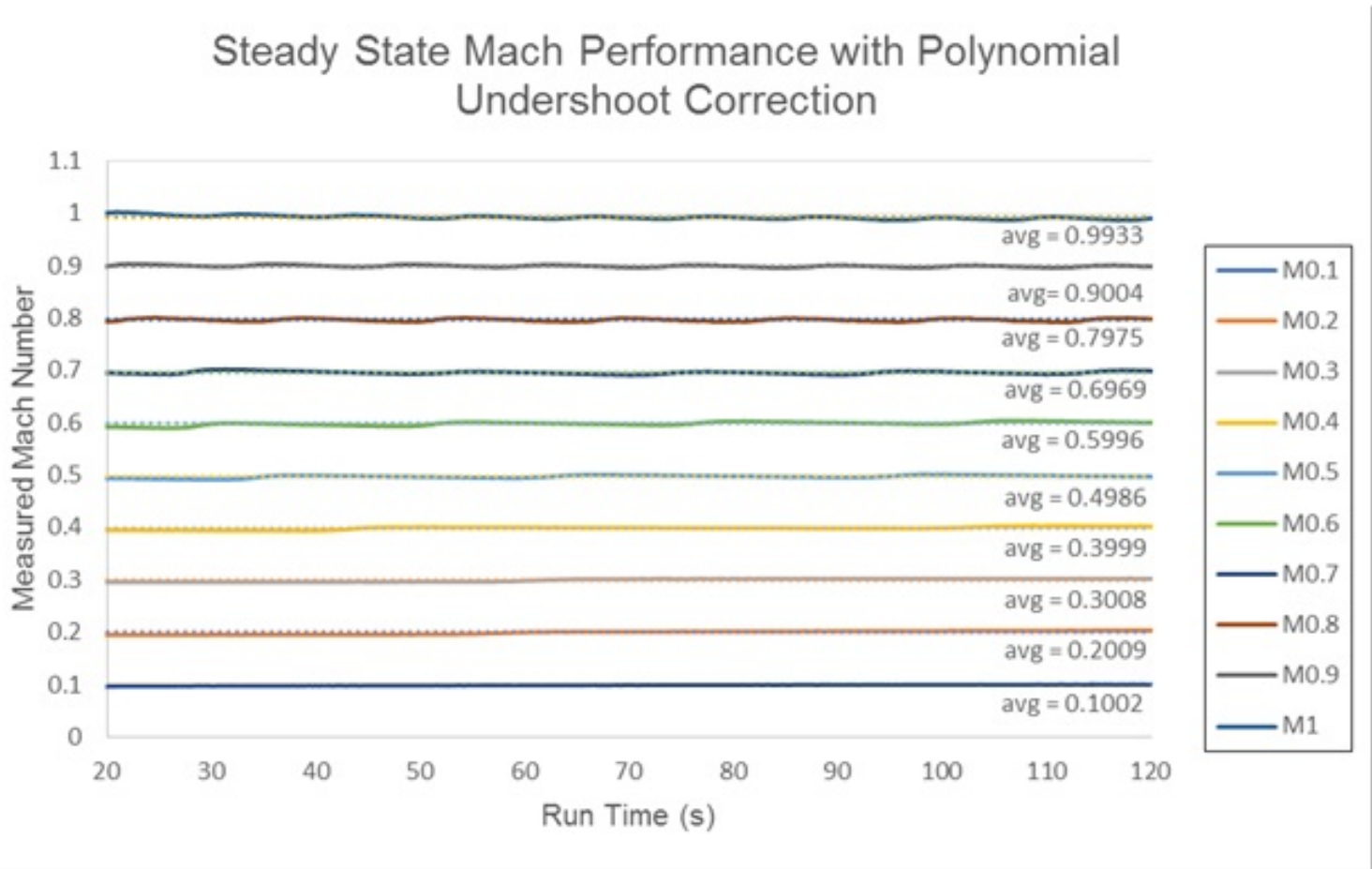

Figure 6.14: Steady state Mach performance with non-linear PID gains and polynomial Mach undershoot correction 
these runs. Table 6.1 provides a summary of the average and standard deviation of the measured steady state Mach number. Neglecting the transducer uncertainty, it can be estimated that $95 \%$ of measurements will fall within \pm 2 standard deviations of the mean value. As mentioned in the measurement uncertainty section, the uncertainty due to the steady state oscillations is an order of magnitude less than the transducer uncertainty. The primary nozzle calibrations are not applied at this point, nonetheless, the performance is typical of the fully calibrated setup. The values presented are found to vary day to day thus they should only be viewed as an estimate 7 .

Table 6.1: Average Measured Mach Number, Standard Deviation and Uncertainty Range for a Variety of Target Mach Numbers

\begin{tabular}{cccc}
$\begin{array}{c}\text { Target Mach } \\
\text { Number }\end{array}$ & $\begin{array}{c}\text { Average Measured } \\
\text { Mach Number }\end{array}$ & $\begin{array}{c}\text { Standard } \\
\text { Deviation }\end{array}$ & $\begin{array}{c}95 \% \text { of Observations } \\
\text { Range }\end{array}$ \\
\hline 0.1 & 0.100 & 0.001 & $0.098-0.102$ \\
0.2 & 0.200 & 0.003 & $0.194-0.206$ \\
0.3 & 0.300 & 0.003 & $0.294-0.306$ \\
0.4 & 0.399 & 0.003 & $0.393-0.405$ \\
0.5 & 0.499 & 0.003 & $0.493-0.505$ \\
0.6 & 0.599 & 0.003 & $0.593-0.605$ \\
0.7 & 0.697 & 0.003 & $0.691-0.703$ \\
0.8 & 0.798 & 0.002 & $0.794-0.802$ \\
0.9 & 0.900 & 0.002 & $0.896-0.904$ \\
1 & 0.993 & 0.003 & $0.987-0.999$
\end{tabular}

\footnotetext{
${ }^{7}$ For example, on days with low atmospheric pressure the Mach undershoot compensator does not fully compensate. This error is minor and has not been quantified
} 


\subsection{Primary Nozzle Calibration}

With the gains and Mach undershoot resolved, the primary nozzle can be calibrated. Up to this point, the pressure ratio from the Pitot-static probe is used for control with the primary nozzle pressure ratio being monitored and recorded but not used for control. In order to obtain quality acoustic measurements it is important to operate the primary nozzle without the Pitot-Static probe in the jet potential core.

\subsubsection{Total Pressure Calibration}

The primary nozzle total pressure is found to be consistently higher than the Pitotstatic total pressure, which is expected. Many metrics comparing the two (including the gage pressure ratio, absolute pressure ratio, gage pressure difference and absolute pressure difference to name a few) are evaluated in an attempt to obtain the best total pressure calibration. The best correlation is found by comparing the gage pressures at a given time. Figure 6.15 shows this relationship for over 7000 data points. The best fit curve equation is used to correct the total pressure. Much like the Mach undershoot, the total pressure is corrected in real time based on the total pressure measurement. A non-linear polynomial is used to improve the curve fit correlation.

\subsubsection{Static Pressure Calibration}

Unlike the total pressure, none of the static pressure metrics show any clear correlation. This is likely due to the Pitot-static probe being too close to the nozzle outlet, however, it cannot but moved any further back due to model constraints. In order to avoid integrating any errors relating to the absolute pressure measurement, the gage pressure ratio is used to calibrate the primary nozzle static pressure readings. The 


\section{Total Pressure Ratio for Primary Nozzle Total Pressure Calibration}

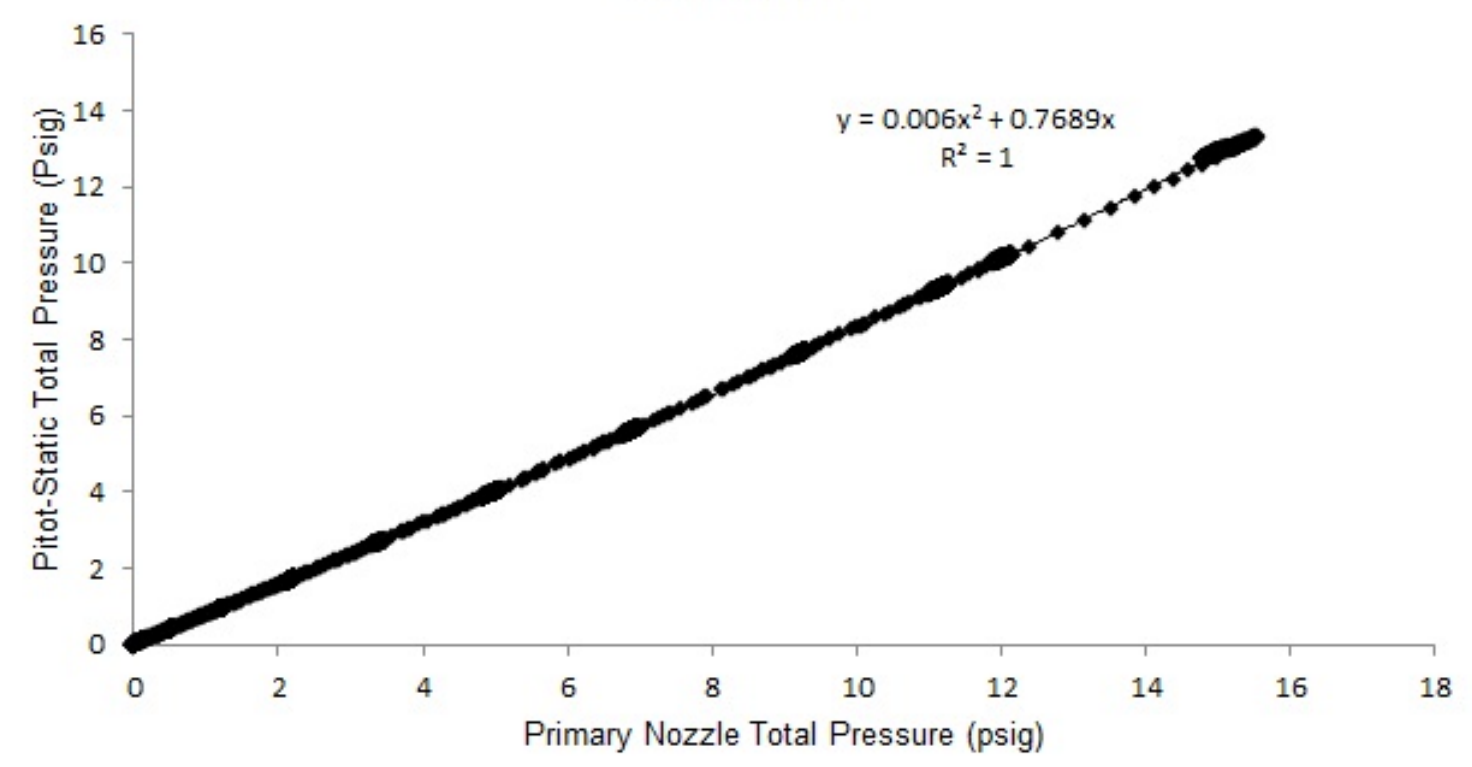

Figure 6.15: Relationship between the primary nozzle total pressure and the Pitotstatic total pressure

steady state average static pressure ratio is determined for each Mach number and is plotted against the measured Mach number, with the resulting plot shown in figure 6.16. Again, a polynomial is fit to these points and used to correct the pressure in real time. The polynomial fit is approximate, however, the gage primary nozzle static pressure readings are small. When added to the atmospheric pressure the error due to the calibration is deemed negligible.

\subsubsection{Mach Verification}

As a final verification that everything is working as anticipated, the primary nozzle velocity is controlled using the primary nozzle pressure ratio with all the aforementioned corrections and calibrations applied. The Pitot-static probe is placed in the potential core in order to verify the primary nozzle velocity. The amount of time 


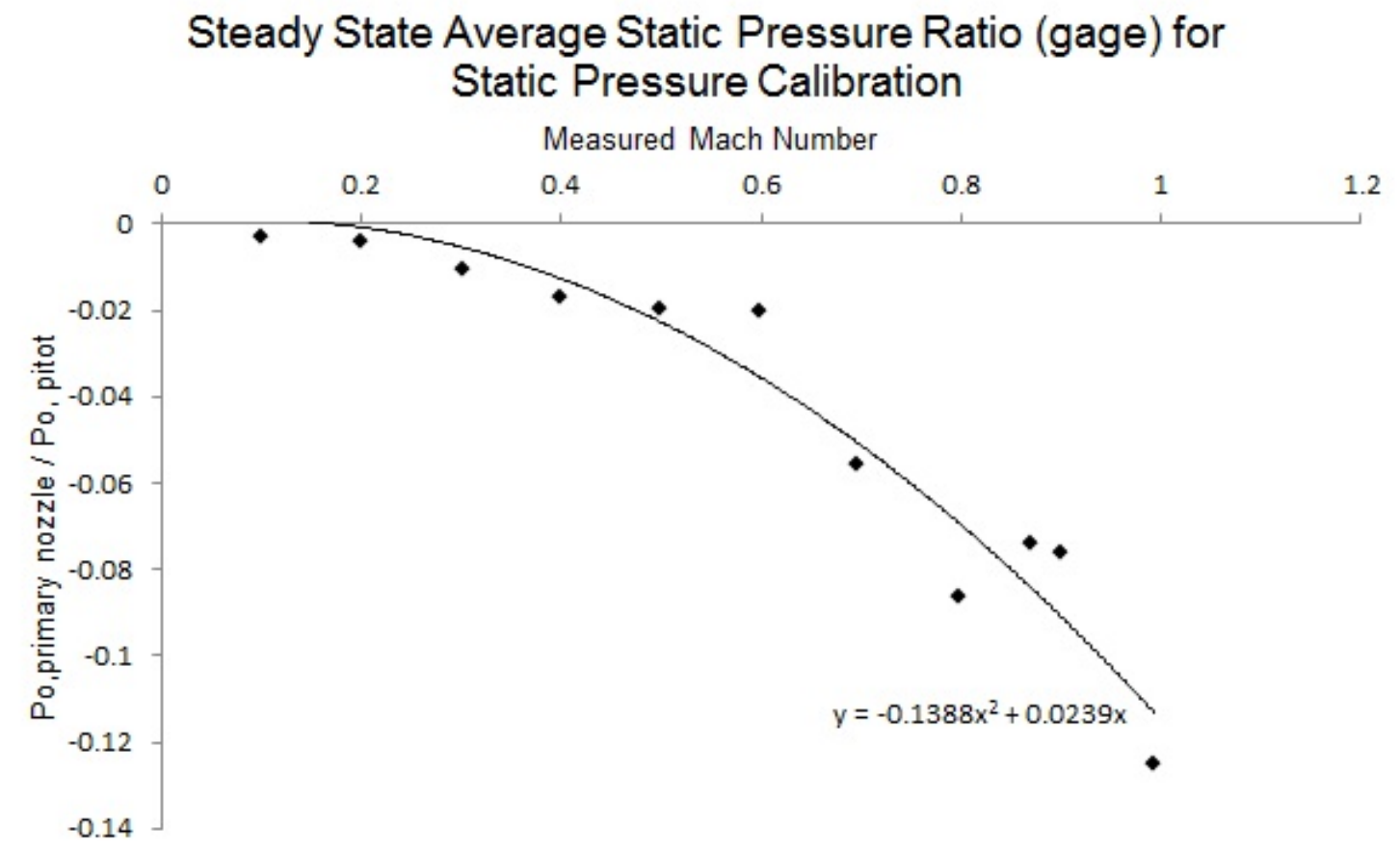

Figure 6.16: Static pressure ratio between the primary nozzle and Pitot-static probe for a range of Mach numbers

where the Mach number is zero is found to increase when using the primary nozzle for control but this is simply solved by increasing the slack voltage to 3.9 volts. A comparison of the Mach number measured by the Pitot-static probe and measured by the primary nozzle pressure ratio at Mach 0.8 is shown in figure 6.17 qualitatively verifying that the Mach number control provides the desired Mach number within an acceptable limit.

\subsection{Summary}

To summarize, a transient proportional gain $K_{P}$ of 15 , a steady state proportional gain $K_{P}$ of 0.5 , an integral gain $K_{I}$ of 0.5 , a derivative gain $K_{D}$ of 0.5 , a valve slack of 3.9 volts, no deadband compensation, a polynomial Mach undershoot correction 


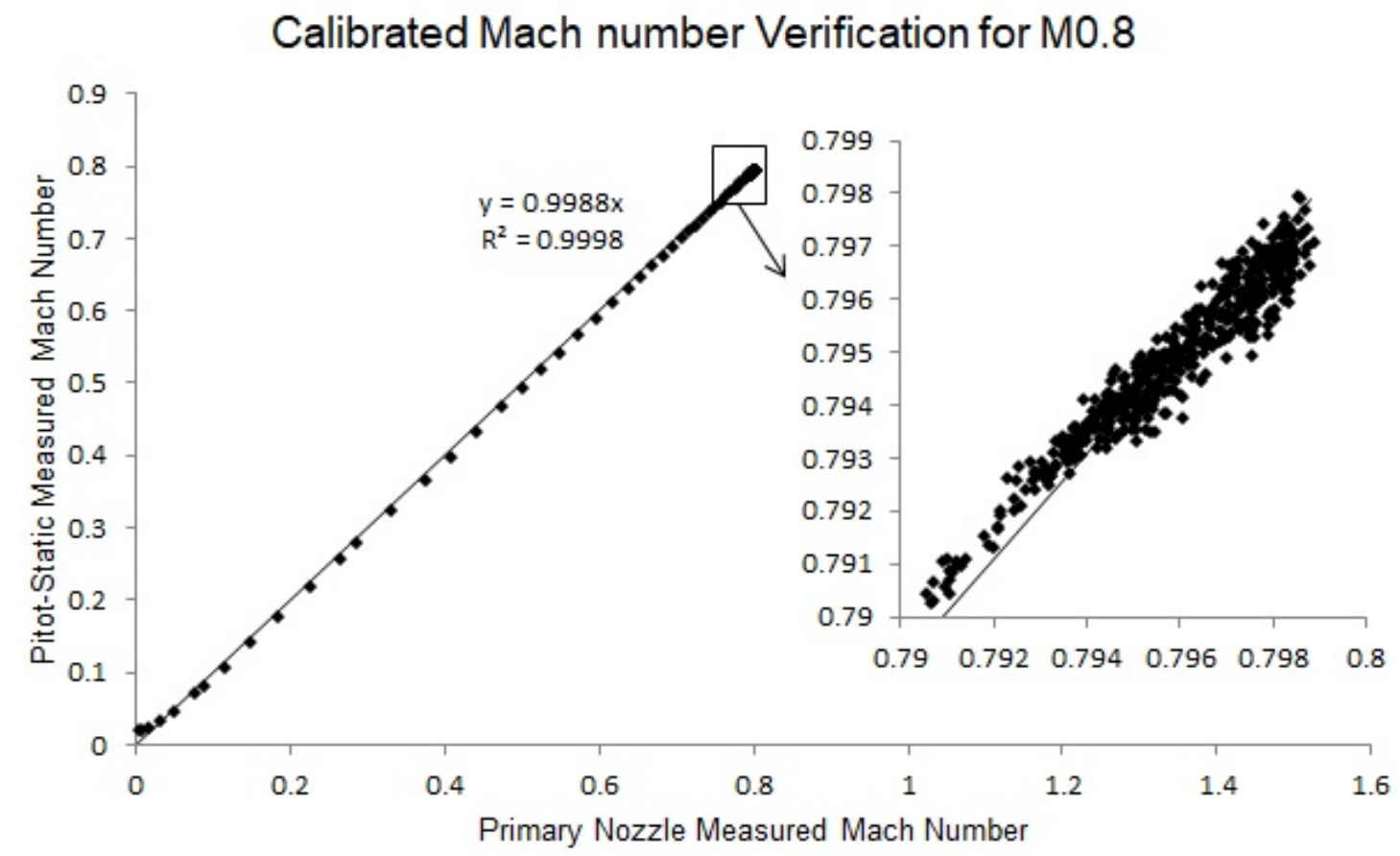

Figure 6.17: A comparison of the primary nozzle Mach number measurement and Pitot-static Mach number measurement, showing a high correlation

of $y=0.0389 x^{2}-0.008 x$ (where $y$ is the undershoot correction value and $x$ is the measured Mach number), a polynomial primary nozzle total pressure calibration of $y=0.0006 x^{2}-0.7689 x$ (where $y$ is the corrected total pressure and $x$ is the primary nozzle total pressure) and a polynomial primary nozzle static pressure calibration of $y=-0.1388 x^{2}-0.0239 x$ (where $y$ is the gage pressure ratio correction factor and $x$ is the measured Mach number) are used throughout this work. 


\section{Chapter 7}

\section{Results I: Facility and Model Characterization}

With the nozzle velocity being reliably controlled, extensive characterization of both the scale model and the HSWT facility is undertaken. This chapter presents the characterization of acoustic sources in the HSWT, the acoustics of the baseline scale model configuration (that is the configuration without any modifications) and the aerodynamic performance of the scale model in the baseline configuration. Details on the acoustic data post processing techniques are also presented in this chapter.

The determination of mechanical resonance frequencies of the components/assemblies of the scale model was considered but not undertaken due to time constraints. Third party tests of natural acoustic modes of scale model ejector components (such as the throat and secondary flow chamber) were found to be within a very close margin of the theoretical modes therefore an analysis of this type is not performed. 


\subsection{Baseline Microphone Performance}

\subsubsection{Microphone Data Analysis Procedure}

For all acoustic results, the time and the fluctuating pressure (in Pascals) for each microphone is recorded. The data is used to obtain OASPL readings as well as SPL plots.

The data is first cut to 30 seconds (when required) and passed through a time domain filter in order to remove low and high frequency contamination caused by the microphone low frequency roll off and high frequency aliasing. The bandpass filter used is a Butterworth filter with the first and second stopband frequencies of 25 and

$\frac{f_{s}}{2} \mathrm{~Hz}$ respectively, where $f_{s}$ is the sampling frequency. The first and second passband frequencies are 50 and $\frac{f_{s}}{2}-25 \mathrm{~Hz}$ respectively. The attenuation is $60 \mathrm{~dB}$ for both the first and second stopbands. The passband ripple is kept as the $1 \mathrm{~dB}$ default and the band match used is 'stopband'. The overall sound pressure level, or OASPL, is computed using the root mean square (RMS) of the filtered pressure using equation 7.1 .

$$
O A S P L=10 \log _{10} \frac{P_{R M S}^{2}}{P_{r e f}^{2}}
$$

where $P_{\text {ref }}$ is the standard reference pressure in air of 20 micro Pascals.

The filtered pressure data is converted to the frequency domain using the MATLAB Pwelch function with a window of 800 (good compromise between smoothness and frequency resolution), default overlap, default frequency vector, input sampling frequency and 'power' option. The resulting squared pressure for each frequency band is converted to a Sound Pressure Level using equation 7.1 and is plotted against frequency. The Pwelch function only returns frequencies up to half of the sampling 
frequency thus the Nyquist Sampling Criterion is automatically satisfied.

\subsubsection{Microphone Calibration Verification}

A Svantek model SV 34 (114 dB tone at $1000 \mathrm{~Hz})$ sound pressure level meter calibrator owned by Carleton Environmental Health and Safety is used to verify the calibration of the microphones. This calibrator is designed for $1 / 2$ inch $(12.7 \mathrm{~mm})$ microphones thus a small gap exists between the calibrator and the $1 / 4$ inch $(6.35$ $\mathrm{mm}$ ) microphones. Acoustic foam is used to hold the microphones (with gridcaps) in place in the oversized hole. Figure 7.1 shows the SPL plot for microphone 1 as measured at a sampling rate of $10 \mathrm{kHz}$. The resulting output is as expected with an OASPL discrepancy of only $1 \mathrm{~dB}$, potentially due to the foam or gridcap. The other microphones performed very similarly thus the default calibrations are used for the remainder of the work based on these results. The calibration plots for the remaining microphones can be found in Appendix A.

\subsubsection{Microphone Noise Floor}

An analysis of the microphone noise floor, that is the minimum noise recordable by the microphone partly due to self noise, is done with all 5 microphones in situ in the scale model in the Anechoic chamber. Again, all 5 microphones show nearly identical noise floors thus only that of microphone 1 is presented in figure 7.2 . Any noise with levels below this noise floor can not be captured by the microphones. This test was performed with the heater on, as would be the case in regular testing. 


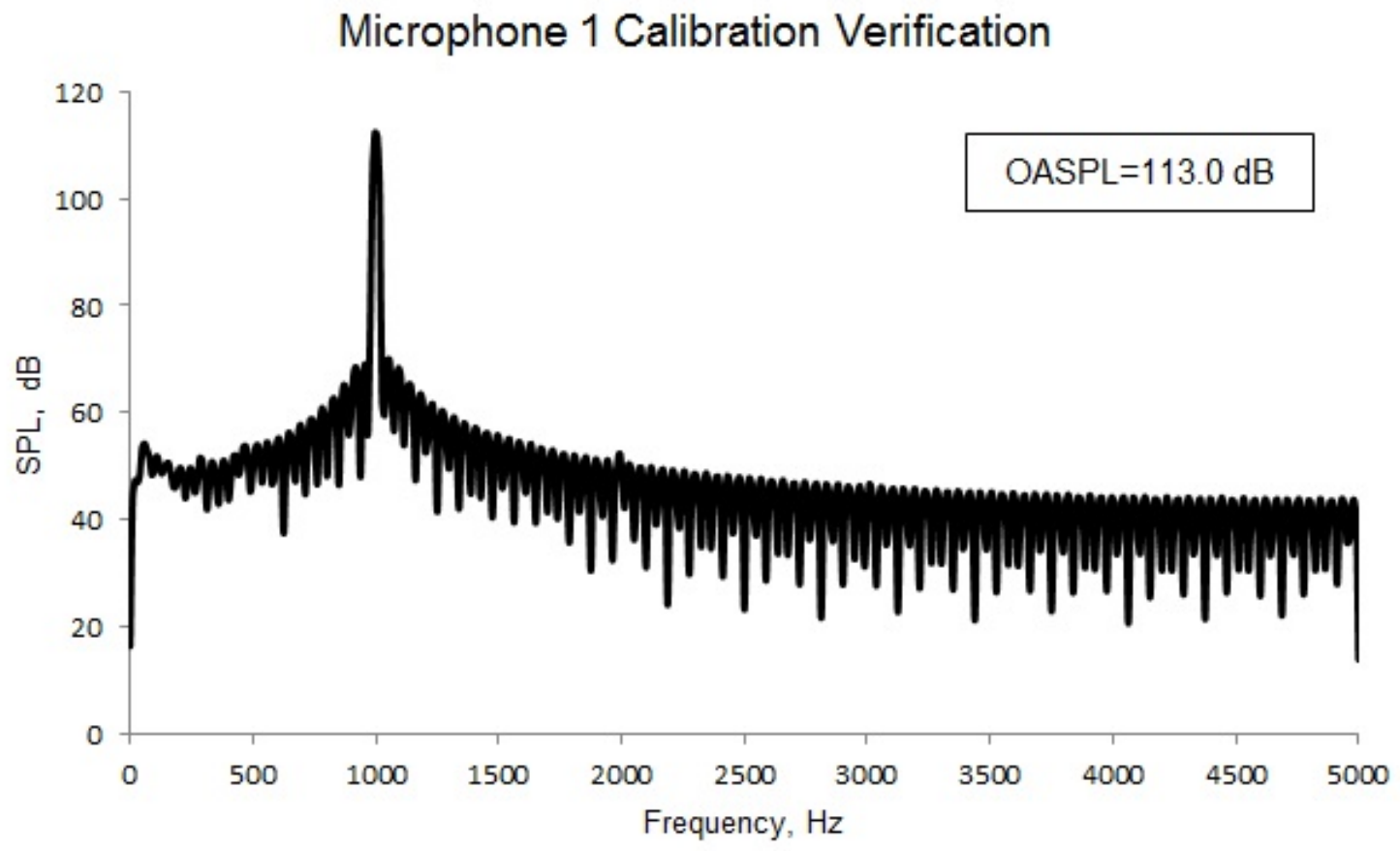

Figure 7.1: The calibration result obtained for microphone 1

Microphone 1 Noise Floor

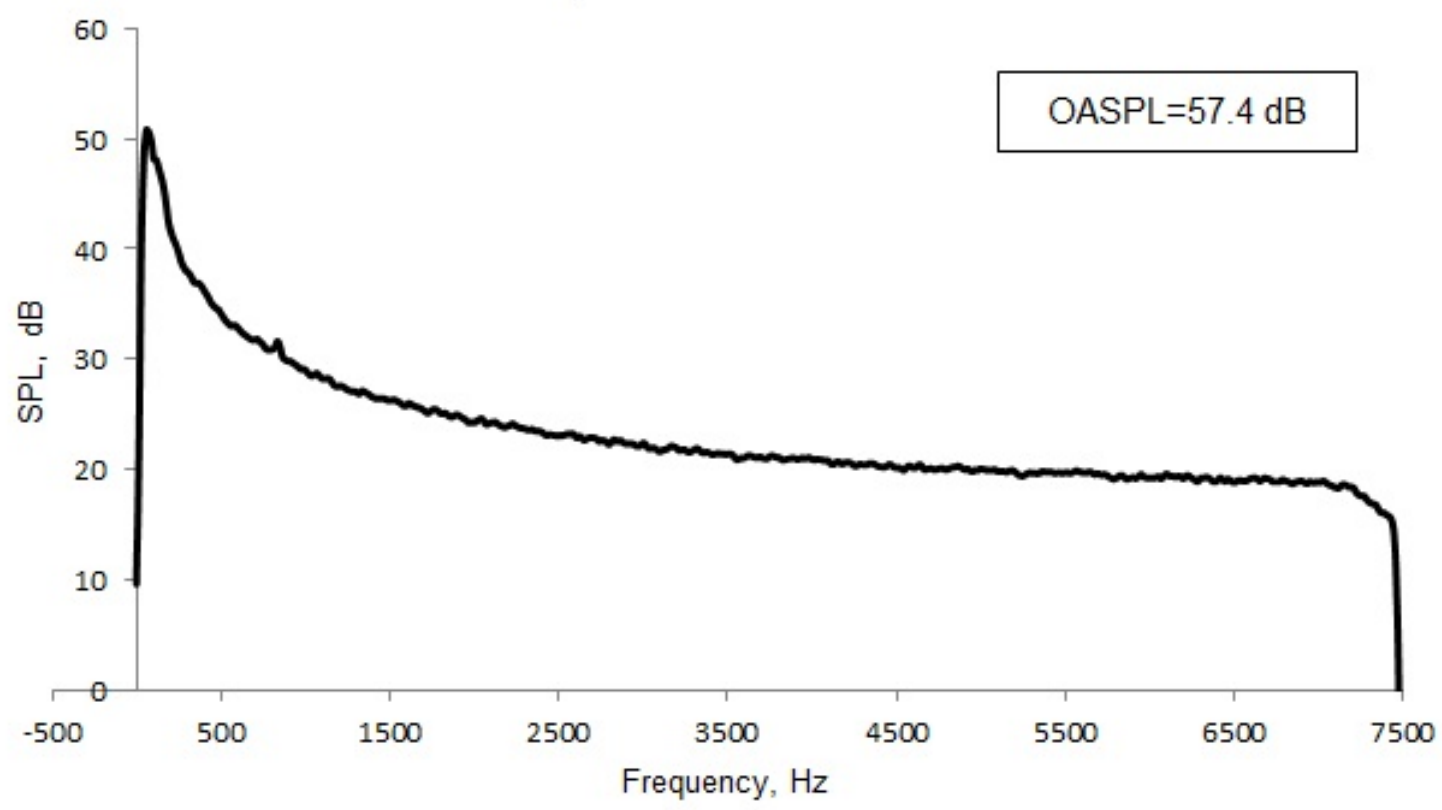

Figure 7.2: The noise floor obtained for microphone 1 


\subsection{Background Acoustic Characterization}

Other than the preliminary measurements of Giardino (in-chamber), no acoustic measurements have been made in the HSWT. This means that no effort has ever been put into quantifying the various noise sources in the HSWT which could affect in-chamber microphone readings. The noise sources of interest include the noise generated by the heater, noise generated by the air dryer, noise generated at the valve and a resonance in the flexible air hose. These components can all be seen in figure 4.9.

\subsubsection{Heater Noise}

Although not terribly loud, the heater operates intermittently and it is acoustically characterized for this work. A microphone is mounted to a crane with acoustic foam and placed three feet from and 45 degrees below the center of the heater. The acoustic signature of the heater, recorded at $40 \mathrm{kHz}$, is shown in figure 7.3 . As can be seen, the heater is realtively quiet with no distinct frequency spikes.

\subsubsection{Air Dryer Noise}

The air dryer used to remove moisture from the air before it enters the storage tanks emits quite a loud tone which is more pronounced at lower tank pressures. Upon recording the tone it was observed that the nature of the tone changes with tank pressure. The measurements are taken as the tanks are being filled from empty. A microphone is placed on a support post 42 inches (1.07 meters) off the ground and 41 inches (1.04 meters) from - and perpendicular to - the dryer control panel. Data is acquired at a rate of $40 \mathrm{kHz}$. The recorded dryer noise is not isolated but rather it includes the noise from air expansion into the tanks and from air being passed 


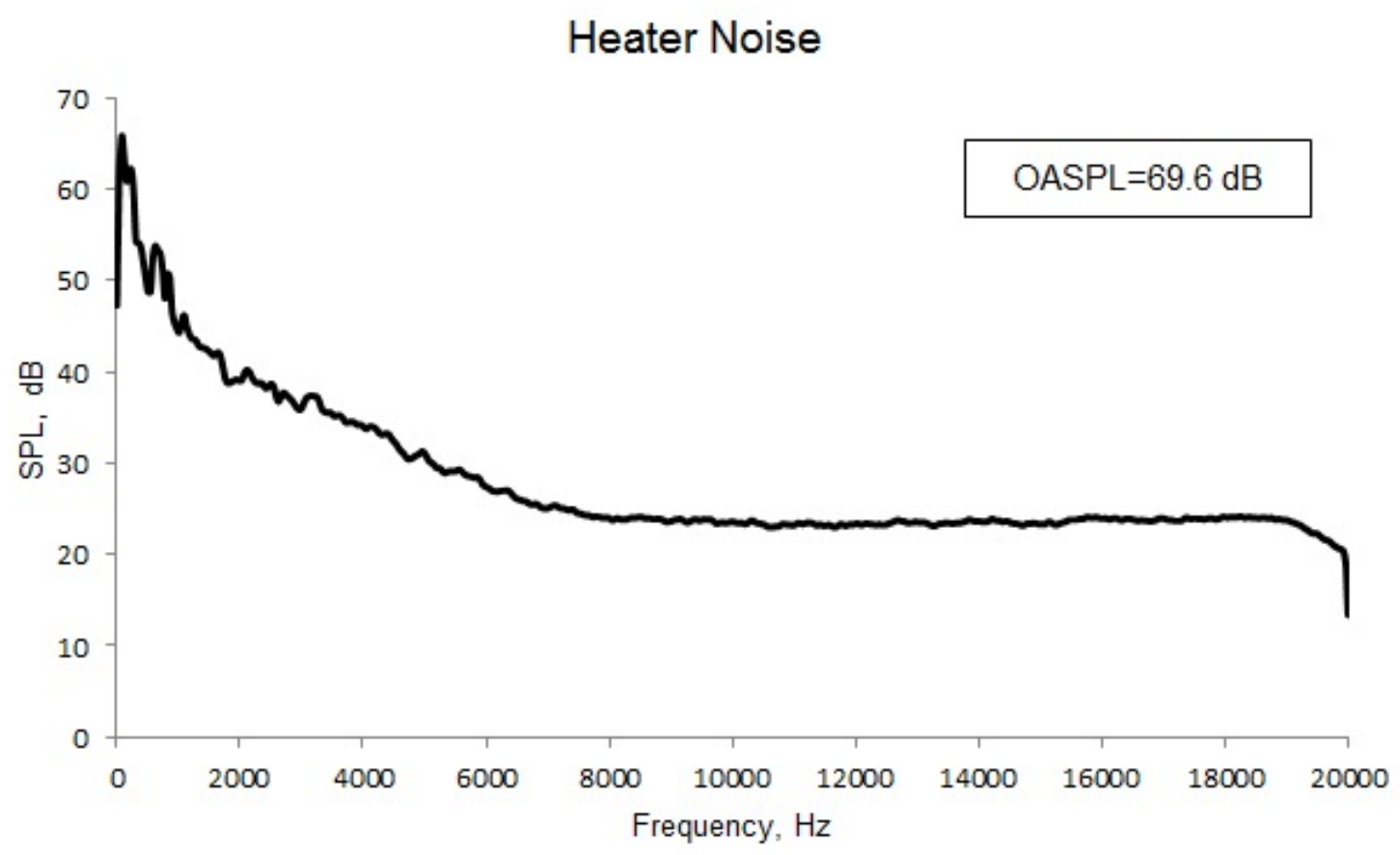

Figure 7.3: Acoustic signature of the HSWT heater

through the dryer. Nonetheless, the dryer tone is clear on the acoustic spectrums. The dryer sometimes switches from one tower to the other in about half a second, a process which emits noise. This noise is captured and analyzed and found to have a negligible impact on a 30 second data capture.

Figure 7.4 shows the acoustics of the dryer for a tank pressure of $20 \mathrm{psi}(138 \mathrm{kPa})$, $30 \mathrm{psi}(207 \mathrm{kPa})$ and $35 \mathrm{psi}(241 \mathrm{kPa})$. At $20 \mathrm{psi}$ no tones are observed. At $30 \mathrm{psi}$ the tone of interest can be observed. At 35 psi this tone is found to change, with new frequencies observed. These tones are of fairly large magnitude and may have an impact on the scale model results. 


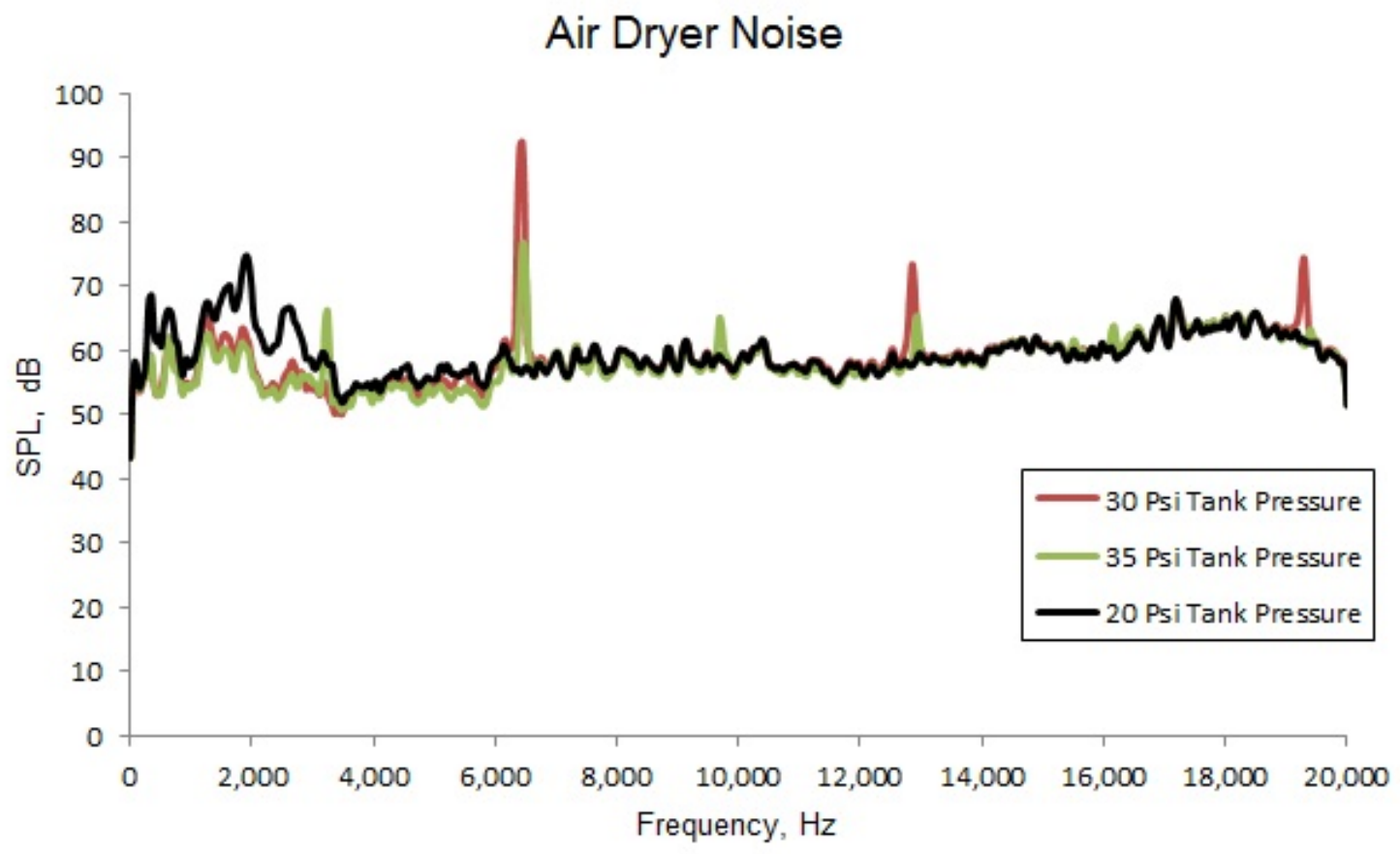

Figure 7.4: Acoustic signature of the HSWT dryer at 20, 30 and 35 psi tank pressure

\subsubsection{Silencer Transmission Noise}

Given that the dryer noise is recorded a short distance from the dryer, it is unknown how it will propagate to the chamber interior. In order to quantify the impact of the dryer noise inside the chamber, a microphone is centered immediately downstream of a silencer outlet (inside the chamber). Upon the dryer emitting a tone at $30 \mathrm{psi}$, the resulting noise in the chamber is captured at a rate of $15 \mathrm{kHz}$ and is shown in figure 7.5. As can be seen, the noise from the tone infiltrating the chamber is minimal, barely more than the microphone noise floor and only observed at the frequency of the tone. Based on this result, the silencers appear to do a good job and the dryer is not expected to affect scale model results. 


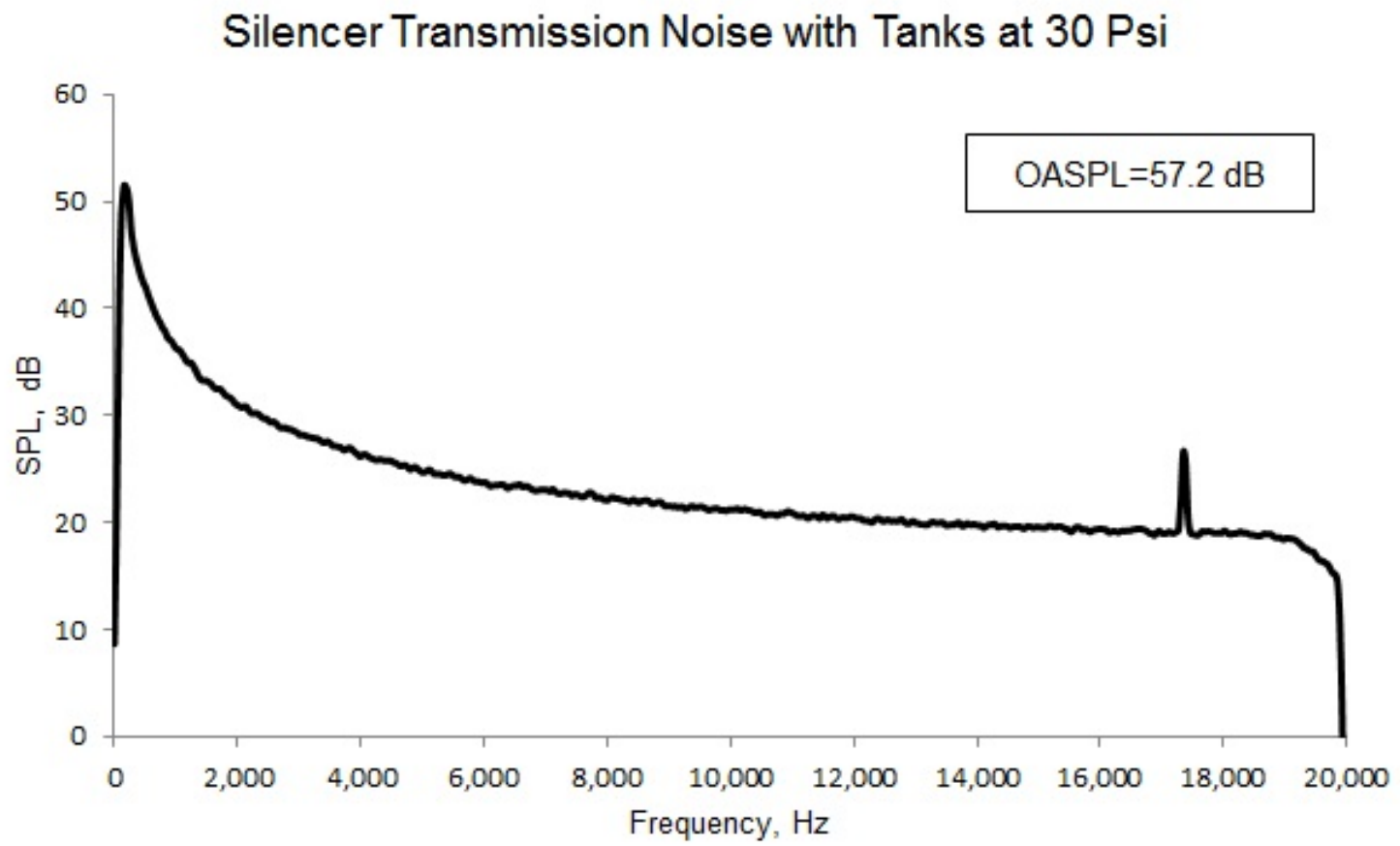

Figure 7.5: Acoustic signature downstream of the silencer inside the chamber with the dryer emitting a tone

\subsubsection{Noise at the Valve}

A significant source of noise is the noise created at the valve and T-junction. This noise is measured in line with the T-junction inlet at a distance of 3 feet (0.9 meters) and at the height of the junction at a sampling frequency of $40 \mathrm{kHz}$. The noise is captured for Mach numbers 0.1 - 1, in intervals of 0.1 , as well as at the design Mach number. For brevity only the noise signature at Mach 0.9 is shown in figure 7.6 with the full Mach range provided in Appendix C. Although referred to as "valve noise" it is difficult to isolate the true valve breakout noise and this component includes all noise sources occurring near the valve. This includes the noise generated as the air jet leaving the valve impinges on the wall of the T-junction. 


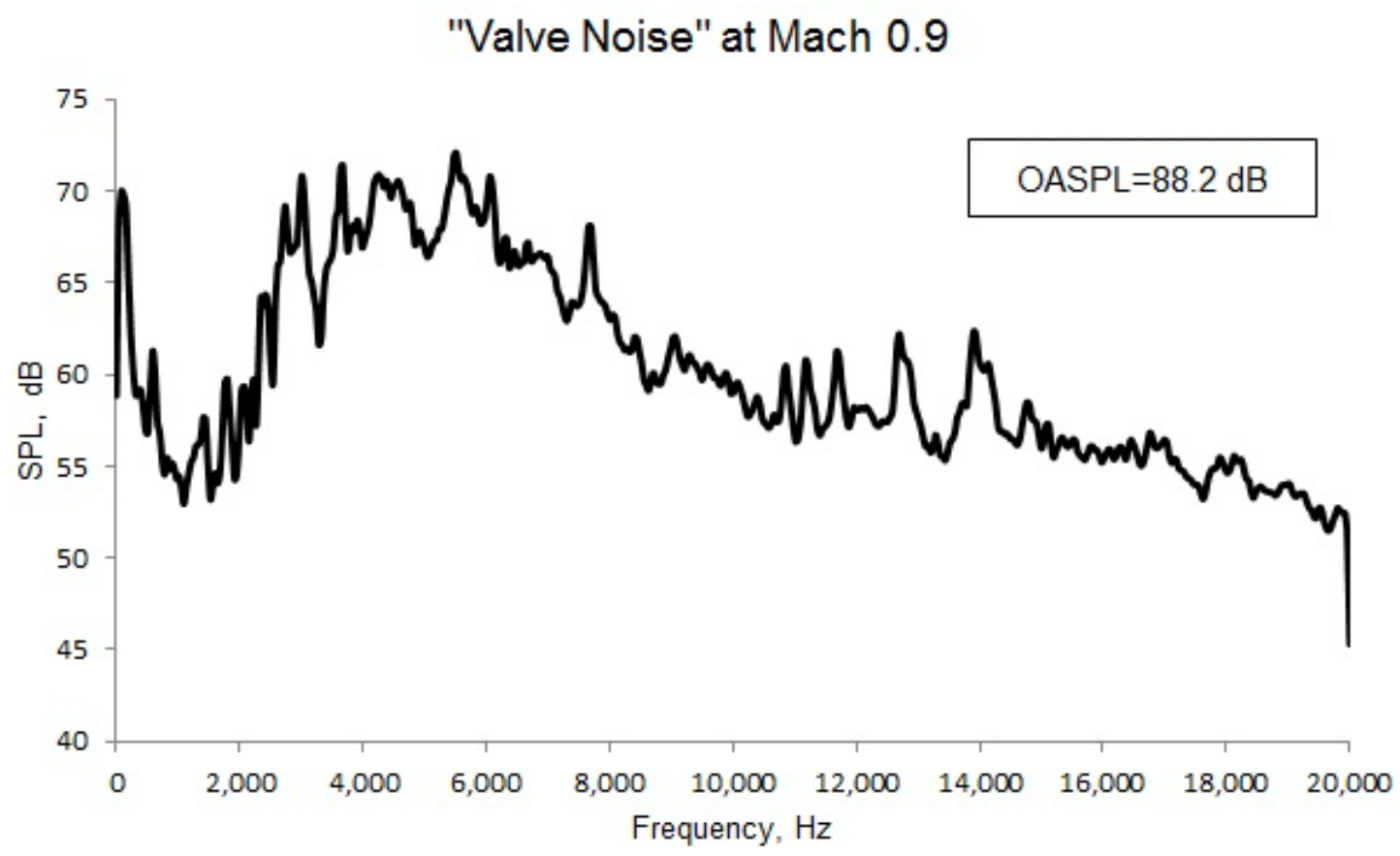

Figure 7.6: Acoustic signature of the "valve noise" at Mach 0.9

\subsubsection{Flexible Air Hose Resonance}

Upon assembling and disassembling the scale model, a strong resonance can be heard coming from the flexible air hose. The same noise can be heard in the control room during testing when the tank pressure is quite low (20 psi range), likely caused by an upstream disturbance exciting the natural modes of the hose. The resonance of the hose is measured in the anechoic chamber with a microphone approximately 3 inches (76 mm) from and perpendicular to the outlet of the hose at an acquisition frequency of $15 \mathrm{kHz}$. In order to excite the resonance, the outlet of the disconnected hose is hit with an open palm repeatedly for 30 seconds, with the opposite end connected to the T-junction with the control valve closed. The resulting acoustic spectrum is shown in figure 7.7 with the peak being rather wide, centered at $88 \mathrm{~Hz}$. The amplitudes in figure 7.7 bear little significance (the sound pressure level decreases with measurement distance and the excitation magnitudes are unknown in regular operation) to model 
testing and the actual amplitude of resonance during tests is unknown.

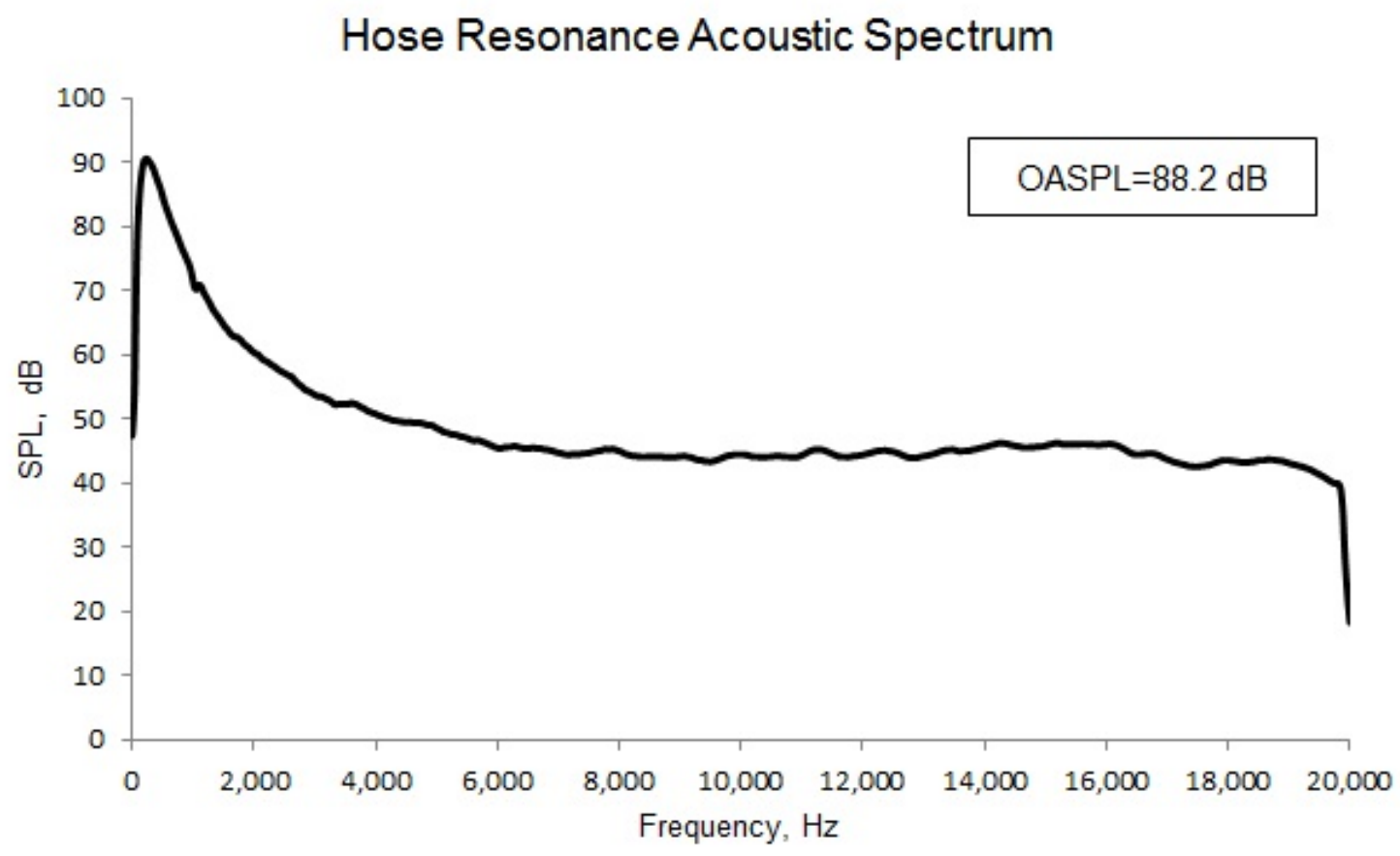

Figure 7.7: Acoustic resonance of the flexible hose

\subsection{Scale Model Baseline Acoustic Performance}

Thorough quantification of the scale model acoustics are undertaken to be able to make comparisons with the results stemming from configuration changes. All scale model microphone data is acquired at a frequency of $15,000 \mathrm{~Hz}$ for 30 seconds in order to capture the range of interest $-0-7,500 \mathrm{~Hz}$ - as discussed previously. The frequency bandwidth for these conditions is approximately $14.6 \mathrm{~Hz}$. Table 7.1 shows the microphone measurement locations in in the scale model. Two configurations are used given the limited number of microphones available. With the microphones in

\footnotetext{
${ }^{1}$ It was found that a smaller bandwidth provided no added benefit
} 
the first configuration, all Mach numbers of interest are tested. The microphones are then moved to the second configuration and all Mach numbers are re-tested.

Table 7.1: Microphones Locations in the Scale Model

\begin{tabular}{|c|c|c|}
\hline Microphone & Location & Configuration \\
\hline 1 & $\mathrm{~A}$ & \multirow{1}{*}{} \\
\hline 2 & $\mathrm{~B}$ & \multirow{2}{*}{1} \\
\hline 3 & $\mathrm{G}$ & \\
\hline 4 & $\mathrm{H}$ & \\
\hline 5 & $\mathrm{~J}$ & \\
\hline
\end{tabular}

\begin{tabular}{|c|c|c|}
\hline Microphone & Location & Configuration \\
\hline 1 & C & \multirow{2}{*}{} \\
\cline { 1 - 2 } 2 & F & \multirow{2}{*}{2} \\
\hline 3 & D & \\
\hline 4 & I & \\
\hline 5 & E & \\
\hline
\end{tabular}

The baseline acoustic performance varies significantly for different Mach numbers, and Mach numbers $0.1-1$ are tested in addition to the design Mach number. With ten measurements per Mach number, 110 SPL plots are required to fully describe the baseline acoustics. Obviously, they cannot all be included in this thesis. For brevity, only the results for Mach 0.9 are presented here. Figure 5.8 (microphone measurement locations) is repeated here for convenience. 


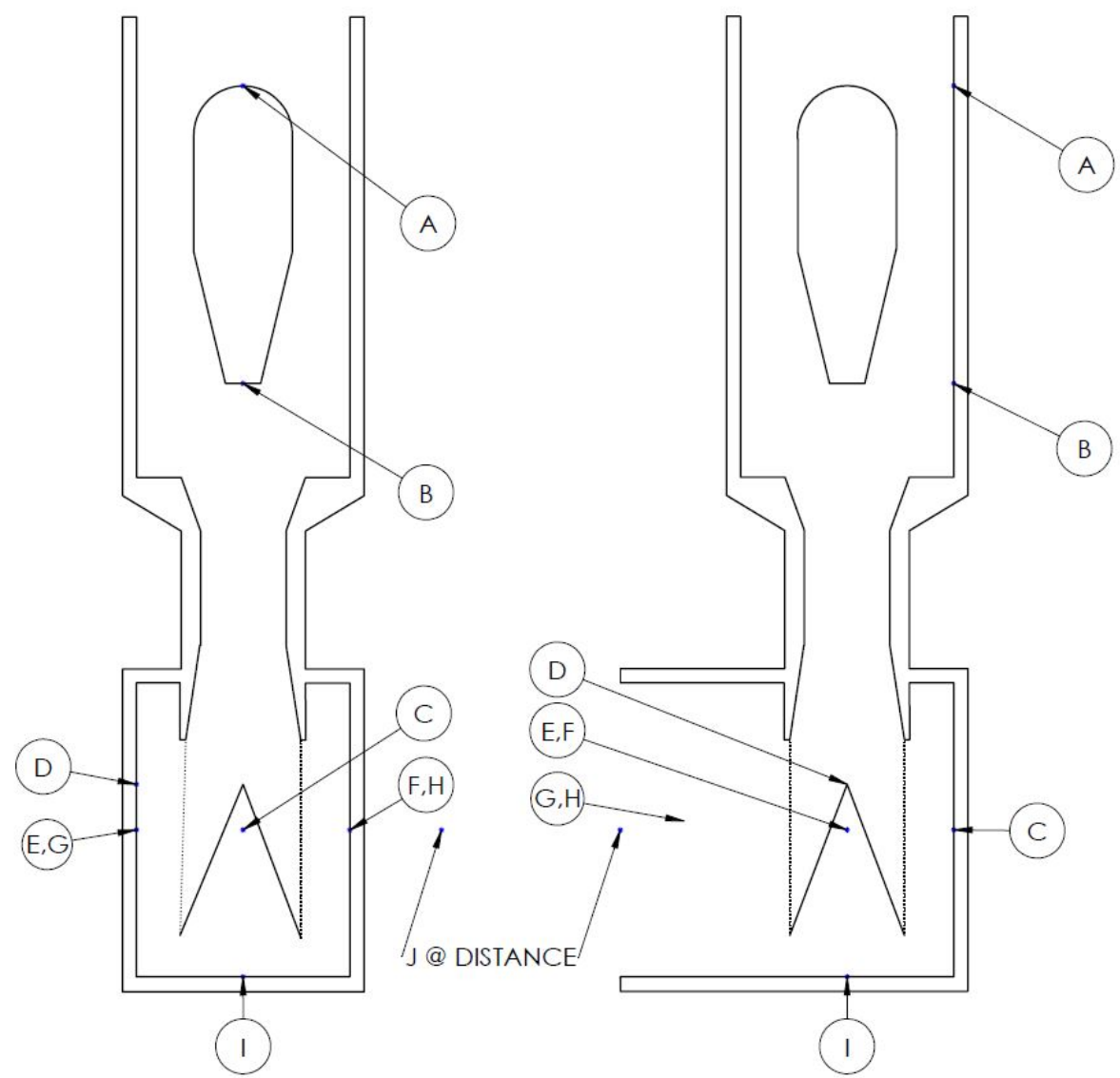

Figure 7.8: Repetition of figure 5.8: A front (left) and side (right) view schematic showing the microphone enumeration

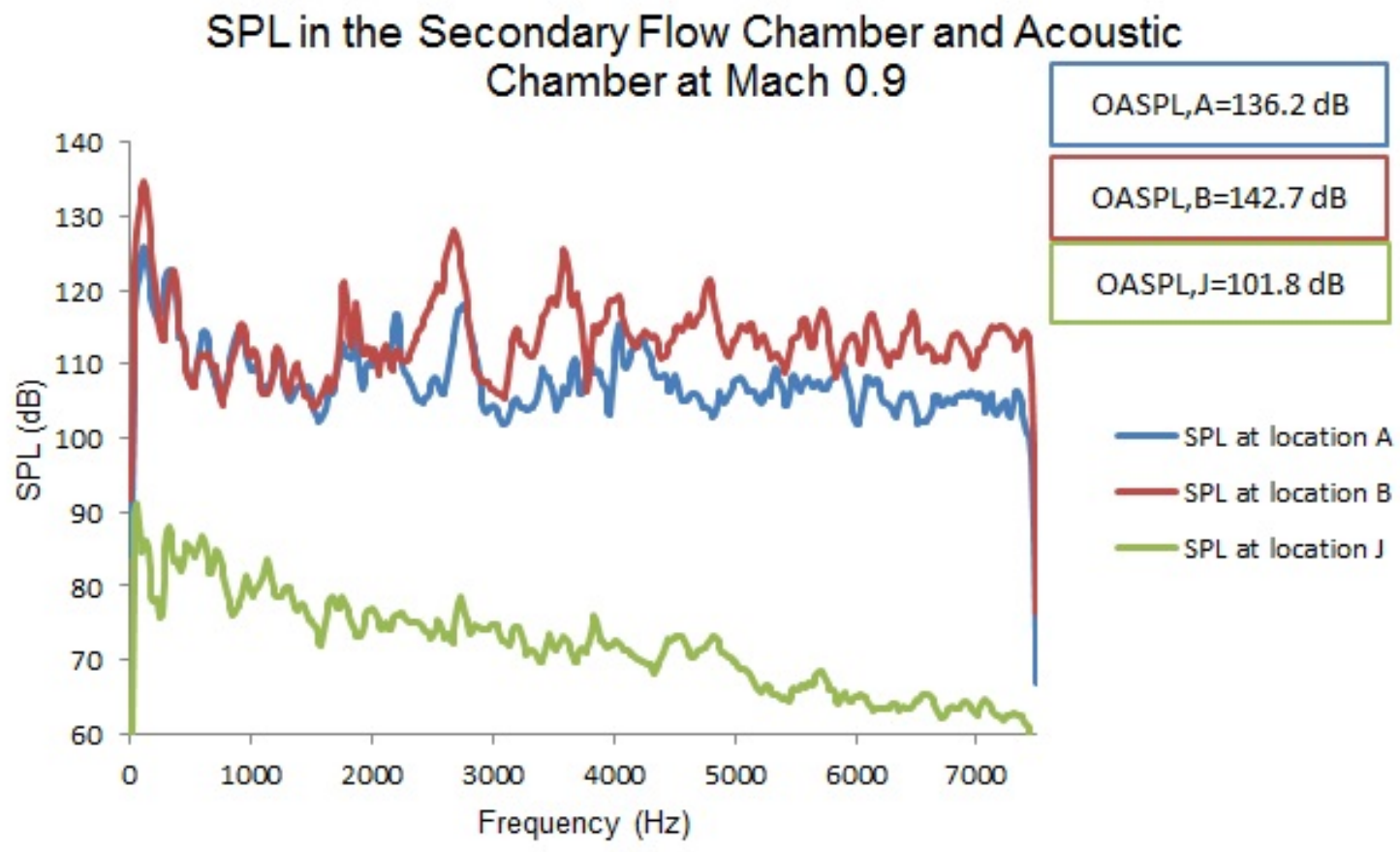

Figure 7.9: Acoustics in the secondary flow chamber \& anechoic chamber, baseline configuration, Mach 0.9 
There are a few general observations which can be made from the baseline acoustic performance. As can be seen in figure 7.9 , the primary nozzle is quite loud, and it is louder than the entrance to the scale model a short distance upstream. The noise at the entrance of the scale model is likely contaminated with primary nozzle noise. The spectrum of the noise perpendicular to the jet (location B) is quite different from that predicted in chapter 3 . The spectrum does not follow the self-similar behaviour typical of jet noise and the amplitudes are much higher than predicted, with a difference of approximately $50 \mathrm{~dB}$ at $100 \mathrm{~Hz}$. This is likely due to the confinement of the jet. Figure 7.9 also shows the acoustics at a distance (approximately 3 feet or 0.9 meters) from the scale model but still inside the anechoic chamber. As expected, these noise levels are the lowest of any measured, with an OASPL of 101.8 dB. The levels are still relatively high in comparison to the valve noise measured earlier. It can be deduced that the noise being generated in the chamber is much greater than the noise being generated outside the chamber (every $6 \mathrm{~dB}$ is double the sound pressure) and the likelihood of noise contamination is quite small due to the extreme noise levels being generated.

The noise measurements surrounding the silencer, referred to as lower outlet chamber noise, all appear to be rather similar, with different measurement locations $(\mathrm{C}, \mathrm{D}, \mathrm{E}$ and F) providing very similar acoustic results with the small peaks and valleys often being the same for the different locations. This is expected as the measurements are made all around the perforated cylinder at nearly equal distances from the perforated cylinder. This trend is valid for the upper outlet chamber measurements (locations $\mathrm{G}$ and $\mathrm{H}$ ) as well, although the measurements at this location have smaller amplitudes.

The obvious exception to the outlet chamber noise is for the measurements behind 


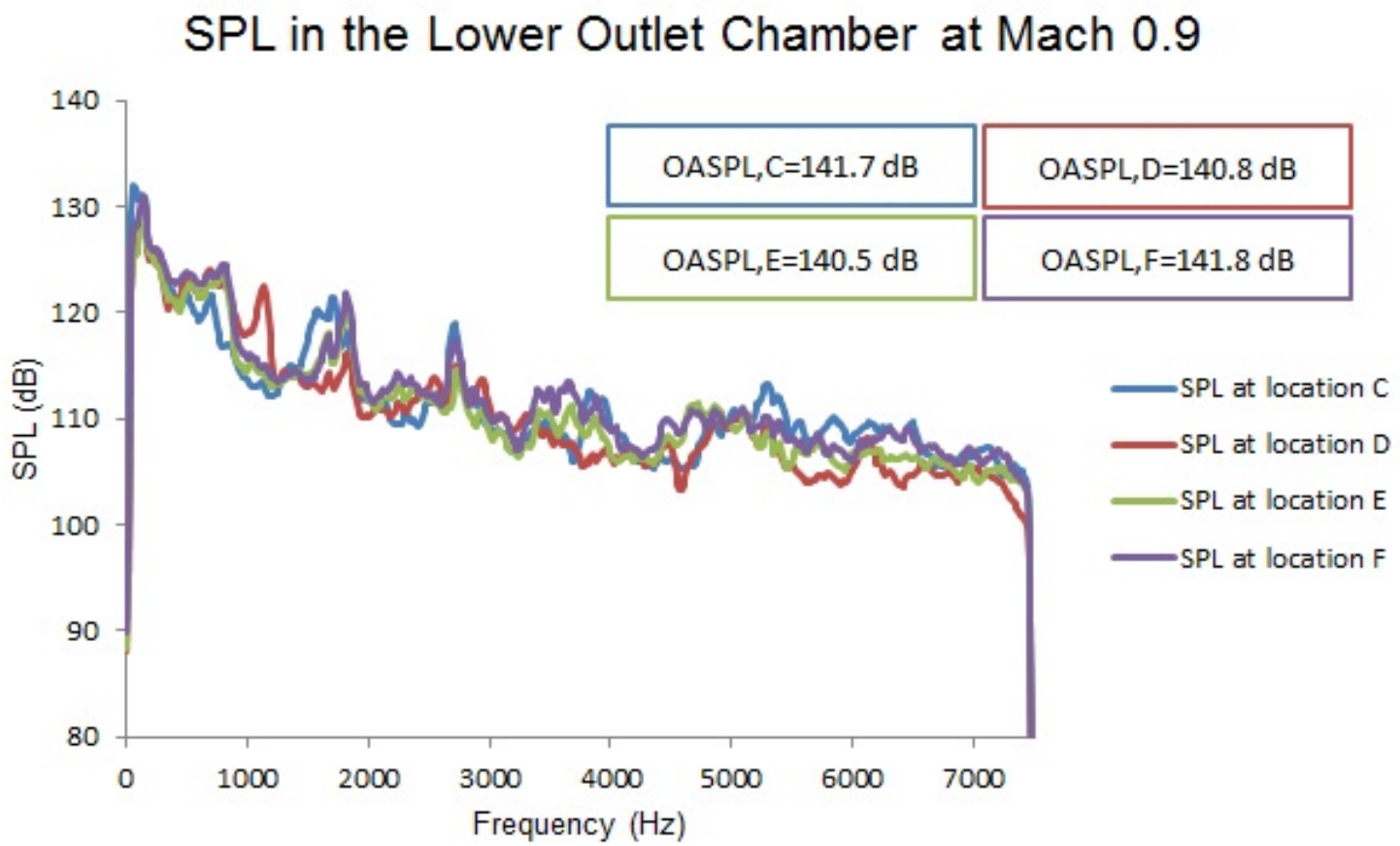

Figure 7.10: Acoustics in the lower outlet chamber, baseline configuration, Mach 0.9

the cone (location I). As can be seen in figure 7.12 the performance here is quite different from that seen in figure 7.10. At $144.4 \mathrm{~dB}$, this OASPL is the highest of any measurement location. The reason for this behaviour is believed to be due to the open rear of the cone and it is discussed in the next chapter.

An important finding not shown in figures 7.9 to 7.12 is the generation of discrete tones at certain Mach numbers. For the Mach number range of approximately 0.720.82 it is found that a tone is generated, and the frequency of the tone varies from 2593-2651 Hz (respectively) with an octave sometimes present. The tone can be seen in figure 7.13 , the noise measurement at position $\mathrm{C}$ at Mach 0.8. The frequency of the tone very closely matches the half transverse resonant frequency of the throat, $2694 \mathrm{~Hz}$, and the first azimuthal mode of the throat, $2589 \mathrm{~Hz}$. The prediction of jet preferred frequency in chapter 3 can be modified for this velocity range resulting in a 


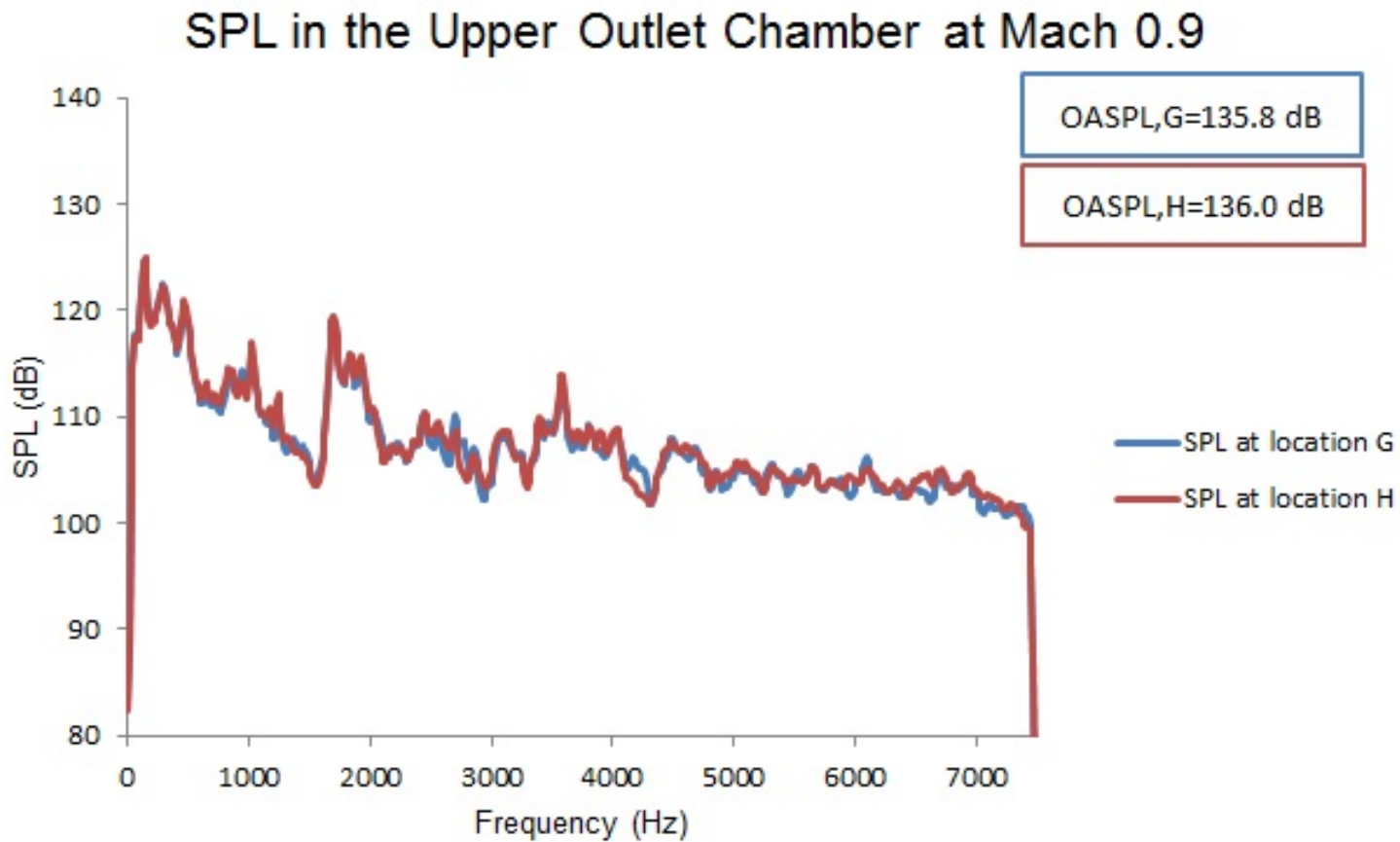

Figure 7.11: Acoustics in the upper outlet chamber, baseline configuration, Mach 0.9

jet preferred frequency ranging from $1872-2103 \mathrm{~Hz}$. Although this is lower than the observed tone, it is not terribly different, and the Strouhal number may shift leading to a coupling of the oscillations. The tone is typically captured by every microphone and not only at location C. Location B experiences the greatest magnitudes of this tone with a measured tonal frequency of $145 \mathrm{~dB}$ at Mach 0.8 . The microphone at location B actually saturates upon recording this tone (saturation occurs at \pm 1200 Pa or $155.6 \mathrm{~dB}$ ) therefore the true magnitude of the tone is greater than $146 \mathrm{~dB}$ but cannot be measured with the equipment available.

\subsubsection{Modal Decomposition}

The sound pressure level plots at first appear almost chaotic and unorganized, however, modal decomposition - that is an analysis of each spike observed on the SPL plot 


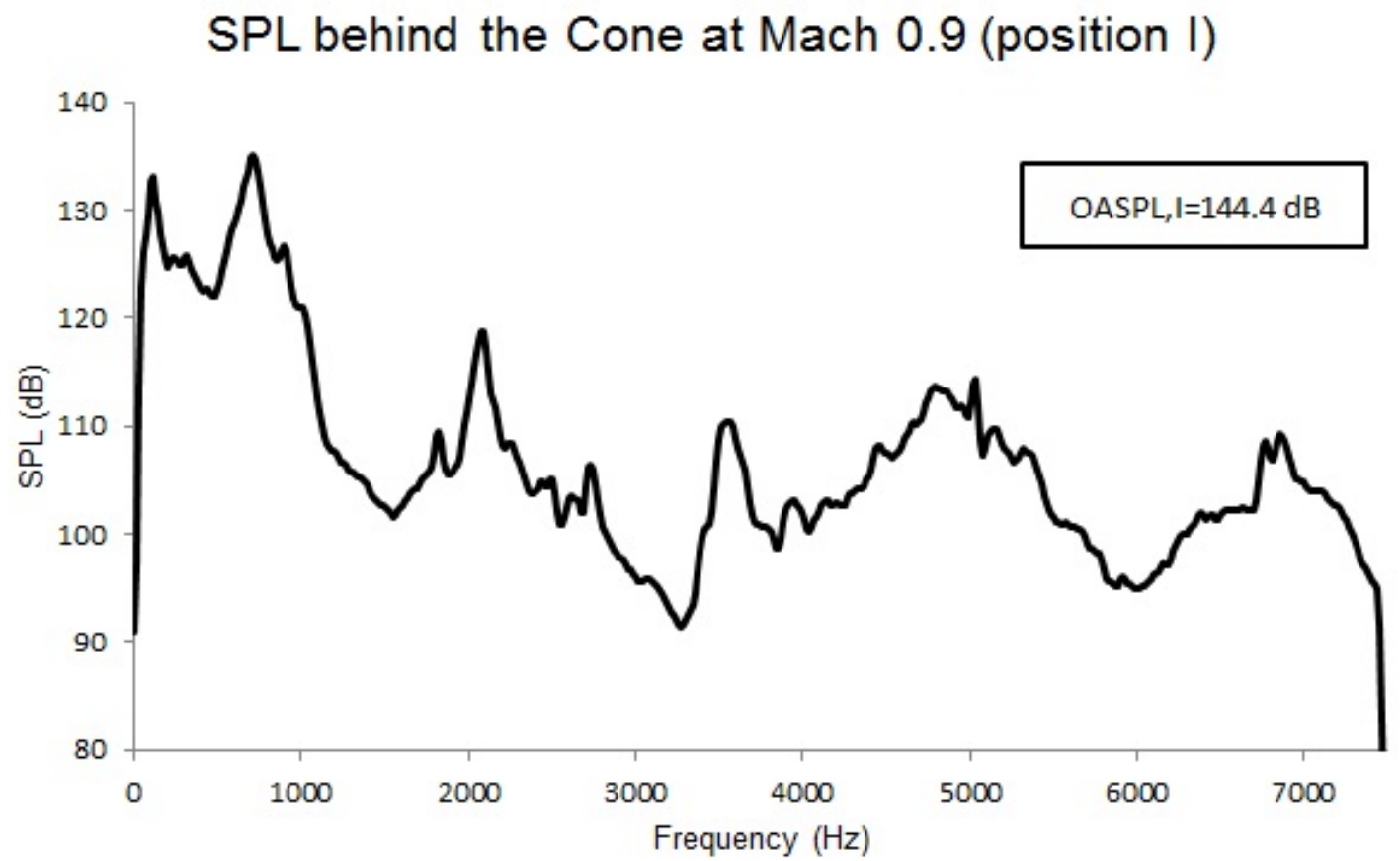

Figure 7.12: Acoustics behind the cone, baseline configuration, Mach 0.9

- can help clarify the noise spectrum. The predictions from chapter 3 are compared to the experimental acoustics in an attempt to better understand the spectrum. Figure 7.14 presents the modal decomposition at measurement location $\mathrm{F}$ showing that most peaks observed are clearly due to certain acoustic modes of the ejector. An interesting observation is that frequencies which a higher density of natural modes show greater acoustic levels than the frequencies with single modes present. The higher frequencies are not analyzed as they go above the Schroeder frequency and involve high octaves and possible combinations of natural modes. Though omitted here for brevity, the behaviour in the inlet flow chamber is found to exhibit different peaks but of similar magnitude. Many of these peaks are attributable to the throat natural modes implying that the noise generated in the throat has a tendency to propagate better upstream (perhaps due to the perforated cylinder attenuating some noise). 


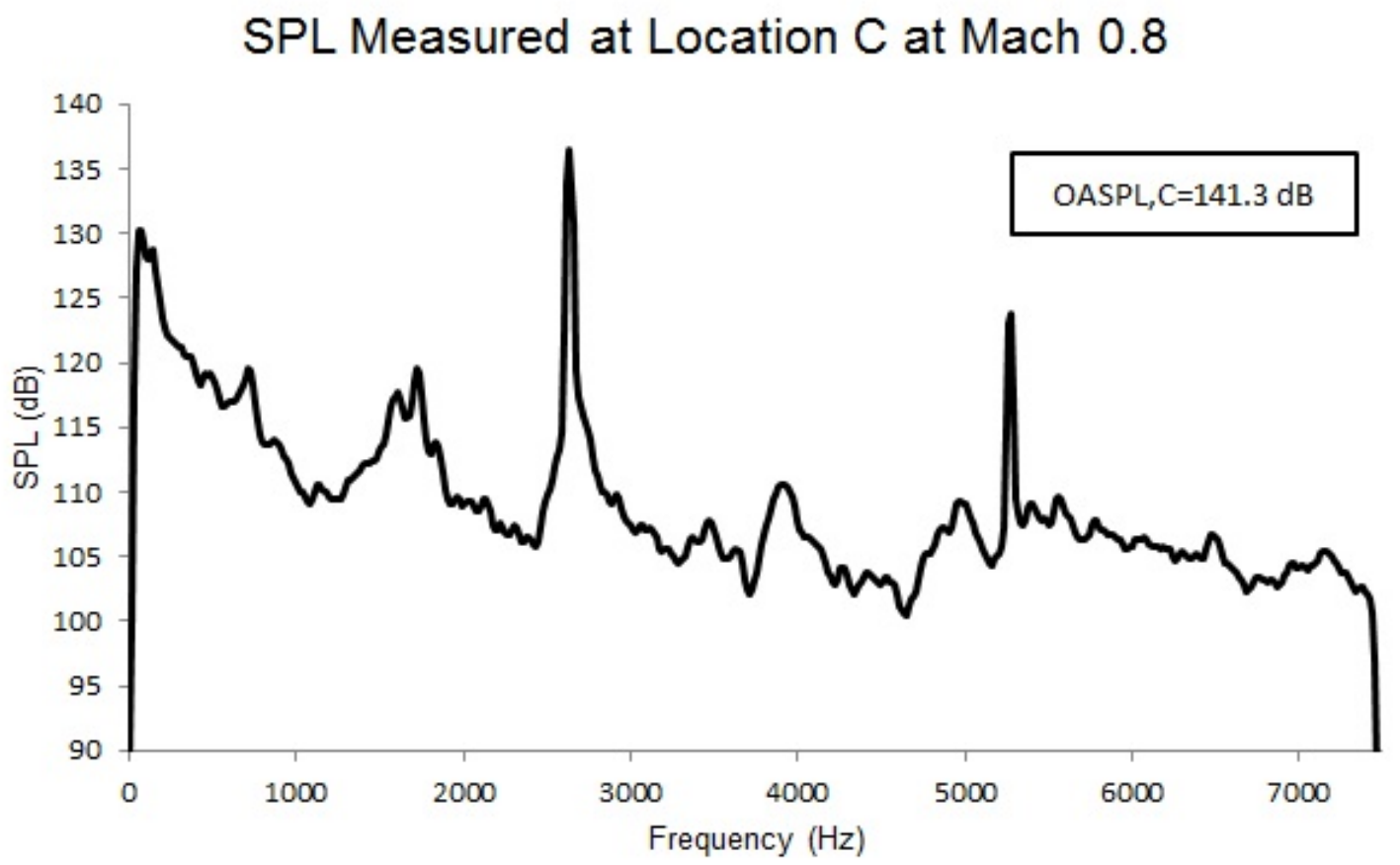

Figure 7.13: The acoustic signature at location $\mathrm{C}$ at Mach 0.8 showing a clear tone and its octave 


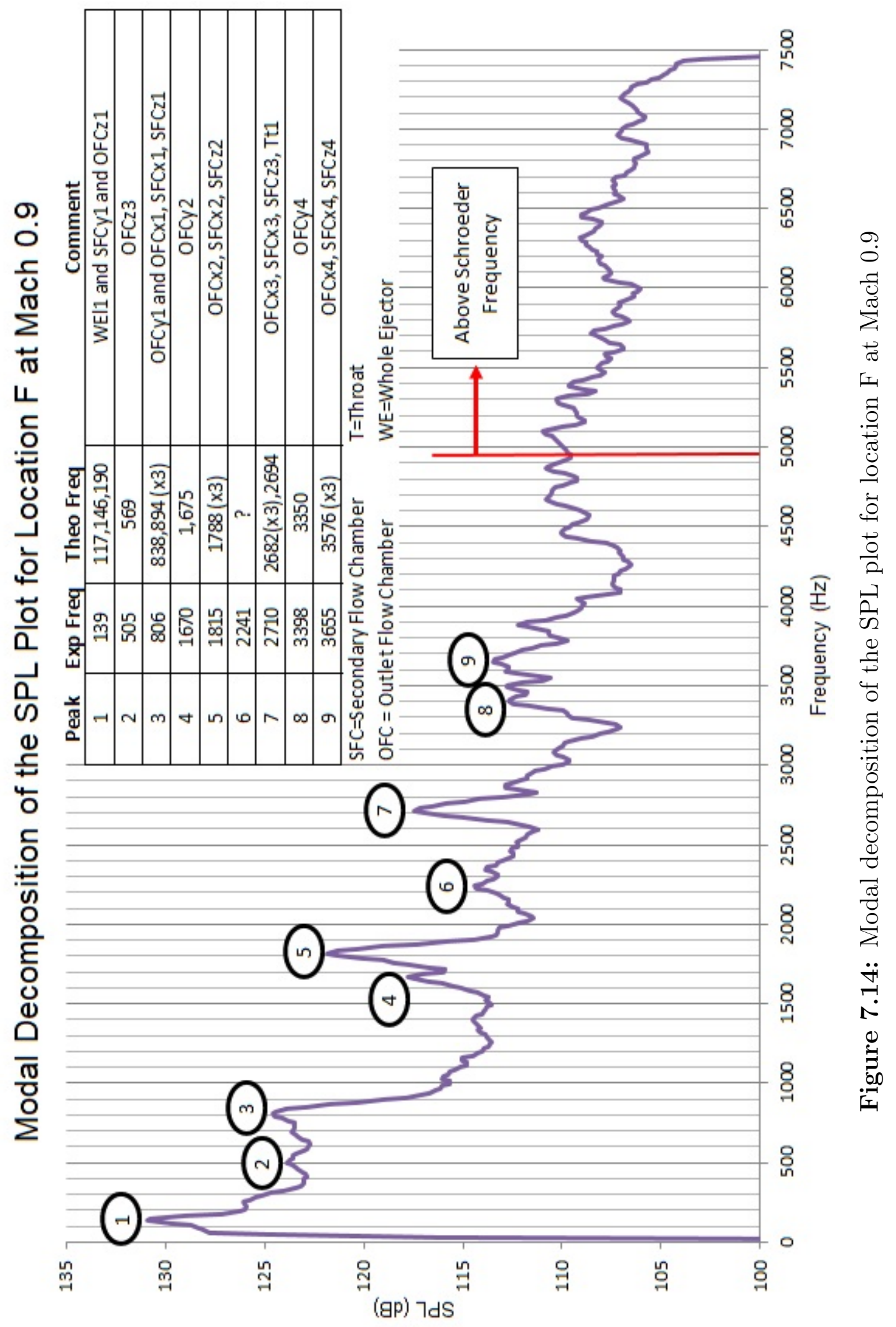




\subsection{Scale Model Baseline Aerodynamic Perfor- mance}

To evaluate the aerodynamic performance of the scale model, the same pressure measurement system data is recorded as for chapter 6. To measure the flow velocity and uniformity of the ejector model, the Pitot-static probe is traversed in different locations and directions. Unfortunately, the research group has no automatic traverse available thus the traverse is performed by repeating many runs and moving the Pitotprobe by a small increment for each run. The traverses are performed downstream of the inlet, downstream of the primary nozzle and slightly upstream of the outlet, as depicted in figure 7.15. An $\mathrm{x}-\mathrm{y}-\mathrm{z}$ coordinate system is established for the traverses, matching the coordinates outlined in chapter 3 .

It should be noted that the primary nozzle prevents a full $\mathrm{x}$ direction traverse at the inlet of the model. In addition, for the inlet traverses the tip of the Pitot-static probe is quite close to the perforated plate thus the flow is likely not fully developed. The velocities obtained from each traverse of the secondary flow chamber inlet and for the outlet chamber are normalized by the average velocity of the given traverse. The primary nozzle Mach numbers are normalized by the target Mach number. The distances are also all normalized from 0 to 1 , with 1 representing the wall to wall distance 2 . The Pitot-static probe is not long enough to traverse the entire wall to wall distance. The traverse results are shown in figures 7.16 and 7.17 . It is worth mentioning that the results found in figure 7.17 have an uncertainty above $1000 \%$ due to the low velocities being measured with large range pressure transducers.

There are a few observations worth noting: The increment used in moving the

\footnotetext{
${ }^{2}$ Values normalized to protect intellectual property
} 


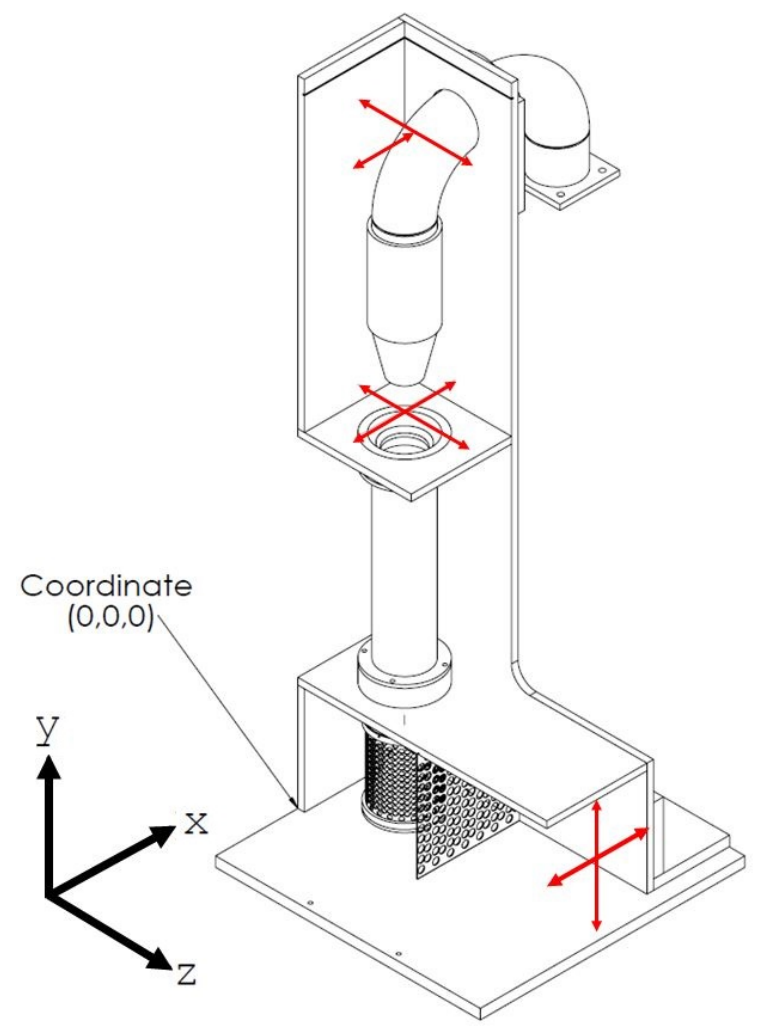

Figure 7.15: The locations and directions of the traverses in the scale model with the coordinate system defined

Pitot-static probe appears to be too small to properly resolve the primary nozzle jet profile. The model scale means that the size of the Pitot-static probe is nonnegligible and a certain amount of error undoubtedly results due to this. The z direction traverse downstream of the primary nozzle shows a point with a normalized Mach of approximately 0.3 . It was observed that at this point the probe was in fact aligned with the edge of the primary nozzle. The outlet $\mathrm{x}$ direction traverse shows a significant dip in the center, corresponding to the location of the collector upper lip. It would be expected that the collector upper lip will cause a velocity stagnation point thus this trend is not unexpected. The outlet y direction traverse shows uniformity in the center with a velocity increase near the wall. The reason for this effect is unknown but the gap between the outlet chamber and the collector may cause this region to 


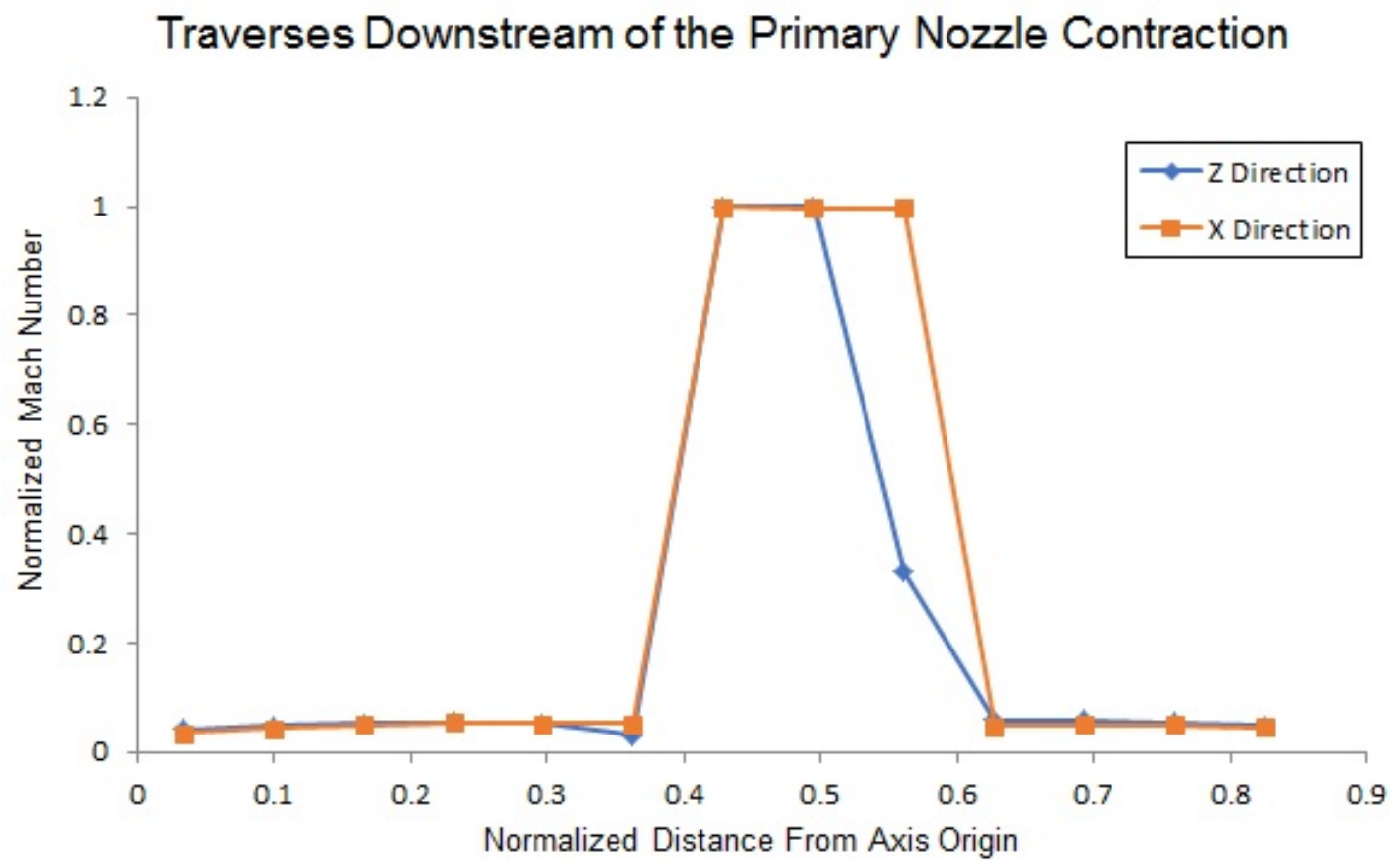

Figure 7.16: Traverse results downstream of the primary nozzle
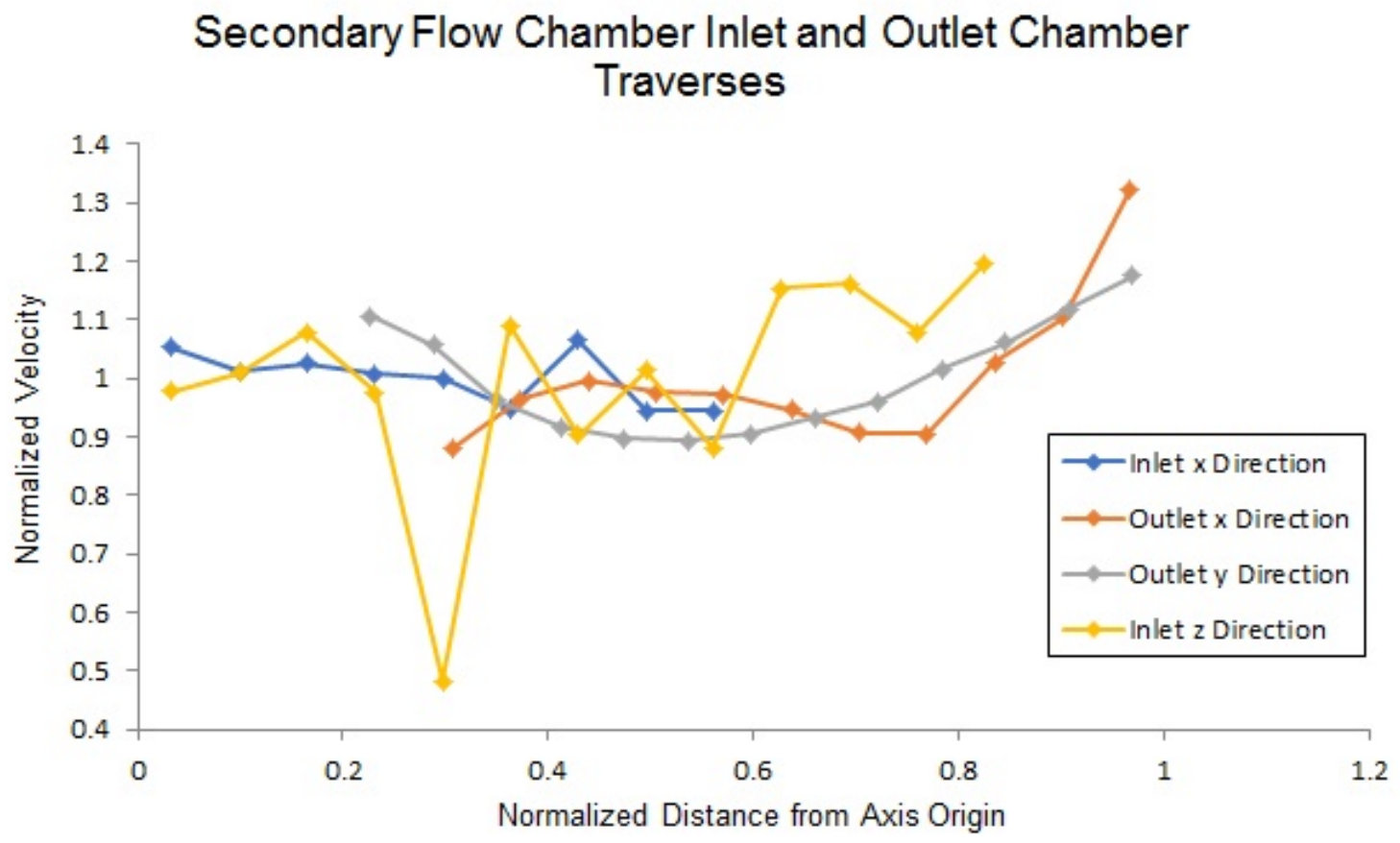

Figure 7.17: Traverse results at the scale model inlet and outlet 
entrain air into the collector, essentially acting as an ejector.

The inlet velocity appears to fluctuate but the magnitude of said fluctuations is small. The velocity distortion, a percentage measure of the variation of airflow over a given plane oriented perpendicular to the ejector walls, can be used to quantify these fluctuations. The distortion can be calculated from equation 7.2 :

$$
u_{\text {dist }}=\frac{u_{\max }-u_{\min }}{u_{\text {avg }}} * 100 \%
$$

where $u_{\text {dist }}$ is the velocity distortion, $u_{\max }$ is the maximum measured velocity, $u_{\min }$ is the minimum measured velocity and $u_{a v g}$ is the average measured velocity. The resulting velocity distortion for the combined inlet $\mathrm{x}-\mathrm{z}$ plane traverses is $72.8 \%$. Looking at the inlet traverse in the $\mathrm{z}$ direction, a point can be seen with a normalized velocity near 0.5. This large deviation was later found to be due to glue blocking a small portion of the inlet perforated plate. The glue was removed and the flow became more uniform. If this point is neglected in the velocity distortion calculation, the resulting velocity distortion would drop nearly $50 \%$ to a value of $31.4 \%$.

Another important performance parameter is the entrainment ratio, mentioned in chapter 1. It can be calculated from equation 1.1, repeated here for convenience:

$$
E R=\frac{\dot{m}_{\text {primary }}}{\dot{m}_{\text {secondary }}}
$$

The entrainment ratio is a function of primary nozzle velocity and it increases with increasing velocity. The entrainment ratio for the scale model at the design Mach number is found to vary between 1.44 and 1.58 depending on the calculation method employed (location of measured velocity, averaging technique used, area approximation etc.). 


\subsubsection{Temperature Trend}

In order to estimate the temperatures encountered, a thermocouple is secured to the outside wall of the primary nozzle. The temperature measurement system measures this temperature (in degrees Celsius) along with the time and the ambient temperature in the HSWT facility. The resulting drop in primary nozzle temperature for a long duration test at the design Mach number is shown in figure 7.18. Acoustic measurements tests require a minute or less of runtime thus the resulting temperature drops are expected to be much lower than the recorded $13^{\circ}$ drop. While the speed of sound and thus the acoustic power emitted by the jet do vary with temperature, the small temperature drop is deemed negligible for normal acoustic data capture runs. In addition, there is currently no way of controlling the jet temperature with the given facility.

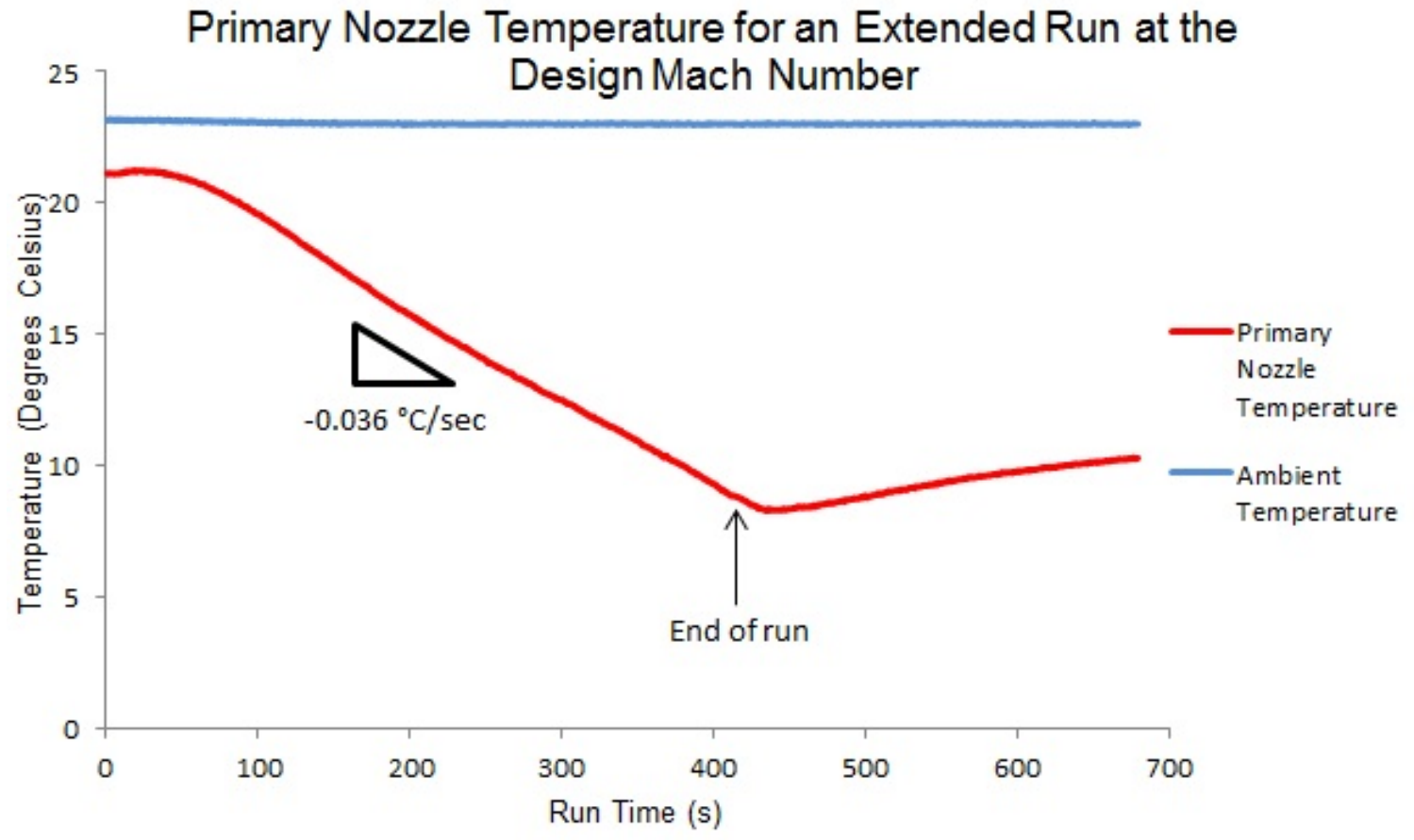

Figure 7.18: The measured temperature of the primary nozzle wall and ambient for a long duration run at the design Mach number 


\section{Chapter 8}

\section{Results II: Silencer Modifications}

This chapter outlines the acoustic performance obtained through various modifications to the silencer. From its onset, this project was defined as an investigation into the impact that the cone may have on the ejector acoustics thus the cone is the primary focus of this work. Intuitively, changing the angle of the cone is one of the first tests which should be undertaken, however, this was tested by a third party thus it is not repeated in this work. All results in this chapter are recorded using the same parameters as the baseline tests and for Mach numbers $0.5-1$ in intervals of 0.1 as well as at the design Mach number. For brevity, only the results for the design Mach number at microphone measurement location $\mathrm{F}$ are presented (along side the baseline results for easy comparison). The acoustics measured at the primary nozzle (measurement location B) are important for certain applications so interesting findings at measurement location B are discussed. Each modification is briefly introduced before presenting the results and discussing. Figure 5.8 (microphone measurement locations) is repeated here for convenience.

As is mentioned in chapter 2, the velocity of the jet issuing from an ejector has an important impact on the noise generation. The ejector used has a constant area outlet and the primary nozzle has a constant mass flow for a given Mach number, 


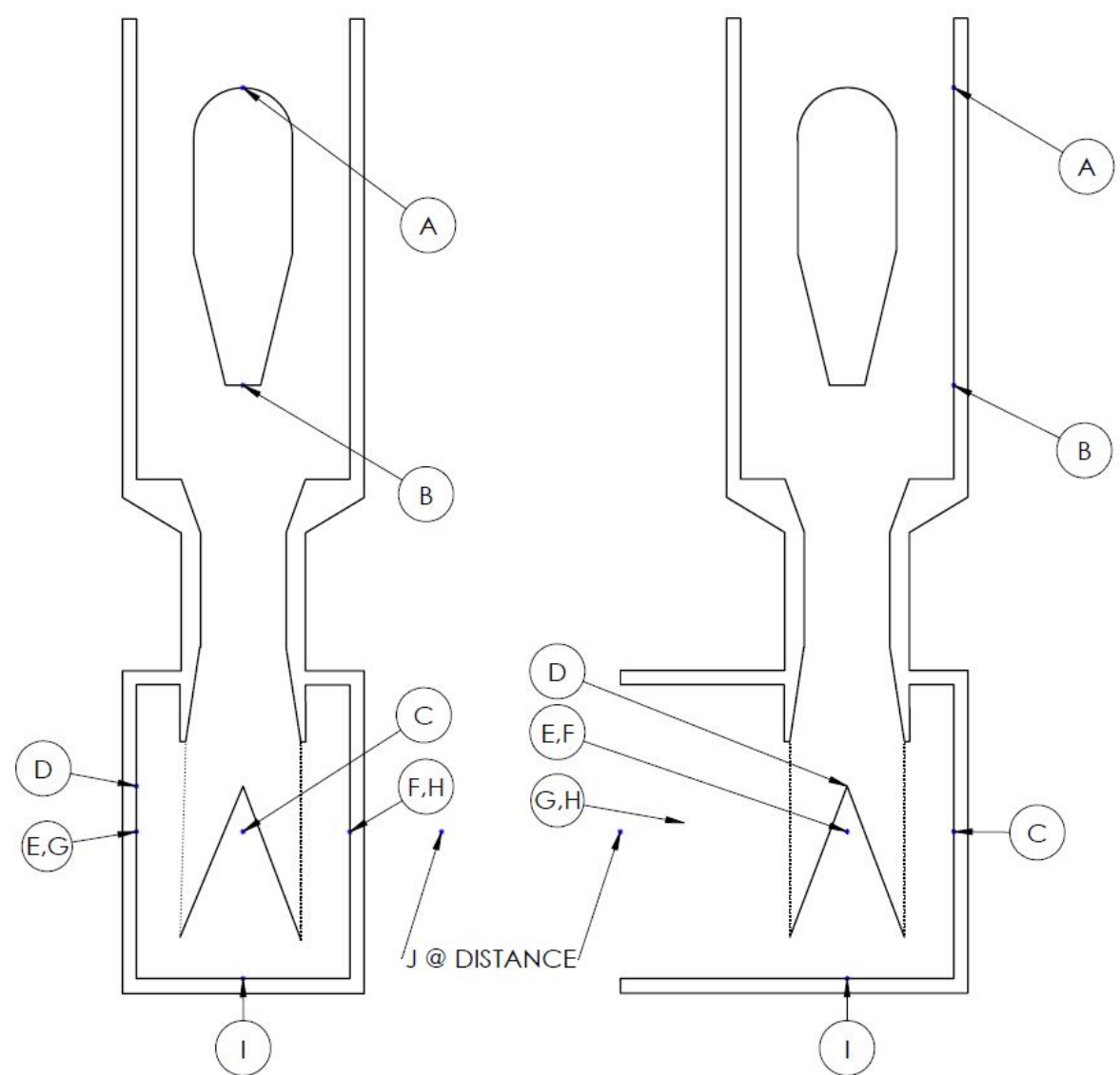

Figure 8.1: Repetition of figure 5.8: A front (left) and side (right) view schematic showing the microphone enumeration

therefore, any change in outlet velocity can come only from a change in entrainment ratio. For a silencer configuration which causes a pressure drop larger than that of the baseline, the entrained airflow, thus total mass flow, thus outlet velocity, thus noise will be less. It is important to try and isolate a decrease in noise levels from a decrease in flow velocity.

Although the entrainment ratio is not measured for every test, it can be estimated using the secondary flow chamber static pressure. The entrainment ratio is estimated using the assumption that the total pressure changes proportionally with static pressure. The Mach number can be isentropically calculated from the square root of the 
total to static pressure ratio (see equation 4.2 thus the change in entrainment ratio can be calculated from the square root of the static pressure ratios, that is

$$
E R_{\text {new }}=\sqrt{\frac{P_{\text {new }}}{P_{\text {baseline }}}} E R_{\text {baseline }}
$$

where the subscript new represents a new test configuration, and the subscript baseline represents the baseline configuration. Using this estimator along with a baseline entrainment ratio of 1.484 , the resulting entrainment ratio for new configurations can be estimated and used to qualitatively judge whether a change in acoustics is due to improved silencer performance or simply decreased mass flow. For configurations with worsened acoustics the entrainment ratio is not always discussed.

\subsection{Cone Surface Roughness}

A primary focus of the Carleton Aeroacoustics group is turbulent boundary layer noise thus the acoustic impact of the boundary layer forming on the cone is investigated. A series of cones shells are made from sandpaper of different grits (roughness) and then each is placed over the solid cone and tested, as different roughness's will change the boundary layer. In order to investigate extreme roughness's two cones are fabricated with small rocks glued to them. Figure 8.1 is a photo of the cones tested. 


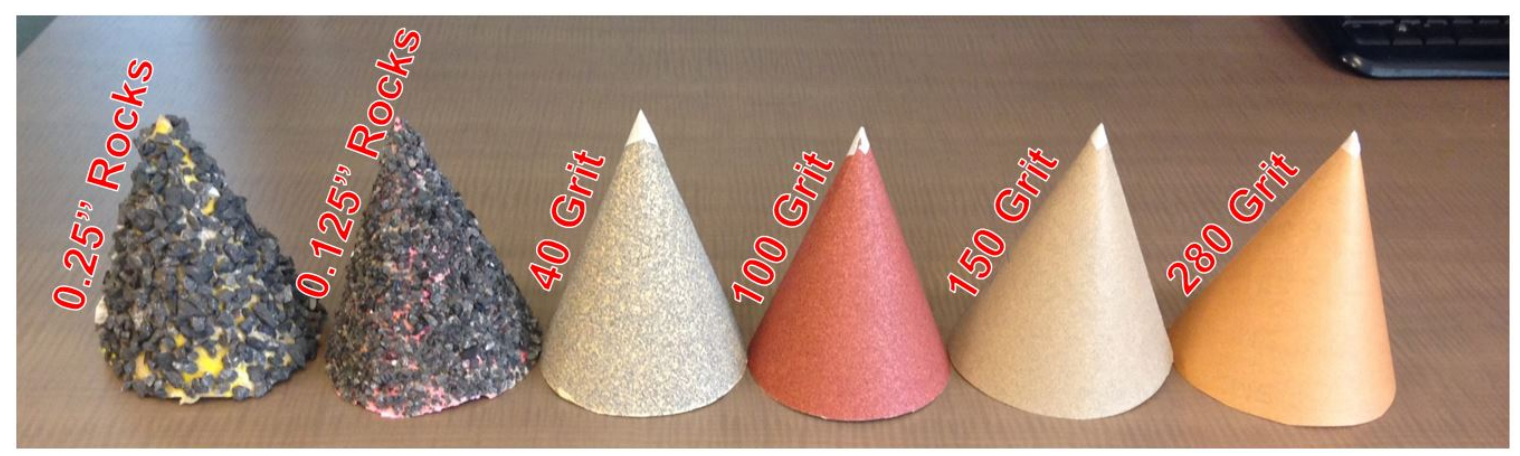

Figure 8.2: The cones used to test the acoustic impact of surface roughness

The grits and rock sizes used, along with the average particle size of each can be seen in table 8.1.

Table 8.1: The Cone Roughness's Tested and Their Average Particle Size \begin{tabular}{r|cccccc} 
Grit: & 280 & 150 & 100 & 40 & $0.125 "$ Rocks & $0.25 "$ Rocks \\
Average Particle Size: & $52 \mu \mathrm{m}$ & $92 \mu \mathrm{m}$ & $140 \mu \mathrm{m}$ & $425 \mu \mathrm{m}$ & $3.2 \mathrm{~mm}$ & $6.4 \mathrm{~mm}$
\end{tabular}

The resulting SPL spectrum at measurement location B show no difference for any of these cones. The SPL spectrum for measurement location $\mathrm{F}$ is shown in figure 8.3 .

As can be seen, the resulting noise is quite similar to that of the baseline for all roughness's. The difference between different roughness's comes in the valleys. For the low frequency valley between 1000 and $2000 \mathrm{~Hz}$ the rougher the cone, the smaller the SPL. For higher frequencies, above approximately 5000, this trend seems to reverse and the rock cones are found to increase the SPL. The peaks show nearly no difference for varying grits. The impact of cone surface roughness is deemed negligible given that the noise spectrum is dominated by large peaks. These peaks are likely caused by the natural modes predicted in chapter 3 and presented in the modal decomposition. If a different silencer configuration successfully decreases these 


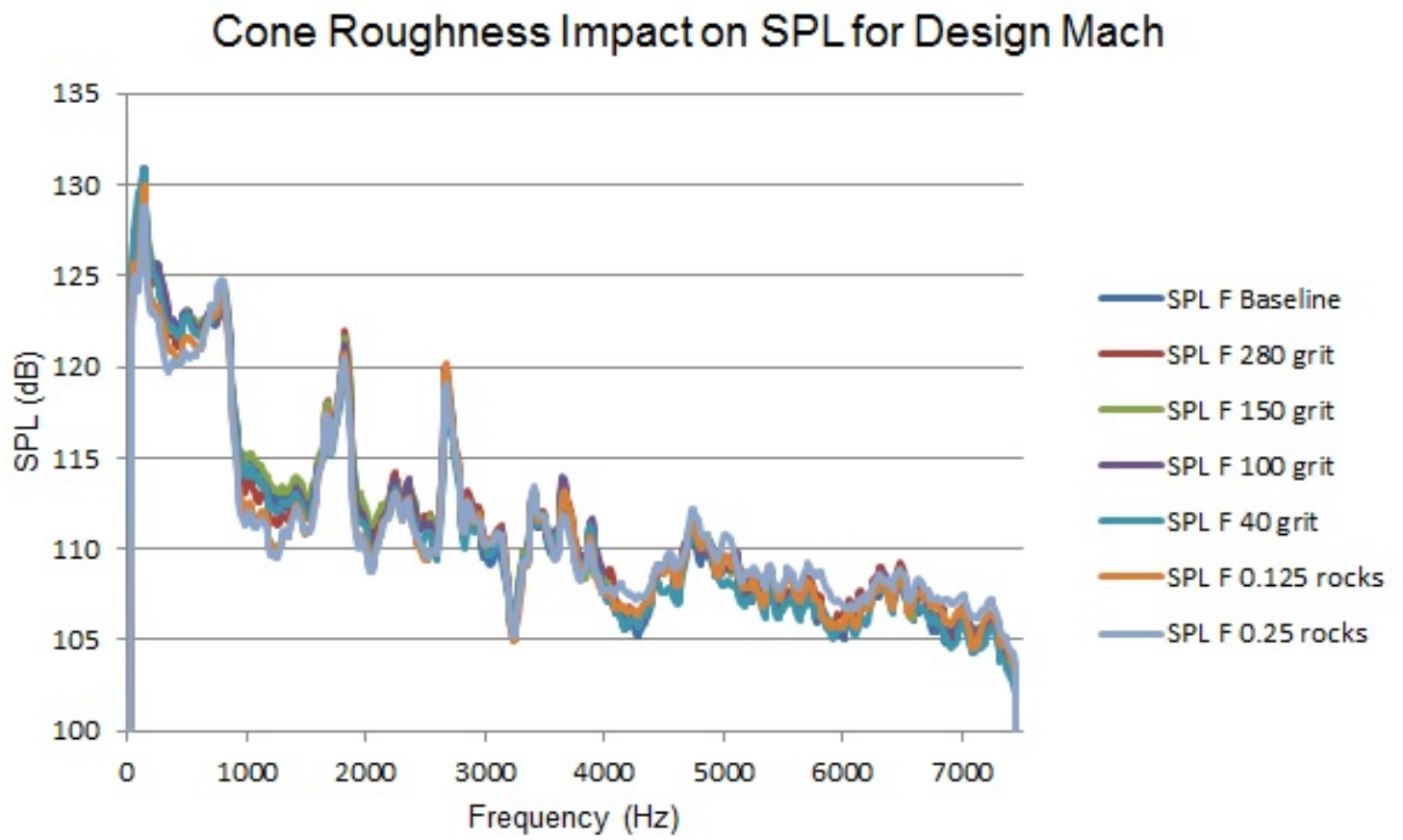

Figure 8.3: The SPL spectrum as measured at location $\mathrm{F}$ for all surface roughness cones

peak magnitudes, such that the spectrum follows the noise levels found in the valleys, cone roughness may be worth reinvestigating. The differing performance for different grits may also motivate an investigation into a fractal roughness.

\subsection{Closed Cone Rear}

Given that the highest noise levels experienced in the baseline configuration are found behind the cone, an analysis on the impact of closing the rear of the cone is undertaken. The cone is closed with a thin (0.125 inch or $3.2 \mathrm{~mm})$ acrylic plate and the interior of the cone is filled with acoustic foam in order to prevent resonance from occurring. The difference in the acoustics for this configuration with respect to the baseline, as measured at location I, can be seen in figure 8.4 . 


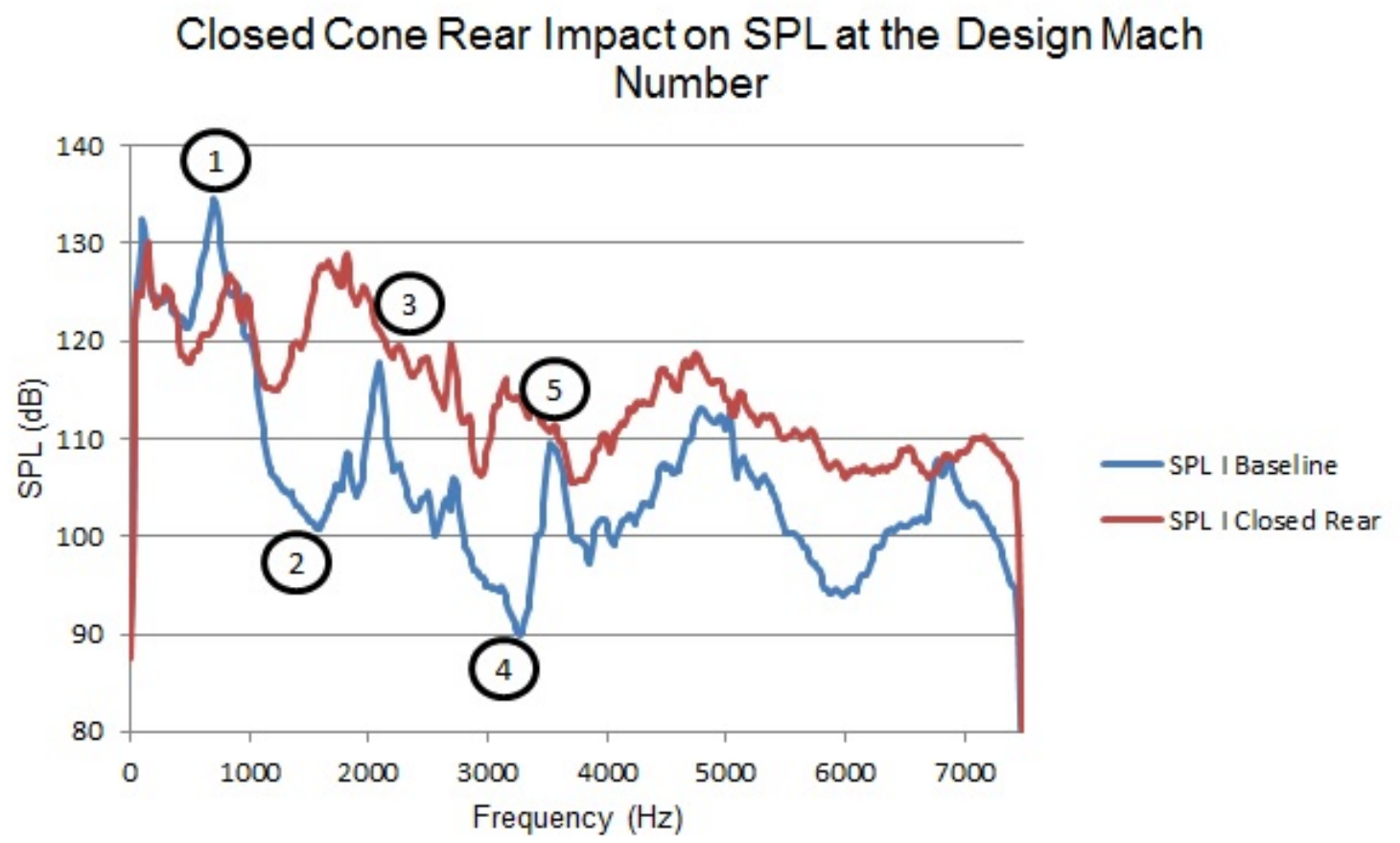

Figure 8.4: The SPL spectrum as measured at location I for the cone with a closed rear

Initially it was believed that the open rear of the cone provides a location where flow could recirculate causing acoustic mode excitation, however, upon further investigation it is believed that the open cone acts as a Helmholtz resonator. The open cavity contains a certain volume of air and the small space between the cone and base assembly acts as a circumferential neck through which the large volume can oscillate. The resonance frequency of a typical Helmholtz resonator, $f_{\text {Helmholtz }}$, can be estimated using formula 8.2 ;

$$
f_{\text {Helmholtz }}=\frac{c}{2 \pi} \sqrt{\frac{S}{v_{\text {Helmholtz }} \alpha}}
$$

where $S$ is the cross sectional area of the neck, $v_{H e l m h o l t z}$ is the volume contained within the resonator and $\alpha$ is the length of the neck. The estimates for $S, v_{\text {Helmholtz }}$ and $\alpha$ for this situation are obtained in the manner presented in figure 8.5. Using 
equation 8.2 and these estimators gives a Helmholtz resonance of $694 \mathrm{~Hz}$. For the

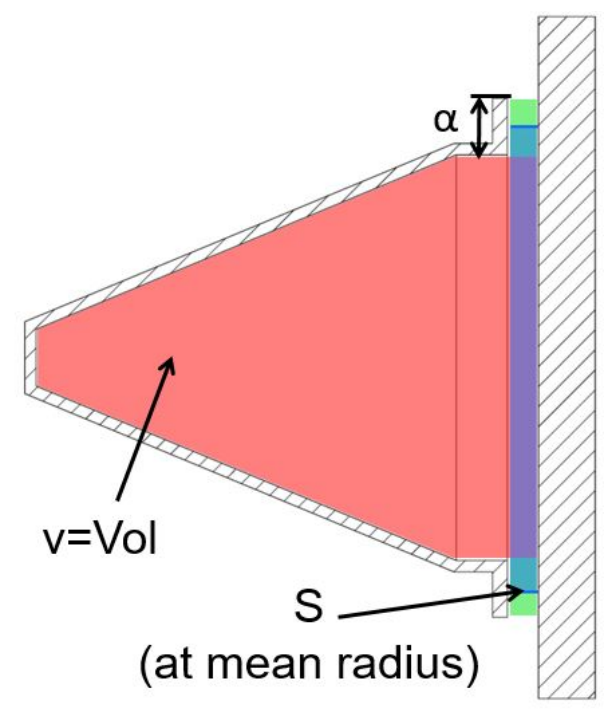

Figure 8.5: The estimation used for $S, V$ and $\alpha$

baseline SPL plot in figure 8.4, distinct peaks and valleys are observed. The first peak is present for both configurations thus it can be attributed to something else, likely the whole ejector longitudinal mode. The peak labelled 1, at $703 \mathrm{~Hz}$ is very near the estimated Helmholtz resonance of $694 \mathrm{~Hz}$ (and shows no indication that the natural acoustic modes of 838 and $894 \mathrm{~Hz}$ are excited). The subsequent peaks labelled 3 and 5 happen at 2167 and $3604 \mathrm{~Hz}$ respectively. The odd numbered integer frequency multiple suggests the higher frequency peaks are octaves of the fundamental. In addition, there are valleys, labelled 2 and 4, which occur at 1567 and 3267 $\mathrm{Hz}$ respectively. These are somewhat near to even numbered multiples of the fundamental. The closed rear configuration helps understand the phenomenon occurring, however, this configuration is louder than the open cone thus an open cone is used for subsequent tests. 


\subsection{Fin Cone}

In an attempt to reduce or eliminate any helical oscillation which may occur inside the ejector silencer (there is a possibility that impingement onto the cone may lead to helical oscillations) a special cone with fins is $3 \mathrm{D}$ printed from ABS plastic, shown in figure 8.6 .

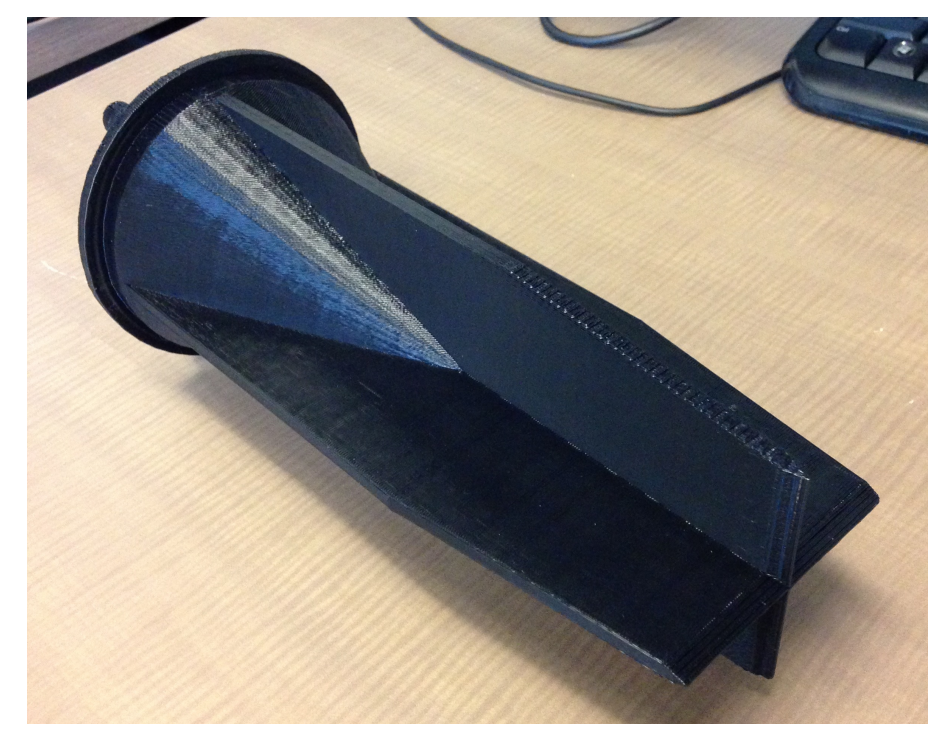

Figure 8.6: The 3D printed cone with fins

The number of fins is arbitrarily chosen as 4 . The fins are $1 / 4$ inches $(6.4 \mathrm{~mm})$ wide, nearly flush to the wall of the perforated cylinder and they extend all the way to the diffuser inlet following the profile of the diffuser. The tips of the fin form a symmetric $45^{\circ}$ apex, the same as the cone angle. The fins are not rotationally constrained with respect to the throat and two configurations are tested: with the fins parallel to the walls of the ejector and with the fins at $45^{\circ}$ to the walls of the ejector. Poor acoustic performance of these configurations leads to the fins being cut such that they do not extend past the apex of the cone, resulting in fins with a blunt tip.

The noise at measurement location B for both long-fin configurations is found to 
be quite close to that of the baseline, although the two lowest frequency spikes are found to be reduced by 7 and $5 \mathrm{~dB}$ respectively. The noise at location $\mathrm{F}$ for all three configurations is shown in figure 8.7 .

\section{Impact of 4 fins extending from the cone into the diffuser entrance}

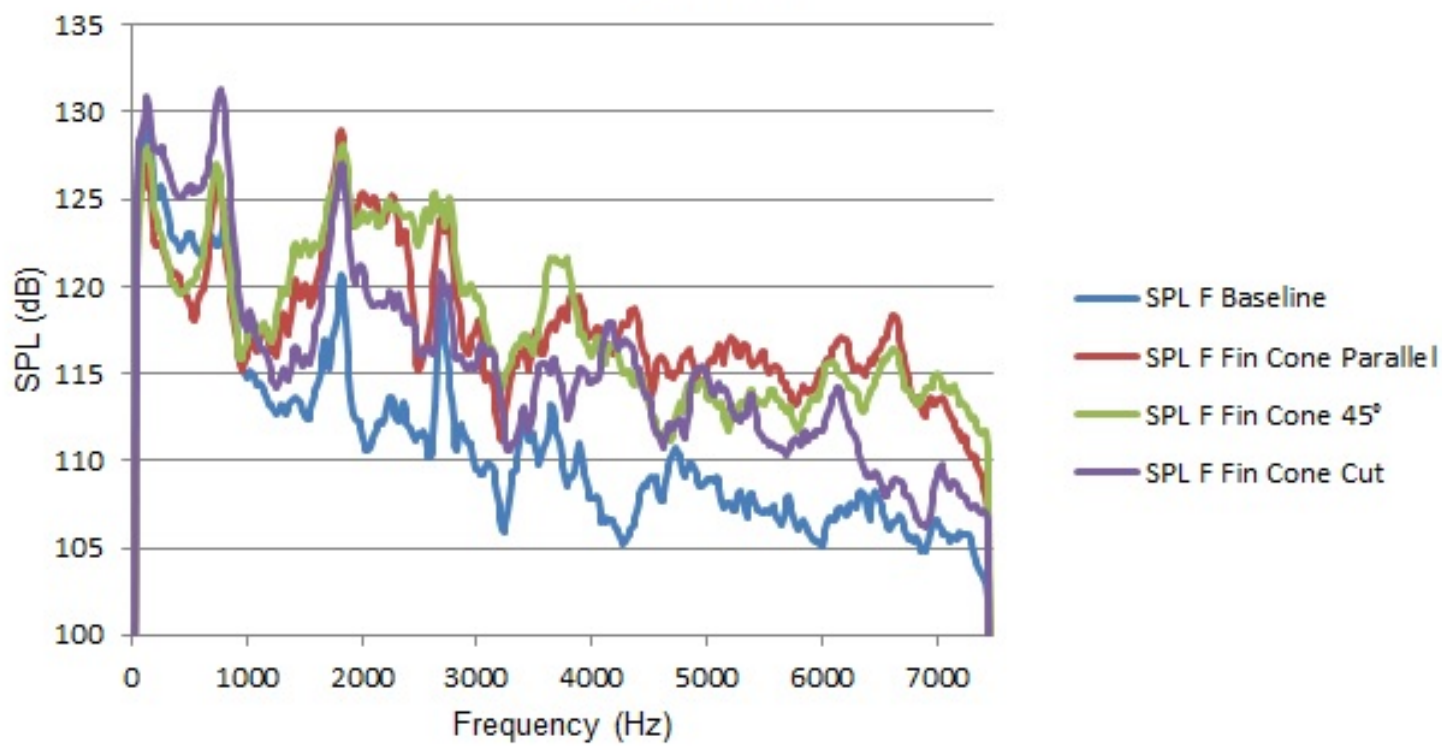

Figure 8.7: The SPL spectrum as measured at location F for all fin cone configurations

Both with the fins parallel to and at $45^{\circ}$ to the walls it is found that the resulting noise at location $\mathrm{F}$ is undesirable, that is that the noise generated by this configuration is greater than the baseline configuration. A possible explanation for the increase in acoustic levels is that the fins may cause excessive boundary layers to form thus causing a flow blockage which in turn causes an increase in velocity (thus noise). This idea has not been investigated further, however, it is the reason for testing the "cut fins" configuration.

It is also found that a large increase in frequency is observed at 761 and 1816 $\mathrm{Hz}$, approximately corresponding to the first and second natural acoustic modes of 
the outlet flow chamber. The reason for this spike is unknown but may be due to the proposed velocity increase causing greater excitation energy in the outlet flow chamber. The cut fins also cause large noise levels, but to a lesser extent than the long fins. The exception to this is the $761 \mathrm{~Hz}$ tone, which is almost $5 \mathrm{~dB}$ larger for the cut fins. This may be due to the cut fins being blunt providing a larger impingement surface. The impinging flow may be exiting the perforated cylinder perpendicular to its surface causing an impingement onto the walls of the outlet flow chamber thus exciting the natural acoustic modes. The poor acoustic performance of these configurations is undesirable so fin cones are not explored further.

\subsection{Closed Bottom Cone}

The axisymmetric silencer causes flow to be circumferentially exhausted with no directional bias, however, only one of the 4 walls of the outlet flow chamber is open. This means a part of the flow must be turned $180^{\circ}$ before exiting the outlet flow chamber. This may cause recirculation within the outlet chamber and lead to the excitation of natural modes. In an attempt to force the air to flow in a preferred direction, a special under-cone accessory is 3D printed. This accessory is essentially a projection of the cone to the perforated cylinder, shown in figure 8.8. This device should cause a bias to the airflow, making much less air flow in the non-desirable direction.

This accessory has an entirely negligible impact on measurement location B and the acoustic impact at location $\mathrm{F}$ is shown in figure 8.9 .

The noise generated seems to generally be louder, especially at higher frequencies. The peaks due to outlet chamber resonance at 900 and $1800 \mathrm{~Hz}$ are ever-present as 

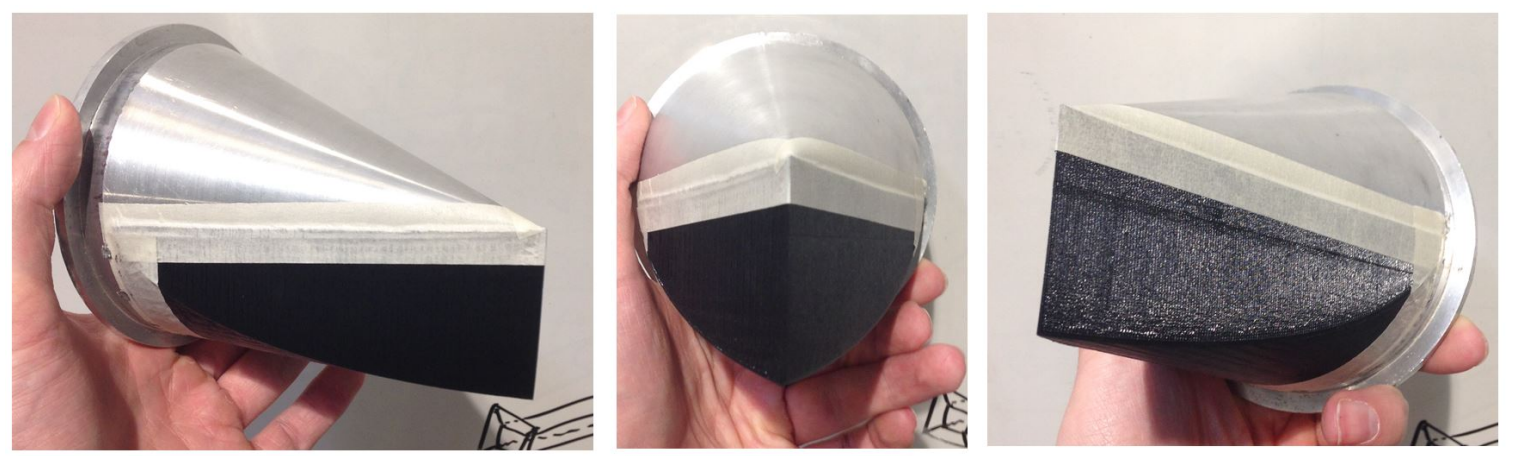

Figure 8.8: The closed bottom cone

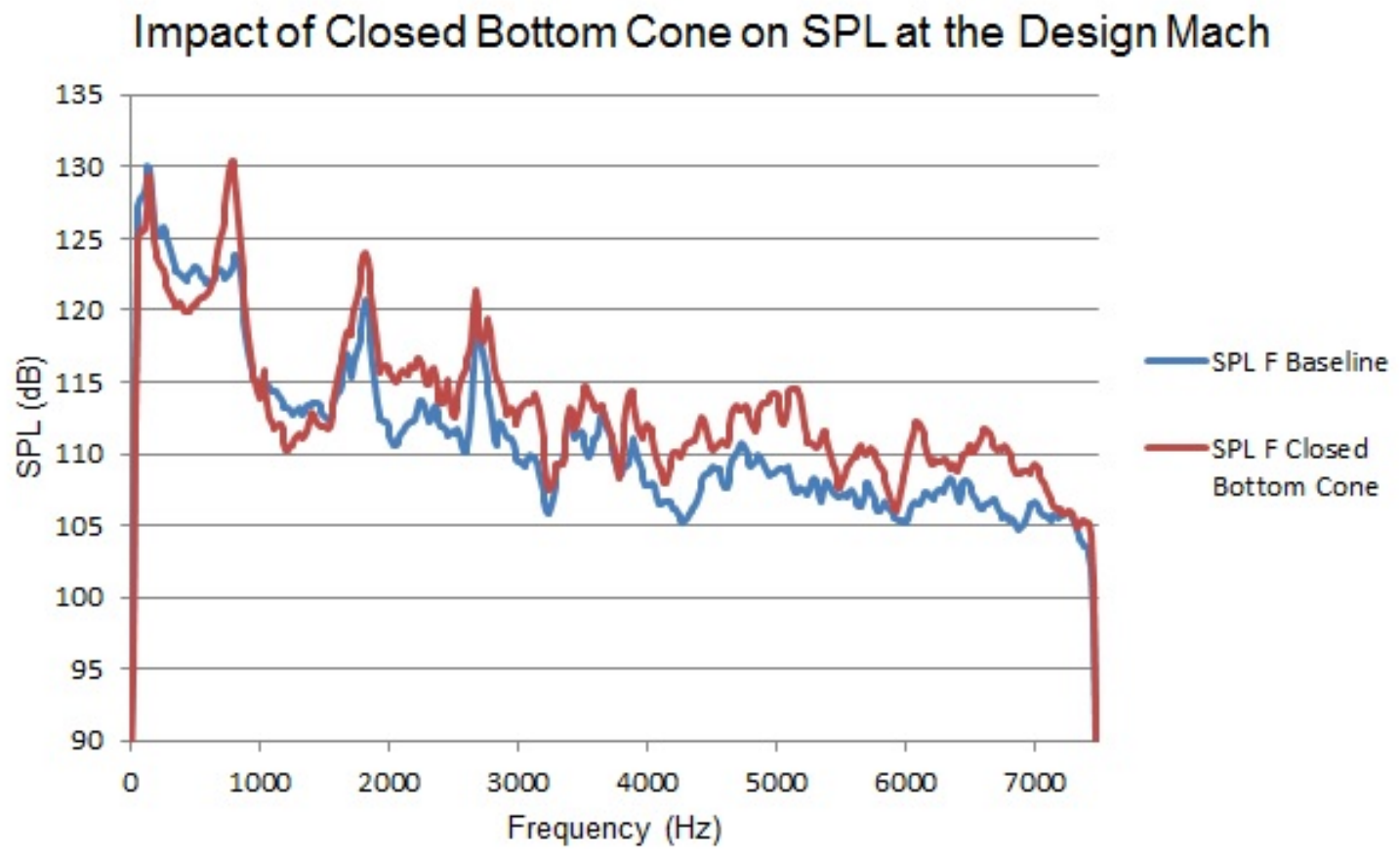

Figure 8.9: The SPL spectrum as measured at location $\mathrm{F}$ for the closed bottom cone

is the peak relating to throat resonance at $2600 \mathrm{~Hz}$. The peak generated at $761 \mathrm{~Hz}$ by the fin cones is also found here, centered at $776 \mathrm{~Hz}$ and of large magnitude. This supports the idea that a flow exiting the perforated cylinder in a more perpendicular manner may excite natural acoustic modes. 


\subsection{Elliptical Cone}

In a similar manner to the closed bottom cone, an elliptical cone is used in an attempt to improve the flow through the perforated cylinder. This shape of cone is obtained through revolution of half of an ellipse through its centerline, leading to a blunt cone tip with a tangential base. The surface profile is given by:

$$
y(x)=R \sqrt{1-\frac{x^{2}}{L_{\text {cone }}^{2}}}
$$

Where $y$ is the distance from the axis of rotation to the surface to the cone, $R$ is the radius at the base of the cone, $L$ is the length of the cone and $x$ is the distance along the centerline, ranging from 0 to $L$. This cone is used to attempt to balance the flow through the perforated cylinder, that is to increase the flow through the upstream portion and decrease the flow through the downstream portion. The 3D printed cone is shown in figure 8.10

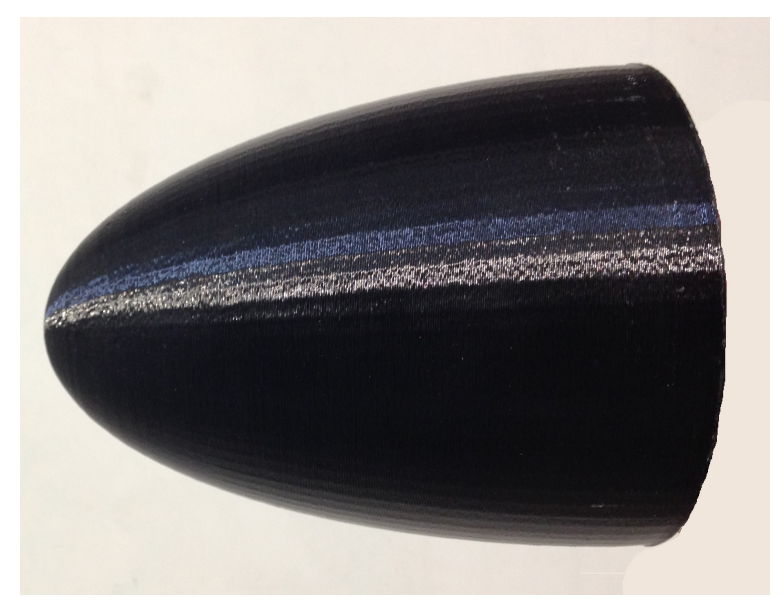

Figure 8.10: The elliptical cone

The impact of this cone on measurement location B is negligible other than for the first two frequency peaks which are reduced by 9 and $5 \mathrm{~dB}$ respectively, and the third peak which increases by $3 \mathrm{~dB}$. These peaks correspond to the entire ejector 
longitudinal mode and the first two longitudinal modes of the throat respectively. The reason for this change is unknown. The impact at measurement location $\mathrm{F}$ is greater and is shown in figure 8.11

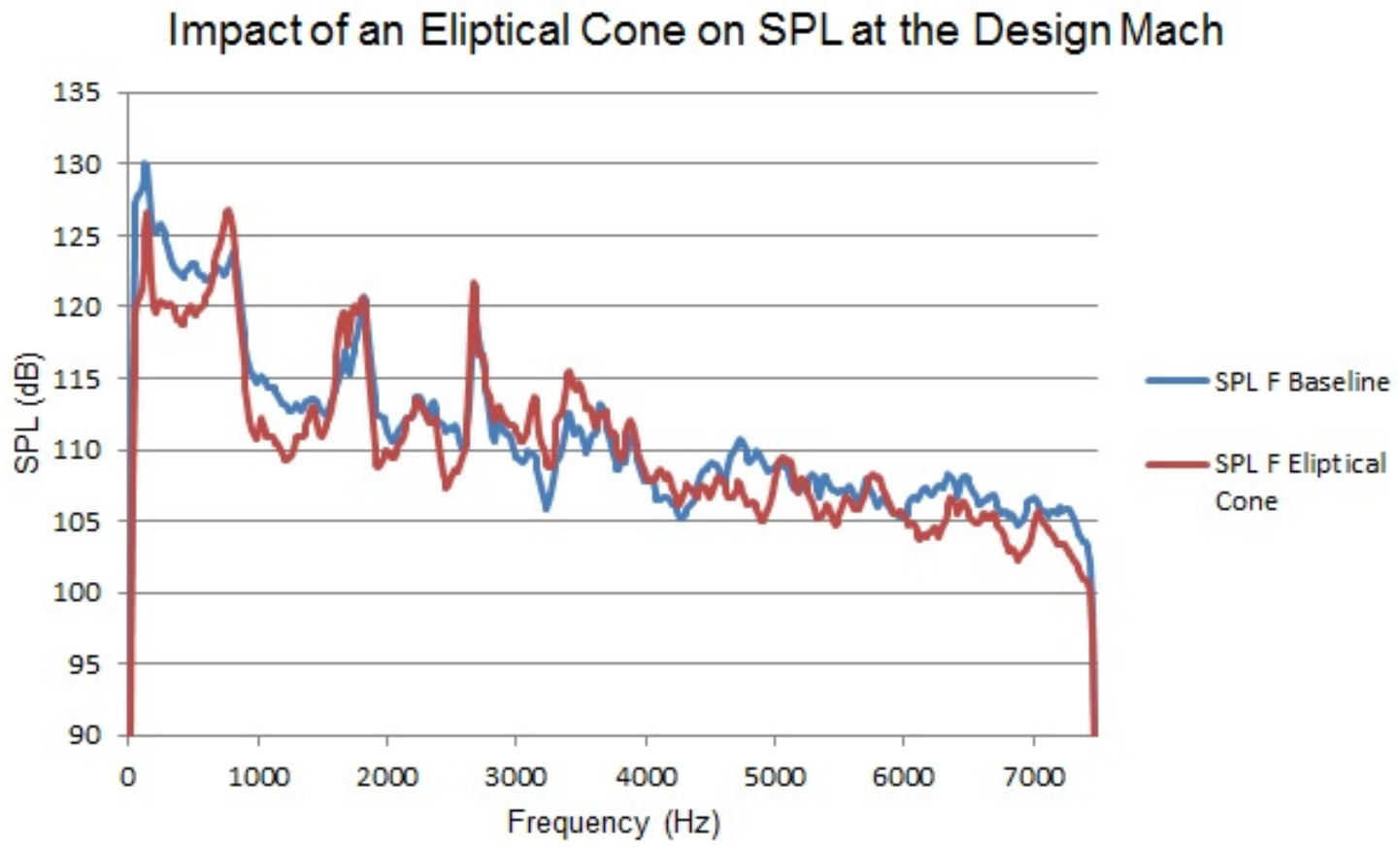

Figure 8.11: The SPL spectrum as measured at location F for the elliptical cone

The 0-600 Hz range is found to be of lesser amplitude, however, at $776 \mathrm{~Hz}$ the SPL magnitude increases, the same as for the fin and closed bottom cones. The outlet chamber resonance and throat resonance peaks at approximately 1800 and $2800 \mathrm{~Hz}$ remain unchanged. The entrainment ratio for this configuration is found to drop from 1.484 to 1.272 . While this is not negligible, it is not expected to have a dramatic effect on the acoustics. Overall the performance is marginally better than the baseline but these gains do not justify the increased manufacturing difficulties at the full scale. 


\subsection{Perforated Cones}

Three perforated cones are manufactured from 33\%, 40\% and 58\% OAR steel sheet. These OARs are chosen because any smaller causes too great of a pressure drop and any larger becomes too structurally weak. The cones are entirely perforated and have the same dimensions as the baseline cone. These cones can be seen in figure 8.12 and are used for variety of tests, each described in the following subsections.

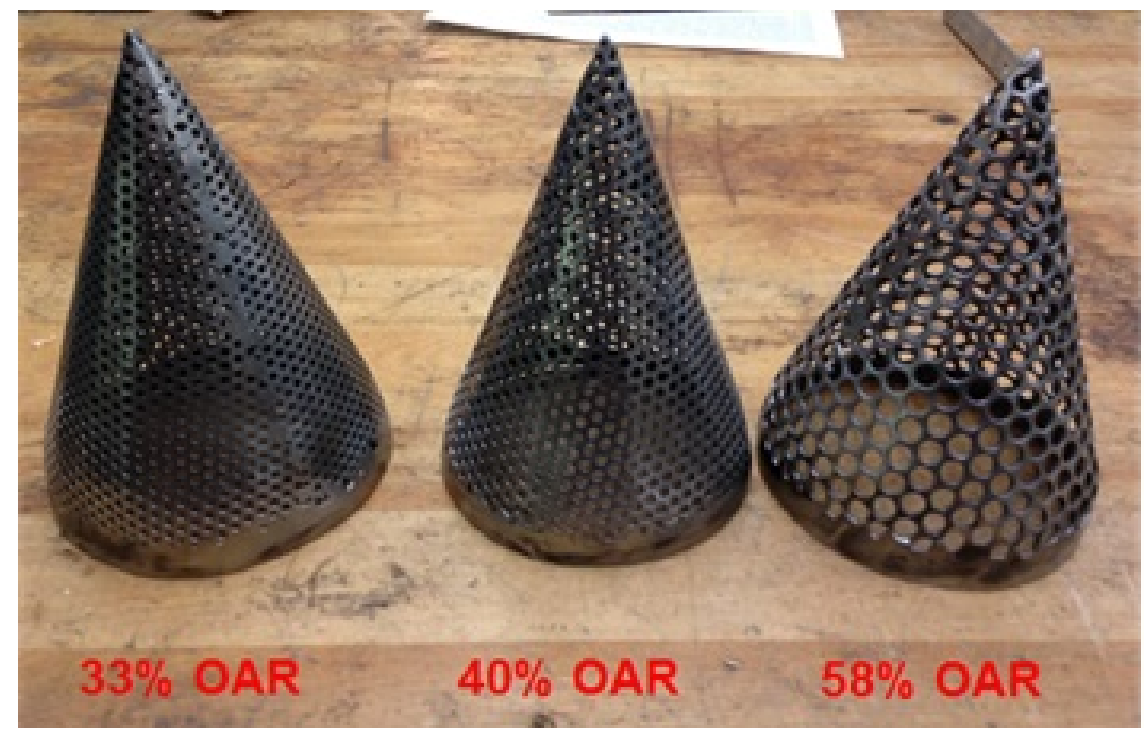

Figure 8.12: The perforated cones, used for multiple tests

\subsubsection{Perforated Cone Replacing The Solid Cone}

In reference [44], the use of a partially perforated cone can be seen. A perforated cone placed at the rear of the perforated cylinder would allow a portion of the flow to pass through the cone, decreasing the mass flow (thus velocity) of air flowing through the perforated cylinder.

The $58 \%$ OAR cone is omitted from these tests due to time constraints. Replacing the solid cone with either the $33 \%$ or $40 \%$ OAR cone is found to have a negligible 
impact at measurement location $\mathrm{B}$. The SPL plot at location $\mathrm{F}$ is shown in figure 8.13.

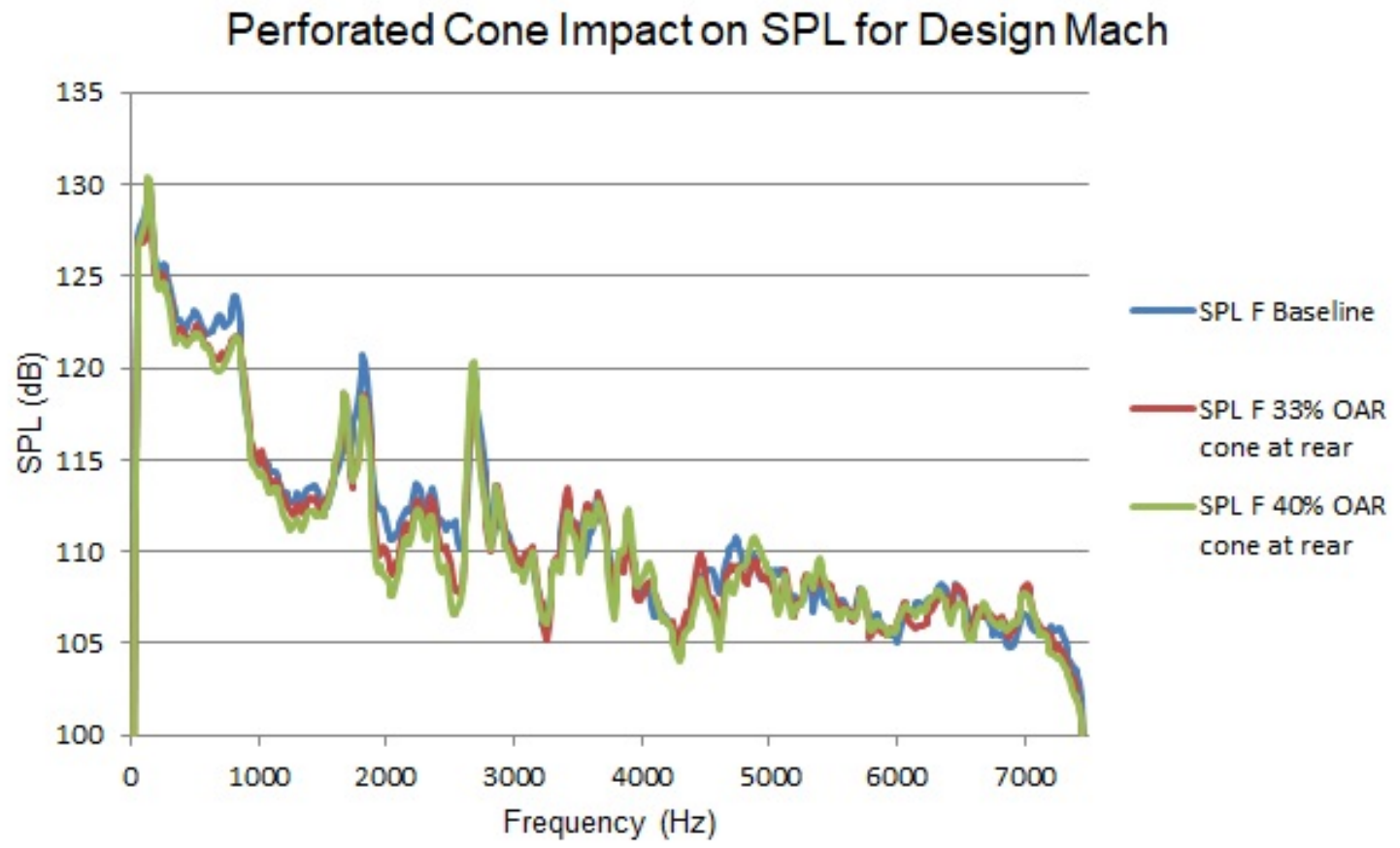

Figure 8.13: The SPL spectrum as measured at location $\mathrm{F}$ for the perforated cones

The spectrums are fairly similar to the baseline, with the tone at approximately $775 \mathrm{~Hz}$ of decreased magnitude. The outlet chamber resonant peak at approximately $1800 \mathrm{~Hz}$ also has a decreased magnitude. This may be due to the flow exiting the perforated cylinder to be of lower velocity due to a portion flowing through the cone. The valleys between 1000 and $2500 \mathrm{~Hz}$ show a decrease in magnitude, similar to the roughest grit cones. This may be due to the perforations acting somewhat like a roughness. The change from a solid to a perforated cone has a negligible impact on the entrainment ratio, estimated at 1.486 and 1.477 for the $33 \%$ and $40 \%$ OARs respectively, compared to the baseline 1.484. Given the marginal improvement in acoustics, the increased difficulty in manufacturing a perforated cone at the full scale 
is not deemed worthwhile.

\subsubsection{Perforated Cone In Front of Solid Cone}

The high velocity jet issuing from the primary nozzle travels downstream impinging on the cone, and impingement noise is sensitive to velocity. An attempt to slow the velocity before impact with the cone is made using the perforated cones. All three perforated cones are tested in conjunction with the solid cone. The chosen perforated cone is placed facing upstream in one of two locations: halfway between the diffuser outlet and cone base - referred to as "perforated cylinder midlength" - and at the diffuser end of the perforated cylinder - referred to as "in the diffuser".

\section{Perforated Cylinder Midlength}

Figure 8.14 shows the $58 \%$ OAR cone placed at the perforated cylinder midlength. It is believed that a cone at the midlength causes a portion of the incoming air to follow the profile of the perforated cone and exhaust through the perforated cylinder. The remaining air passes through the perforated cone before travelling a short distance downstream and impinging on the solid cone. This air will be of less velocity and mass flow than for the baseline configuration, hopefully decreasing the noise generation.

Surprisingly, the noise spectrum at location B remains nearly unchanged for all 3 perforated cones, however, the first two peaks are again affected. The first peak is decreased from 134 to $122 \mathrm{~dB}$ for all three cones. This is a massive difference. The second peak is decreased from 122 to 116,117 and $119 \mathrm{~dB}$ for the $33 \%, 40 \%$ and $58 \%$ perforated cones respectively.

In order to protect the microphones during testing, a few measurement locations

are omitted, including location F. For this reason the noise level at location $\mathrm{D}$ is 


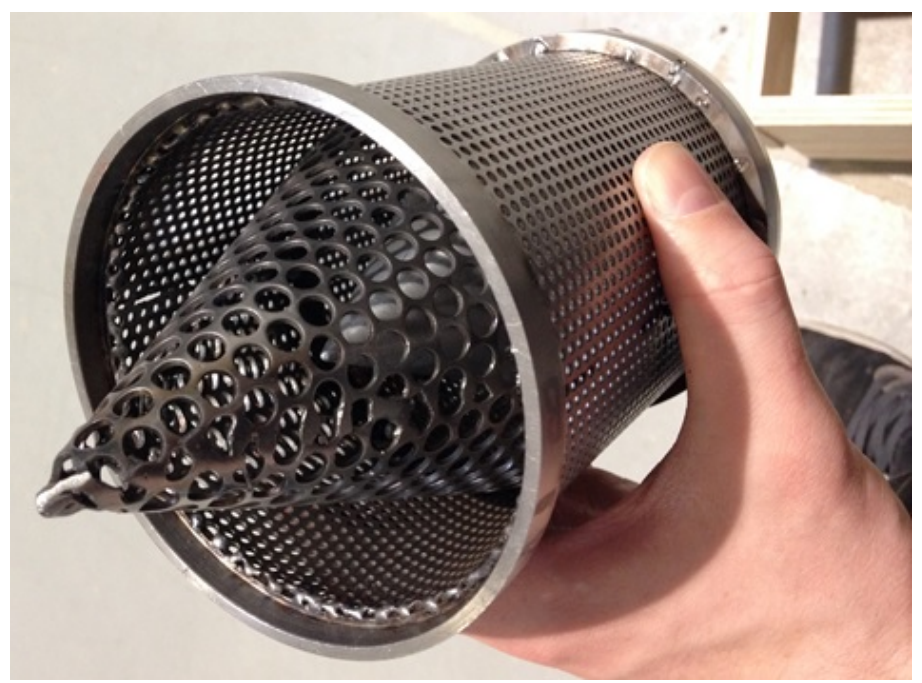

Figure 8.14: The 58\% OAR perforated cone placed at the perforated cylinder midlength with the solid cone visible behind it

presented in figure 8.15 .

\section{Impact of a Perforated Cone Placed at the Perforated Cylinder Midlength on SPL for the Design Mach}

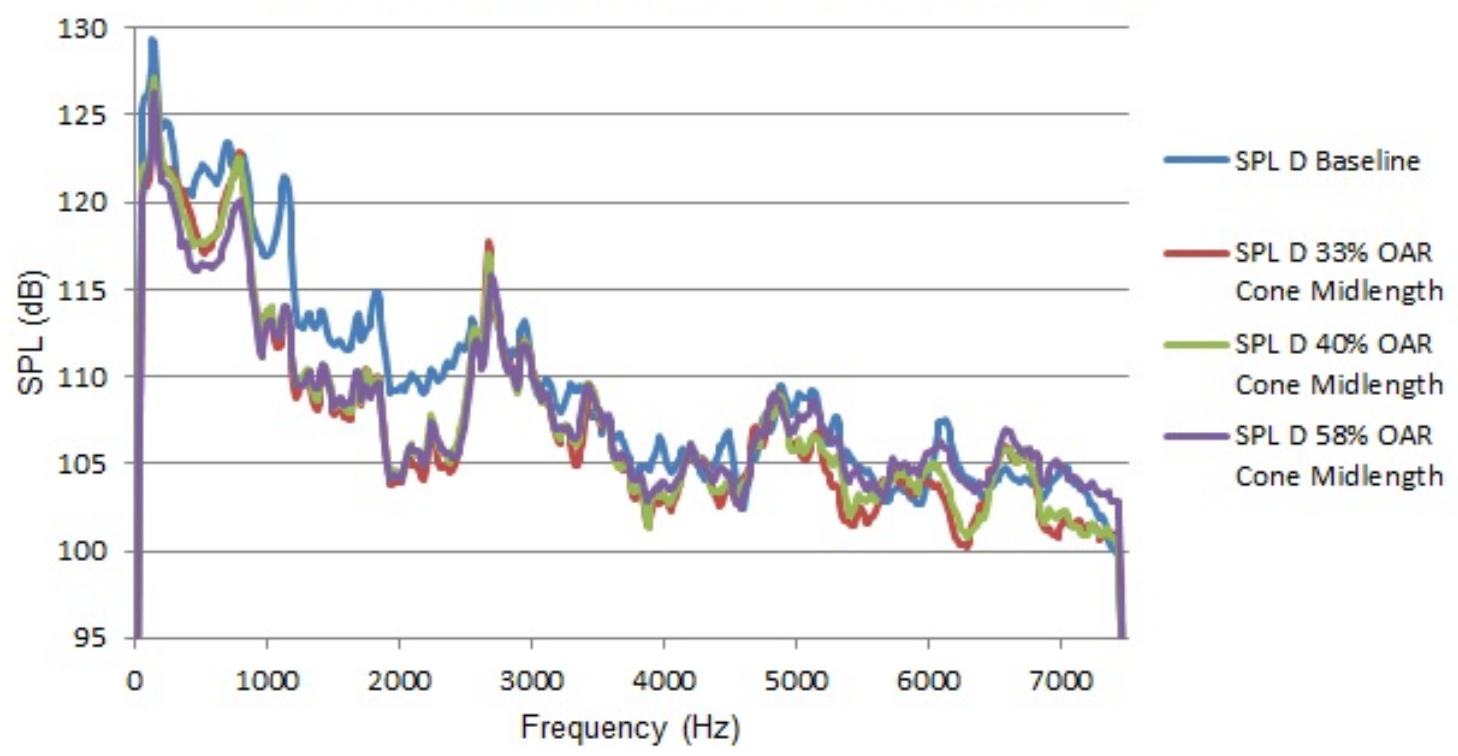

Figure 8.15: The SPL spectrum as measured at location D for the perforated cones placed at the midlength of the perforated cylinder 
The first peak is seen to decrease by 2 or $3 \mathrm{~dB}$ but the spectrum from this peak to approximately $2800 \mathrm{~Hz}$ is consistently lower than that of the baseline. This includes the secondary flow chamber and outlet chamber modes at approximately $1800 \mathrm{~Hz}$ which are decreased by $5 \mathrm{~dB}$. The peaks observed at $790 \mathrm{~Hz}$ for the fin cones, closed bottom cone and elliptical cones are present here but are below the baseline levels. This may suggest that the baseline configuration exhibits this large peak but that the high noise levels at surrounding frequencies cause it not to be as apparent. The perforated cones show a significant decrease at the neighbouring frequencies which may be the reason for this peak to appear to be so pronounced. The noise level above $3000 \mathrm{~Hz}$ is generally similar to that of the baseline configuration. An interesting observation is that below approximately $5000 \mathrm{~Hz}$ all three OAR's show very similar performance. This behaviour is not expected as it is believed that different OARs would provide drastically different flows. The reason for this similarity is unknown. The addition of a perforated cone inevitably increases the pressure loss and the entrainment ratio is estimated at 1.222 and 1.292 and 1.354 for the $33 \%, 40 \%$ and 58\% OARs respectively,

compared to the baseline 1.484. While this is lower, it is a tolerable drop given the acoustic performance increase, especially for the $58 \%$ OAR cone.

\section{In The Diffuser}

Unlike the perforated cylinder midlength configuration, the perforated cones placed in the diffuser do not allow any air to bypass the perforated cone and $100 \%$ of the flow must pass through the perforated cone. Figure 8.16 shows a photo of the installation along with a schematic of the ejector silencer in this configuration.

The $40 \%$ OAR cone is omitted from these tests due to time constraints. The acoustic spectrum at measurement location B is found to mostly remain the same, 

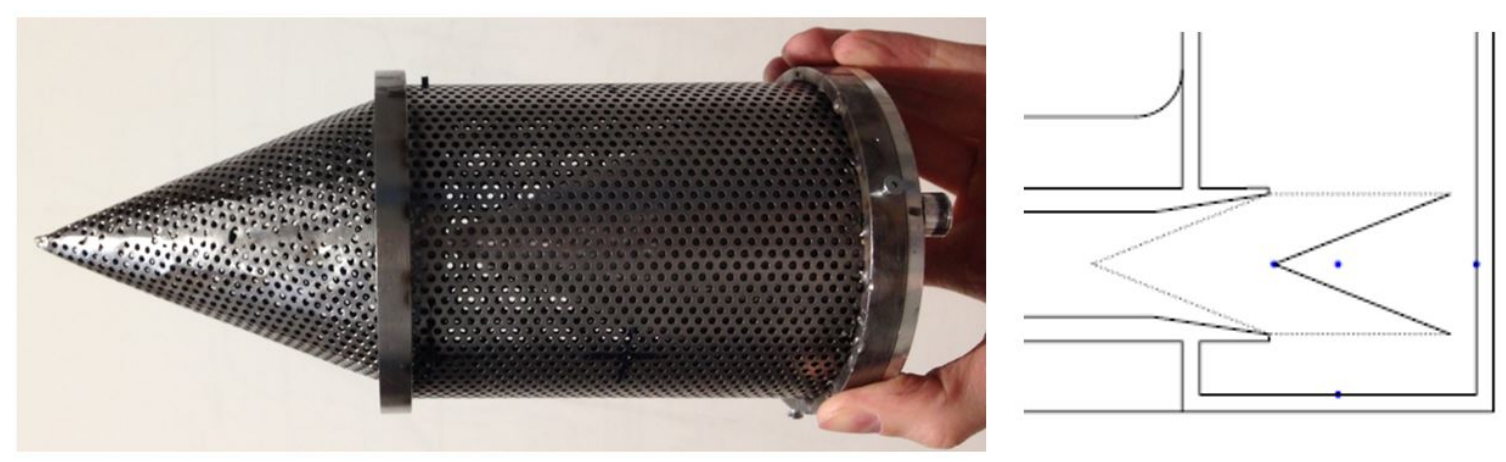

Figure 8.16: The $33 \%$ perforated cone placed "in the diffuser" (left) and a cross sectional schematic of this configuration (right)

however, the lowest frequencies are again affected. The whole ejector longitudinal tone is reduced by $10 \mathrm{~dB}$ and $14 \mathrm{~dB}$ for the $33 \%$ and $58 \%$ perforated cones respectively, which is an extremely significant amount. A slight performance increase is also apparent at a few other frequencies. The performance at measurement location $\mathrm{F}$ is drastically different than that of the baseline and it is shown in figure 8.17. 


\section{Impact of a Perforated Cone Placed in the Diffuser on the SPL for the Design Mach}

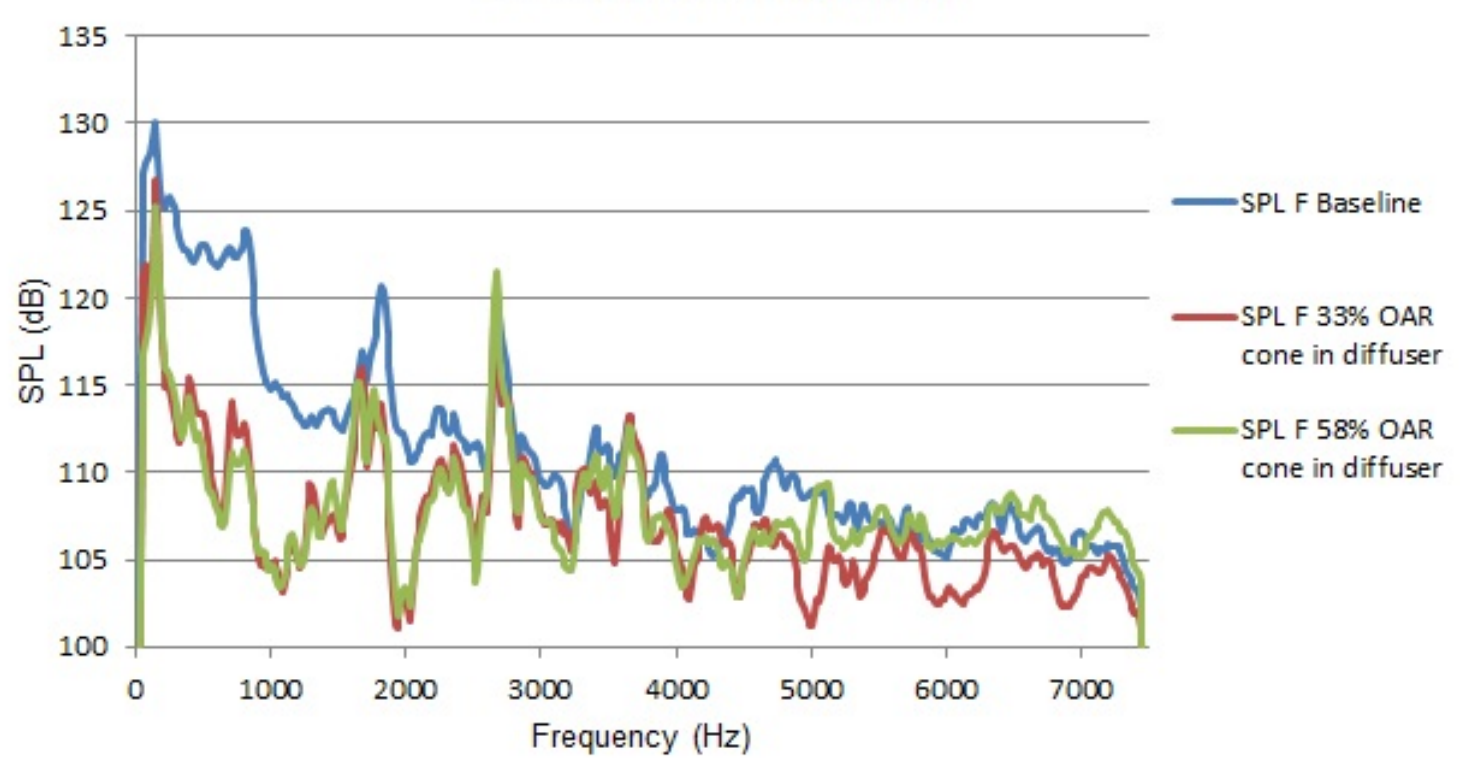

Figure 8.17: The SPL spectrum as measured at location F for the perforated cones placed in the diffuser

Although it is tempting to dive into the details of these configurations, the entrainment ratio is found to be drastically reduced. It is estimated at 0.535 and 1.054 for the $33 \%$ and $58 \%$ OARs respectively, compared to the baseline 1.484. This is a significant drop which inevitably impacts ejector performance and acoustics. This drop is deemed too large and no further investigation into these configurations is undertaken.

\subsubsection{Reverse Perforated Cone}

The Flowmaster(R)dBX@Series laminar flow automobile mufflers feature a design where the incoming flow is forced through a reverse perforated cone before passing through a forward facing perforated cone. This design inspired the testing of the perforated cones placed at the diffuser outlet in a reversed orientation. Two attempts 
at this are made and they are discussed in the following subsections.

\section{Within Perforated Cylinder}

Only the $33 \%$ OAR cone is tested in the reverse configuration. The test is performed with the cone "in the diffuser" but with the apex of the cone located downstream. The reverse perforated cone is placed inside the perforated cylinder leaving no room for the solid cone. For this reason, a special perforated disk is manufactured which is used to close the rear of the perforated cylinder. Figure 8.18 shows this configuration.

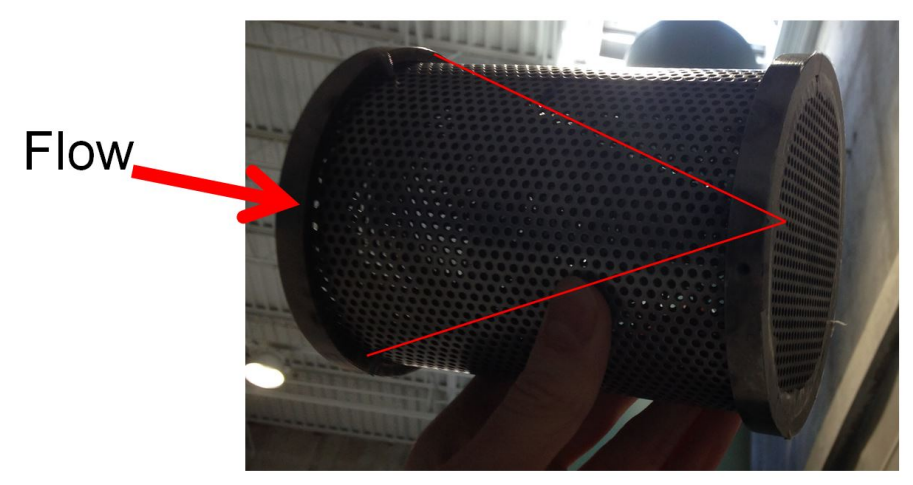

Figure 8.18: The reverse perforated cone within the perforated cylinder

The resulting sound pressure level at measurement location B is relatively unchanged but at location $\mathrm{F}$ the change is significant. The spectrum is shown in figure 8.19 .

The entire ejector longitudinal mode is found to be unaffected but with this peak excluded, the acoustic levels are found to be much lower. Interestingly, the magnitude of the tone at approximately $775 \mathrm{~Hz}$ is less, however, the relative magnitude of the tone at $1800 \mathrm{~Hz}$ is found to increase. This is an interesting behaviour, essentially reducing the oscillation amplitude of the first mode and increasing that of the second mode. The reason for this behaviour is unexplained. The throat modes are also 


\section{Impact of a Reverse Perforated Cone in the Diffuser on the} SPL for the Design Mach

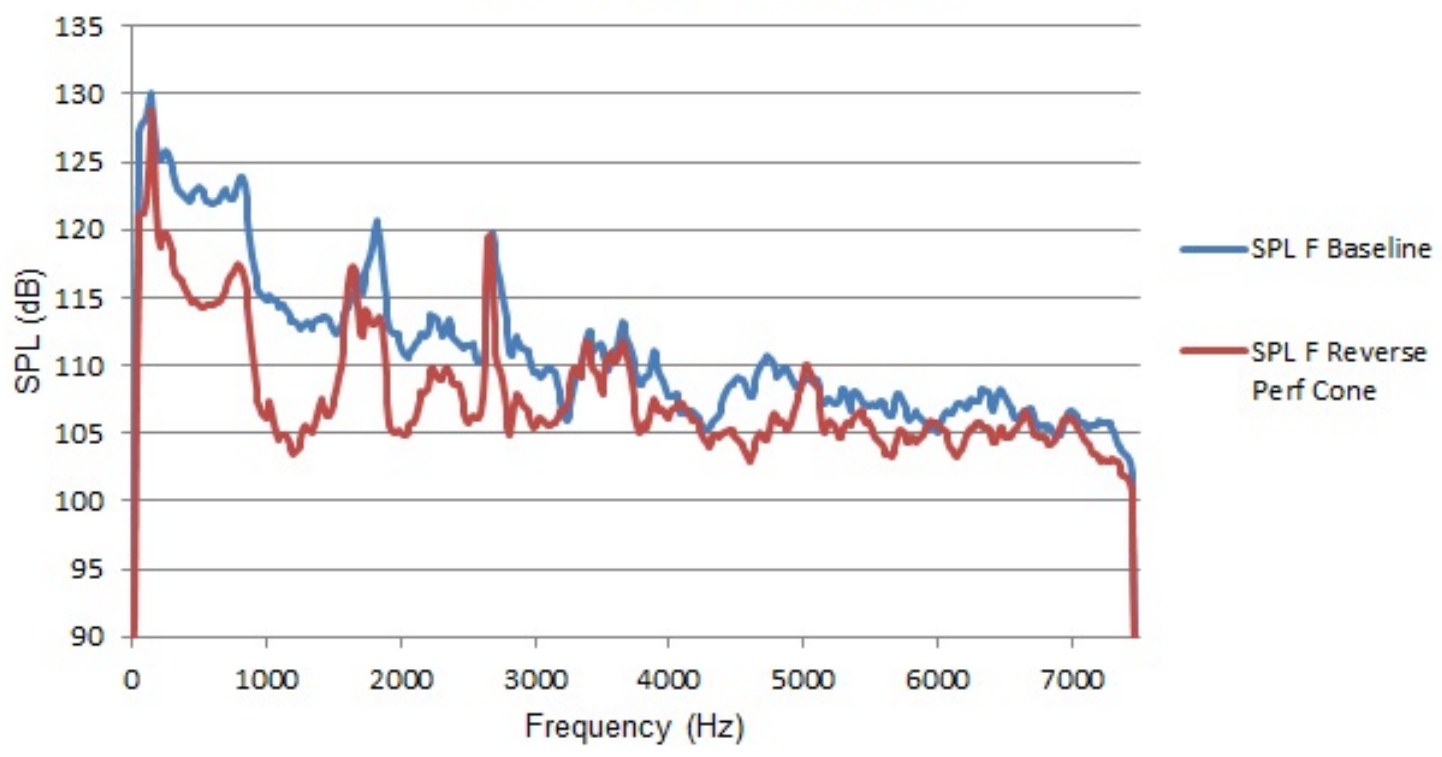

Figure 8.19: The SPL spectrum as measured at location $\mathrm{F}$ for the reverse perforated cone within the perforated cylinder

present and of a similar magnitude to the baseline but they appear as a much more defined peak. The entrainment ratio for this configuration is found to be significantly decreased to approximately 1.121 from the baseline 1.484 . This is a large drop and the acoustic improvement may be due to the increased pressure loss. It is difficult to draw any conclusions from these results without any knowledge of how this configuration changes the airflow.

\section{Instead of Perforated Cylinder}

Given the large decrease in entrainment ratio encountered with the reverse perforated cone tested within the perforated cylinder, a special reverse perforated cone is manufactured such that it can be tested without the perforated cylinder. This cone has a base diameter matching that of the diffuser outlet. The cone extends from the 
diffuser outlet to the base assembly making contact with the base wall. Figure 8.20 is a schematic of this configuration.

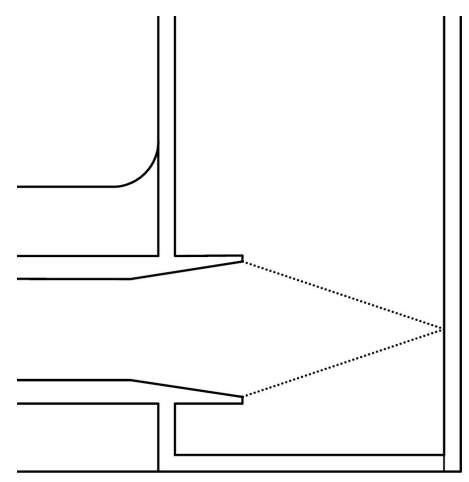

Figure 8.20: Schematic of the reverse perforated cone tested without the perforated cylinder

The acoustic impact at location B is again entirely negligible. The general trend at location $\mathrm{F}$, shown in figure 8.21, is found to be quite different to that from the reverse perforated cone within the perforated cylinder. The acoustic levels are found to be higher for nearly all frequencies, peaks and valleys alike. Interestingly, the second octaves of the room modes which are clumped together for the baseline at $1800 \mathrm{~Hz}$ appear to separate with this configuration. The small difference in second octaves in the $\mathrm{x}$ and $\mathrm{y}$ directions $(1788$ and $1675 \mathrm{~Hz}$ estimated, $\mathrm{y}$ in the direction of the throat) appear to manifest as two separate peaks at 1860 and $1684 \mathrm{~Hz}$ respectively. The baseline does show a small peak at $1684 \mathrm{~Hz}$. This may imply that the y direction mode is amplified with the new configuration. The baseline has a solid cone which prevents air from flowing in the y direction, however, this configuration allows a significant amount of air to flow in the y direction, possibly accounting for this different behaviour. Although the entrainment ratio only changes to 1.472 as compared with the baseline 1.484, this configuration negatively impacts the acoustics thus is not investigated further. 


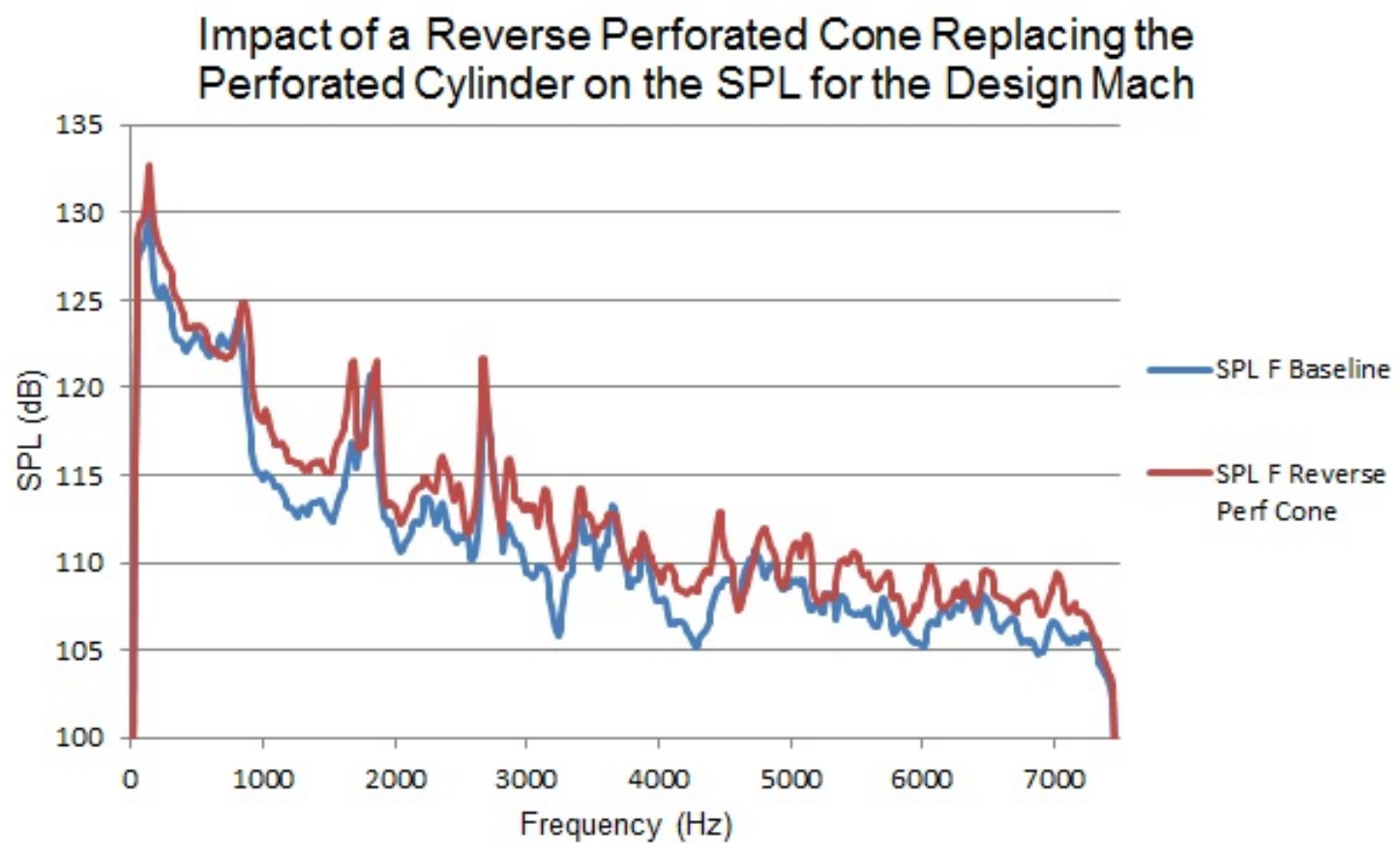

Figure 8.21: The SPL spectrum as measured at location $\mathrm{F}$ for the reverse perforated cone tested without the perforated cylinder

\section{7 $\quad$ Perforated Disks}

The perforated cones placed at the midlength provide a significant performance increase, however, manufacturing of such cones, even at the small scale, is difficult. For this reason the impact of placing a perforated disk - essentially a flat perforated sheet, similar to a cone with apex angle $180^{\circ}$ - in front of the cone is examined.

\subsubsection{3\% OAR and 58\% OAR}

The solid cone extends past the midlength of the perforated cylinder, therefore, the perforated disks cannot be used at this location. The $33 \%$ and $55 \%$ OAR perforated disks are placed at the "in the diffuser" location. The resulting entrainment ratios are estimated at 0.649 and 1.205 for the $33 \%$ and 55\% OAR disks respectively, compared to the baseline 1.484 (and compared to 0.535 and 1.054 for the $33 \%$ and $58 \%$ 
OAR perforated cones at the same location). This entrainment is too low and this configuration is unacceptable, however, some interesting observations can be made from the results, shown in figure 8.22 , which also includes the previous results for the perforated cones in the diffuser. 


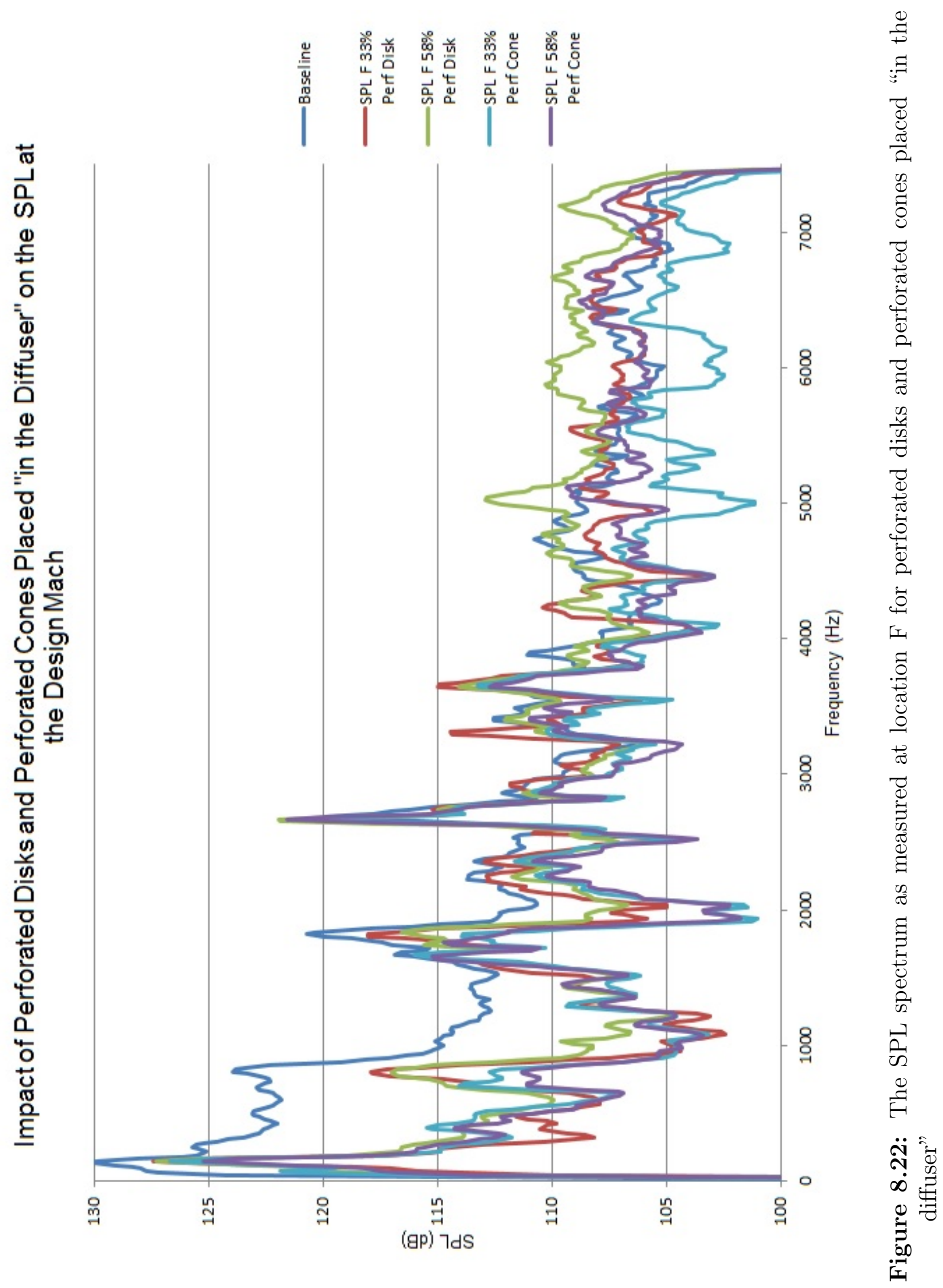


Somewhat surprisingly, the performance of the perforated disks is found to be quite similar to that achieved with the perforated cones. The entire ejector longitudinal mode is better attenuated by the cones, but the difference is only by $3 \mathrm{~dB}$ at most. Interestingly, the exhaust first acoustic natural modes at $775 \mathrm{~Hz}$ are of greater magnitude for the perforated disks (relative to the perforated cones). It is found that both plates are approximately $5 \mathrm{~dB}$ louder at this frequency than the perforated cones. The eddies shed from the disks may be more uniform and organized leading to this greater excitation. The remaining frequencies from 1000 - $4000 \mathrm{~Hz}$ show similarity and trends/relations are hard to establish at higher frequencies.

\subsubsection{Perforated Disks}

Rather than diverting the flow with a cone, an attempt at slowing the flow using 3 perforated disks in the perforated cylinder (without a cone) is made. The disks are placed in decreasing OAR, that is the $58 \%$ OAR disk is furthest upstream at the "in the diffuser" location, the $40 \%$ OAR disk is placed at the midlength and the $33 \%$ OAR disk is located where the base of the solid cone would normally be. The acoustic results for this configuration are shown in figure 8.23. Measurement location $\mathrm{B}$ is found to have the first 3 peaks reduced by 10,8 and $3 \mathrm{~dB}$ respectively with little other differences compared to the baseline.

The entrainment ratio for this configuration drops substantially, from the baseline 1.484 to 1.092. Given this large drop, similar in magnitude to 55\% OAR perf cone placed in the diffuser, it would be expected that the acoustic performance would be better improved. In this configuration the flow has a greater surface through which it can be released into the outlet flow chamber and this spreading of the flow may be the source of the decreased noise at the first natural acoustic mode of the 


\section{Impact of Replacing the Cone with 3 Perfoated Plates on the SPL at the Design Mach}

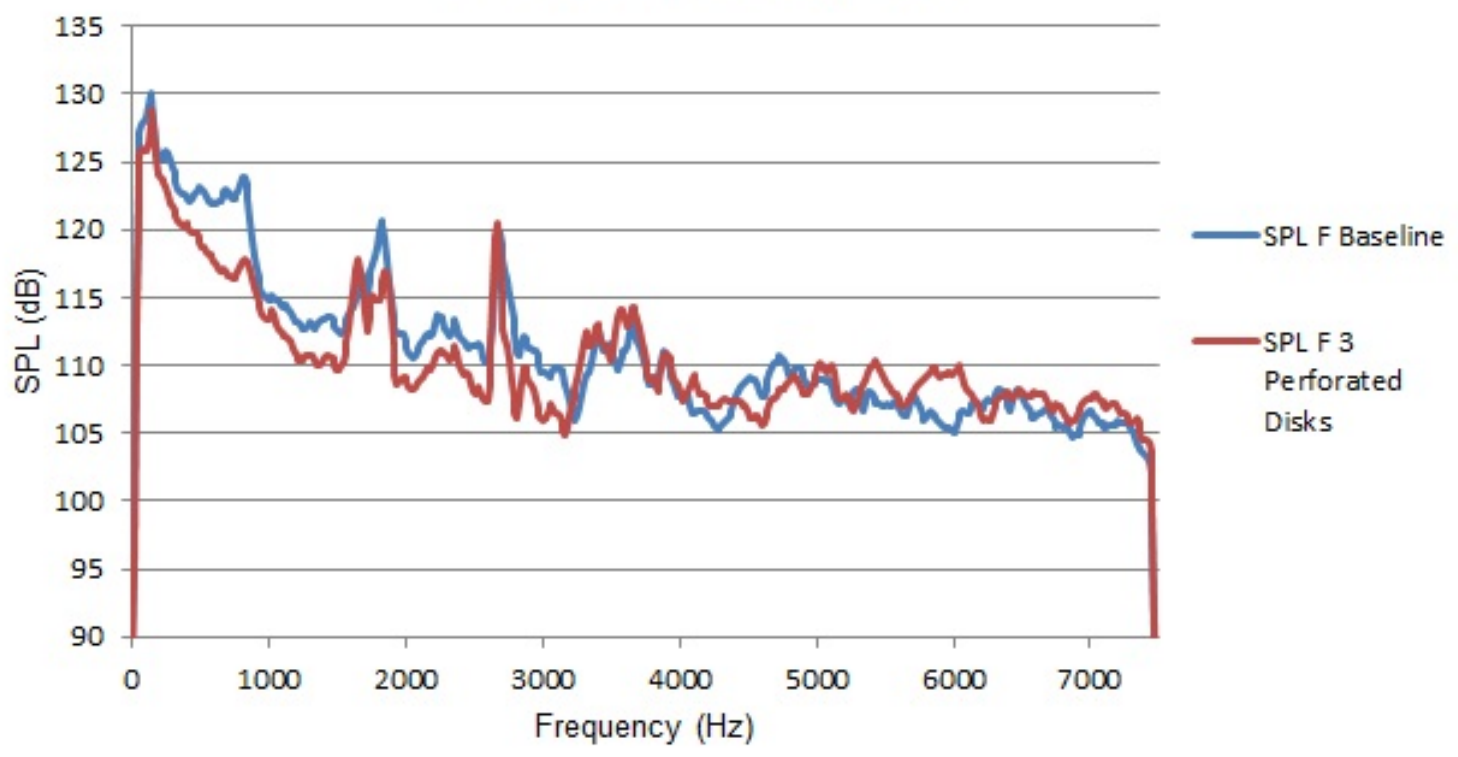

Figure 8.23: The SPL spectrum as measured at location $\mathrm{F}$ for 3 perforated disks replacing the baseline cone

outlet flow chamber at $775 \mathrm{~Hz}$. A slight reduction is observed from the first peak to approximately $1800 \mathrm{~Hz}$, where the second octave of the natural modes of the outlet flow chamber cause a large peak. This configuration again allows a flow in the $y$ direction and the second octaves of the outlet flow chamber at $1800 \mathrm{~Hz}$ show two distinct peaks rather than 1 . The natural modes of the throat also cause their regular peak at approximately $2600 \mathrm{~Hz}$. The drop in entrainment ratio is too large and the acoustic improvement too small for this configuration to merit further consideration.

\subsection{Diffuser Modifications}

On a completely different investigational path, some configuration changes are made to the diffuser to observe their impact. This idea stems from the work of reference [69] where the boundary conditions at the diffuser inlet are found to be important to 
tone generation as well as the work of reference [32] where the placement of a screen at the aft of a pipe reduces tones significantly.

\subsubsection{Screen at the Diffuser Inlet}

In an attempt to modify the boundary conditions at the diffuser inlet, a screen is installed. This screen is leftover from the primary nozzle flow conditioning screen, which has a $65 \%$ OAR with a wire diameter of 0.01 inches $(0.2559 \mathrm{~mm})$ and is 30 wire mesh. Initially, the screen was placed between the diffuser sleeve and throat and held in place using only friction. This led to the screen being blown out of place. The screen was then taped to the diffuser sleeve and the test repeated. The screen was ripped causing a large hole, as can be seen in figure 8.24 .

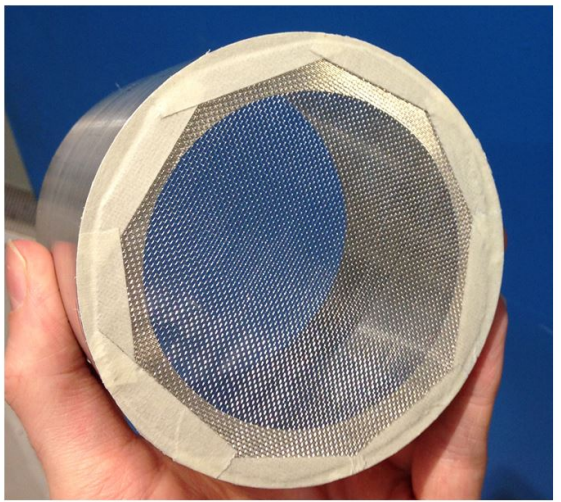

Before

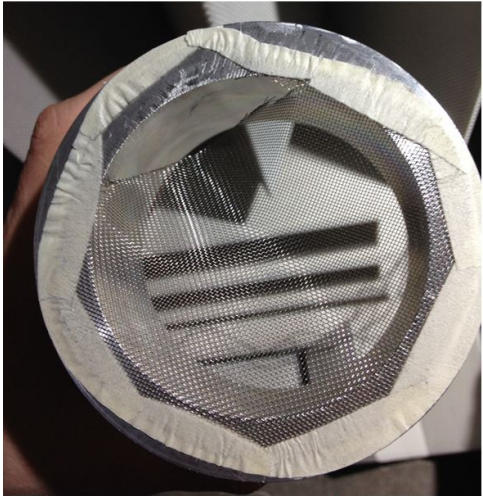

After

Figure 8.24: The screen displaced from the diffuser inlet

A new screen was manufactured, similar to that used by [32], where the periphery of the screen contains holes. This screen was destroyed during testing, with the portion of screen between the holes ripping. Finally, in an attempt to avoid screen failure, an aluminum plate with a series of peripheral holes $\rrbracket^{1}$ was secured between

\footnotetext{
${ }^{1}$ This plate was not engineered in any manner, but rather was quickly manufactured and tested due to time constraints
} 
the diffuser sleeve and throat. This plate also failed, with the solid central portion breaking free. Despite these failures, the acoustic spectrum for these configurations as measured at location $\mathrm{F}$ is shown in figure 8.25 .

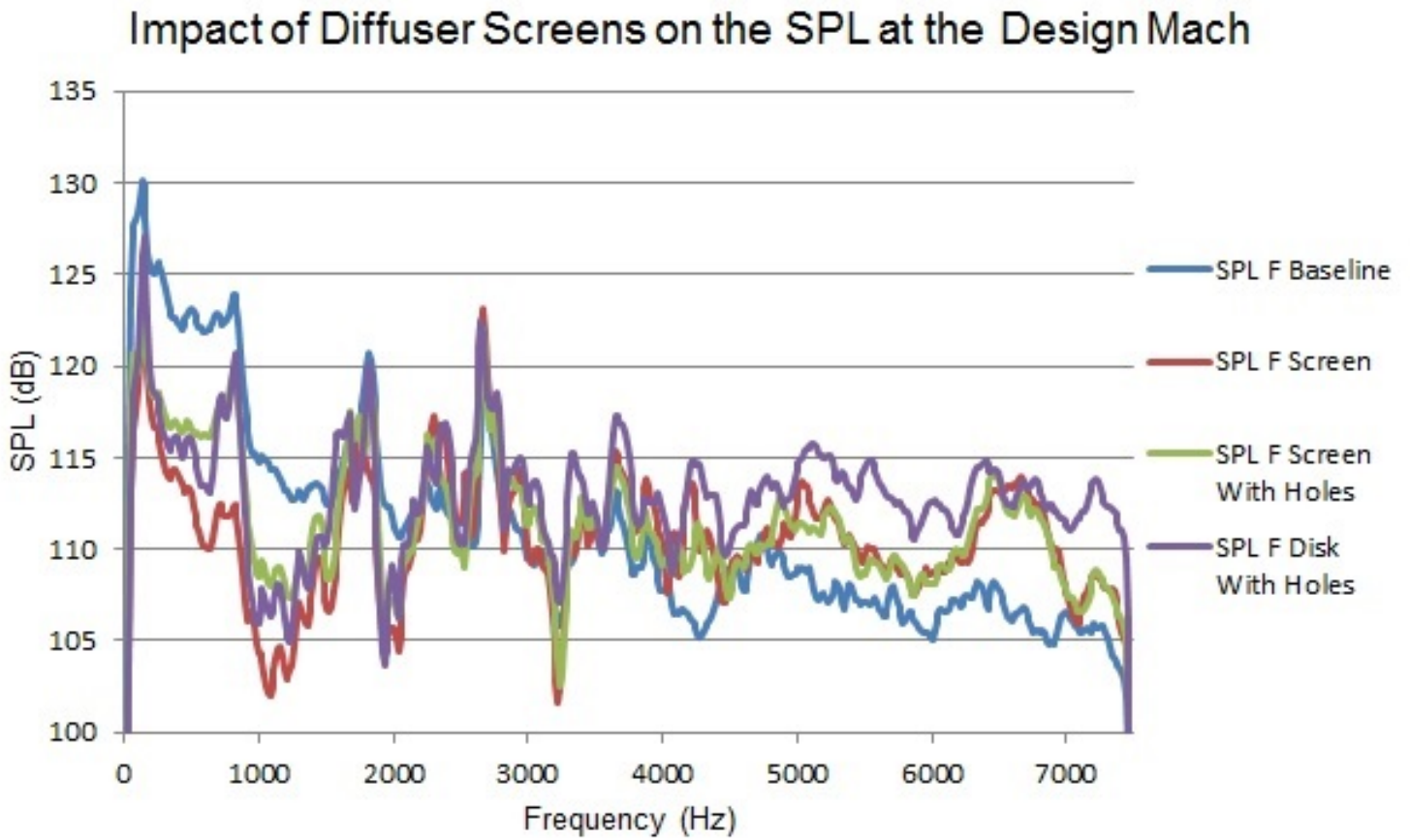

Figure 8.25: The SPL spectrum as measured at location F for the 3 types of screens placed at the diffuser inlet, all of which were damaged

The author is weary of drawing conclusions from any of this data as it is unknown what the condition of the screen/plate was at the time of acquisition. General trends which are seen are that the screens tend to decrease the SPLs below $1800 \mathrm{~Hz}$, have no impact on the second modes of the outlet flow chamber or fundamentals of the throat and generate greater noise levels at higher frequencies. The generation of greater noise levels at higher frequencies is not unexpected as the screens do shed small eddies. Noise at higher frequencies tends to be easier to attenuate thus this shifting of low frequency noise to higher frequencies could be beneficial. Interestingly, the tone at $775 \mathrm{~Hz}$ is more prominent for the screen with holes and the disk with 
holes. This is perplexing but may be due to the condition of the devices at the time of recording. The entrainment ratios for the three configurations are found to drop from the baseline 1.484 to 1.124 for the regular screen, 1.261 for the screen with peripheral holes and to 0.503 for the aluminum plate with peripheral holes. From these tests, it can be concluded that the force generated by the primary jet is quite large, even at this small scale. The use of such a device on the full scale would be challenging and likely require continuous maintenance.

\subsubsection{No Diffuser Sleeve}

Simply out of curiosity, the ejector is tested without the diffuser sleeve in place. This causes a step increase in diameter near the outlet of the ejector which is expected to be a significant source of recirculation and noise (this geometry is similar to that of a whistler nozzle, see reference [110]). The resulting spectrum as measured at location $\mathrm{F}$ is given in figure 8.26

Across all frequencies the SPL is found to be greater. The outlet flow chamber modes at 900 and $1800 \mathrm{~Hz}$ are amplified, which is not unexpected, and the transverse/azimuthal throat modes appear unchanged both in magnitude and frequency. The entrainment ratio is found to decrease slightly from 1.484 to 1.461 . This is likely due to an increased pressure loss from the recirculation occurring in the diffuser.

\subsection{Natural Mode Elimination}

From all previous tests, it appears as though the natural modes of the ejector are significant contributors to the overall noise levels and occur regardless of the silencer configuration. The following two subsections describe tests undertaken in an attempt 


\section{Impact of Removing the Diffuser Sleeve (step diameter increase) on the SPL at the Design Mach}

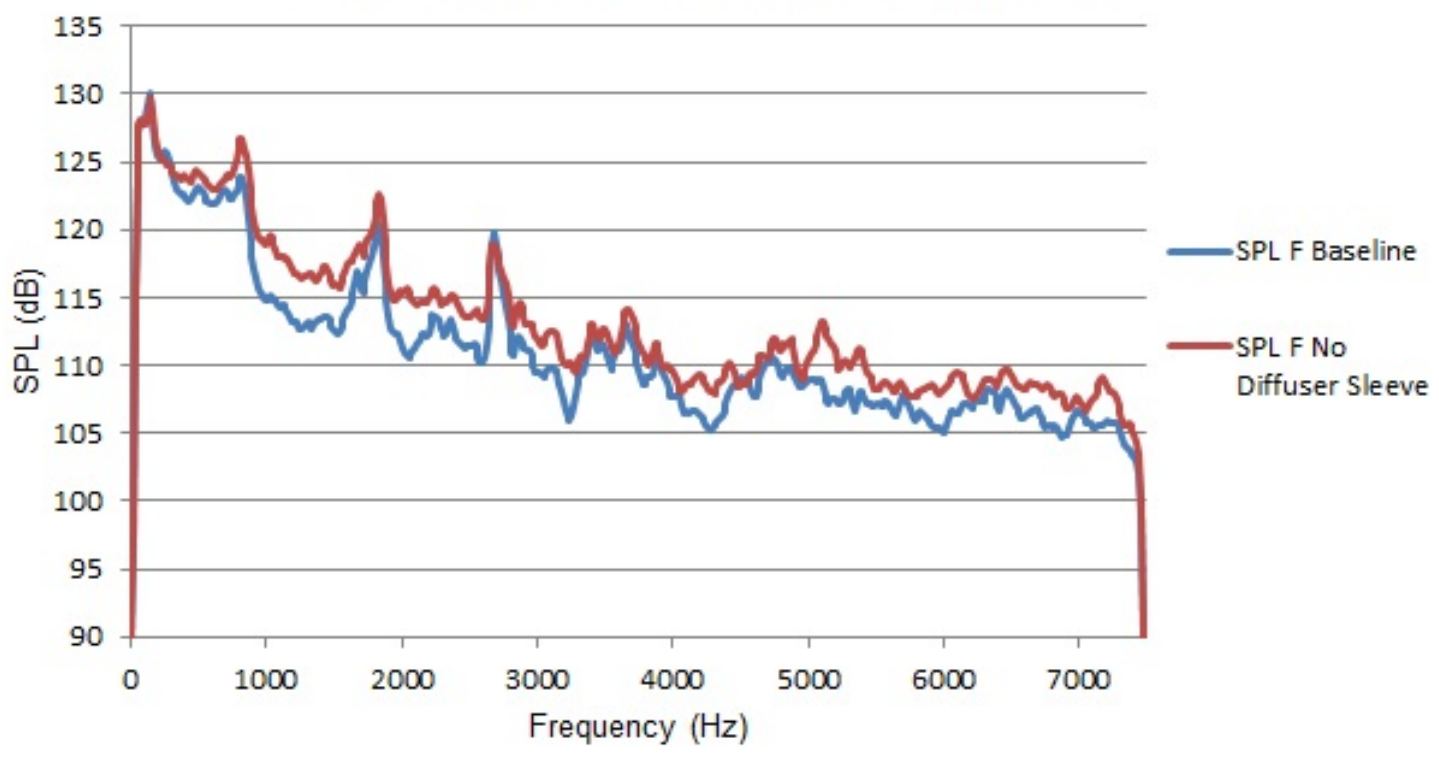

Figure 8.26: The SPL spectrum as measured at location F for the ejector without a diffuser sleeve

to alter the behaviour of these natural modes.

\subsubsection{Outlet Flow Chamber Rounded Corners}

In an attempt to reduce the level of excitation of the outlet flow chamber natural modes, rounded base corners made of styrofoam are manufactured and installed, as seen in figure 8.27. These corners are used in an attempt to reduce flow recirculation in the outlet flow chamber and help direct the flow towards the outlet.

Surprisingly, it is found that these rounded corners have an essentially negligible impact on the acoustics at location $\mathrm{F}$, as shown in figure 8.28. It would seem that the natural modes are likely excited by a mechanism other than recirculation, possibly the broadband noise of the flow itself or the jet exiting the perforated cylinder interacting with the outlet flow collector walls. The natural acoustic modes of the outlet flow 


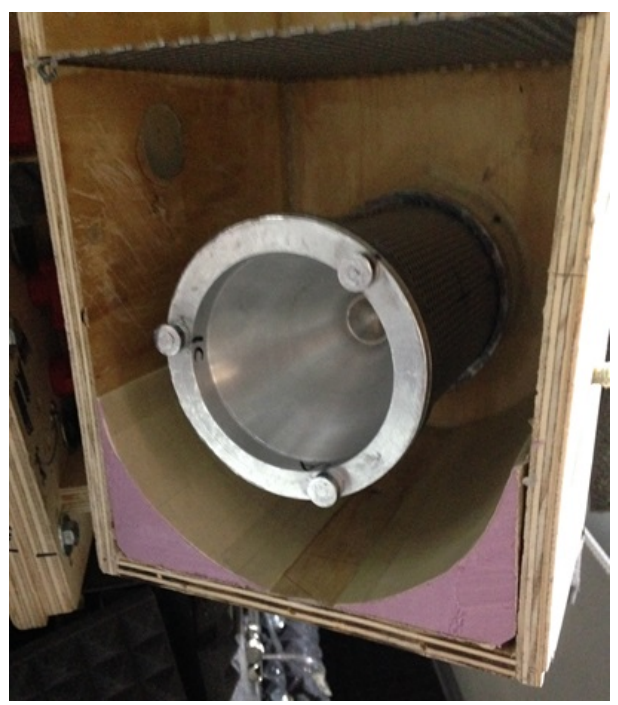

Figure 8.27: The rounded corners employed in the outlet flow chamber

chamber appear essentially unchanged. The entrainment ratio for this configuration surprisingly drops from the baseline 1.484 to 1.364 . This implies that the pressure loss from the rounded corners is greater than that of the non-rounded corners. It may be that recirculation bubbles which form in the baseline configuration take on an optimal shape and act as the ideal flow diverters ${ }^{2}$. As a side note, a drop in entrainment ratio from 1.484 to 1.364 is seen to have a negligible impact on the acoustics.

\subsubsection{Scale Model Lined With Acoustic Foam}

A final test is attempted where the outlet flow chamber walls are lined with acoustic foam. This acoustic foam changes the ability of the walls to reflect sound waves and in theory should significantly reduce or eliminate the natural modes. The secondary flow chamber is also lined with foam, however, there is not enough foam available to cover the entire secondary flow chamber thus only about half of it is lined. Figure 8.29 shows a picture of the outlet flow chamber lined with foam and the new microphone

\footnotetext{
${ }^{2}$ This is the case for a step diffuser, where the recirculation bubble formed provides a very good diffusion angle for the given flow, see reference 23 .
} 


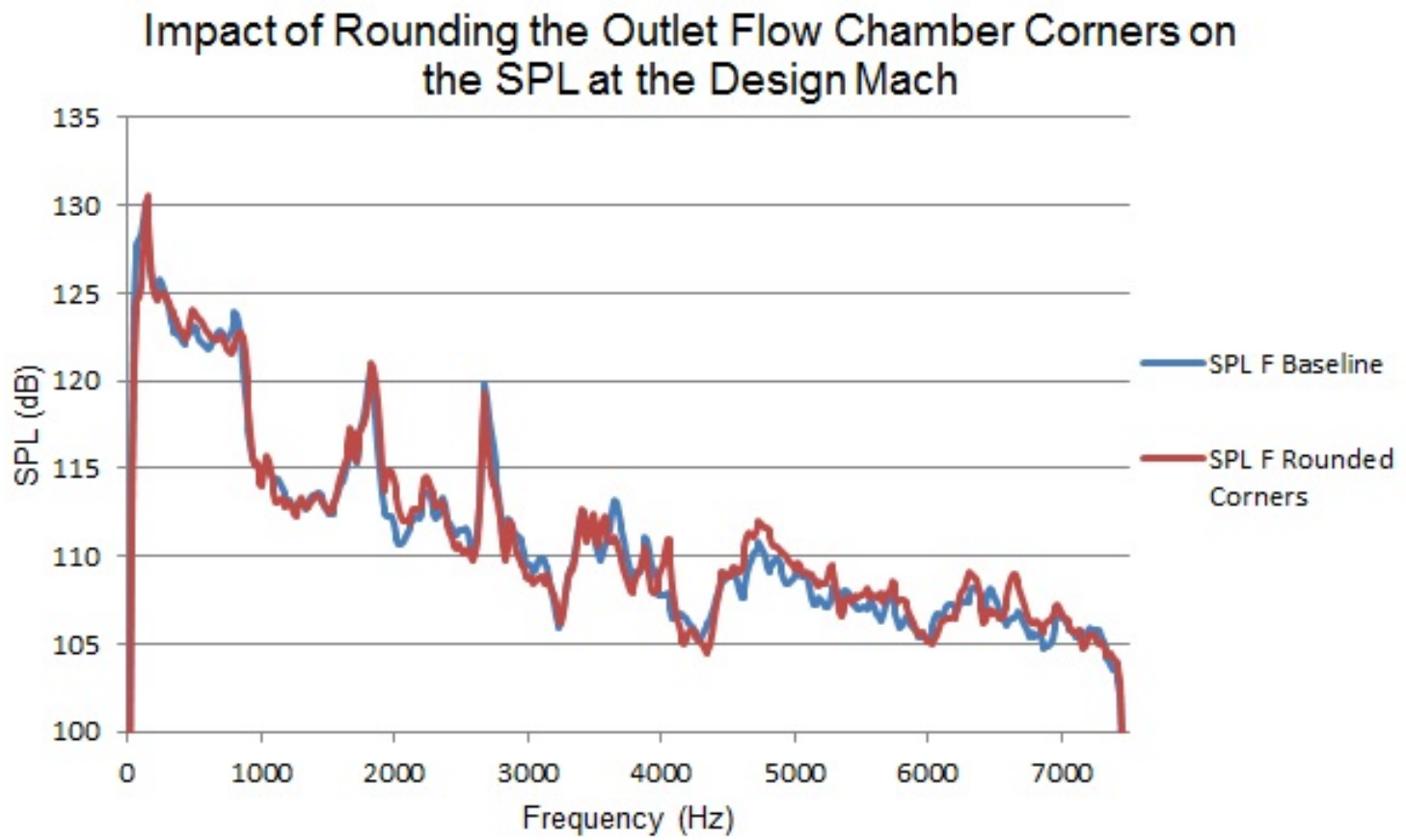

Figure 8.28: The SPL spectrum as measured at location F for the outlet flow chamber with rounded corners

placement used such that the tip protrudes above the layer of foam.

Although the thickness of the foam changes the area of the ejector (making it smaller thus causing larger flow velocities), the acoustic spectrum is astounding. As can be seen in figure 8.30 , the acoustic signature in the outlet flow chamber is completely changed. There are no peaks seen, no distinct valleys and a continuous decrease in SPL beginning at $100 \mathrm{~Hz}$. This test suggests that the noise recorded in previous tests truly stems from acoustic natural modes of the ejector. The entrainment ratio for this configuration becomes quite hard to estimate. The acoustic foam has an egg-crate type surface thus the area is decreased, however, the new effective area - thus entrained mass flow - is difficult to estimate.

The spectrum measured at location B is also quite interesting and is shown in 

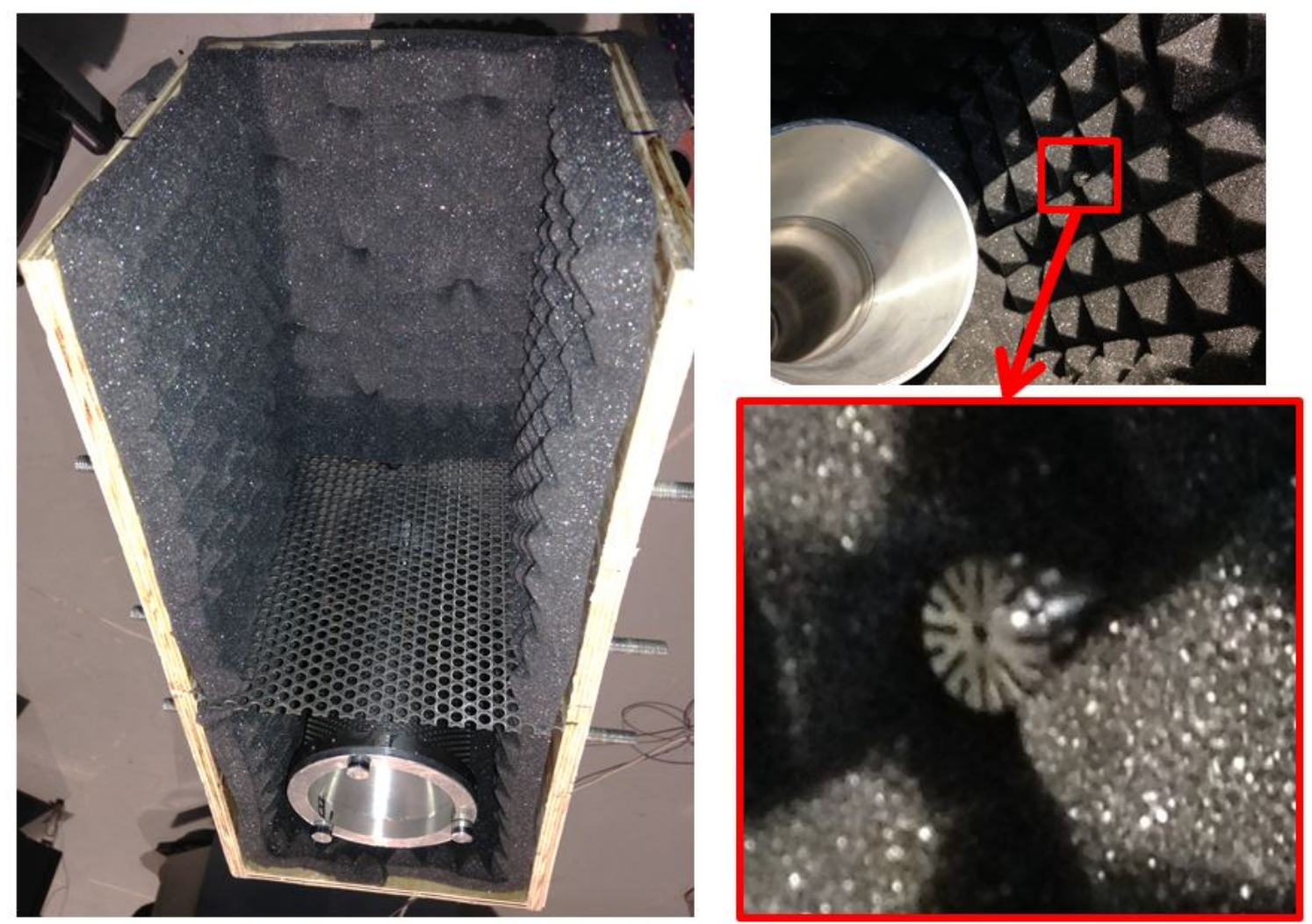

Figure 8.29: The outlet flow chamber lined with acoustic foam and a close up view of the microphone protruding beyond the foam

figure 8.31 .

Given that the inlet flow chamber is only partially lined with foam, the spectrum still exhibits some peaks, with the higher frequencies seemingly randomly attenuated. The natural transverse/azimuthal modes associated with the throat at $2800 \mathrm{~Hz}$ are completely gone. The author believes there are two possible explanations for this.

1. It is possible that the throat is still resonating but that the noise in the throat is not propagating to the microphone measurement location. This could be confirmed or negated by having a measurement in the throat

2. It may also be possible that the excitation of the throat is reliant on reflections from other portions of the scale model, such as the outlet flow chamber. Lining 


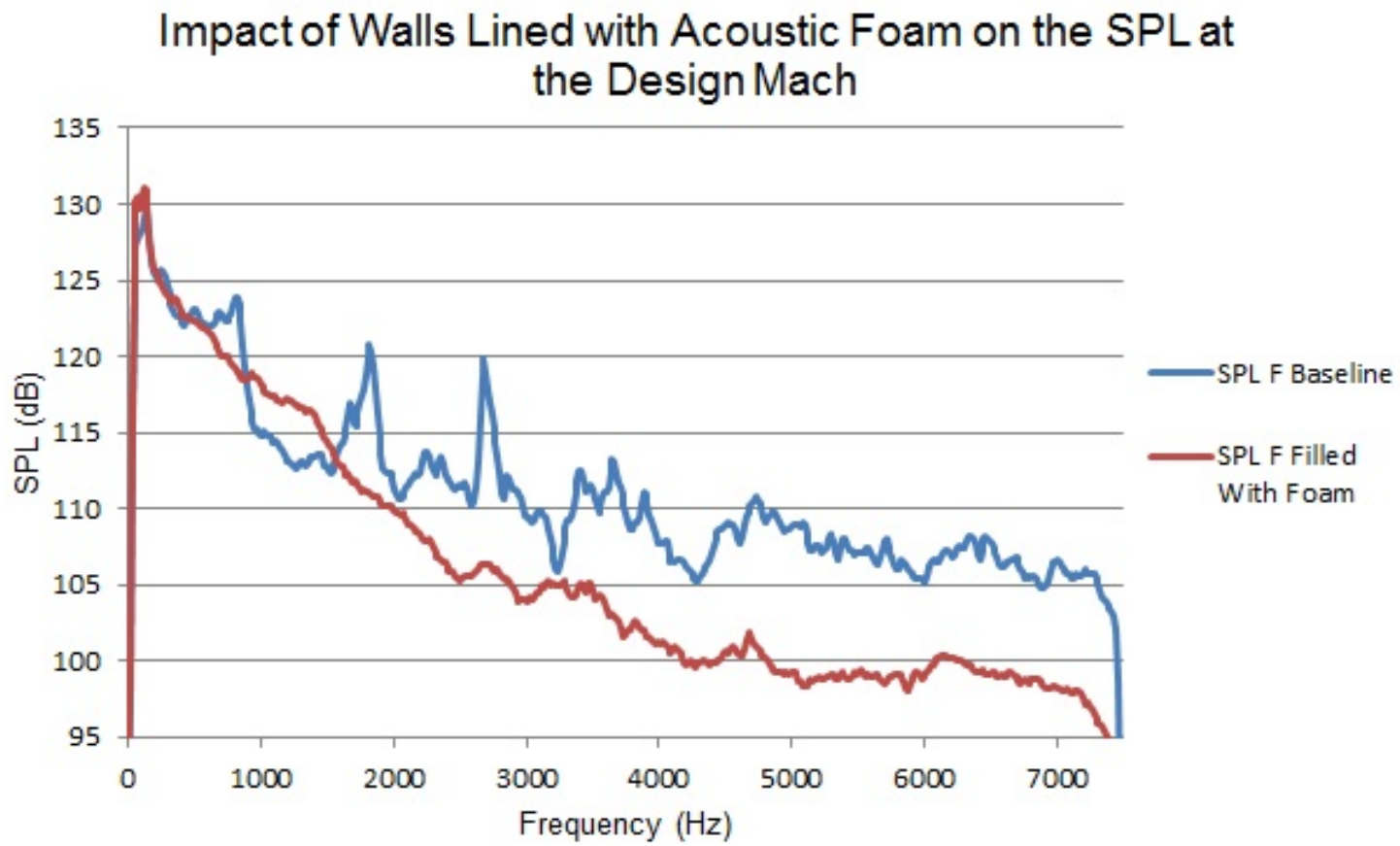

Figure 8.30: The SPL spectrum as measured at location F for the ejector walls lined with acoustic foam

the walls with foam would cause these reflections to be non-existent thus not allowing the throat modes to be excited

The natural longitudinal modes of the throat show no difference from the baseline measurements and this is also true of the entire ejector longitudinal mode. This is expected as the longitudinal modes do not depend on reflections at the walls. A damping mechanism placed in the path of these longitudinal modes would be required to eliminate them, however, this is difficult to achieve without affecting the flow.

The excitation of natural acoustic modes - especially those at frequencies with a high natural mode density - could be reduced by following certain guidelines. The field of room acoustics explain that having the most uniform distribution of natural acoustic modes is desirable. This could be achieved by carefully selecting the ejector geometry with each section in mind. The full significance of these results remains 


\section{Impact of Walls Lined with Acoustic Foam on the SPL at the Design Mach}

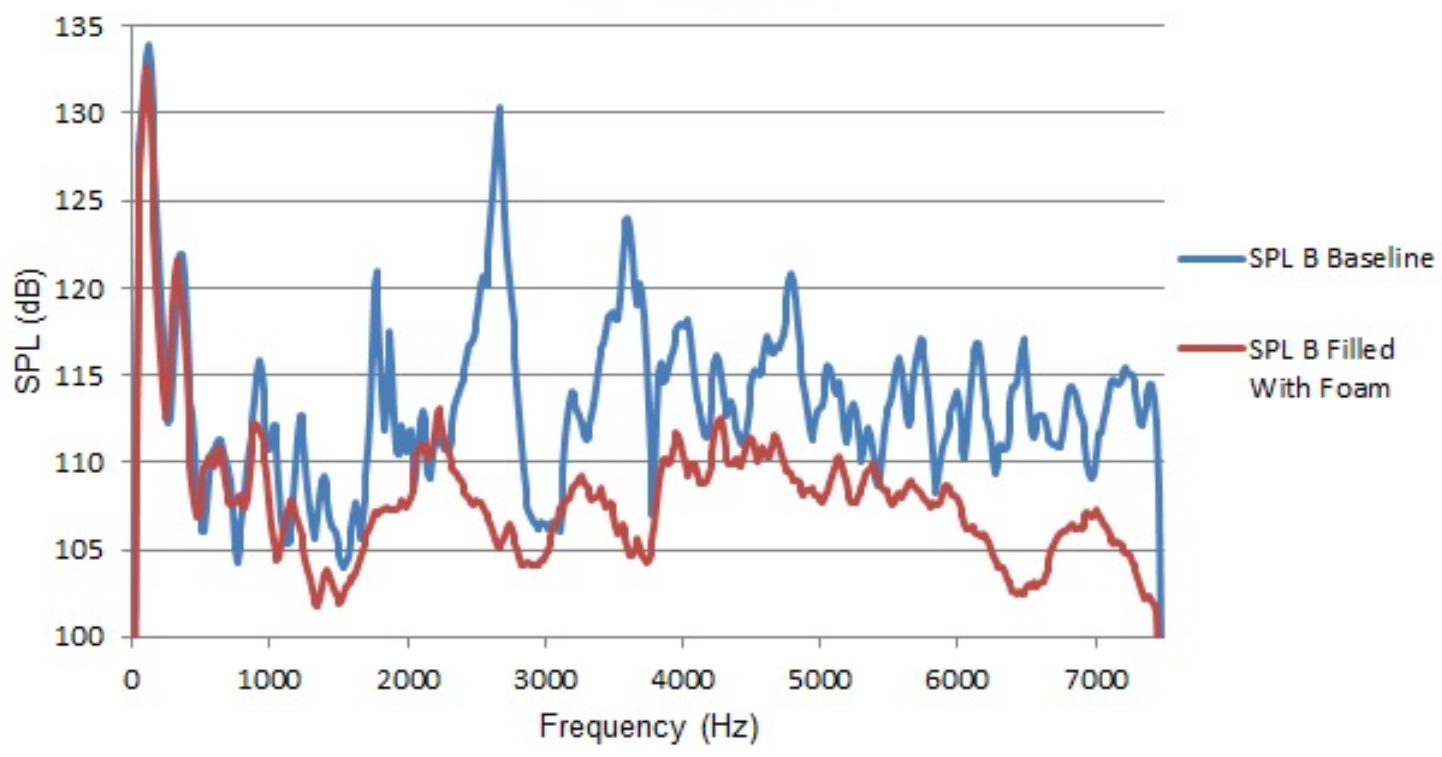

Figure 8.31: The SPL spectrum as measured at location B for the ejector walls lined with acoustic foam

unknown and would require further testing to be clarified. A few questions pertaining to the results at location $\mathrm{F}$ are particularly alluring: Why are the low frequency noise levels still so high? What is the source of the remaining noise, is it simple aerodynamic noise such as vortex sound? What factors dictate the slope of the curve? Further tests using a new building material which absorbs sound waves rather than reflects them could be used to evaluate the impact of new configurations on this noise generation mechanism. 


\section{Chapter 9}

\section{Contributions, Conclusions, Recommendations, Lessons Learnt and Future Work}

\subsection{Contributions}

After 800 wind tunnel runs, countless hours of data processing and too much coffee, a few noteworthy contributions can be emphasized. First and foremost, this work has shown than in the low frequency range, the acoustics of an ejector are dominated by natural modes of the different flow chambers. The intermittent tones can most likely be attributed to a resonance of the throat, more specifically, the coupling of some form of excitation from the jet with the throat first transverse mode of oscillation, as suggested in the literature.

A practical solution for decreasing the low frequency acoustics - that is a perforated cone placed at the mid-length of the perforated cylinder - has been found. This solution provides only a small decrease in entrainment ratio while effectively decreasing the amplitude of the noise from roughly the 0-3000 Hz range. This geometrically simple solution contains no moving parts and could easily (relatively so) 
be implemented in the full scale.

The design and manufacturing of a scale ejector has led to a highly modular platform from which numerous tests can be conducted. The fine tuning of the supply valve control system has also provided an adequate Mach number control not only for this work, but also for any other users of the facility wanting to employ low mass flow rates.

Finally, a number of configurations have been tested and eliminated as possibilities as noise decreasing techniques. Not only have many of the tested configurations proven that certain avenues of investigation are not worth pursuing, but these tests can be of great value to validate computational tests which might be performed at a later date. If a mechanism can be proposed for ejector acoustics, the tested configurations can serve to verify this mechanism under varying conditions.

\subsection{Conclusions}

The scale model ejector and silencer combination designed for this work allows for many different configurations to be tested. The noise levels generated by the ejector are found to be much greater than those generated outside the anechoic chamber thus noise contamination is unlikely. Future ejector geometries should be carefully designed such that there is no overlapping of natural modes, given that the largest peaks in the acoustic spectra seem to occur for frequency ranges exhibiting multiple acoustic modes.

Tests confirm that the measured spectrum is in fact dominated by natural modes and that these modes are quite difficult to supress. The shape, roughness and added surface features of the cone are found not to improve the ejector acoustics, sometimes making them worst. The use of a perforated cone placed in front of the solid cone 
proves to be the best method for reducing noise while keeping the entrainment ratio at an acceptable level. Many configurations, including perforated disks, screens and specially shaped cones have proven to be rather ineffective in lowering the acoustic levels or they incur too large of a decrease in entrainment ratio.

\subsection{Recommendations}

First and foremost, it is recommended that more emphasis be placed on the aerodynamics of the ejector, something which was not possible in this research given the lack of proper instruments. Analyzing the aerodynamic as well as acoustics of configuration changes would help in understanding the relationship between aerodynamics and acoustics. Performing tests of the baseline configuration with varying entrainment ratios (perhaps through selective blockage of the inlet perforated plate) would allow for a quantification of the change in acoustics due to a change in entrainment ratio.

In order to capture acoustic information in sensitive locations (such as the surface of the cone where conventional microphones would be destroyed) it is recommended to purchase fast response transducers such as Kulites. This would allow for a better understanding of the flow fluctuations.

It is recommended that a new module be purchased for the microphone data acquisition chassis which is capable of measuring multiple voltages as well as outputting a voltage/current signal. This would allow for tests to be conducted using a single code/computer making data synchronisation easier. Having more microphones (the microphone measurement system can readily accept 4 more microphones) would allow tests to be performed nearly twice as fast as microphone configuration changes would not be required. 
If a mass flow meter were installed somewhere in the air supply line, the measurement uncertainty could be greatly reduced as the entire calibration procedure of the primary nozzle would not be required. Alternatively, more accurate transducers would help reduced the uncertainty. A better valve or valve control algorithm may help reduce velocity fluctuations.

\subsection{Lessons Learnt}

If this project were to be repeated certain aspects would be undertaken differently.

Firstly, the scale model should be made from a minimum thickness of $3 / 4$ inch (19 $\mathrm{mm}$ ) plywood of the highest quality (unless an absorptive panel is opted for). All panels should be cut using a computer numerically controlled (CNC) router and made modular to allow for parametric geometry changes of any component. A method should also be devised to easily change the primary nozzle position and alignment. A quick and easy way to hold the microphones in place, such as some type of clamping mechanism or cam lock, should be investigated.

\subsection{Future Work}

Like any good scientific investigation, there are more questions now than when the research was begun. There remain many points which would require further investigation. These points are phrased as the following questions:

What role does the perforated cylinder play on the acoustics? More specifically, how does the OAR affect the acoustic performance of an ejector? For a given perforated cylinder OAR, what effect does the hole size have? Are micro perforations a viable option? What is the velocity profile at the outlet of the perforated cylinder? 
Would a pitot-traverse a short distance from the perforated cylinder provide useful information? Would a varying OAR be beneficial?

How well is the diffuser performing? Is there any separation occurring in the diffuser? Would a new diffuser configuration be beneficial to the acoustics? Why do silencer configurations which protrude into the diffuser tend to reduce the entire ejector longitudinal mode? Could these protrusions be replaced by artificial protrusions in the form of air microjet injection?

What impact does the throat length, diameter and length to diameter ratio have on acoustics? Can the natural modes of the throat be easily attenuated using conventional methods? Would a lobbed mixer placed at the throat inlet or outlet improve the ejector acoustics or make them worst?

Could the ejector chambers and throat be partitioned in such a way to shift the acoustic modes to higher frequencies which can more easily be attenuated using passive methods? Is active control of the acoustic modes a feasible option?

Only through investigation and experimentation can these questions be answered, and finding these answers is likely to open a pandoras box of questions. 


\section{Bibliography}

[1] M. El Hayek and A. Hammoud, "Prediction of liquid jet pump performance using computational fluid dynamics," in Proceedings of the $4^{\text {th }}$ WSEAS International Conference on Fluid Mechanics and Aerodynamics, pp. 148-153, August 2006.

[2] J. Kracik, V. Dvorak, and J. Kolar, "Development of air to air ejector for supersonic wind tunnel," EPJ Web of Conferences, vol. 67, pp. 02059-p.1 02059-p. 4, 2014.

[3] Y. J. Lee, S. H. Kang, S. S. Yang, and S. J. Kwon, "Starting characteristics of the hypersonic wind tunnel with the mach number variation," Journal of Mechanical Science and Technology, vol. 28, pp. 2197-2204, 2014.

[4] J. Wilson, A. Sgondea, D. E. Paxson, and B. N. Rosenthal, "Parametric investigation of thrust augmentation by ejectors on a pulsed detonation tube," Journal of Propulsion and Power, vol. 23, pp. 108-115, 2007.

[5] V. Baev, D. Y. Moskvichev, and A. Potapkin, "Control of thrust characteristics of a scramjet combustor with vibrational combustion using acoustic resonators," Combustion, Explosion and Shock Waves, vol. 36, pp. 553-556, 2000. 
[6] L. Bernal and V. Sarohia, "Entrainment and mixing in thrust augmenting ejectors," Tech. Rep. AFOSR-TR-82-1008, Jet Propulsion Laboratory, February 1982.

[7] W. J. Presz, G. Reynolds, and C. Hunter, "Thrust augmentation with mixer/ejector systems," in $40^{\text {th }}$ AIAA Aerospace Sciences Meeting and Exhibit, pp. 1-10, January 2002.

[8] G. Singhal, Mainuddin, R. Tyagi, A. Dawar, and P. Subbarao, "Pressure recovery studies on a supersonic coil with central ejector configuration," Optics and Laser Technology, vol. 42, pp. 1145-1153, 2010.

[9] J. W. Molloy, "Design and testing of scaled ejector-diffusers for jet engine test facility applications," Master's thesis, Naval Postgraduate School, Monterey, California, 1983.

[10] G. Saunders, "A3 subscale diffuser test article design," in $45^{\text {th }}$ AIAA/ASME/SAE/ASEE Joint Propulsion Conference and Exhibit, pp. 1-10, August 2009.

[11] S. Mitra, N. C. Daltrophe, and J. Gilron, "A novel eductor-based mbr for the treatment of domestic wastewater," Water Research, vol. 100, pp. 65-79, 2016.

[12] J.-H. Lee, A. Sameen, V. Sanal Kumar, and H. Kim, "Studies on ejector systems for hydrogen fuel cell," in $41^{\text {st }}$ AIAA/ASME/SAE/ASEE Joint Propulsion Conference and Exhibit, pp. 1-8, July 2005.

[13] F. Marsano, L. Magistri, and A. Massardo, "Ejector performance influence on a solid oxide fuel cell anodic recirculation system," Journal of Power Sources, vol. 129, pp. 216-228, 2004. 
[14] S. Elbel and N. Lawrence, "Review of recent developments in advanced ejector technology," International Journal of Refrigeration, vol. 62, pp. 1-18, 2015.

[15] Chem Process Systems, "Steam jet ejectors." www.chemprosys.com/products/ vacuum-ejectors/steam-jet-ejectors. Accessed: 21/11/2017.

[16] Fox Venturi Products, "Fox mini-eductors." www.foxvalve.com/ mini-eductors/introduction. Accessed: 21/11/2017.

[17] T. Sriveerakul, S. Aphornratana, and K. Chunnanond, "Performance prediction of steam ejector using computational fluid dynamics: Part 1. validation of the cfd results," The International Journal of Thermal Sciences, vol. 46, pp. 812$822,2007$.

[18] S. Karthick, M. R. Srisha, and G. Jagadeesh, "Parametric experimental studies on mixing characteristics within a low area ratio rectangular supersonic gaseous ejector," Physics of Fluids, vol. 28, pp. 076101-1 - 076101-26, 2016.

[19] D. Middleton, "The noise of ejectors," tech. rep., Ministry of Aviation, October 1963. Reports and Memoranda No. 3389.

[20] A. Krothapalli and Y. Hsia, "Discrete tones generated by a supersonic jet ejector," The Journal of the Acoustical Society of America, vol. 99, pp. 777-784, September 1995.

[21] K. Ahuja, K. Massey, and A. Entrekin, "Contribution of mixing within an ejector to the farfield noise measurements," in AIAA $34^{\text {th }}$ Aerospace Sciences Meeting and Exhibit, pp. 1-28, January 1996.

[22] G. Leventhall, "A review of published research on low frequency noise and its effects," tech. rep., Defra, May 2003. 
[23] I. Idelchik, Handbook of Hydraulic Resistance. Begell House, 3 ed., 1996.

[24] J. F. Wilby, "Noise of steam-injection water heaters (abstract only)," The Journal of the Acoustical Society of America, vol. 58, no. S16, 1975.

[25] A. Siami, M. Asayesh, A. Najafi, and A. Hamedanian, "Sound noise control of a steam ejector in a typical power plant: Design, manufacturing, and testing a silencer-muffler (abstract only)," World Academy of Science, Engineering and Technology, vol. 10, no. 6, 2016.

[26] B. Haynes and J. Dickens, "Attenuation of noise from steam injection in poultry scald tanks," tech. rep., U.S. Department of Agriculture, October 1976. no. S153.

[27] B. Quinn, "Effect of aeroacoustic interactions on ejector performance," Journal of Aircraft, vol. 12, no. 11, pp. 914-916, 1975.

[28] P. Bradshaw, J. Flintoff, and D. Middleton, "Unexplained scale effects in ejector shroud "howling"," Journal of Sound and Vibration, vol. 7, no. 2, pp. 183-190, 1968.

[29] B. Quinn, "Interactions between screech tones and ejector performance," Journal of Aircraft, vol. 14, pp. 467-473, May 1977.

[30] J. Campbell, K. Korkan, and H. Viets, "Investigation of acoustic interactions in jet thrust augmenting ejectors," tech. rep., Naval Air Systems Command, Department of the Navy, March 1981. AD A106083.

[31] A. M. Abdel-Fattah and S. C. Favaloro, "Duct resonance and its effect on the performance of high-pressure ratio axisymmetric ejectors," AIAA Journal, vol. 26, pp. 791-798, July 1988. 
[32] K. Zaman, M. Clem, and A. Fagan, "Noise from a jet discharged into a duct and its suppression," International Journal of Aeroacoustics, vol. 12, pp. 189-214, February 2013.

[33] M. Dahl and O. McDaniel, "The performance of jet noise suppression devices for industrial applications," Journal of Vibration, Acoustics, Stress and Reliability in Design, vol. 107, pp. 303-309, July 1985.

[34] K. Zaman, M. Clem, A. Fagan, and The United States of America as represented by the Administrator of the National Aeronautics and Space Administration, "Us. patent application for a "system and method for suppression of unwanted noise in ground test facilities"." Washington, DC, USA, Filed 26 April 2013. US Patent No. 8,939,253 B1.

[35] S. J. Krasheninnikov, A. Mironov, E. Paulukov, V. Zitenev, J. Julliard, and E. Maingre, "An experimental study of 2-d mixer/ejector noise and thrust characteristics," in AIAA and CEAS Aeroacoustics Conference, $2^{\text {nd }}$, pp. 1-14, May 1996.

[36] G. Raman and R. Taghavi, "Aeroacoustic characteristics of a rectangular multielement supersonic jet mixer-ejector nozzle," The Journal of Sound and Vibration, vol. 207, pp. 227-248, April 1997.

[37] V. Kousnetsov, A. Sobolev, and E. Maslova, "Efficiency of jet noise ejector suppressor with sound absorbing treatments," in $4^{\text {th }}$ AIAA/CEAS Aeroacoustics Conference, pp. 721-730, June 1998.

[38] T. Oishi and J. Strange, "Nozzle/ejector noise source characteristics," in rth $^{2}$ AIAA/CEAS Aeroacoustics Conference, pp. 1-9, May 2001. 
[39] T. Z. Dong and R. R. Mankbadi, "Large-eddy simulations of sound due to turbulence mixing inside an ejector," in $2^{\text {nd }} A I A A$ and CEAS Aeroacoustics Conference, pp. 1-12, May 1996.

[40] A. Samanta and J. Freund, "Finite-wavelength scattering of incident vorticity and acoustic waves at a shrouded-jet exit," Journal of Fluid Mechanics, vol. 612, pp. 407-438, October 2008.

[41] J. Freund, V. Topalian, A. Samanta, and J. Kim, "Super-resonances in aedc altitude test cells," in 2007 U.S. Air Force TEE Days, (Destin, FL, USA), pp. 131-137, February 2007.

[42] V. Topalian and J. Freund, "Acoustic resonance in a model ducted-jet system," AIAA Journal, vol. 48, pp. 1348-1360, July 2010.

[43] R. Freuler and K. Montgomery, "Reducing large pressure fluctuations in an engine test cell by modifying the exhaust blast basket end configuration," in First Joint CEAS/AIAA Aeroacoustics Conference (16 $6^{\text {th }}$ AIAA Aeroacoustics Conference), (Munich, Germany), pp. 903-909, June 1995.

[44] N. L. Helgeson, W. Cheeseman, S. Fann, and J. Scott, "Reduction of noise from the j-52 and f-404 jet engines during static testing using the noise attenuation device (nad)," tech. rep., Naval Facilities Engineering Command, June 2009. TR-2319-ENV.

[45] M. Kandula and B. Vu, "On the scaling laws for jet noise in subsonic and supersonic flow," NASA KSC-2003-040, pp. 1-18, May 2003.

[46] C. K. W. Tam, K. Viswanathan, K. Ahuja, and J. Panda, "The sources of jet noise: Experimental evidence," The Journal of Fluid Mechanics, vol. 615, pp. 253-292, November 2008. 
[47] D. A. Bies and C. H. Hansen, Engineering Noise Control, Theory and Practice. Spon Press, 3 ed., 2003.

[48] A. Marsh, "Noise measurements around a subsonic air jet impinging on a plane, rigid surface," The Journal of the Acoustical Society of America, vol. 33, pp. 1065-1066, August 1961.

[49] B. Henderson, "The connection between sound production and jet structure of the supersonic impinging jet," The Journal of the Acoustical Society of America, vol. 111, pp. 735-747, February 2002.

[50] F. Alvi, H. Lou, C. Shih, and R. Kumar, "Experimental study of physical mechanisms in the control of supersonic impinging jets using microjets," The Journal of Fluid Mechanics, vol. 613, pp. 55-83, October 2008.

[51] C.-M. Ho and N. N.S., "Dynamics of an impinging jet. part 1. the feedback phenomenon," The Journal of Fluid Mechanics, vol. 105, pp. 119-142, July 1980.

[52] T. Davis, A. Edstrand, F. Alvi, L. Cattafesta, D. Yorita, and K. Asai, "Investigation of impinging jet resonant modes using unsteady pressure-sensitive paint measurements," Experiments in Fluids, vol. 56, pp. 1-13, May 2015.

[53] P. Panickar and G. Raman, "Criteria for the existence of helical instabilities in subsonic impinging jets," Physics of Fluids, vol. 19, pp. 106103-1-106103-17, May 2007.

[54] B. Vinoth and E. Rathakrishnan, "Effect of impinging plate geometry on the self-excitation of subsonic impinging jets," Journal of Fluids and Structures, vol. 27, pp. 1238-1251, November 2011. 
[55] C. Tam and K. Ahuja, "Theoretical model of discrete tone generation by impinging jets," Journal of Fluid Mechanics, vol. 214, pp. 67-87, May 1990.

[56] G. Neuwerth, "Acoustic feedback of a subsonic and supersonic free jet which impinges on an obstacle," tech. rep., NASA, 1974.

[57] R. Mehta and P. J.K., "Flow structure of a supersonic jet impinging on an axisymmetric deflector," Indian Journal of Engineering 85 Materials Sciences, vol. 4, pp. 178-188, October 1997.

[58] R. Mehta, "Numerical simulation of supersonic turbulent jets impinging on an axisymmetric deflector," Indian Journal of Engineering $\&$ Materials Sciences, vol. 9, pp. 227-236, August 2002.

[59] J.-H. Park, T.-S. Lee, and Y.-G. Kim, "A study of supersonic jets impinging on an axisymmetric cone (article in korean)," Journal of the Korean Society for Aeronautical 6 Space Sciences, vol. 31, pp. 26-31, September 2003.

[60] R. Seryu and J. Iwamoto, "Impingement of underexpanded jet on cone," in $10^{\text {th }}$ International Conference on Fluid Control, Measurements, and Visualization, (Moscow, Russia), 2009.

[61] M. Rennie, "Effect of jet length on pressure fluctuations in 3/4 open-jet wind tunnels," January 2000.

[62] G. Paal and I. Vaik, "Unsteady phenomena in the edge tone," International Journal of Heat and Fluid Flow, vol. 28, pp. 575-586, April 2007.

[63] M. Ibrahim, "Experimental and theoretical investigations of edge tones in high speed jets," Journal of Fluid Science and Technology, vol. 8, no. 1, pp. 1-19, 2013. 
[64] J. Lepicovsky and K. Ahuja, "Experimental results on edge-tone oscillations in high-speed subsonic jets," AIAA Journal, vol. 23, pp. 1463-1468, October 1985.

[65] A. Krothapalli, K. Karamcheti, Y. Hsia, and D. Baganoff, "Edge tones in highspeed flows and their application to multiple-jet mixing," AIAA Journal, vol. 21, pp. 937-938, July 1983.

[66] I. Vaik, R. Varga, and G. Paal, "Frequency and phase characteristics of the edge tone, part i," Periodica Polytechnica Mechanical Engineering, vol. 58, pp. 55-67, April 2014.

[67] C. Segoufin, B. Fabre, and L. De-lacombe, "Experimental investigation of the flue channel geometry influence on edge-tone oscillations," Acustica/Acta Acustica, vol. 90, pp. 966-975, September/October 2004.

[68] R. Azad, "Turbulent flow in a conical diffuser: A review," Experimental Thermal and Fluid Science, vol. 13, pp. 318-337, November 1996.

[69] K. Zaman and M. Dahl, "Some observations on transitory stall in conical diffusers," in 28 th Aerospace Sciences Meeting, (Reno, NV, USA), January 1990.

[70] R. Blevins, Applied Fluid Dynamics Handbook. Van Nostrand Reinhold Co., 1984.

[71] J. Willis, "Spurious pressure fluctuations in wind tunnels," The Journal of The Acoustical Society of America, vol. 43, pp. 1049-1054, May 1968.

[72] A. Kwong and A. Dowling, "Unsteady flow in diffusers," The Journal of Fluids Engineering, vol. 116, pp. 842-847, December 1994. 
[73] K. Fehse and W. Neise, "Low frequency sound generated by flow separation in a conical diffuser," European Journal of Mechanics - B/Fluids, vol. 19, pp. 637652, December 2000.

[74] L. Van Lier, S. Dequand, and A. Hirschberg, "Aeroacoustics of diffusers: An experimental study of typical industrial diffusers at reynolds numbers of $O$ $\left(10^{5}\right), "$ Journal of the Acoustical Society of America, vol. 109, pp. 108-115, September 2001.

[75] A. Martin and F. Thiele, "Investigation of flow separation and transition to turbulence in an axisymmetric diffuser," in 42 $42^{\text {nd }}$ AIAA Aerospace Sciences Meeting and Exhibit, (Reno, NV, USA), January 2004.

[76] L. Hoefener, W. Nitsche, A. Carnarius, F. Thiele, H. (ed) Rath, C. (ed) Holze, H. (ed) Heinemann, R. (ed) Henke, and (ed) Honlinger H., Experimental and Numerical Investigations of Flow Separation and Transition to Turbulence in an Axisymmetric Diffuser. In: New Results in Numerical and Experimental Fluid Mechanics V. Notes on Numerical Fluid Mechanics and Multidisciplinary Design (NNFM), vol. 92. Springer, 2004.

[77] G. Scavone, An Acoustic Analysis of Single-Reed Woodwind Instruments With an Emphasis on Design and Performance Issues and Digital Waveguide Modeling Techniques. PhD thesis, Department of Music, Stanford University, 1997.

[78] H. Kuttruff, Room Acoustics. Spon Press, 5 ed., 2009.

[79] A. Miklos, P. Hess, and Z. Bozoki, "Application of acoustic resonators in photoacoustic trace gas analysis and metrology," Review of Scientific Instruments, vol. 72, pp. 1937-1955, April 2001. 
[80] M. R. Schroeder, "The "schroeder frequency" revisited," The Journal of the Acoustical Society of America, vol. 99, pp. 3240-3241, May 1996.

[81] W. C. Sabine, Collected Papers on Acoustics. Cambridge Harvard University Press, 1 ed., 1922.

[82] F. Giardino, "The design, fabrication, and commissioning of a high-speed aeroacoustic wind tunnel for studies of surface pressure fluctuations beneath turbulent boundary layers," Master's thesis, Ottawa-Carleton Institute for Mechanical and Aerospace Engineering. Carleton University, 2018.

[83] M. Jeffries, The Commissioning and Enhancement of a Blow Down Type Wind Tunnel. PhD thesis, Ottawa-Carleton Institute for Mechanical and Aerospace Engineering. Carleton University, 1994.

[84] M. D. Kibsey, "Influence of mach number on profile loss of axial-flow turbine blades," Master's thesis, Ottawa-Carleton Institute for Mechanical and Aerospace Engineering. Carleton University, 2015.

[85] S. R. Hall, "Investigation of the effects of compressibility on profile pressure loses in axil turbine cascades," Master's thesis, Ottawa-Carleton Institute for Mechanical and Aerospace Engineering. Carleton University, 2011.

[86] G. Abramovich, The Theory of Turbulent Jets. MIT Press, 1 ed., 1963.

[87] J. W. Gauntner, J. N. Livingood, and P. Hrycak, "Survey of literature on flow characteristics of a single turbulent jet impinging on a flat plate," NASA TN D-5652, pp. 1-22, February 1970.

[88] Brel \& Kjr, Product Data, 1/4" DeltaTron Pressure-field Microphones - Types 4944- $A$ and 4944-B, 2009. 
[89] S. Kline and F. McClintock, "Describing uncertainties in single-sample experiments," Mechanical Engineering, vol. 75, pp. 3-8, 1953.

[90] S. Tavoularis, Measurement in Fluid Mechanics. Cambridge University Press, 1 ed., 2005.

[91] J. Van Blitterswyk, "Experimental characterization of turbulent motions using wall-pressure measurements in low reynolds number turbulent boundary layers," Master's thesis, Ottawa-Carleton Institute for Mechanical and Aerospace Engineering. Carleton University, 2016.

[92] M. Kandula, "On the scaling laws and similarity spectra for jet noise in subsonic and supersonic flow," NASA KSC-2008-053, pp. 1-50, March 2008.

[93] M. Lighthill, "On sound generated aerodynamically i. general theory," Proceedings of the Royal society, vol. 211, pp. 564-587, March 1952.

[94] S. Kannappan, Introduction to Pipe Stress Analysis. John Wiley \& Sons, 1 ed., 1986.

[95] Y. Z. Erdil, J. Zhang, and C. A. Eckelman, "Holding strength of screws in plywood and oriented strandboard," Forest Products Journal, pp. 55-62, June 2002.

[96] M. Vorlander, Auralization Fundamentals of Acoustics, Modelling, Simulation, Algorithms and Acoustic Virtual Reality. Springer, 1 ed., 2008.

[97] P. Dutta, K. S. Sumit, N. Nityananda, and P. Nairit, "Numerical study on flow separation in $90^{\circ}$ pipe bend under high reynold number by k- $\epsilon$ modelling," Engineering Science and Technology, an International Journal, vol. 19, pp. 904910, 2016. 
[98] K. W. Kinzie, B. S. Henderson, and H. H. Haskin, "Aeroacoustic characteristics of model jet test facility flow conditioners," Institute of Physics Publishing, vol. 13, pp. 1608-1614, 2002.

[99] M. J. Doty and H. H. Haskin, "Investigation of flow conditioners for compact jet engine simulator rig noise reduction," in $1^{\text {th }}$ AIAA/CEAS Aeroacoustics Conference (32 ${ }^{\text {nd }}$ AIAA Aeroacoustics Conference), pp. 1-17, June 2011.

[100] F. Moloney, C. Wickramaratne, E. Almatrafi, D. Goswami, E. Stefanakos, and R. Guldiken, "Flow conditioning techniques for a bent pipe in a constrained latent heat storage system," in IMECE2016-65730, vol. 19, pp. 1-8, November 2016 .

[101] R. Mehta and P. Bradshaw, "Design rules for small low speed wind tunnels," The Aeronautical Journal of the Royal Aeronautical Society, pp. 443-449, 1979.

[102] O. Turgut and M. Sari, "Experimental and numerical study of turbulent flow and heat transfer inside hexagonal duct," Heat and Mass Transfer, vol. 49, pp. 543-554, April 2013.

[103] R. R. Burley and H. D. E., "Experimental evaluation of honeycomb/screen configurations and short contractions section for nasa lewis research center's altitude wind tunnel," NASA Technical Paper 2692, pp. 1-27, May 1987.

[104] V. Kulkarni, N. Sahoo, and S. D. Chavan, "Simulation of honeycomb-screen combinations for turbulence management in a subsonic wind tunnel," Journal of Wind Engineering and Industrial Aerodynamics, vol. 99, pp. 37-45, January 2011.

[105] Y. Bayazit, E. M. Sparrow, and D. D. Joseph, "Perforated plates for fluid 
management: Plate geometry effects and flow regimes," International Journal of Thermal Sciences, vol. 85, pp. 104-111, 2014.

[106] E. P. Spearman, S. J. A., and M. J. Reader-Harris, "Comparison of velocity and turbulence profiles downstream of perforated plate flow conditioners," Flow Measurement and Instrumentation, vol. 7, no. 3/4, pp. 181-199, 1996.

[107] S. Chue, "Pressure probes for fluid measurement," Progress in Aerospace Sciences, vol. 16, no. 2, pp. 147-223, 1975.

[108] C. Editors: Tropea, A. L. Yarin, and J. F. Foss, Springer Handbook of Experimental Fluid Mechanics. Springer, 1 ed., 2007.

[109] B. J. Mckeon and A. J. Smits, "Static pressure correction in high reynolds number fully developed turbulent pipe flow," Institute of Physics Publishing, vol. 13, pp. 1608-1614, 2002.

[110] A. Hussain and M. Hasan, "The 'whistler-nozzle' phenomenon," Journal of Fluid Mechanics, vol. 134, pp. 431-458, September 1983. 


\section{Appendix A}

\section{Calibration Curves}

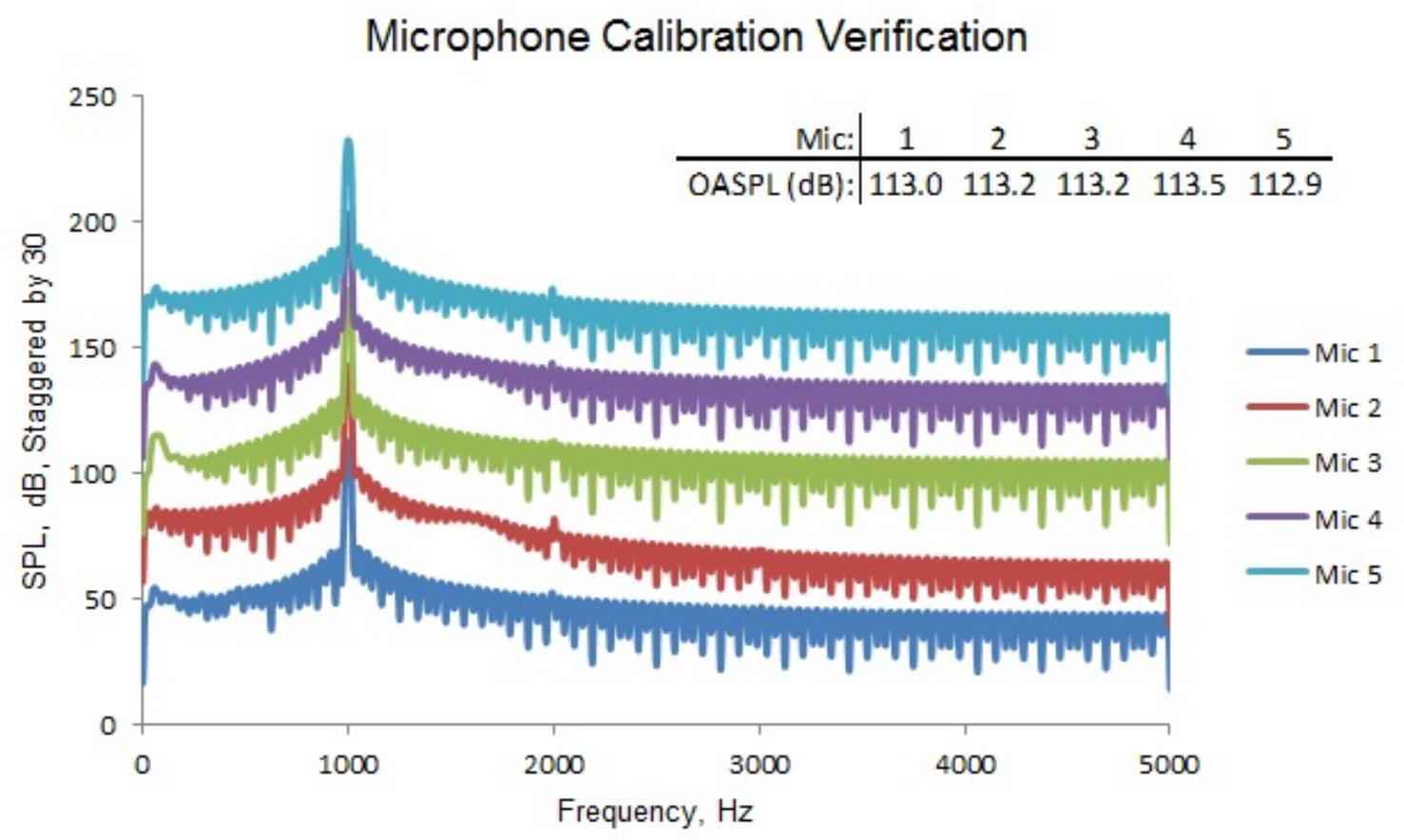

Figure A.1: The microphone calibration results 


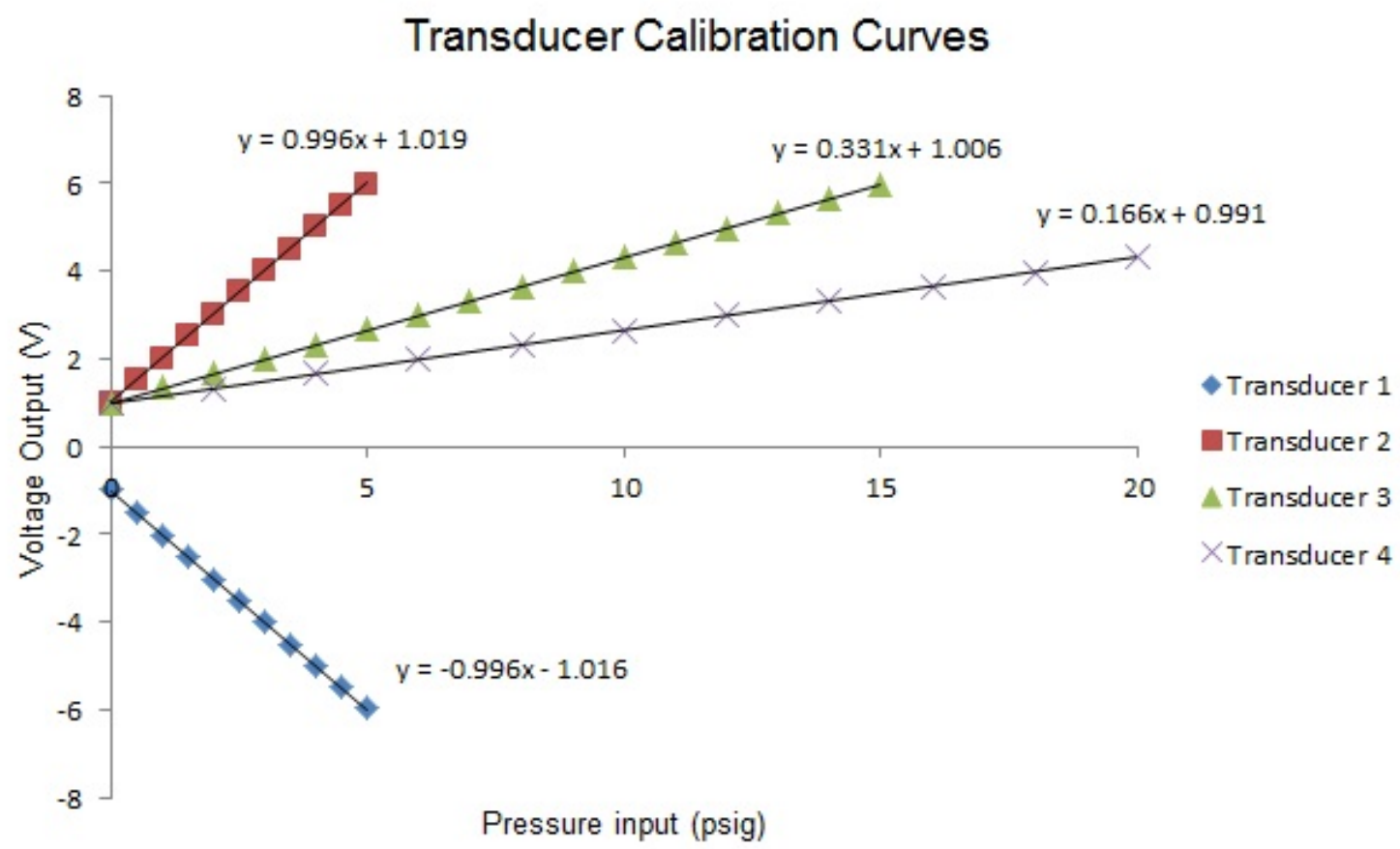

Figure A.2: The differential pressure transducer calibration results

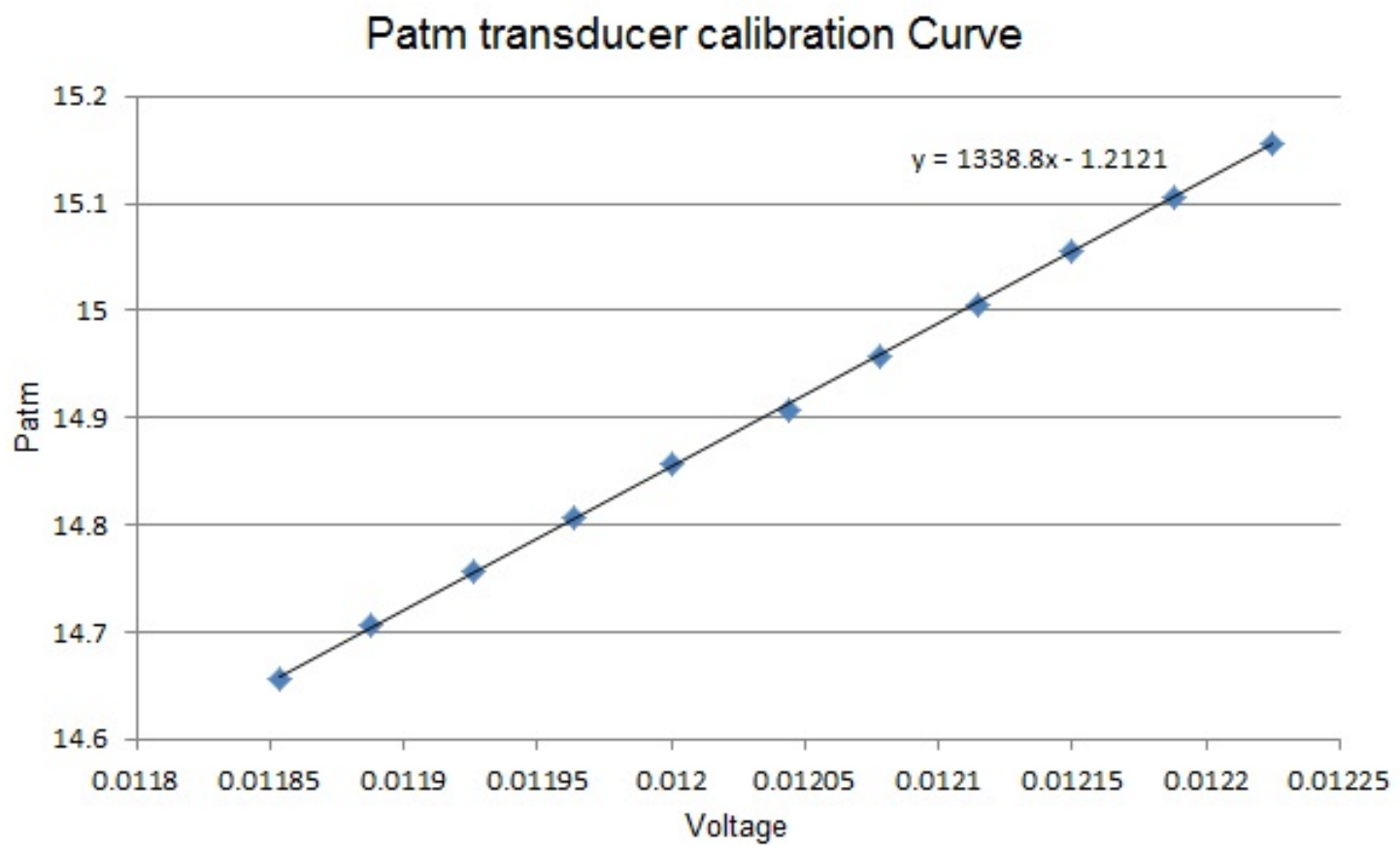

Figure A.3: The atmospheric pressure transducer calibration results, data from Giardino 82 


\section{Appendix B}

\section{Roots of the Bessel Function Derivative}

Table B.1: Roots of the Bessel Function Derivative

\begin{tabular}{rlllllll}
\multicolumn{7}{c}{$\sigma_{j k}$} \\
& \multicolumn{7}{c}{$\mathrm{k}$} \\
& & 0 & 1 & 2 & 3 & 4 & 5 \\
\hline & 0 & 1.8412 & 3.0542 & 4.2012 & 5.3175 & 6.4156 \\
& 1 & 3.8317 & 5.3314 & 6.7061 & 8.0152 & 9.2824 & 10.5199 \\
$\mathrm{j}$ & 2 & 7.0156 & 8.5363 & 9.9695 & 11.3459 & 12.6819 & 13.9872 \\
& 3 & 10.1735 & 11.7060 & 13.1704 & 14.5858 & 15.9641 & 17.3128 \\
4 & 13.3237 & 14.8636 & 16.3475 & 17.7887 & 19.1960 & 20.5755
\end{tabular}




\begin{abstract}
Appendix C
Noise Measured at the Valve for All Mach

Numbers
\end{abstract}




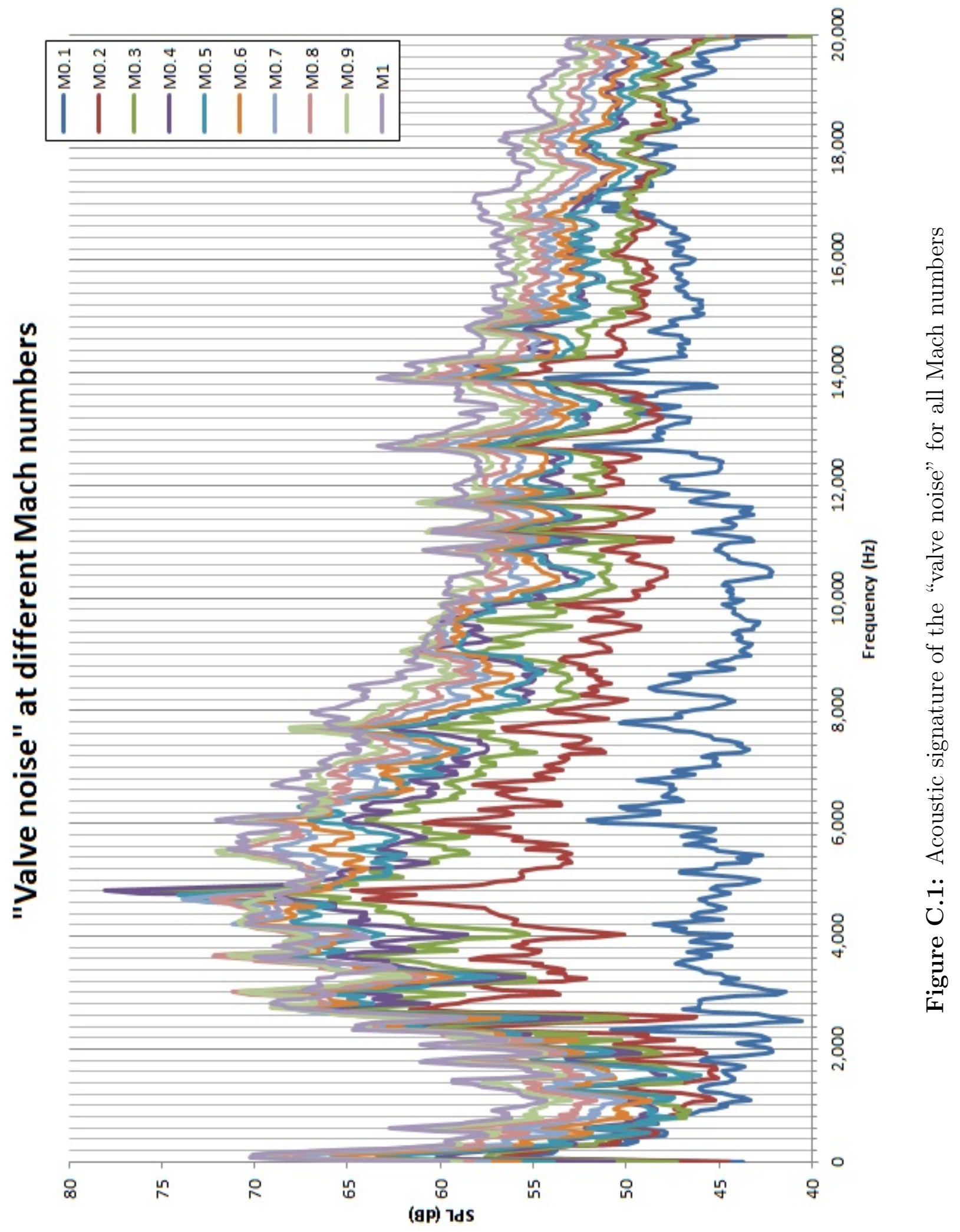

